\title{
Assessment of Feasibility of the Beneficial Use of Waste Heat from the Advanced Test Reactor
}

July 2012

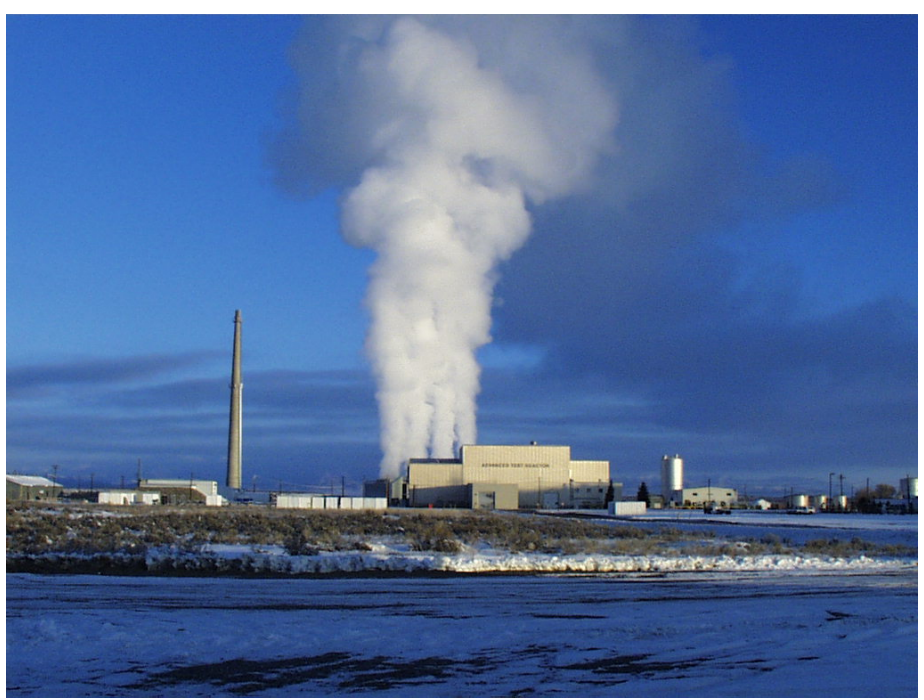

The INL is a U.S. Department of Energy National Laboratory operated by Battelle Energy Alliance

Idaho National Laboratory 


\section{DISCLAIMER}

This information was prepared as an account of work sponsored by an agency of the U.S. Government. Neither the U.S. Government nor any agency thereof, nor any of their employees, makes any warranty, expressed or implied, or assumes any legal liability or responsibility for the accuracy, completeness, or usefulness, of any information, apparatus, product, or process disclosed, or represents that its use would not infringe privately owned rights. References herein to any specific commercial product, process, or service by trade name, trade mark, manufacturer, or otherwise, does not necessarily constitute or imply its endorsement, recommendation, or favoring by the U.S. Government or any agency thereof. The views and opinions of authors expressed herein do not necessarily state or reflect those of the U.S. Government or any agency thereof. 


\section{Assessment of Feasibility of the Beneficial Use of Waste Heat from the Advanced Test Reactor}

July 2012

Idaho National Laboratory Idaho Falls, Idaho 83415

http://www.inl.gov

Prepared for the

U.S. Department of Energy

Office of Nuclear Energy

Under DOE Idaho Operations Office

Contract DE-AC07-05ID14517 



\section{Assessment of Feasibility of the Beneficial Use of Waste Heat from the Advanced Test Reactor}

INL/EXT-12-25004

July 2012

Prepared by:

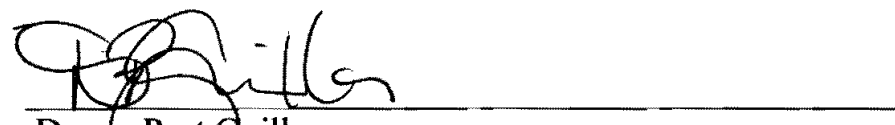

Donta Post Quillen

Research Engineer, Advanced Process and Decision

Systems Dept.
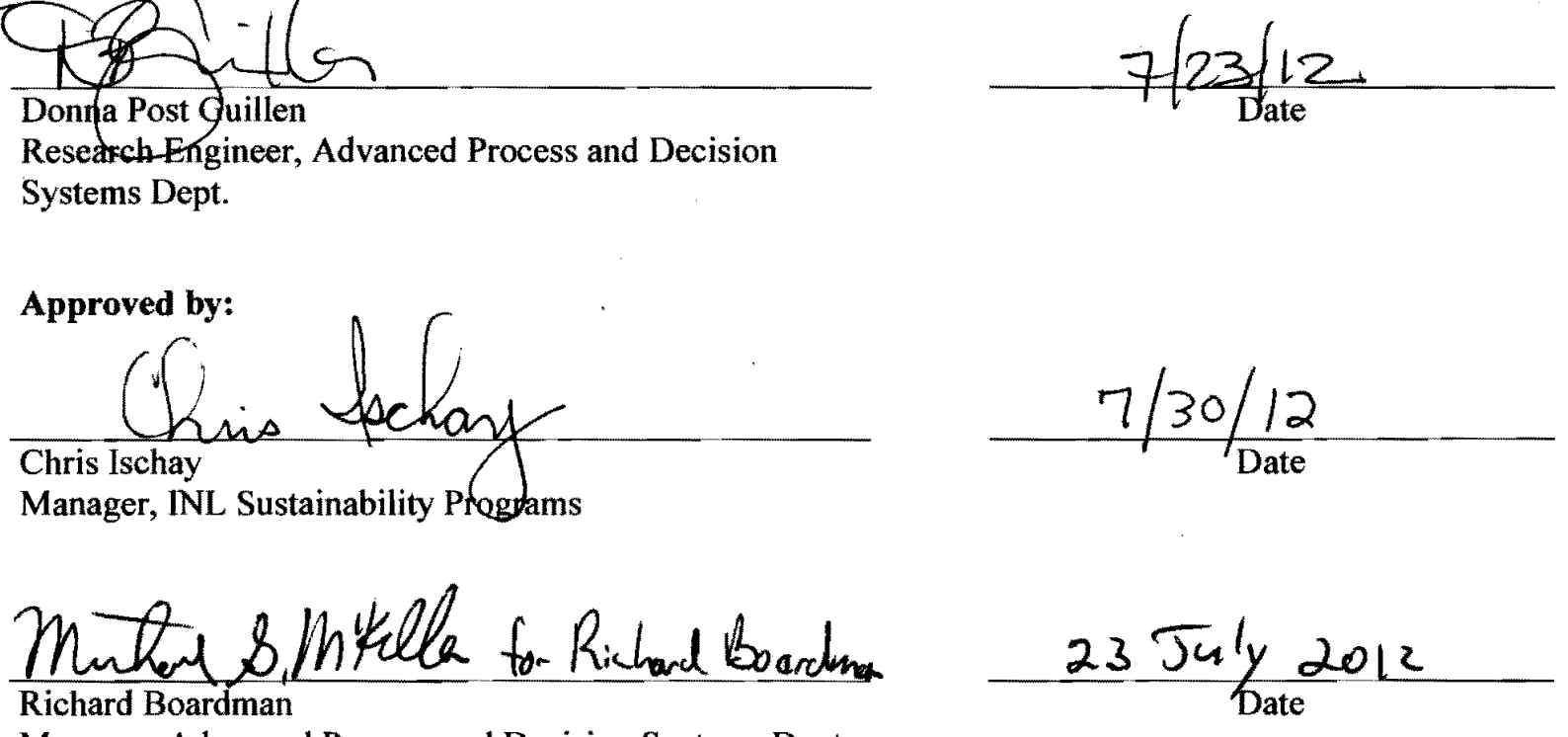

Manager, Advanced Process and Decision Systems Dept. 



\begin{abstract}
This report investigates the feasibility of using waste heat from the Advanced Test Reactor secondary coolant system to preheat air for space heating of TRA-670. The existing, but currently nonfunctioning waste heat recovery system was assessed for technical and economic feasibility. Hot water would be extracted from the secondary coolant system loop and pumped to a new plate and frame heat exchanger from which heat would be transferred to the tertiary glycol loop for preheating of outdoor air in the heating and ventilation system. Data from Advanced Test Reactor operations over the past 10 years indicates that heat from the reactor coolant was available (when needed for heating) for $43.5 \%$ of the year on average. The potential energy cost savings from using waste heat to preheat intake air would be $\$ 285 \mathrm{~K} / \mathrm{yr}$. The total project cost is estimated to be $\$ 9.68 \mathrm{M}$, which includes operating and maintenance costs for the first 5 years. Technical, safety, and logistics considerations of the glycol waste heat recovery system are outlined. Other opportunities for using waste heat and reducing water usage at the Advanced Test Reactor are also discussed.
\end{abstract}




\section{CONTENTS}

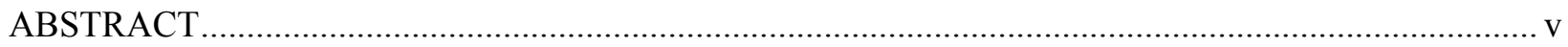

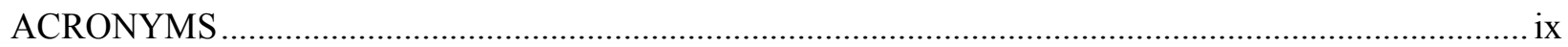

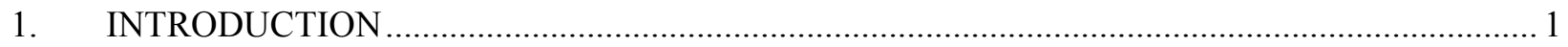

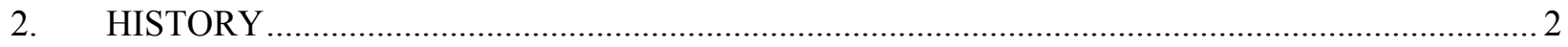

3. COMMENTS ON PROCESS FLOW DIAGRAM …............................................................... 6

3.1 New g-WHRS System .......................................................................................... 7

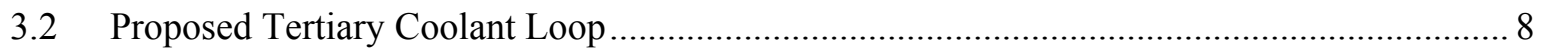

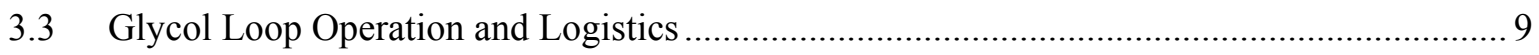

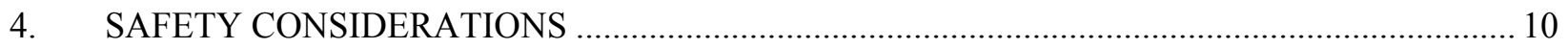

5. USE OF WASTE HEAT FOR SPACE HEATING ................................................................... 11

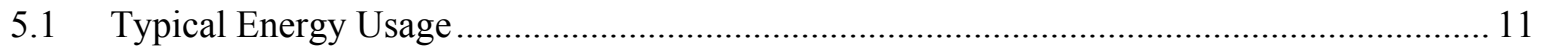

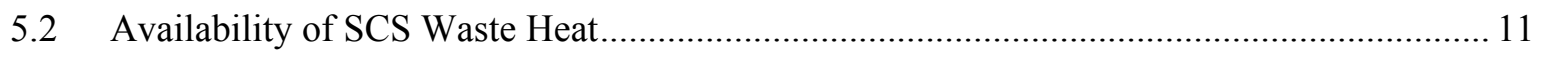

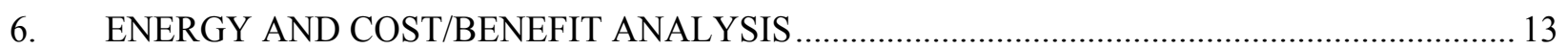

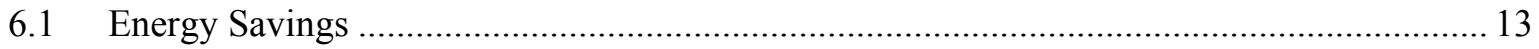

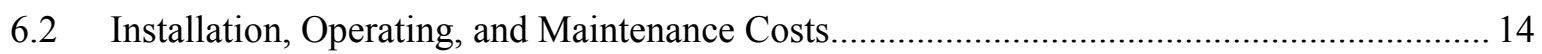

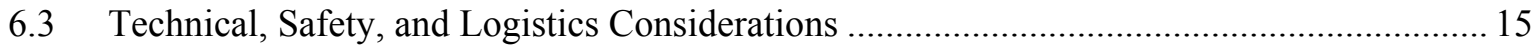

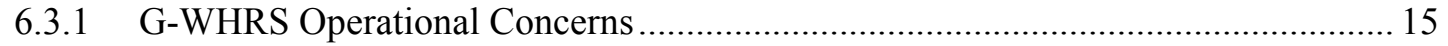

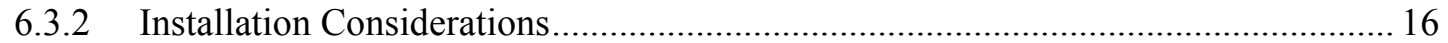

6.3.3 Safety Reviews and Operating Procedures ......................................................... 17

7. OTHER ENERGY AND WATER EFFICIENCY OPPORTUNITIES AT ATR .......................... 18

7.1 Alternate Waste Heat Recovery Concepts ........................................................................ 18

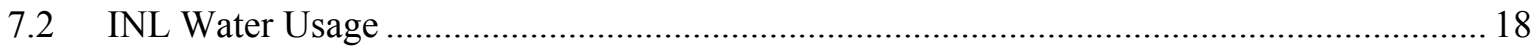

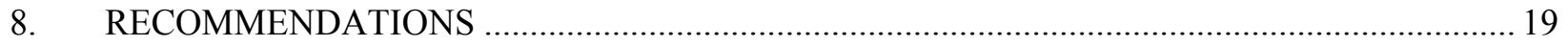

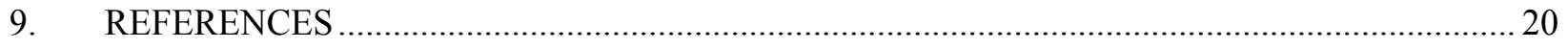

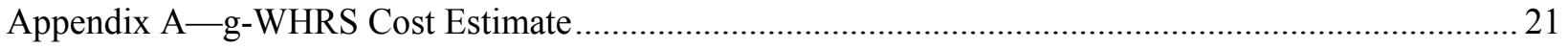

\section{FIGURES}

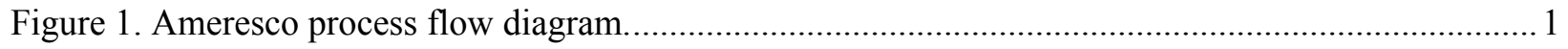

Figure 2. Old WHRS abandoned piping and pipe supports in canal area..............................................

Figure 3. Old WHRS abandoned piping outside of HVS-4 filter access compartment. ............................. 3

Figure 4. Old WHRS piping to the downstream side of the face and bypass damper in HVS-1................ 3

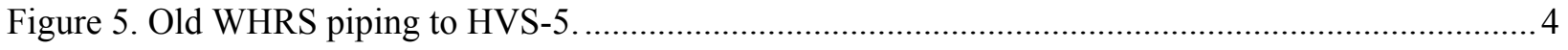


Figure 6. Old WHRS piping in TRA-670 layup area..

Figure 7. Old WHRS pump house (TRA-676, presently the fitness facility) on north side of

TRA-670.

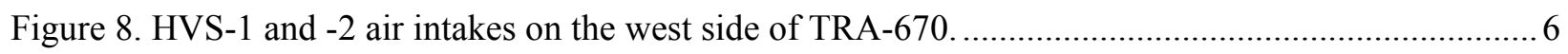

Figure 9. HVS-3, -4 , and -5 air intakes on the east side of TRA-670 ................................................... 6

Figure 10. ATR SCS header pit located on north side of TRA-670 ...................................................... 7

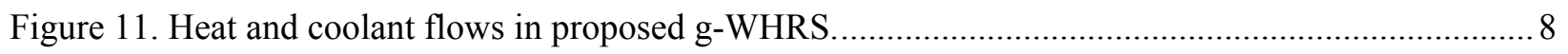

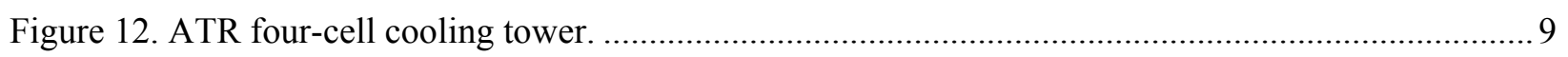

Figure 13. Commercial building energy usage splits (U.S. DOE, 2010) ............................................ 11

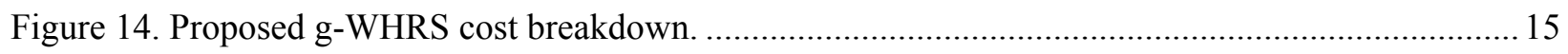

\section{TABLES}

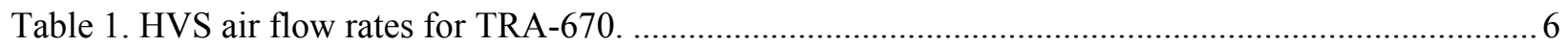

Table 2. ATR full-power hours over the past 10 years (source: Marjorie Owens, ATR)......................... 12

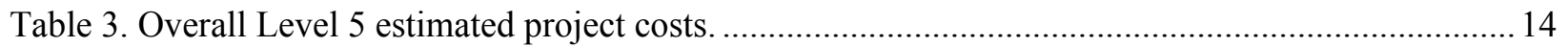

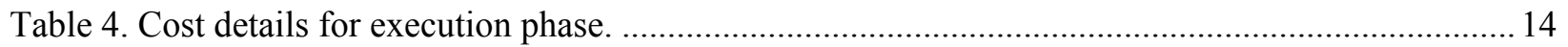




\section{ACRONYMS}

$\begin{array}{ll}\text { ATR } & \text { Advanced Test Reactor } \\ \text { DG } & \text { Diesel Engine Generator } \\ \text { DOE } & \text { Department of Energy } \\ \text { ECM } & \text { Energy Conservation Measure } \\ \text { GHG } & \text { Greenhouse Gas } \\ \text { g-WHRS } & \text { Glycol Waste Heat Recovery System } \\ \text { HVS } & \text { Heating and Ventilation System } \\ \text { INL } & \text { Idaho National Laboratory } \\ \text { PCS } & \text { Primary Coolant System } \\ \text { PFD } & \text { Process Flow Diagram } \\ \text { PRA } & \text { Probabilistic Risk Assessment } \\ \text { SCS } & \text { Secondary Coolant System } \\ \text { UFSAR } & \text { Upgraded Final Safety Analysis } \\ \text { USQ } & \text { Unreviewed Safety Questions } \\ \text { WHRS } & \text { Waste Heat Recovery System }\end{array}$




\section{Assessment of Feasibility of the Beneficial Use of Waste Heat from the Advanced Test Reactor}

\section{INTRODUCTION}

This report assesses the feasibility of using waste heat from the Advanced Test Reactor (ATR) at Idaho National Laboratory (INL) to preheat air for space heating of TRA-670. It evaluates a concept proposed by Ameresco, and discusses other potential energy and water efficiency opportunities at ATR.

The installation of a glycol waste heat recovery system (g-WHRS) to preheat outside air for building TRA-670, located at the ATR Complex, was proposed. The Ameresco provided process flow diagram (PFD), shown in Figure 1, was accompanied by the following paragraph:

"This ECM involves recovery of heat from the ATR cooling tower cooling water loop for use in preheating outdoor ventilation air for TRA-670. The combined outdoor intake air for four HVUs, including HVS-1, $-2,-3$, and -4 , totals 114,300 cfm according to design airflow schematics. The outdoor intake air in each of these HVUs is currently preheated by electric resistance coils. Implementation of this ECM would include installation of a heat exchanger to transfer heat from the $120^{\circ} \mathrm{F}$ cooling water to a new glycol loop. The heated glycol would then preheat outdoor air through a heating coil on the intake of each of the four HVUs. This ECM would result in substantial electric savings by reducing the heating load on the existing electric resistance preheat coils. The attached schematic shows a basic arrangement of the proposed heat recovery system."

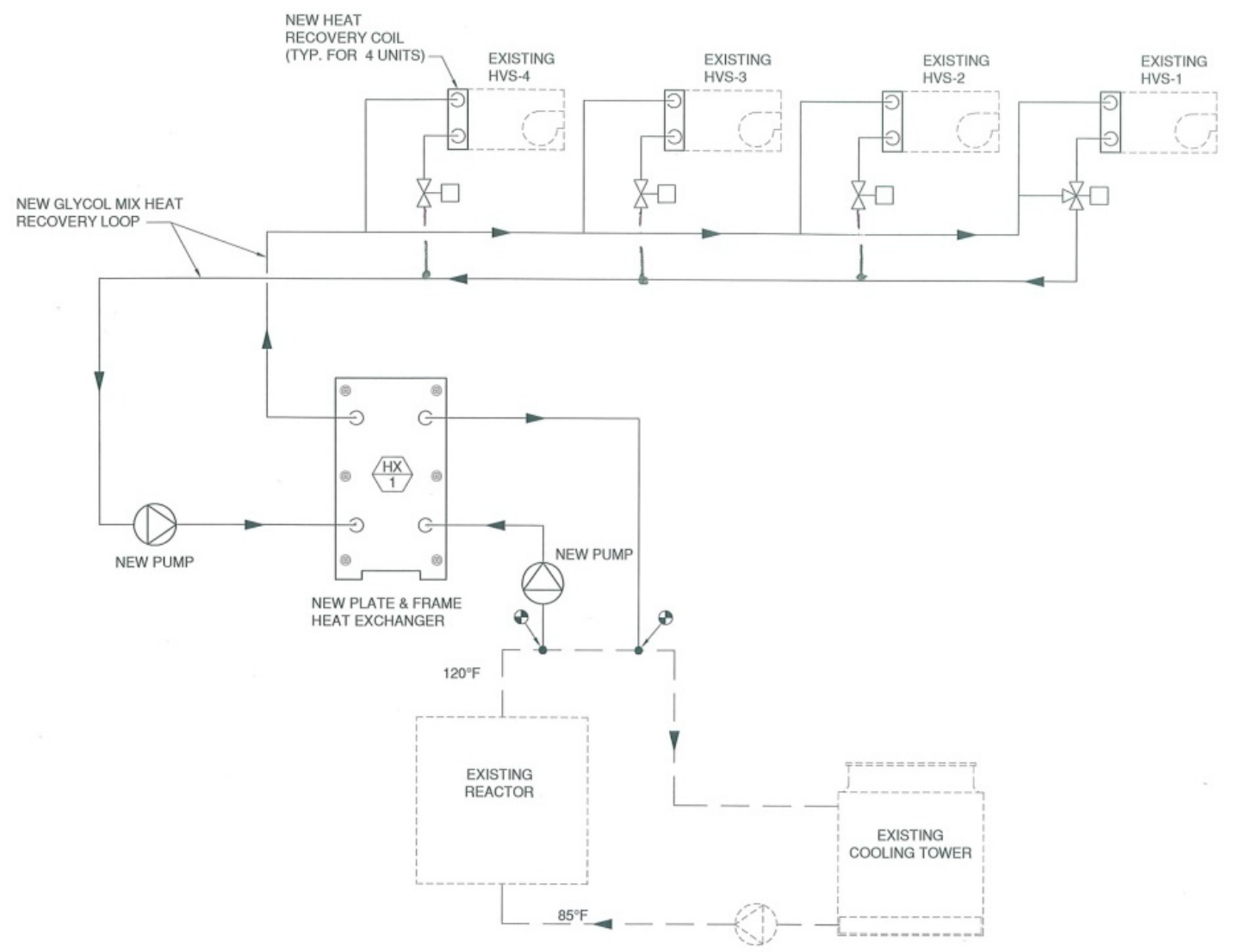

Figure 1. Ameresco process flow diagram. 


\section{HISTORY}

Constructed in 1967, the ATR is the second-oldest of three reactors still in operation at INL. A waste heat recovery system (WHRS) constructed at ATR during the late 1980s operated only briefly. The original motivation for the WHRS was the replacement of the deteriorating central steam plant with electrical heating. The existing WHRS was constructed in two phases. The first phase of the project involved design and installation of electrical resistance heating for all of the heated buildings at the ATR Complex. The second phase included design and construction of the WHRS loop.

The ATR WHRS was a piping/mechanical system that circulated warm water (at approximately $130^{\circ} \mathrm{F}$ ) from the ATR secondary coolant system (SCS) through heating coils that were part of the heating and ventilation systems (HVS) for 14 buildings at the ATR Complex in a district heating type arrangement. The WHRS underwent shakedown testing during FY 1990 and began operation during the FY 1991 heating season. Freeze up of the finned tube hot water coils during a commercial power outage resulted in flooding of the basement in TRA-604 (Kinnaman 1993). The system was placed in a dry layup configuration, which was later changed to "abandoned in place" (SES-2002-457, Rev. 1; Drawing 171227, 171211, 171200, etc.).

Figure 2 shows the old WHRS piping located in the canal area. The piping is situated along the walls high above the floor level. The white insulation covering the piping is marked with yellow and black labels to indicate that it is part of the WHRS. Figure 3 shows a placard with "Waste Heat Recovery System Abandoned in Place" near the old WHRS piping to HVS-4. Similar placards have been placed on the abandoned WHRS piping throughout the facility. WHRS piping to the HVS is shown in Figures 4 and 5. Figure 6 shows the old WHRS piping in the TRA-670 layup area. The building (TRA-676) on the north side of TRA-670 that formerly housed the pumps for the old WHRS has been reconfigured into a fitness facility (Figure 7).

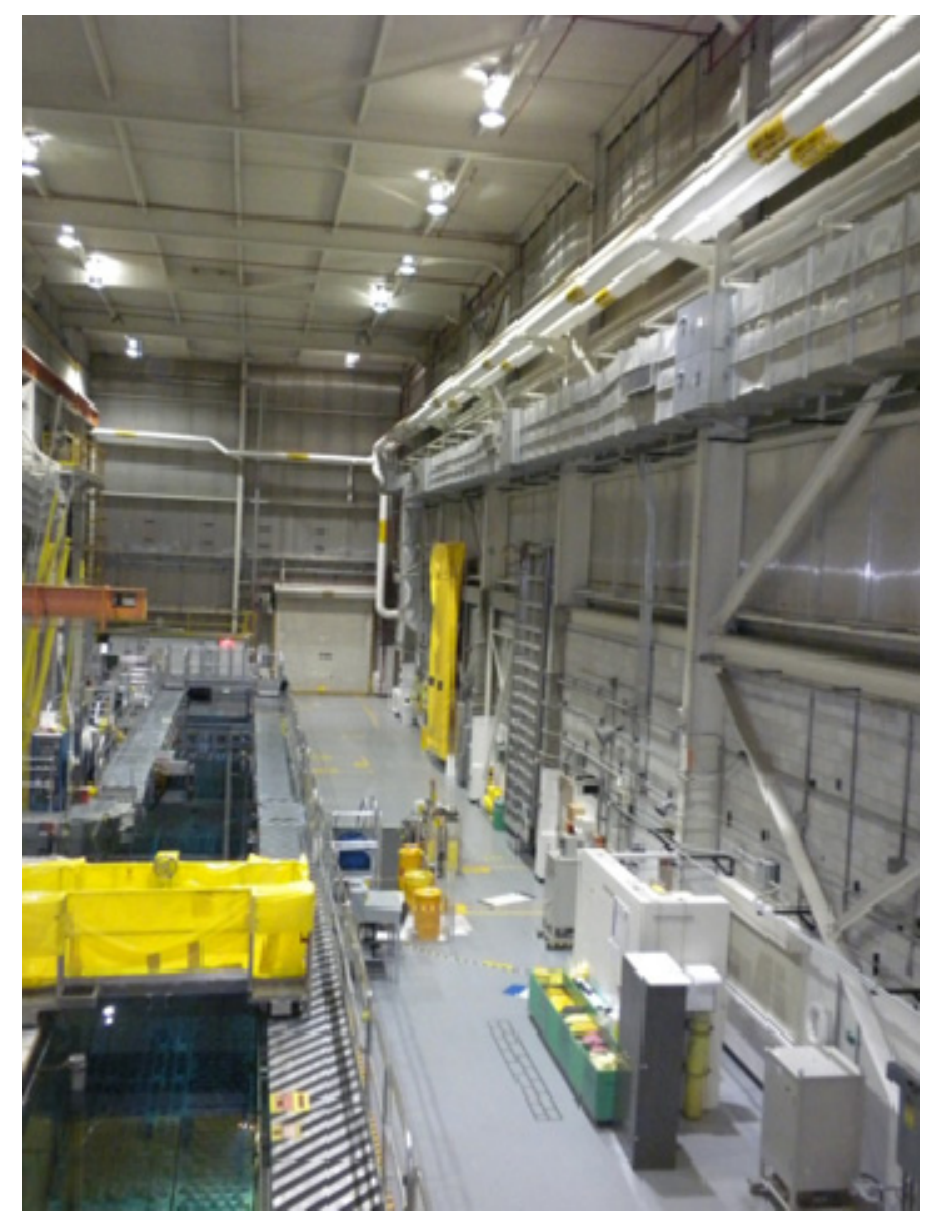

Figure 2. Old WHRS abandoned piping and pipe supports in canal area. 


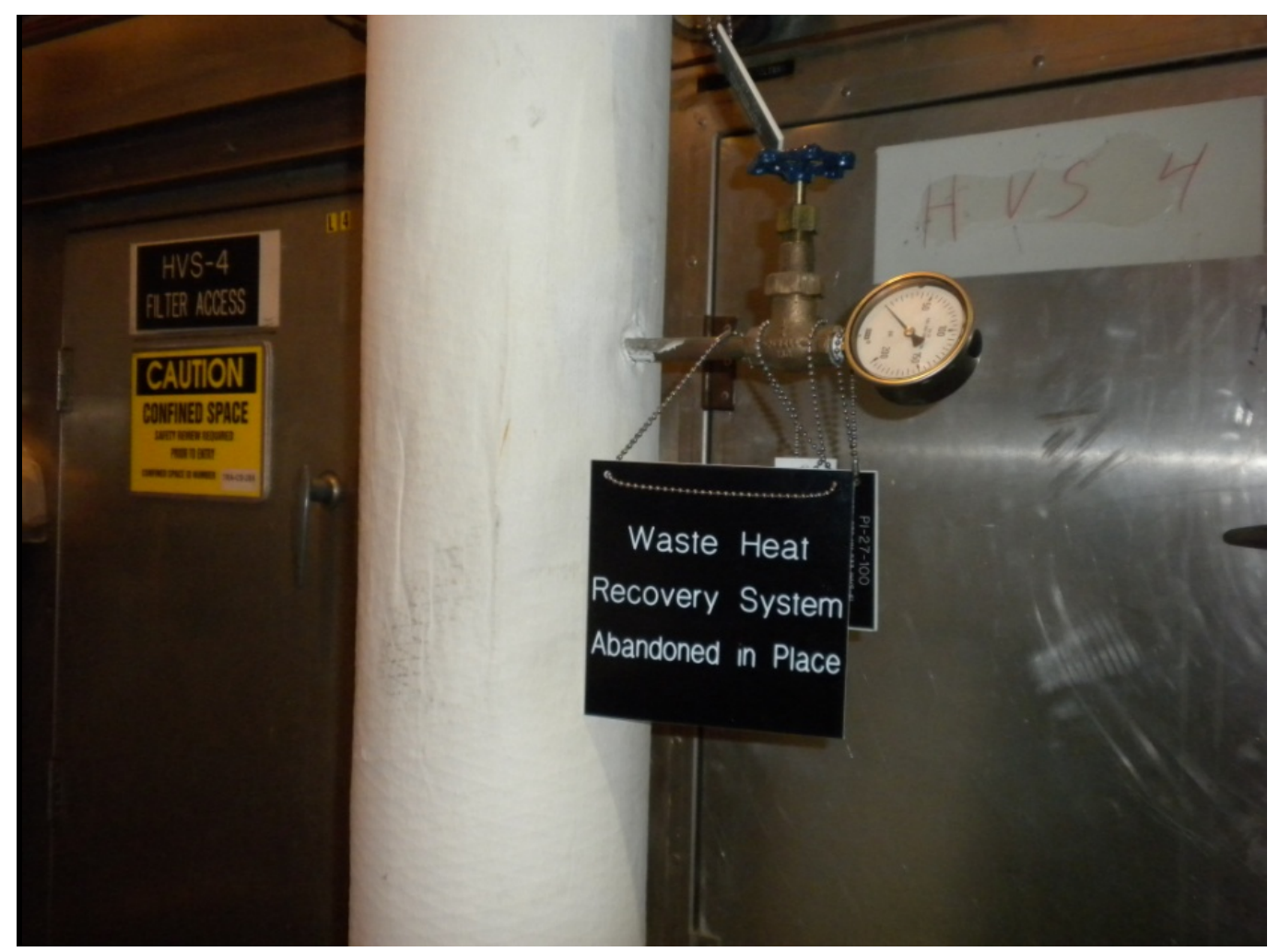

Figure 3. Old WHRS abandoned piping outside of HVS-4 filter access compartment.

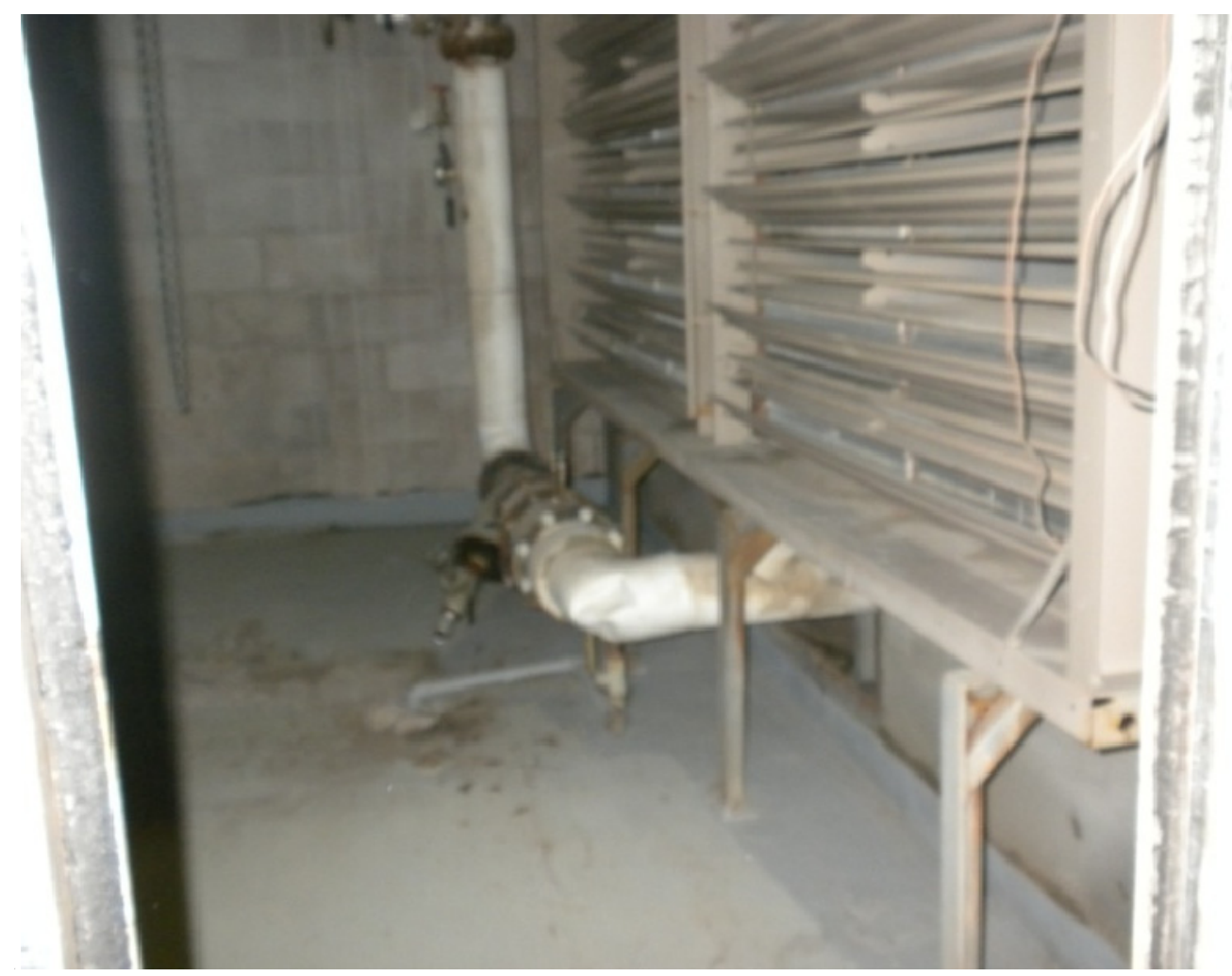

Figure 4. Old WHRS piping to the downstream side of the face and bypass damper in HVS-1. 


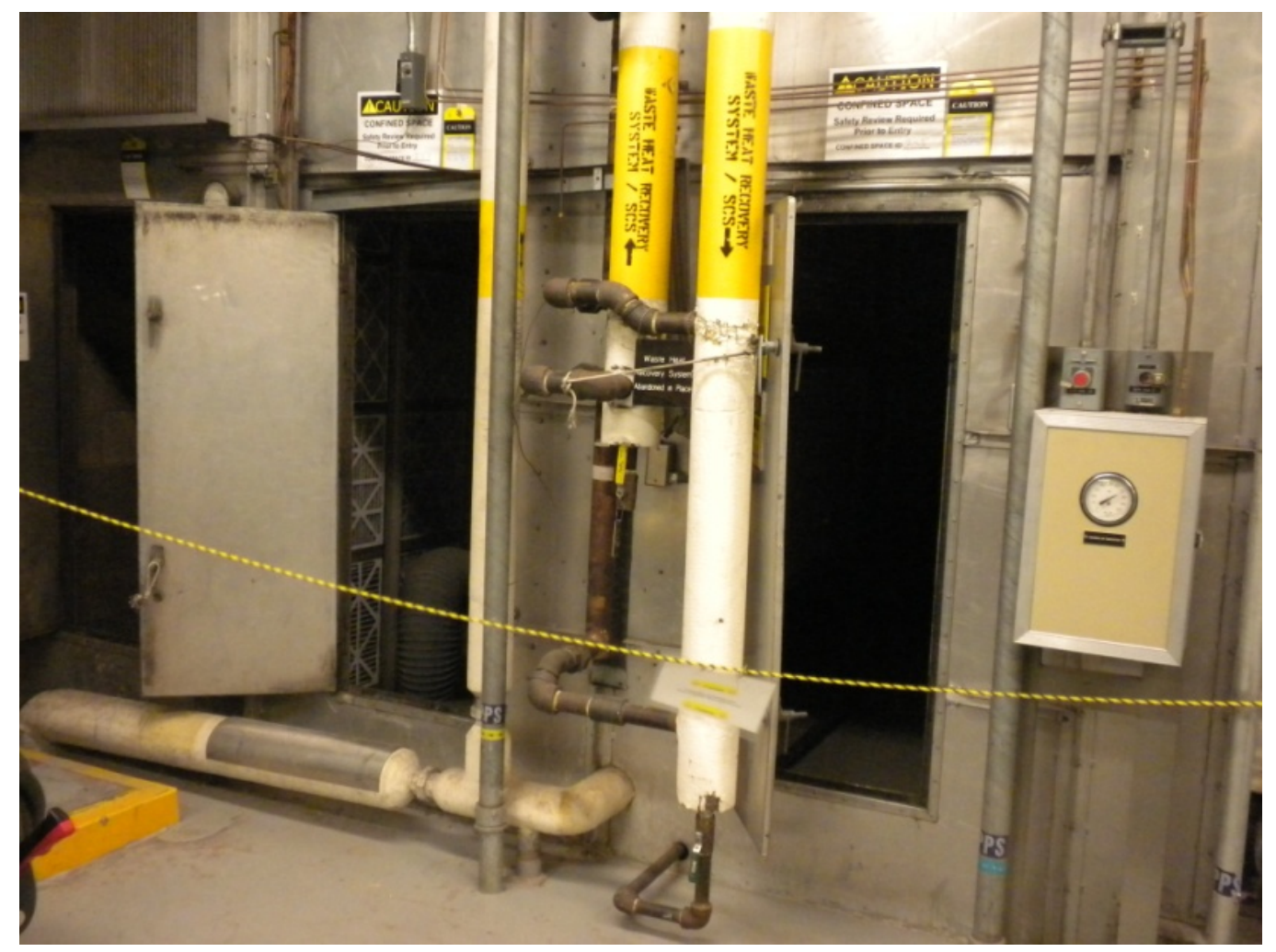

Figure 5. Old WHRS piping to HVS-5.

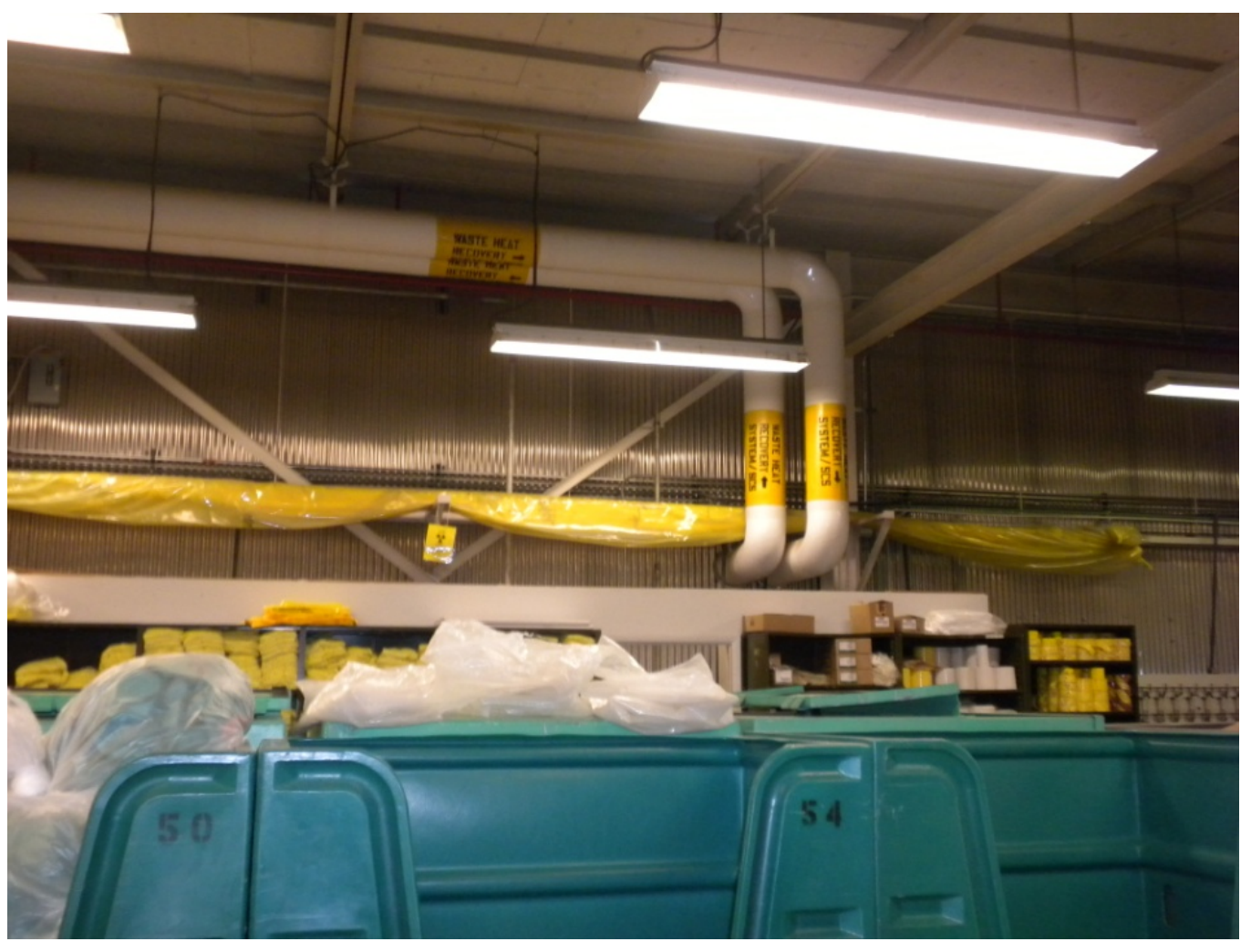

Figure 6. Old WHRS piping in TRA-670 layup area. 


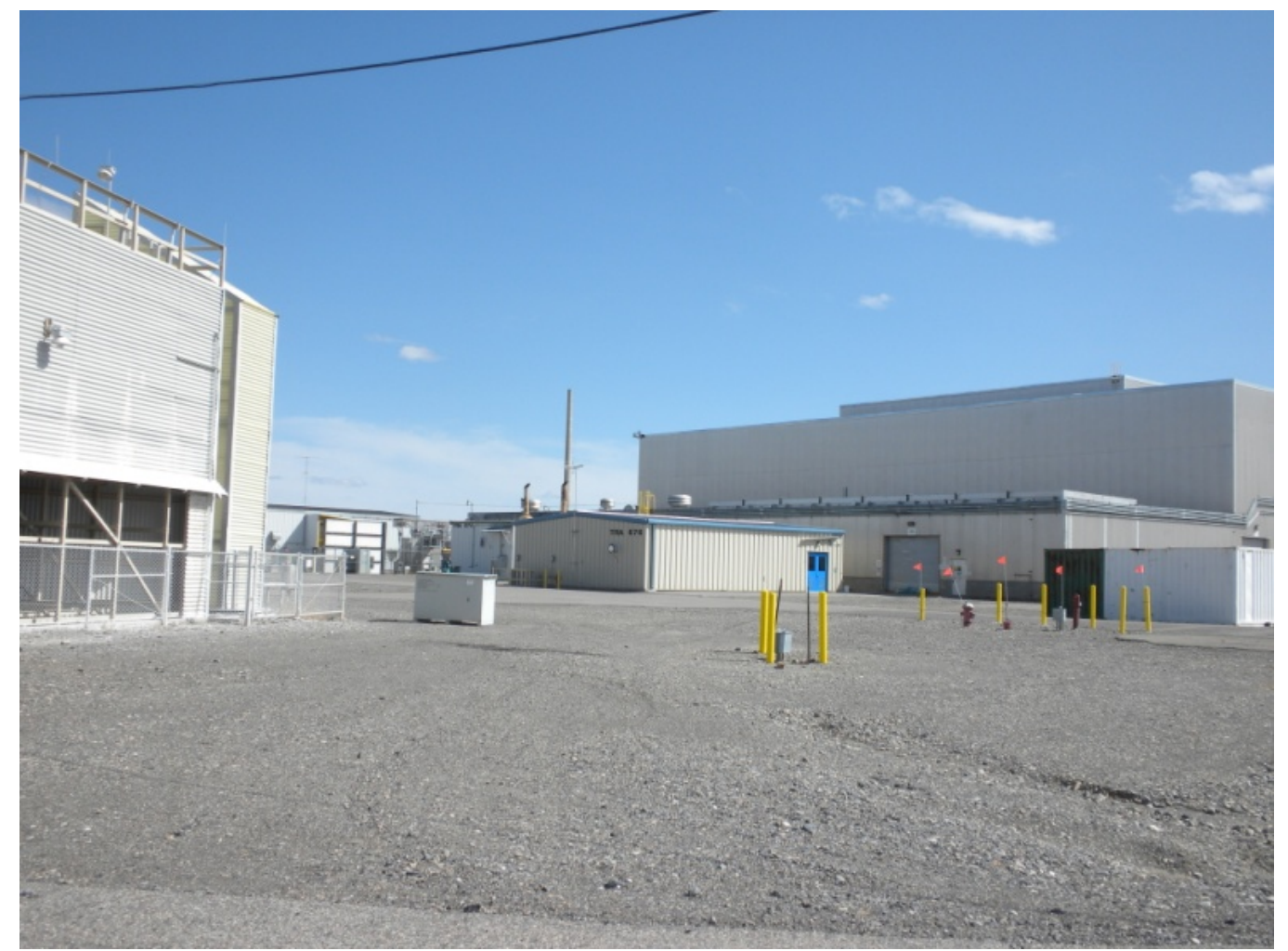

Figure 7. Old WHRS pump house (TRA-676, presently the fitness facility) on north side of TRA-670. 


\section{COMMENTS ON PROCESS FLOW DIAGRAM}

The Ameresco PFD, shown in Figure 1 above, indicates four HVS units, whereas there are actually five HVS units shown on Drawing 120281 for the ATR reactor building (TRA-670). Design air flows indicated on the drawing are compared with actual measured values in Table 1 . The air flow rates listed on the drawing are design values, whereas the actual values are much lower. Figure 8 shows the air intakes for HVS-1 (upper grill) and HVS-2 (lower grill). The intake grills for HVS-3, -4 and -5 units, shown in Figure 9, are located on the east side of TRA-670. Air intakes for HVS-3 and HVS-4, which are combined, are located near the top of the photo. HVS-5 is located at the right side of Figure 9.

Table 1. HVS air flow rates for TRA-670.

\begin{tabular}{|ccc|}
\hline \multirow{2}{*}{ HVS } & \multicolumn{2}{c|}{ cubic feet per minute (cfm) } \\
\cline { 2 - 3 } Unit & Design & Actual \\
\hline 1 & 64,800 & $55,000^{*}$ \\
2 & 12,500 & 10,000 \\
3 & 23,500 & 21,000 \\
4 & 13,500 & 62,000 \\
5 & 79,450 & 148,000 \\
\cline { 2 - 3 } Total & 193,750 \\
\hline \multicolumn{3}{|c}{} \\
\hline
\end{tabular}

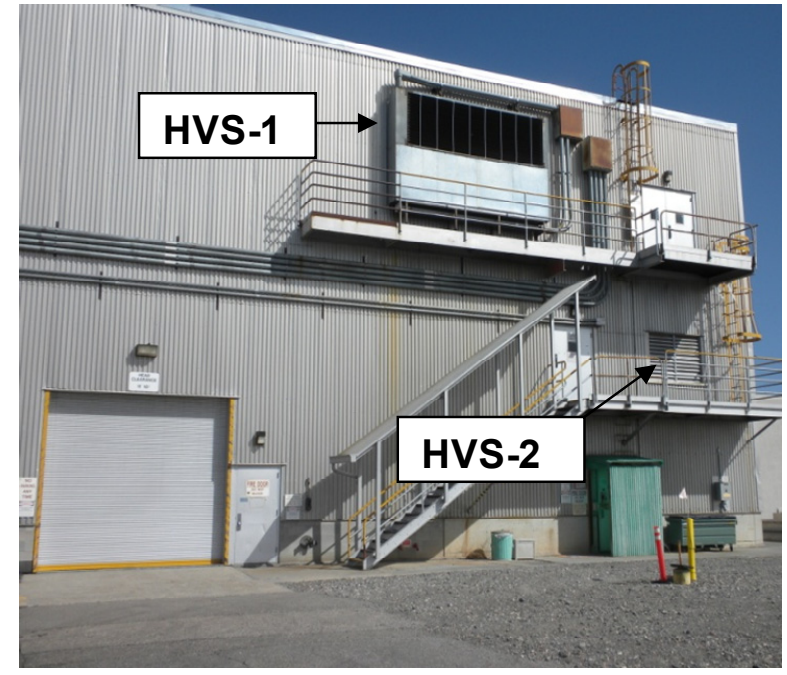

Figure 8. HVS-1 and -2 air intakes on the west side of TRA-670.

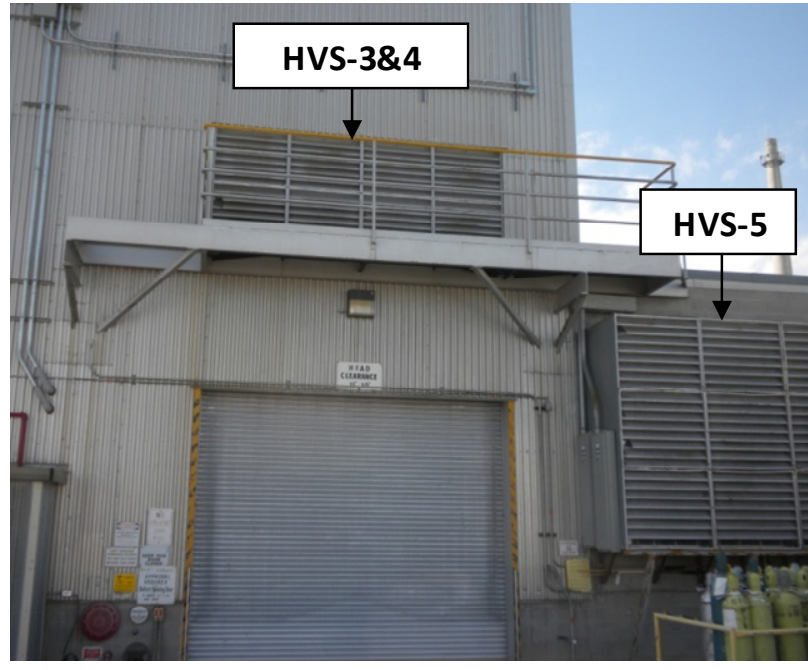

Figure 9. HVS-3, -4 , and -5 air intakes on the east side of TRA-670.

The drawings show that the heat exchanger coils from the old WHRS were abandoned in place and remain in the ducts of HVS-1, $-3,-4$, and -5 units. It is possible that flow blockage from the old coils is reducing the air flow. The actual flow rates should be measured to provide accurate data for the design of the g-WHRS and to ensure that industrial hygiene standards are being met. Regardless of whether the decision is made to proceed with the g-WHRS, removal of the old WHRS coils may save energy costs and make it easier to perform maintenance on the existing HVS units. If the g-WHRS coils are installed without removing the old coils, a larger fan capacity could be required because of the added pressure drop 
from the g-WHRS coils placed in the flow path. It is likely that the old coils are resulting in higher energy costs to operate the fans. Removal of the old WHRS piping associated with these coils that is abandoned in place is recommended before installing piping associated with a new system. Adding piping without removal of the old piping would increase the loading on the building structure, requiring a structural evaluation to be performed.

If new coils are installed, the heat exchange area should be sized such that the air flows to the building do not drop below industrial hygiene standards. If higher capacity fans are needed, the additional power costs relative to energy savings should be assessed, along with the ability of the existing transformers, etc., to handle the increase in power needed for the new higher capacity fans.

It is assumed that $100 \%$ outside air is supplied to HVS-1, $-2,-3,-4$, and -5 . If installed, the g-WHRS heating coils should be placed downstream of the existing electrical resistance preheating coil to prevent buildup of ice/hoarfrost on coils. The INL Site is notorious for hoarfrost and it has been known to build up on equipment exposed to the elements. The existing electrical preheaters would be used to preheat the air to $20^{\circ} \mathrm{F}$ before flowing past the g-WHRS coils. The g-WHRS would be used to heat the air from $20^{\circ} \mathrm{F}$ to $65^{\circ} \mathrm{F}$. Existing electrical duct burners would still be needed to supply heat during ATR outages.

\subsection{New g-WHRS System}

The proposed g-WHRS system consists of a new tertiary coolant loop that takes a side stream off of the main SCS loop. The SCS header pit, located on the north side of TRA-670, is shown in Figure 10. Heat from the SCS coolant water is transferred to a glycol loop by means of a plate and frame heat exchanger. The glycol loop would be used to preheat outside air in HVS units at TRA-670.

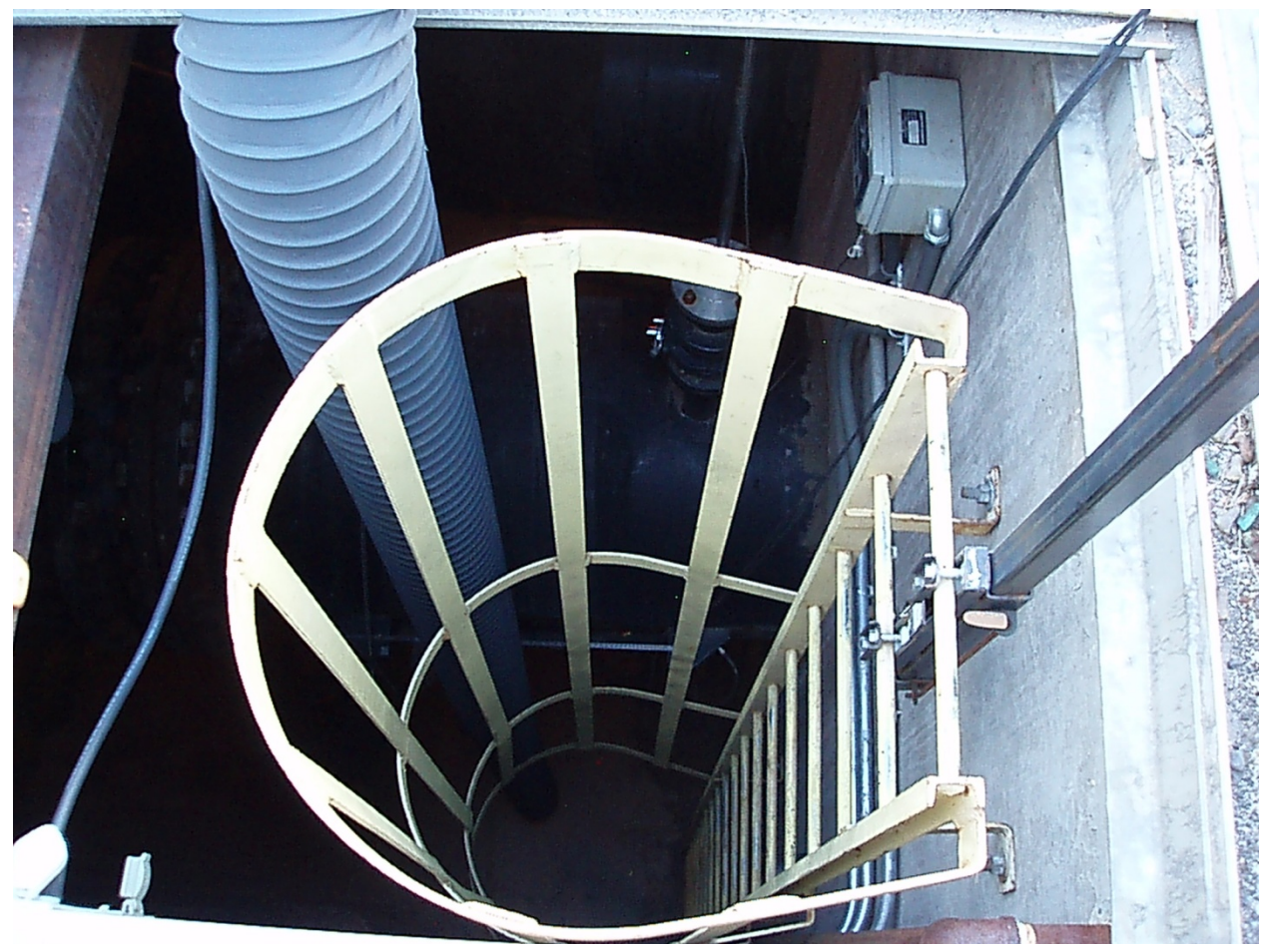

Figure 10. ATR SCS header pit located on north side of TRA-670. 


\subsection{Proposed Tertiary Coolant Loop}

Figure 11 shows the heat and coolant flows in the proposed g-WHRS. The proposed new plate and frame heat exchanger between the secondary coolant loop and the tertiary glycol loop must be located within an enclosure where it is protected from humidity, frost, snow, etc. Plate and frame heat exchangers typically have heat transfer coefficients 3 to 4 times that of shell and tube heat exchangers, but would require periodic maintenance to remove fouling and scale buildup on the heat exchanger surfaces, especially those in contact with the SCS water.

The proposed tertiary loop off of the main SCS loop requires freeze protection and heat trace lines to prevent freezing. Costs for this pumping power must be factored into the cost-benefit assessment. Because air ingress can cause corrosion in heat exchangers and piping systems, draining the tertiary flow system during outages is not recommended. A method of isolating the heat exchanger from the flow loop is necessary.

H\&V preheat system

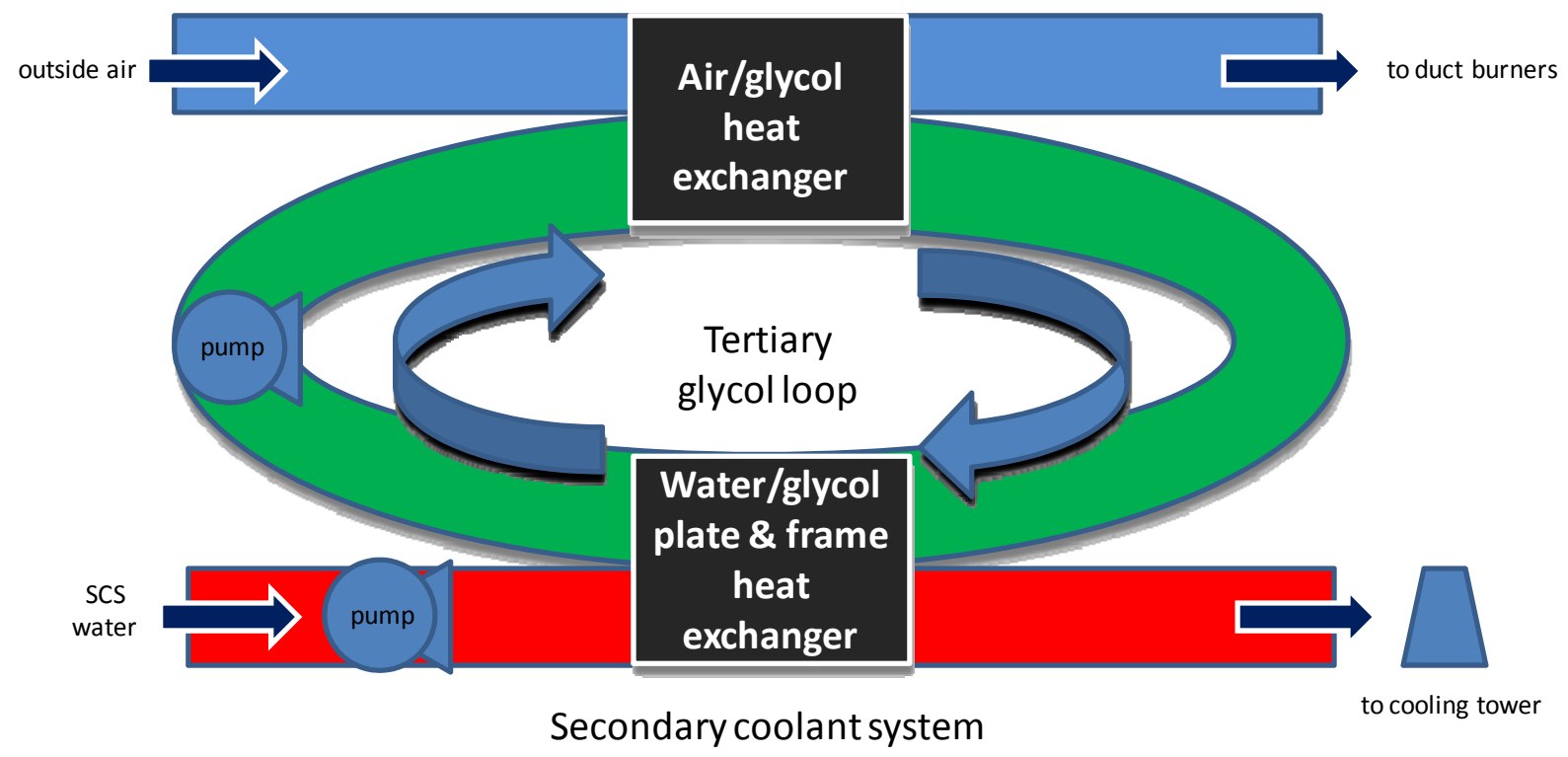

Figure 11. Heat and coolant flows in proposed g-WHRS.

The effects of the new system on cooling tower performance should be assessed. The four-cell, induced-draft cooling tower at ATR is shown in Figure 12. Changes to operating procedures, especially during the winter, may be required. Also, additional demands on personnel to operate and maintain this system must be considered and factored into the budget. 


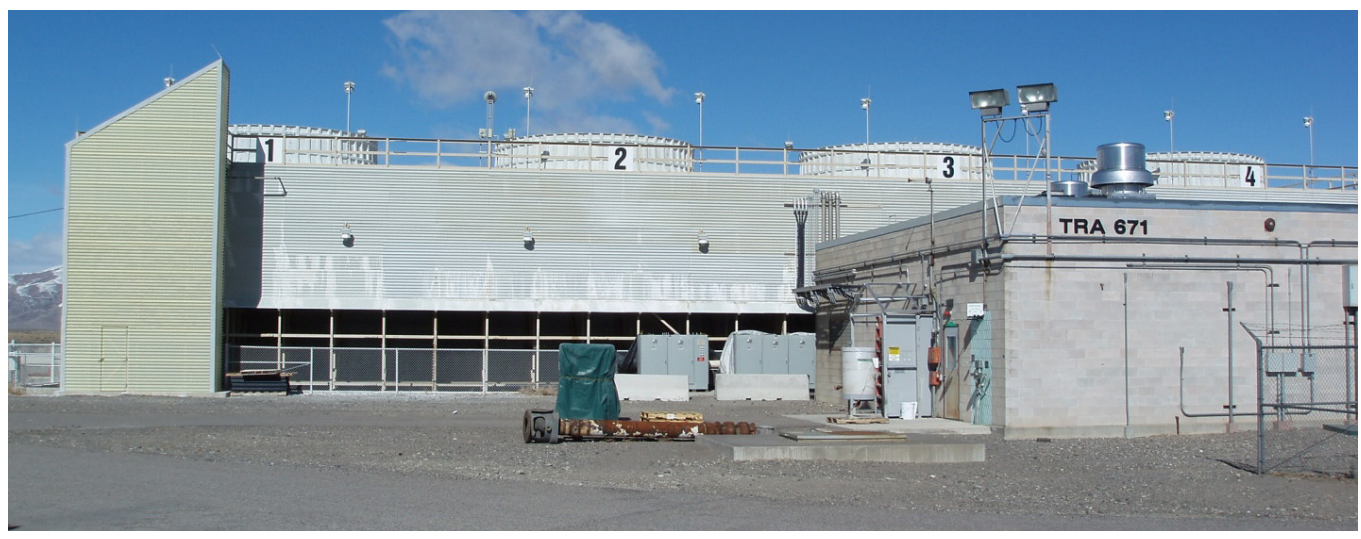

Figure 12. ATR four-cell cooling tower.

\subsection{Glycol Loop Operation and Logistics}

The type of glycol used would have to be propylene glycol $\left(\mathrm{C}_{3} \mathrm{H}_{8} \mathrm{O}_{2}\right)$, or possibly a renewable corn glycol. In any case, because of toxicity and environmental concerns, ethylene glycol (even though it exhibits better heat transfer performance) should not be used at INL. A $40 \%$ water- $60 \%$ propylene glycol solution would provide freeze protection to $-55^{\circ} \mathrm{F}$. There is no flash point specified for the glycol solution, so flammability is not a concern. High quality glycol should be procured, since the use of cheaper fluids may ultimately end up costing more because of quicker fluid degradation, fouling of pipes and heat exchanger surfaces, etc. Use of a corrosion inhibitor is necessary for the propylene glycol-water solution. The water used in the glycol systems must be of the highest possible quality (softened, demineralized, deionized), otherwise the hardness and corrosive salts in most domestic water supplies will deplete the inhibitor concentration in the glycol. The water-glycol solution quality should be checked at least annually.

Since propylene glycol increases major head loss in the heat recovery system, pumping power would be greater than that needed for a water system. The glycol loop must accommodate thermal expansion (pressure relief valves, surge tank, etc.) per piping code, and this equipment must be factored into the cost estimate. Water/glycol lines must be located a sufficient distance away from potable water lines. It must be verified that existing transformers could handle the additional pumping power needed for the glycol system.

An acceptable location for the g-WHRS equipment (pipes, pumps, etc.) would have to be found. There is potentially space available on the northwest side of the reactor building, but this may create difficulties for other ATR activities, such as primary heat exchanger replacement. Construction in certain areas (e.g., within $2 \mathrm{ft}$ of a pressurized line) can only take place during a reactor outage. Building penetrations that breach the confinement can only be performed during an outage.

During ATR outages, the glycol loop must be isolated so that it does not serve to cool the SCS. Consequences of periodically shutting down the glycol loop and having the fluid sit in it without flowing should be addressed. Frequent startup and shutdown tends to be hard on mechanical equipment, and this should be factored into maintenance requirements. An operator would be needed to shut down the glycol loop during reactor outages; otherwise, the glycol will serve to cool the secondary coolant. Continuing resources must be allocated to support this activity. 


\section{SAFETY CONSIDERATIONS}

The ATR is a Department of Energy (DOE)-owned Hazard Category 1 nuclear facility whose principal function is to provide a high neutron flux for irradiation testing of reactor fuels and other materials. Any modifications to the SCS must undergo review to ensure they do not present (a) an unreviewed safety question, (b) a threat to the health and safety of workers or the public, (c) adverse environmental effects, or (d) a hazard to the reactor facility or other equipment. Postulated accident scenarios must be formally analyzed using INL's formal procedures, and a determination made whether they are bounded by the events already addressed by the current safety analyses. The old WHRS was not an authorization safety basis system, nor was it mentioned in the ATR Upgraded Final Safety Analysis Report (UFSAR) [Grover and Brower 2003].

Accident events must be bounded by the sequences discussed in SAR-153 (2011), Chapter 15, Section 15.1.1. Applicable sections of the UFSAR include SAR-153, Chapter 15, Section 15.1, "Increase in Heat Removal by the Secondary (Cold Water Addition)" and Section 15.2, "Decrease in Heat Removal by the Secondary." A complete and abrupt loss of secondary coolant stemming from a piping failure upstream of the heat exchangers is the enveloping event for degradation of the heat sink. The supporting analyses for the complete loss of heat sink events are documented in EDF-5522 (Bayless and Polkinghorne 2005). Loss of SCS inventory because of a piping failure in the WHRS is enveloped by the primary coolant system loss of heat sink analysis. Reviewers must ensure that any modifications made to the SCS are bounded by the events addressed by the SAR. A determination would have to be made whether the existing analysis bounds events that could occur because of installation of the new system. Other sections of the UFSAR that may be affected by the g-WHRS include Chapter 9, Section 9.2, "Auxiliary Water Systems," and Section 9.4, "Heating and Ventilation." Examples of new events to consider are:

1. Water/glycol leak from/into SCS. What does it do to heat transfer characteristics, pumping power, and interaction with biofouling chemicals?

2. Flooding from a leak caused by a break in the tertiary glycol piping or heat exchange system. 


\section{USE OF WASTE HEAT FOR SPACE HEATING}

\subsection{Typical Energy Usage}

The most efficient use of waste heat is to use it directly as heat. This avoids efficiency losses that occur when heat is transformed to another kind of energy. Heat recovery is most effective when the heat source and heat sink are coincident, meaning they are physically close together and occur at the same time. The recovered heat can be used for space heating.

Commercial buildings and homes account for $40 \%$ of all energy use in the U.S. The U.S. is responsible for $20 \%$ of the world's carbon dioxide emissions, with energy usage by U.S. buildings responsible for $8 \%$. As shown in Figure 13, space heating accounts for approximately $13.7 \%$ of the energy usage in a typical commercial building (U.S. DOE, 2010). Climatology data for Idaho (U.S. DOC, 2011) indicates there were 6,867 degree days during 2010 (base temperature of $65^{\circ} \mathrm{F}$ ).

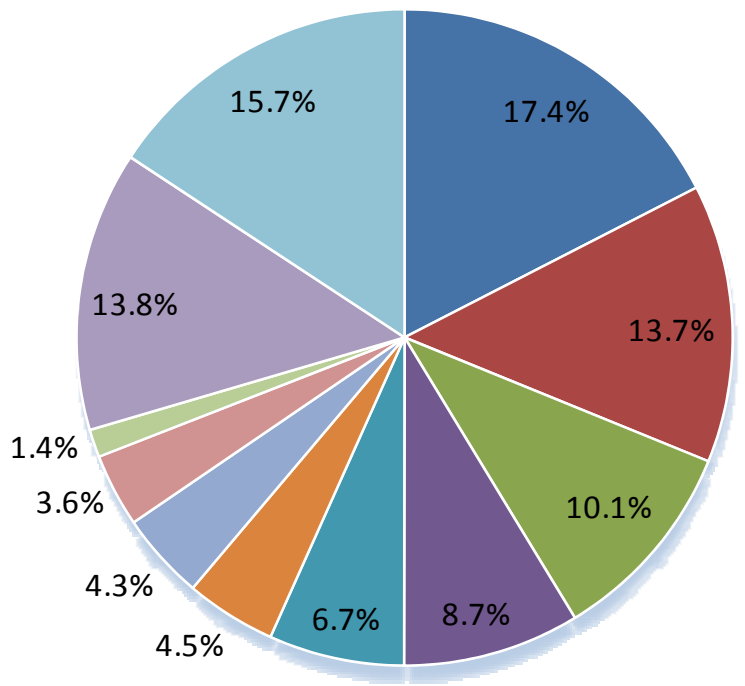

$$
\begin{aligned}
& \square \text { Lighting } \\
& \square \text { Space Heating } \\
& \square \text { Space Cooling } \\
& \square \text { Ventilation } \\
& \square \text { Refrigeration } \\
& \square \text { Electronics } \\
& \square \text { Water Heating } \\
& \square \text { Computers } \\
& \square \text { Cooking } \\
& \square \text { Other } \\
& \square \text { Energy Adjustment }
\end{aligned}
$$

Figure 13. Commercial building energy usage splits (U.S. DOE, 2010).

\subsection{Availability of SCS Waste Heat}

Since the ATR is a materials and test reactor rather than a commercial power reactor, it is frequently shut down for outages. A hybrid system wherein the electrical heating takes over when the reactor is shut down would therefore be required to preclude freezing. The existing electrical preheaters and duct burners in the HVS units would still be required. Energy savings from using SCS waste heat would only be realized during reactor operation in nonsummer months. Outages lasting approximately 2 weeks occur every 6 weeks, with a longer 6-week outage taking place every 18 months. A 6-month shut down for core internals change-out is scheduled once every 10 years. The historical data of actual (rather than planned) ATR operating cycles over the past 10 years is shown in Table 2. The ATR has been operating 55.7\% of the year on average, and only $43.5 \%$ of the year when heating is needed (October through June). 
Table 2. ATR full-power hours over the past 10 years (source: Marjorie Owens, ATR).

\begin{tabular}{lrrrrrrrrrr}
\hline & \multicolumn{10}{c}{ ATR Full-Power Hours } \\
\cline { 2 - 11 } & $\mathbf{2 0 0 2}$ & $\mathbf{2 0 0 3}$ & $\mathbf{2 0 0 4}$ & $\mathbf{2 0 0 5}$ & $\mathbf{2 0 0 6}$ & $\mathbf{2 0 0 7}$ & $\mathbf{2 0 0 8}$ & $\mathbf{2 0 0 9}$ & $\mathbf{2 0 1 0}$ & $\mathbf{2 0 1 1}$ \\
\hline Jan & 583.6 & 514.9 & 485.3 & 0.3 & 546.3 & 744.0 & 474.2 & 700.0 & 531.3 & 178.0 \\
Feb & 668.0 & 564.4 & 94.8 & 55.7 & 511.9 & 294.1 & 593.8 & 466.0 & 399.3 & 0.0 \\
Mar & 443.2 & 349.0 & 744.0 & 0.0 & 197.1 & 673.3 & 178.0 & 456.5 & 743.0 & 0.0 \\
Apr & 719.0 & 710.3 & 411.0 & 387.6 & 324.0 & 489.0 & 0.0 & 585.9 & 289.9 & 389.7 \\
May & 264.0 & 346.4 & 403.4 & 617.6 & 739.5 & 0.0 & 709.7 & 498.5 & 704.6 & 490.0 \\
Jun & 429.9 & 542.3 & 720.0 & 198.7 & 295.7 & 137.0 & 436.2 & 653.4 & 188.8 & 573.3 \\
July & 742.1 & 611.5 & 494.9 & 369.8 & 215.0 & 609.0 & 556.5 & 82.0 & 687.2 & 706.0 \\
Aug & 408.7 & 261.9 & 155.0 & 588.9 & 0.0 & 0.0 & 666.2 & 0.0 & 322.0 & 286.1 \\
Sept & 519.1 & 0.0 & 0.0 & 504.8 & 0.0 & 470.4 & 157.0 & 571.1 & 716.9 & 3.7 \\
Oct & 745.0 & 0.0 & 0.0 & 745.0 & 464.1 & 381.5 & 372.1 & 600.0 & 416.6 & 390.2 \\
Nov & 246.6 & 60.1 & 0.0 & 261.5 & 720.0 & 721.0 & 494.0 & 292.1 & 301.3 & 611.0 \\
Dec & 744.0 & 557.9 & 0.0 & 744.0 & 345.0 & 379.8 & 329.7 & 669.7 & 744.0 & 342.9 \\
Total Hours & 6513.2 & 4518.5 & 3508.4 & 4473.8 & 4358.6 & 4899.1 & 4967.3 & 5575.2 & 6044.9 & 3970.8 \\
Total Days & 271.4 & 188.3 & 146.2 & 186.4 & 181.6 & 204.1 & 207.0 & 232.3 & 251.9 & 165.5 \\
\% of Year & 74.4 & 51.6 & 40.1 & 51.1 & 49.8 & 55.9 & 56.7 & 63.6 & 69.0 & 45.3 \\
\% Yr Htg & 55.3 & 41.6 & 32.6 & 34.4 & 47.3 & 43.6 & 41.0 & 56.2 & 49.3 & 34.0 \\
\hline
\end{tabular}




\section{ENERGY AND COST/BENEFIT ANALYSIS}

The g-WHRS could recover some heat energy from the ATR SCS that would otherwise be lost as waste heat discharged at the cooling tower. The motivation for this study is to reduce energy consumption, carbon footprint, and costs. This section includes the possible yearly energy savings from installing a g-WHRS and estimated costs to install the system.

\subsection{Energy Savings}

The following calculation indicates that the proposed g-WHRS could save approximately $\$ 285 \mathrm{~K}$ per year in electricity costs. This calculation assumes a flat rate electricity price of $\$ 0.035 / \mathrm{kWh}$.

Properties of air

$$
\begin{aligned}
& \rho_{\text {air }}:=0.076 \frac{\mathrm{lb}}{\mathrm{ft}^{3}} \quad \Delta \mathrm{T} \text { air }:=45^{\circ} \mathrm{R} \quad \begin{array}{l}
\text { Preheat air by } 45 \mathrm{~F} \text {. These param } \\
\text { are based upon original WHRS }
\end{array} \\
& c \mathrm{p}_{\text {air }}:=0.24 \cdot \frac{\mathrm{BTU}}{1 \mathrm{bm} \cdot{ }^{\circ} \mathrm{R}} \quad \begin{array}{l}
\text { reasonable size H\&V coils. If } \\
\text { preheating was increased, so would }
\end{array} \\
& \text { heat exchange area (i.e., size) of coils. } \\
& \text { mdot }_{\text {air }}:=\rho_{\text {air }} \cdot 148000 \cdot \mathrm{cfm}
\end{aligned}
$$

Heat supplied by preheat system $43.5 \%$ of year

$$
Q_{\text {preheat }}:=0.435 \text { mdot }_{\text {air }} \cdot \mathrm{cp}_{\text {air }} \cdot \Delta \mathrm{T} \text { air }
$$$$
Q_{\text {preheat }}=2.779 \times 10^{10} \cdot \frac{\mathrm{BTU}}{\mathrm{y}^{\mathrm{r}}}
$$

Apply blended rate for INL electricity costs

$$
\begin{aligned}
& \text { Energycost }:=0.035 \cdot \mathrm{KwV}_{\overline{\mathrm{N}} \cdot \mathrm{hr}}^{\mathrm{x}} \\
& \text { Savings } \text { preheat }:=Q_{\text {preheat }} \cdot \text { Energycost } \quad \text { Savings }_{\text {preheat }}=285084 \cdot \underset{y^{1}}{\$}
\end{aligned}
$$

The proposed WHRS could possibly qualify for energy conservation credits or incentives that would offset capital or operating and maintenance costs. The use of a renewable propylene glycol (such as corn glycol) could potentially qualify for renewable energy credits. Electricity for the INL Site is derived from a mix of hydropower and fossil energy, which varies, depending upon the availability of hydropower in any given year. 


\subsection{Installation, Operating, and Maintenance Costs}

A Level 5 cost estimate was prepared for the proposed g-WHRS. The complete cost estimate with details is provided in Appendix A. Total project costs are estimated at $\$ 9.68 \mathrm{M}$. A management reserve of $35 \%$ has been applied. It is assumed that the funding would be supplied as indirect funding. The cost estimate includes the capital costs for equipment, installation, construction, demolition, and removal of abandoned lines that are in the way, as well as operating and maintenance costs for the first 5 years of operation. Table 3 outlines the total project costs. The execution phase has the highest costs, which are further delineated in Table 4. Figure 14 shows the relative percentage of the various costs. The most costly items are:

- Installation of the new piping, pumps, heat exchangers and ancillary equipment

- Construction of the new pump and heat exchanger building

- Pumps, heat exchangers and associated equipment (including spares) for the water and glycol loops

- Operations, including development of procedures and plans.

Table 3. Overall Level 5 estimated project costs.

\begin{tabular}{|c|c|}
\hline Item & Cost \\
\hline Project Management & $\$ 584,488$ \\
\hline Construction Management & $\$ 462,971$ \\
\hline Engineering Design & $\$ 705,514$ \\
\hline Design Plans, Reports \& Documents & $\$ 281,733$ \\
\hline Execution & $\$ 5,314,843$ \\
\hline Environmental Assessment & $\$ 171,007$ \\
\hline Operations & $\$ 968,018$ \\
\hline SAR Update & $\$ 676,639$ \\
\hline Readiness & $\$ 163,116$ \\
\hline New System SO Testing & $\$ 52,580$ \\
\hline \multirow[t]{2}{*}{ Project Closeout } & $\$ 299,638$ \\
\hline & $\$ 9,680,547$ \\
\hline
\end{tabular}

Table 4. Cost details for execution phase.

\begin{tabular}{|c|c|}
\hline Item & Cost \\
\hline Removal of Abandoned Equipment & $\$ 373,021$ \\
\hline Heat Exchangers, Pumps, Piping, Electrical \& Controls & $\$ 994,798$ \\
\hline Pump \& Heat Exchanger Building & $\$ 975,086$ \\
\hline Construction Support \& Oversight & $\$ 790,989$ \\
\hline General, Piping \& Electrical Contractor & $\$ 470,608$ \\
\hline Installation of Piping \& Equipment & $\$ 1,490,501$ \\
\hline Tie-in to SCS & $\$ 219,840$ \\
\hline Total for Execution Phase & $\$ 5,314,843$ \\
\hline
\end{tabular}


Overall Project costs

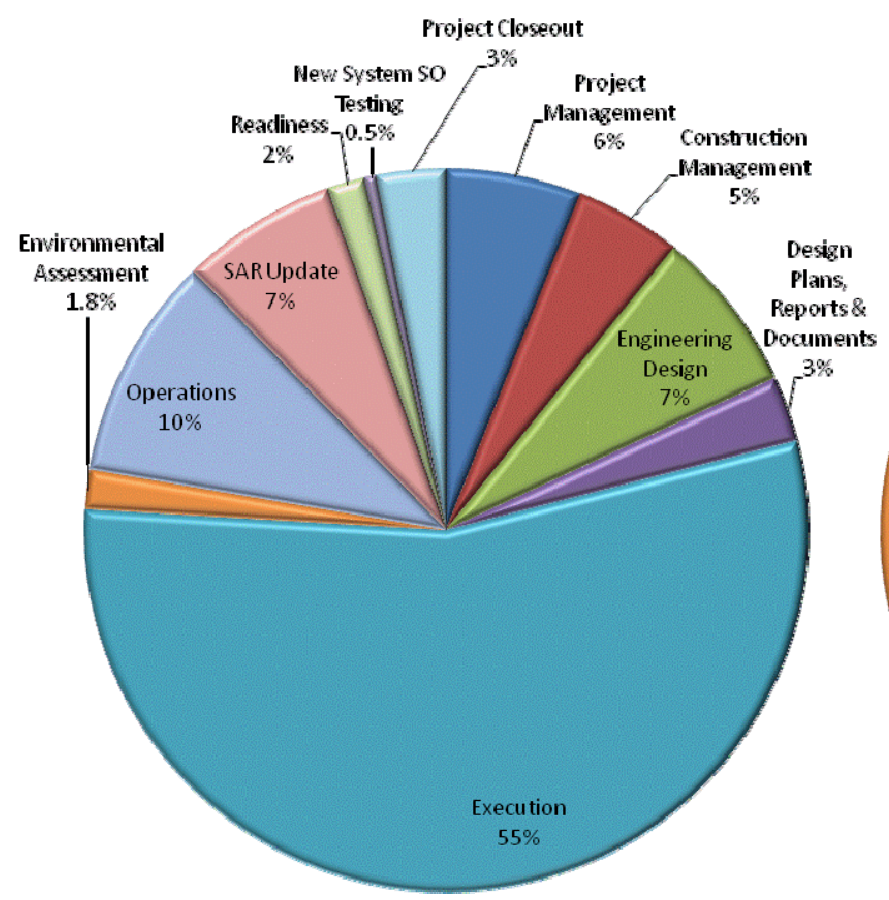

Execution Phase Costs

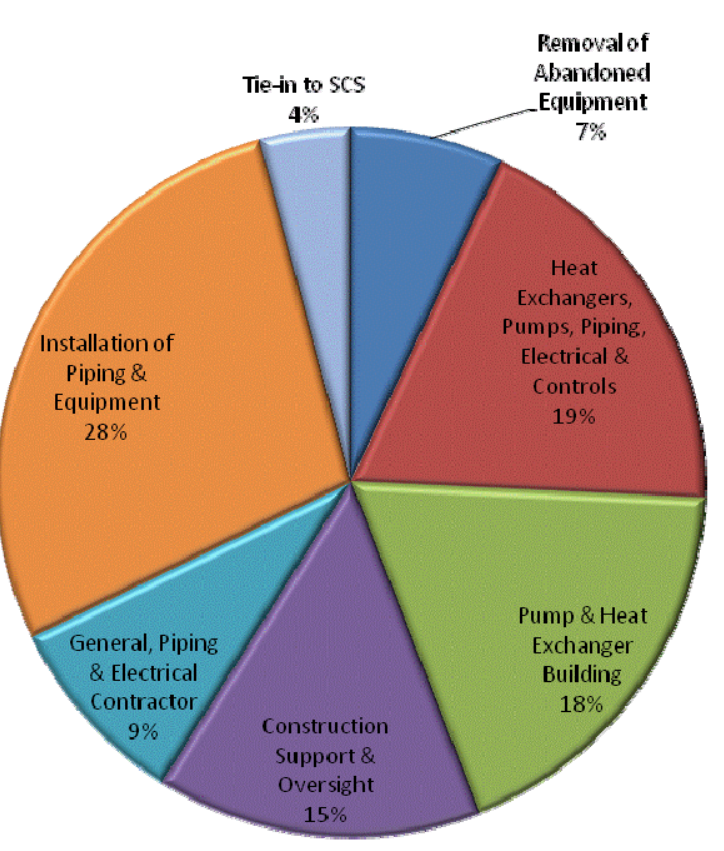

Figure 14. Proposed g-WHRS cost breakdown.

The cost estimate was based upon an ideal schedule for a consecutive 9-month duration for demolition, construction, and installation activities at the reactor building. Unforeseen delays would increase costs. Vacuum excavation is necessary to minimize the risk of damage to underground utilities. Demolition of the old WHRS entails removal of the old piping, insulation, coils, and associated equipment, with the exception of the existing pipe supports that would be reused. Existing penetrations would be used for the new g-WHRS piping. As can be seen in the photos of the canal area, the pipe supports are approximately $40 \mathrm{ft}$ above ground level, which requires the use of a snorkel lift for removal of the old piping and installation of the new piping. No construction debris will be allowed to fall into the canal, so appropriate measures must be put in place.

\subsection{Technical, Safety, and Logistics Considerations}

The following concerns must be addressed before proceeding with the g-WHRS concept. Issues in three areas have been identified: g-WHRS operational concerns, installation issues, and safety reviews and operating procedures.

\subsubsection{G-WHRS Operational Concerns}

Operation of the proposed g-WHRS imposes additional burdens on ATR Operations personnel, which will not improve the safety of the ATR but will increase ATR operating risks. The risks may outweigh any potential cost or energy-savings benefits. The following issues related to the operation and maintenance of the g-WHRS must be addressed:

1. Startup/shutdown of the proposed g-WHRS will coincide with the reactor startup/shutdown. The ATR's most significant event of 2011 occurred during preparation for reactor startup. How will operation of the g-WHRS impact ATR Operations? How much of the operators' time and attention will be required? 
2. Shutdown of the proposed g-WHRS will occur during reactor shutdown when ATR Operations personnel may be busier than during reactor startup. During planned shutdowns, most activities are planned and scheduled based upon the available personnel, resources, and time. During an unplanned reactor outage (a reactor scram due to events beyond the control of ATR Operation personnel, such as during a loss of commercial power), there is often no advance warning. The reactor immediately shuts down and the heat generation provided to the SCS drops to zero in seconds. During this time, personnel are extremely busy working to keep the reactor safe. Adding additional burdens to take actions to shutdown the g-WHRS would be an extremely inappropriate use of available personnel. Following a reactor scram, forcing ATR Operations personnel to make a decision between keeping the ATR reactor core safe or shutting down the g-WHRS is not a wise choice. If the operators do not take action to ensure the g-WHRS is shutdown, then it will affect SCS, primary coolant system (PCS), and core temperatures. Operator action to shutdown the g-WHRS would be critical during cold weather, when the g-WHRS is in service.

3. Does the g-WHRS need to be placed into a "wet lay-up" condition for those months when the system is not used? If so, what will be required to transition from wet lay-up to operating status? Will debris settle in the system?

4. What equipment or personnel actions are required to control ATR building ventilation temperatures when starting up or shutting down the g-WHRS? One of the complaints from the previous WHRS installed at the ATR Complex was that when the WHRS was operating, building temperatures were too hot. When the WHRS shut down, the temperatures were too cold. How much operator effort will be required to monitor building temperatures during startup and shutdown of the g-WHRS?

5. How much operator attention will be required to monitor the g-WHRS during steady state operations? Will operator rounds be required to check building temperatures and the g-WHRS equipment? Will operators need to take logs on the g-WHRS equipment?

6. How will the operators know if conditions are correct to startup the g-WHRS? Are there temperature limits for when the system should or should not be operated? What if the nighttime low temperature is $40^{\circ} \mathrm{F}$ and the daytime high temperature is $80^{\circ} \mathrm{F}$ ? Does the g-WHRS need to be shutdown every morning and restarted every evening? This could be a significant burden on ATR Operations personnel.

7. Does the glycol chemistry remain the same after periods of inactivity? Who will be responsible for sampling the glycol coolant? Who will perform the analysis of the glycol coolant? Who will evaluate the results of the analysis? Who will be responsible for taking action to make the necessary corrective actions to maintain the glycol coolant?

8. Who will be responsible for establishing the preventative maintenance procedures for the g-WHRS? Who will responsible for performing the maintenance? Who will pay for the replacement parts and materials?

9. Used glycol cannot be discharged to the evaporation pond. What is the proposed disposition pathway for low-level radioactively contaminated glycol?

\subsubsection{Installation Considerations}

Retrofitting an existing coolant loop is much more difficult than designing a WHRS that is an integral part of a new system. Installation of the proposed g-WHRS represents a significant investment in a new structure and equipment; it includes:

1. Providing necessary instrumentation and controls for the hybrid electrical/g-WHRS. Upgrades to other equipment (electrical, instrumentation, control systems, etc.) may be required to bring these 
systems into compliance with current codes when installing the new system. This decision is made by the authority having jurisdiction.

2. Routing piping for the proposed g-WHRS in the same locations as the old WHRS piping, which has been abandoned in place and its condition unknown. It is recommended that the piping from the previous WHRS that was abandoned in place be removed before installing the new piping associated with the g-WHRS. If additional new piping is routed without removing the previous WHRS piping, the loading affect on the ATR building structure will need to be evaluated. Removal of old steam and condensate lines may be complicated by the presence of asbestos insulation.

3. Maintaining the drawings of the g-WHRS system and keeping them current.

4. Performing the installation of the new coils when space heating is not required, so as not to adversely impact building occupants and operations.

\subsubsection{Safety Reviews and Operating Procedures}

Existing safety documentation must be reviewed and any unreviewed safety questions (USQs) addressed. Procedures must be established for normal and off-normal operations as follows:

1. The proposed glycol coolant creates a new flooding event that is different from any previously evaluated flooding event for ATR. Although the g-WHRS would not be installed within the confinement, there is the potential for flooding to impact vital systems, such as electrical equipment switchgear. This may have an effect on the ATR SAR-153, TSR-186, and Probabilistic Risk Assessment (PRA), which will need to be evaluated.

2. Operating documents will need to be created, maintained, and updated for startup, shutdown, monitoring, logkeeping, and maintenance of the g-WHRS. A procedure will need to be created to address personnel actions and cleanup requirements in the event of a glycol spill. 


\section{OTHER ENERGY AND WATER EFFICIENCY OPPORTUNITIES AT ATR}

It is paramount that any energy conservation measures (ECMs) considered for implementation must not adversely impact the mission of the ATR. The objectives of the ECMs should be to reduce energy or water waste, not use.

\subsection{Alternate Waste Heat Recovery Concepts}

Some alternatives to the g-WHRS for waste heat recovery at ATR are to recover heat from:

- The ATR diesel engine generators (DG). One of the two Enterprise DSQ-38 DGs operates continuously during reactor operation and for a minimum of 30 minutes after reactor shutdown to provide electric power for mission-critical vital loads. Each engine is an in-line, eight-cylinder, fourcycle diesel engine with a displacement of $32,170 \mathrm{in}^{3}$, rated for 2,170 horsepower at 360 revolutions per minute, and drives a $4,160 \mathrm{~V}_{\mathrm{ac}}, 1,500 \mathrm{~kW}$ generator (Ceci 2008).

- Heat generated during DG operations could be used for hot water needs or for space heating; freeze protection of water pipes would be required.

- Or, if the loads were instead configured to operate on commercial power, rather than diesel, there could be a cost-savings from reduced operating and maintenance costs and a reduction in greenhouse gas (GHG) emissions. The DGs would be kept as a backup and operated in the event of a loss of commercial power.

- Exhaust air flows using heat pipe heat exchangers, heat recovery ventilators, or energy recovery ventilators.

- Air conditioning system to provide preheated water to the water heater.

- Water-cooled air compressors for hot water preheating.

\subsection{INL Water Usage}

An assessment of water usage at INL (Cabe et al. 2012) provided four recommendations to improve water efficiency at the ATR:

1. Incorporate a cooling tower blowdown controller for total dissolved solids and $\mathrm{pH}$ control.

2. In conjunction with Recommendation 1, replace the current scale and corrosion control chemicals with materials that are less likely to precipitate as scale-forming constituents in the recirculating cooling tower water.

3. Eliminate once-through cooling water for the air-compressor heat exchangers and replace with aircooled heat exchangers. Drawbacks to this proposed concept are reduced reliability during hot weather operations and the need for water to be supplied to the ATR sewage lagoon to keep the bentonite liner wet.

4. Eliminate once-through cooling water for ATR heating, ventilation, and air conditioning (HVAC) equipment when the reactor is off-line.

For additional details, see Cabe et al.'s 2012 report. If all of the above recommendations were implemented, the estimated yearly savings would be $145.6 \mathrm{M}$ gal for water and $\$ 17.7 \mathrm{~K}$ for energy. These recommendations should be evaluated by ATR Operations personnel to determine impacts to the reactor and ancillary systems. 


\section{RECOMMENDATIONS}

The simple payback for the proposed g-WHRS is 35 years. This does not include operating and maintenance costs beyond the first 5 years of operations. Industry typically does not consider WHRSs as a capital improvement project, with a target payback within 3 years; rather, industry considers a WHR project with a payback period within 6 years to be acceptable (Tillman, 2012). The long payback period for the proposed g-WHRS is due to a combination of factors that tend to reduce energy savings and increase costs. These factors include the low cost of electricity, partial year operation and high cost of performing work in a Hazard Category 1 nuclear facility. The low temperature of the SCS makes it extremely unattractive for other waste heat applications, such as the generation of electricity via the Organic Rankine Cycle. The poor economics are compounded by operational concerns and previous bad experience with the old WHRS. Based upon these factors, the g-WHRS is not an attractive option for WHR at the ATR. 


\section{REFERENCES}

Bayless, P. D., and S. T. Polkinghorne, 2005, "Updated Complete Loss of Flow and Complete Loss of Heat Sink Analyses for the Advanced Test Reactor," Idaho National Laboratory Engineering Design File, EDF-5522, January 18, 2005.

Brown, R. D. and Donovan, L. E., 1979, "Conceptual Design Report for TRA Heating System Upgrade with Waste Heat Recovery,” EG\&G Idaho, Inc., Internal Technical Report No. RE-D-77-212, Rev. 2, March 5, 1979.

Cabe, J. E., De La Rosa, M. I., Boyd, B. K., Russell, B. W., McMordie Stoughton, K. L., 2012, "Idaho National Laboratory Water Assessment," Pacific Northwest National Laboratory Report PNNLXXXXX, February 2012.

Ceci, D. M., 2008, "Project Management and Technical Support Service for the ATR Enterprise Diesel Engine Overhauls," SOW-6743, September 25, 2008.

Grover, G., and J. Brower, 2003, "USQ Screen for Modifications to Nuclear Reactor Facilities," SES2002-457, Modification/FCF Number 8.1.2-6/6795 Rev. 1, March 14, 2003.

Kinnaman, T. L., 1993, “ATR Waste Heat Recovery System Energy Savings Validation Study,” EG\&G Idaho, Inc., Internal Technical Report No. 2610-93-077, November 1993.

SAR-153, 2011, "Upgraded Final Safety Analysis Report for the Advanced Test Reactor," Idaho National Laboratory Safety Analysis Report SAR-153, Chapter 5 - Primary Coolant System, Rev. 14, February 15, 2011.

Tillman, T., TAS Energy presentation to the Technology Forum on Low-Temperature Waste Energy Recovery in Chemical Plants and Refineries, Houston, Texas, May 16, 2012.

U.S. Department of Energy, Office of Energy Efficiency and Renewable Energy, Buildings Energy Data Book: 3.1 Commercial Sector Energy Consumption, March 2011.

U.S. Department of Energy, Office of Energy Efficiency and Renewable Energy, 2010, "Energy Efficiency Trends in Residential and Commercial Buildings," August 2010.

U.S. Department of Commerce National Oceanic and Atmospheric Administration National Environmental Satellite, Data, and Information Service, 2011, Historical Climatology Series 5-1, July 2009 through Dec 2010, Published March 2011. 
Appendix A

g-WHRS Cost Estimate 


\section{Appendix A}

\section{g-WHRS Cost Estimate}

\section{INTEROFFICE MEMORANDUM}

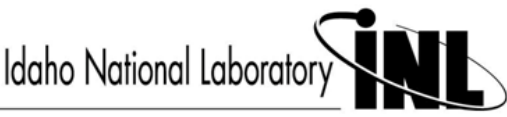

Date: $\quad$ May 9,2012

To:

C. P. Ischay, Engineer

From: $\quad$ A. W. Miller, S. N. Wasley Cost Estimators

Subject: RTC Waste Heat Recovery

Per your request, Cost Estimating prepared a cost estimate (Class 5) for the above-mentioned subject. The estimated cost, including escalation and management reserve, is $\$ 9,600,000$.

Please note the following:

A. Per the requester, this work will be indirect funded. General and Administrative (G\&A) costs are not included in this estimate.

B. This cost estimate has been evaluated in the Association for the Advancement of Cost Engineering International (AACEI) classification matrix as a Class 5 estimate (ref. Department of Energy (DOE) G. 430.1-1X, Appendix J). The primary characteristic used in this guideline to define the classification category is the degree of project definition at this time. The intent of this classification is to assist in the interpretation of the quality and value of the information available to prepare this cost estimate and the expected accuracy levels that can be produced. Per AACEI, a Class 5 indicates the lowest amount of project information quality and value with a graded approach to a Class 1 , which indicates the highest amount of project information quality and value.

C. A review of this cost estimate was held on May 8,2012, with you, the project team, and the cost estimators. This review allowed the estimators to discuss, in detail, the perceived scope, basis of estimates, assumptions, project risks, and the resources that make up this cost estimate. Comments from this review have been incorporated into this estimate to reflect a project team consensus of this document.

D. This project has been identified by Idaho National Laboratory (INL) Construction Management, R. E. Strong, as a "Block 2" in the INL Construction Commercial Practices Evaluation for the purpose of construction scope, management, and field oversight.

Refer to the cost estimating summary, detail, markup, and labor sheets with the cost breakdowns. Also included for your use are the cost estimate recapitulation sheets describing the basis and assumptions used in development of this estimate.

This estimate, the work, and the work breakdown structure are based on the information perceived by this estimator as to the scope of work to be completed. Any changes to the methodology used to prepare this estimate could have a significant effect on the cost estimate and/or schedule and should be reviewed by me. If you have any questions or comments, do not hesitate to contact me at 526-1827 or e-mail Andrew.Miller@inl.gov or Scott Wasley at 526-6835 or Scott.Wasley@inl.gov. 
C. P. Ischay

May 9, 2012

Page 2

\section{Attachments}

\section{cc: Estimate File 7B50}

Uniform File Code: $\underline{8309}$

Disposition Authority: A16-1.5-b

Retention Schedule: Cut off at the end of each fiscal year. Destroy 10 years after cutoff.

NOTE: Original disposition authority, retention schedule, and Uniform Filing Code applied by the sender may not be appropriate for all recipients. Make adjustments as needed.

Final Letter Template 32212 
Project Name: RTC Waste Heat Recovery

Project Location: $\boldsymbol{A I R}$

Project Number: 7850

ESTIMATE ELEMENT

Total Estim ated Cost (TEC)

Other Project Cost (OPC)

Total Cost

Rounded Total Cost (Rounded to the nearest $\$ 100000$ )
Summ ary Report

\begin{tabular}{|c|c|c|c|}
\hline \multirow[b]{2}{*}{$\frac{\text { Estlmate Subtotal }}{\$ 3,823,811}$} & \multicolumn{2}{|c|}{ Management } & \\
\hline & $\frac{\text { Escalation }}{\mathbf{8} 82 \%}$ & $\begin{array}{l}\text { Reserve } \\
3500 \% \\
\$ 1,456,422\end{array}$ & $\$ 5,617,630$ \\
\hline$\$ 2,723,509$ & $\begin{array}{r}10.50 \% \\
\$ 286,060\end{array}$ & $\begin{array}{r}3500 \% \\
\$ 1,053,349\end{array}$ & $\$ 4,062,918$ \\
\hline$\$ 6,547,320$ & $\begin{array}{r}9.52 \% \\
\$ 623,456\end{array}$ & $\begin{array}{r}35.00 \% \\
\$ 2,509,772\end{array}$ & $\$ 9,680,547$ \\
\hline & & & $\$ 9,700,000$ \\
\hline
\end{tabular}

\begin{tabular}{|ll|l|}
\hline & Remarks \\
\hline Type of Estimate: & Class-5 & \\
Estimator: & A.W. Miller/ S. N. Wasley & \\
Checked By: & & \\
Approved By: & & \\
\hline
\end{tabular}

BEA

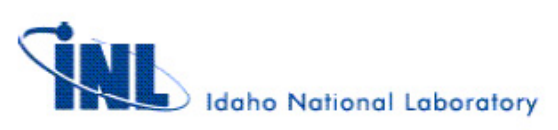

05:11/2012 11:08:00

CostEstimating 


\section{FORMAL COST ESTIMATE SUPPORT DATA RECAPITULATION}

Project Title: $\quad$ RTC Waste Heat Recovery

Estimator: $\quad$ A. W. Miller/S. W. Wasley

Date: May 7,2012

Estimate Type: Class 5

File: $7 \mathrm{~B} 50$

Approved By:

Page 1 of 8

I. PURPOSE: Brief description from the requester of the intent of how the estimate is to be used, i.e., for engineering study, comparative analysis, request for funding, proposal, etc.

The purpose of this estimate is to identify the resources required to complete the scope of work as stated below. It is expected that this estimate will be considered and used by Battelle Energy Alliance, LLC (BEA) in the path forward decision making process for this project.

II. SCOPE OF WORK: Brief statement of the project's objective. Thorough overview and description of the proposed project. Identify work to be accomplished, as well as any specific work to be excluded.

\section{A. Objective:}

The objective of this work is to remove and replace the existing heat recovery piping and insulation system in Building TRA-670. The piping will be removed from the canal storage area, general storage lay-down area, and the Advanced Test Reactor Critical (ATRC) area on the west end of Building TRA-670. This system will be replaced utilizing existing piping racks and penetrations for the new piping system. A new structure will be built to house a heat exchanger and pumps that will tie into the existing secondary cooling water system that will supply water to the heat exchanger system. It is expected that by using the secondary cooling water system from the reactor, the high temperature water will provide energy saving heat through this new piping system, via the existing ventilation system. This work will be performed at the Advanced Test Reactor (ATR) Complex located at Idaho National Laboratory (INL).

\section{B. Included:}

The scope of work required to achieve this objective includes the following:

1. Providing project management support and oversight during the project initiation, design effort, execution phase, and close out.

2. Full time radiological technicians during the subcontracted activities, per construction management.

3. Full time security escorts/guard services for the subcontractors during all activities within TRA-670, per construction management.

4. Quality assurance support.

5. Operations support during outages.

6. Engineering oversight during the execution phase and project support closeout. 
FORMAL COST ESTIMATE SUPPORT DATA RECAPITULATION

-Continued-

Project Title: ATR Waste Heat Recovery

File: 7B50

Page 2 of 8

7. Support to implement new safety analysis reviews (SAR) and unreviewed safety questions (USQ).

8. Environmental assessments will be performed by BEA personnel.

9. A probable risk assessment (PRA) will be performed by BEA personnel to address hazards to the reactor, accident scenarios, etc.

10. Development and/or modifications to existing test procedures, operating procedures, and preventative maintenance procedures to meet the operating requirements of the new heat recovery piping system.

11. Developing and maintaining existing ATR drawings by BEA personnel.

12. Developing operating documents to develop start up/shutdown procedures, monitoring procedures, and system cleanup procedures in case of a glycol spill.

13. Maintenance/operating costs to startup/shutdown, system layup, solution replacement, and system cleaning from corrosion. This estimate includes a 5 year maintenance duration allowance.

14. Training for the BEA crafts to understand and operate the new heat recovery system being installed.

15. General closeout activities including as-built drawings, lessons learned, and training of systems.

16. Subcontracting the execution activities involved with the demolition and installation of the new heat recovery system and the construction of the new heat exchanger building located north of Building TRA-670, includes the following:

a. A general subcontractor to manage and direct lower tier subcontractors performing work in Building TRA-670 and in the new heat exchanger structure.

b. Draining the existing heat recovery system of any existing fluids that may remain in the original system.

c. Removal and disposal of all the existing piping insulation.

d. Demolition and disposal of the existing heat recovery system throughout Building TRA-670.

e. Installation of a new heat recovery piping system and piping insulation on the existing heat recovery piping racks throughout Building TRA-670.

f. Installation of new pressure relief valves, and surge tanks.

g. New coils will be installed in the existing heat recovery systems.

$\mathrm{h}$. Installation of a 50/50 glycol solution in the heat recovery system piping

i. System operation devices for a complete heat recovery operating system.

j. Allowances to tie into the existing secondary piping reactor cooling system located north of Building TRA-670.

k. A new building $60^{\prime} \times 30^{\prime} \times 20^{\prime}$ structure to house the new heat exchanger.

1. Allowances to provide for footings and foundations for the new structure plus a concrete slab floor.

$\mathrm{m}$. New building structure with a roof and lightning protection system.

$\mathrm{n}$. A new heating, ventilating and air conditioning system will be installed in the new structure. 


\title{
FORMAL COST ESTIMATE SUPPORT DATA RECAPITULATION
}

\author{
-Continued-
}

Project Title: ATR Waste Heat Recovery

File: $7 \mathrm{~B} 50$

Page 3 of 8

o. Epoxy paint throughout the new building structure.

p. Allowance for general lighting systems and outlets have been included in the estimate.

q. Alarm and communication system allowance in the new structure.

r. Fire suppression system allowance in the new structure.

s. One new heat exchanger unit will be installed in the new structure.

t. New electrical pumps with motors and piping system will be installed in the new heat exchanger building.

u. Labels will be installed to indentify systems and flow characteristics.

v. Startup and testing of the new system by the subcontractors.

\section{Excluded:}

This scope of work specifically excludes the following:

1. Any upgrades or rebuilding of the existing heat recovery piping supports in Building TRA- 670 .

2. Upgrades to the existing mechanical and electrical systems beyond the identified scope of work.

3. The new heat recovery system will not support any other buildings within the ATR Complex.

4. Project impacts due to encountering asbestos or other hazardous materials that are not explicitly noted in the Building TRA-670 asbestos inspection reports.

5. BEA General and Administrative costs; this project would be funded as indirect.

III. ESTIMATE METHODOLOGY: Overall methodology and rationale of how the estimate was developed, i.e., parametric, forced detail, bottom up, etc. Total dollars/hours and rough order of magnitude (ROM) allocations of the methodologies used to develop the cost estimate.

Because this document will be used to establish the project path forward, a "forced detail" method was used to develop this estimate. This method provides for a greater degree of detail than would be provided utilizing parametric modeling. The activities and resources were developed by the cost estimator and the project team from engineering and project information that provided the level of detail documented in the estimate.

\begin{tabular}{lc}
\hline \multicolumn{1}{c}{ Estimate Methodology } & ROM Percentage (\%) \\
\hline Project Team & 65 \\
Recorded Actuals & 0 \\
Parametric & 0 \\
Vendor Quotes & 0 \\
Other (e.g., RS Means) & 35 \\
\hline TOTAL & 100
\end{tabular}




\title{
FORMAL COST ESTIMATE SUPPORT DATA RECAPITULATION
}

\author{
-Continued-
}

Project Title: ATR Waste Heat Recovery

File: $7 \mathrm{~B} 50$

Page 4 of 8

IV. BASIS OF THE ESTIMATE: Overall explanation of sources for resource quantities, pricing, and schedules.

A. Quantification Basis: The source for the measureable quantities in the estimate that can be used in support of earned value management. Source documents may include drawings, design reports, engineers' notes, and other documentation upon which the estimate is originated.

The requester provided a draft assessment of feasibility, dated March 2012, INL/EXT-12-25004. This preliminary concept provided a write up of the work scope to be executed in Building TRA-670. In addition, a site tour of Building TRA-670 reactor building and other affected areas of the project was used to develop the quantifications. This preliminary work scope information was developed by BEA engineering and was used to establish the activities and quantities for this estimate.

B. Planning Basis: The source for the execution and strategies of the work that can be used to support the project execution plan, identification of long-lead items, acquisition strategy, schedules, market conditions, and other documentation upon which the estimate is originated.

1. Per the requester:

a. This project will be indirect funded.

b. BEA will provide resources for all engineering, project management, and construction management resources.

c. This project will be competitively bid within the local subcontracting community. Local subcontractors familiar with performing work for INL will construct the project.

d. This work will be performed during standard working hours and no premium time will be required for off-shift or weekend work.

e. Work will be able to progress consecutively and will not require delays between work segments.

f. The cost estimate does not consider or address funding or labor resource restrictions. Sufficient funding and labor resources will be available in a manner allowing optimum usage of that funding and resources as estimated.

2. The engineering activities will begin in early FY 2013, with the construction to begin in FY 2014. It is anticipated that the construction will be completed in 9 months.

3. Subcontractor markup rates are based on this estimator's judgment. These rates have been adjusted to reflect the estimated anticipated market conditions.

4. Existing preventative maintenance procedures, operating procedures, and testing procedures will be utilized with minimal modifications to the documents. 


\section{FORMAL COST ESTIMATE SUPPORT DATA RECAPITULATION}

-Continued-

Project Title: ATR Waste Heat Recovery

File: $7 \mathrm{~B} 50$

5. This project will be performed during the normal reactor operation at the TRA-670 facility.

C. Cost Basis: The source for the costing on the estimate that can be used in support of earned value management, funding profiles, and schedule of values. Sources may include published costing references, judgment, actual costs, preliminary quotes and/or other documentation upon which the estimate is originated.

1. INL labor rates, fees, and burdens are based on the current published rates as provided by BEA Planning and Financial Controls.

2. Craft labor rates are based on information provided by the "INL Site Stabilization Agreement." Adders (such as FICA, SUTA, and federal insurances) are based on an interpretation by Cost Estimating.

3. Estimated escalation rates are based on historical indexes from 1962 to today, as published by RS Means. Five-year, ten-year, and lifecycle trends were developed to estimate the most likely rates that have been used in this estimate. Inflationary and deflationary impacts will be addressed in management reserve.

4. Sales tax on materials is based on the current $6 \%$ rate charged by the State of Idaho.

5. Labor factors affecting work being performed within Building TRA-670 have been adjusted. These factors were applied as appropriate in accordance with the project team and estimator's judgment.

6. Estimate includes a full time non-working supervisor/safety representative for the subcontracted work being performed at the ATR complex.

7. Standard published industry references RSMeans and Richardson's Cost Data Online were used to help develop the estimated resources and their productivities and some material costs.

V. ESTIMATE QUALITY ASSURANCE: A listing of all estimate reviews that have taken place and the actions taken from those reviews.

A. A formal review of the cost estimate was held on May 8,2012, with the requester, project team and the cost estimators. This review allowed for the project team to review and comment, in detail, on the perceived scope, basis of estimate, assumptions, project risks, and the resources that make up this cost estimate. Comments from this review have been incorporated into this estimate to reflect a project team consensus of this document.

B. An internal organizational check has been performed on this estimate with the purpose of checking the methodology approach used, discussing the document with the estimator, and ensuring the document has been reviewed and discussed with the requester, reflects a team consensus, has adequately documented the basis, assumptions, and risks to the project, and has mitigated those risks. 


\section{FORMAL COST ESTIMATE SUPPORT DATA RECAPITULATION}

-Continued-

Project Title: ATR Waste Heat Recovery

File: $7 \mathrm{~B} 50$

Page 6 of 8

VI. ASSUMPTIONS: Condition statements accepted or supposed true without proof of demonstration; statements adding clarification to scope. An assumption has a direct impact on total estimated cost.

A. No modification to existing utility systems including code updates, other than the systems where work is being performed to complete the heat recovery project.

B. The pipe removal and installation in Building TRA-670 will be performed during normal reactor operations and also when work is being performed in the canal area.

C. During the existing system removal no asbestos materials will be encountered during the execution of the subcontracted work being performed.

D. Demolition of the existing heat recovery systems is limited to the confines of Building TRA-670.

E. Re-use of existing piping supports without any engineering reanalysis or any physical modifications to the existing heat recovery piping supports in Building TRA-670.

F. This system will not be designed as a safety class system that supports the reactor.

G. No additional confinement penetrations will be required for the heat recovery system installation.

H. The estimate is based on the header supply and return piping to be 8 " in diameter and all the coil branch supply lines and return lines will be 4" in diameter.

I. The new piping system will require insulation and a polyvinyl chloride jacket on the outside of the insulation.

J. There will be sufficient electrical power available to support the new system without any major modifications to electrical equipment existing in Building TRA-670.

$\mathrm{K}$. The existing electrical equipment used in the original heat recovery system will be in good working condition and not require extensive testing and verification to ensure the system is working properly.

L. System tie-ins to the secondary cooling system will be performed during a reactor outage.

M. The new heat exchanger building will be constructed west of TRA-676 (fitness center) located on the north end of Building TRA-670.

$\mathrm{N}$. This estimate includes a redundant pumping system for the secondary cooling side and the glycol loop side of the heat recovery system.

O. No delays will occur while the construction is being performed in the reactor canal area and the ATRC reactor area.

P. Two snorkel lifts will be used to remove the existing piping in the canal area and to reinstall the piping in the canal area. It is assumed the lifts will be able to enter the building through the west end overhead door.

Q. Equipment will be able to access all areas that will require piping removal and installation in the ATRC reactor area.

R. Radiological oversight will be required for all subcontracted activities.

S. No outside work will be performed during adverse weather conditions. 


\title{
FORMAL COST ESTIMATE SUPPORT DATA RECAPITULATION
}

\author{
-Continued-
}

Project Title: ATR Waste Heat Recovery

File: $7 \mathrm{~B} 50$

Page 7 of 8

\section{MANAGEMENT RESERVE (MR) GUIDELINE IMPLEMENTATION:}

Management Reserve Methodologies: Explanation of methodology used in determining overall management reserve. Identify any specific drivers or items of concern and any inherent risks typical with this type of environment. Inflationary and deflationary impacts are addressed in this section.

No formal risk review was performed for this project. A blanket management reserve allowance of $35 \%$ was applied to this cost estimate. This allowance was concurred with by the cost estimators and the project team as generally reflective of the risks and assumptions stated in this document, and as appropriate for the purpose of this cost estimate.

A. Threats: Uncertain events that are potentially negative or reduce the probability that the desired outcome will happen.

1. No detailed design exists for this project. The estimated costs were based on the cost estimator's perceived idea as to the design requirements and project scope that will be required. Completion of the design may increase the costs due to requirements or needs not identified in the scope of this estimate.

2. Preciseness in the forced detail take-offs leaves little room if crews are unable to meet the estimated production rates. Factors could include, but are not limited to, changes to Integrated Safety Management requirements, equipment breakdowns, resource impacts, and/or availability.

3. Possible glycol solution leaking into the secondary reactor cooling system through a breach in the heat exchanger, this could cause cross contamination in the systems.

4. Flooding in the TRA-670 reactor building due to a break in the system or a manufacturer's defect in the materials that will be installed.

5. More than normal adverse weather (cold, snow, rain, and wind) would cause losses in productivity or even stop the work. This loss in productivity or stoppage would still require for the operating contractor's forces to be compensated.

6. Impacts due to ongoing ATR operation activities could delay the project.

7. If the existing pipe hangers do not meet the current seismic requirements an evaluation and installation of new pipe hangers could be required.

B. Opportunities: Uncertain events that could improve the results or improve the probability that the desired outcome will happen.

1. Well-planned-out work activities and scheduling by the subcontractors could result in increased production, thus producing lower bids and operating contractor oversight costs than what have been estimated.

2. Optimization of the design could reduce the utility runs, thus reducing the costs associated with those activities. 
FORMAL COST ESTIMATE SUPPORT DATA RECAPITULATION

-Continued-

Project Title: ATR Waste Heat Recovery

File: $7 \mathrm{~B} 50$

Page 8 of 8

3. In the event standard excavation activities could be performed in lieu of vacuum excavation.

C. Accepted Risks: Activities with a greater than 50\% and less than $100 \%$ probability of occurrence has been accepted as part of this scope of work.

NA

D. Excluded Risks: Risks that have been identified and have a probability of occurrence but are specifically excluded from this estimate.

NA

Note: Management reserve does not increase the overall accuracy of the estimate; it does, however, reduce the level of risk associated with the estimate. Management reserve is intended to cover the inadequacies in the complete project scope definition, estimating methods, and estimating data. Management reserve specifically excludes changes in project scope, unexpected work stoppages, (e.g., strikes, disasters, and earthquakes) and excessive and/or unexpected inflation or currency fluctuations. This estimate does not contain any contingencies and has not been evaluated to include any contingencies and has not been evaluated to include any of the risks that pertain to Department of Energy.

\section{OTHER COMMENTS/CONCERNS SPECIFIC TO THE ESTIMATE:}

None. 


\section{Project Name: RTC Waste Heat Recovery}

Project Location: ATR

Number: 7850

\begin{tabular}{|c|c|c|}
\hline Level & Group & Description \\
\hline$\overline{1.0}$ & & Project Management (PM) \\
\hline 1.1 & OPC & ....Project Management Initiation \\
\hline 1.2 & & .... Project Management (PM) Support during Design Phase \\
\hline 1.2.1 & & $\begin{array}{l}\text {....... Provide PM Oversight - Level of Effort (LOE) } \\
\text { during Design Phase }\end{array}$ \\
\hline 1.2.2 & & $\begin{array}{l}\text {........ Maintain Project Files - LOE during Design } \\
\text { Phase }\end{array}$ \\
\hline 1.2.3 & & $\begin{array}{l}\text {.......Provide cost and Schedule Support during } \\
\text { Design Phase }\end{array}$ \\
\hline 1.3 & & .... Project Management (PM) Support during Execution \\
\hline 1.3.1 & & $\begin{array}{l}\text {........Provide PM Oversight - Level of Effort (LOE) } \\
\text { during Execution }\end{array}$ \\
\hline 1.3.2 & & ........ Maintain Project Files - LOE during Execution \\
\hline 1.3.3 & & $\begin{array}{l}\text {........Provide cost and Schedule Support during } \\
\text { Execution }\end{array}$ \\
\hline 1.4 & & .... Quality Assurance and Quality Control Support \\
\hline 1.4.1 & OPC & $\begin{array}{l}\text {.......Provide Quality Inspections During } \\
\text { construction - First Line }\end{array}$ \\
\hline 1.4.2 & OPC & ........ Quality Assurance Oversight \\
\hline 2.0 & & Construction Management (CM) \\
\hline 2.1 & & $\begin{array}{l}\text {....Provide CM Oversight/Support during Execution - Level } \\
\text { of Effort (LOE) }\end{array}$ \\
\hline 2.2 & OPC & .... BEA - Provide CM Supervision - OPC \\
\hline 2.3 & OPC & „...P Provide Safety Oversight/Support during Execution - \\
\hline 2.4 & OPC & $\begin{array}{l}\text {.... Provide Industrial Hygiene Oversight/Support during } \\
\text { Execution - LOE }\end{array}$ \\
\hline 2.5 & & $\begin{array}{l}\text {.... Perform Subsurface Investigations Prior to Excav'ns, } \\
\text { Floor and Wall Penetrations }\end{array}$ \\
\hline
\end{tabular}

\section{Project Summary Report}

\begin{tabular}{|c|c|c|c|c|}
\hline \multirow[b]{2}{*}{$\begin{array}{l}\text { Estimate } \\
\text { Subtotal } \\
\$ 402,470\end{array}$} & \multirow[b]{2}{*}{$\frac{\text { Escalation }}{\$ 30,484}$} & \multicolumn{3}{|c|}{$\begin{array}{ll}\text { Client: } & \text { C.P. Ischay } \\
\text { Prepared By: } & \text { A.W. Miller/S. N. Wasley } \\
\text { Estimate Type: } & \text { Class-5 }\end{array}$} \\
\hline & & $\begin{array}{l}\text { Management } \\
\text { Reserve MR } \\
\$ 151,534\end{array}$ & $\begin{array}{l}\frac{\mathrm{MR}}{\%} \\
35.00 \%\end{array}$ & $\frac{\text { TOTAL }}{\$ 584,488}$ \\
\hline$\$ 53,867$ & $\$ 2,995$ & $\$ 19,902$ & $35.00 \%$ & $\$ 76,764$ \\
\hline$\$ 130,099$ & $\$ 7,233$ & $\$ 48,066$ & $35.00 \%$ & $\$ 185,398$ \\
\hline$\$ 69,087$ & $\$ 3,841$ & $\$ 25,525$ & $35.00 \%$ & $\$ 98,453$ \\
\hline$\$ 10,327$ & $\$ 574$ & $\$ 3,815$ & $35.00 \%$ & $\$ 14,717$ \\
\hline$\$ 50,684$ & $\$ 2,818$ & $\$ 18,726$ & $35.00 \%$ & $\$ 72,228$ \\
\hline$\$ 158,961$ & $\$ 14,736$ & $\$ 60,794$ & $35.00 \%$ & $\$ 234,491$ \\
\hline$\$ 91,700$ & $\$ 8,501$ & $\$ 35,070$ & $35.00 \%$ & $\$ 135,270$ \\
\hline$\$ 13,770$ & $\$ 1,276$ & $\$ 5,266$ & $35.00 \%$ & $\$ 20,312$ \\
\hline$\$ 53,492$ & $\$ 4,959$ & $\$ 20,458$ & $\mathbf{3 5 . 0 0 \%}$ & $\$ 78,908$ \\
\hline$\$ 59,543$ & $\$ 5,520$ & $\$ 22,772$ & $35.00 \%$ & $\$ 87,835$ \\
\hline$\$ 50,650$ & $\$ 4,695$ & $\$ 19,371$ & $35.00 \%$ & $\$ 74,716$ \\
\hline$\$ 8,894$ & $\$ 824$ & $\$ 3,401$ & $35.00 \%$ & $\$ 13,119$ \\
\hline$\$ 313,848$ & $\$ 29,094$ & $\$ 120,029$ & $35.00 \%$ & $\$ 462,971$ \\
\hline$\$ 122,397$ & $\$ 11,346$ & $\$ 46,810$ & $35.00 \%$ & $\$ 180,554$ \\
\hline$\$ 14,611$ & $\$ 1,354$ & $\$ 5,588$ & $35.00 \%$ & $\$ 21,554$ \\
\hline$\$ 110,390$ & $\$ 10,233$ & $\$ 42,218$ & $35.00 \%$ & $\$ 162,842$ \\
\hline$\$ 50,864$ & $\$ 4,715$ & $\$ 19,453$ & $35.00 \%$ & $\$ 75,032$ \\
\hline$\$ 11,475$ & $\$ 1,064$ & $\$ 4,389$ & $35.00 \%$ & $\$ 16,928$ \\
\hline
\end{tabular}

\section{BEA}




\begin{tabular}{|c|c|c|}
\hline Level & Group & Description \\
\hline 2.6 & & .... CM Support During Design and Closeout \\
\hline 3.0 & & Engineering/Design \\
\hline 3.1 & & $\ldots$. BEA Engineering \& Design Phase \\
\hline 3.1.1 & OPC & ....... BEA Engineering \& Design Conceptual Phase \\
\hline 3.1.2 & & ....... BEA Engineering \& Design Preliminary Phase \\
\hline 3.1.3 & & ....... BEA Engineering \& Design Final Phase \\
\hline 4.0 & OPC & Design Plans, Reports \& Documents \\
\hline 4.1 & OPC & .... Environmental Documents (NEPA Checklist) \\
\hline 4.2 & OPC & .... Hazards Documents \\
\hline 4.2.1 & OPC & ........ Prepare Fire Hazards Analysis Report \\
\hline 4.3 & OPC & .... Other Documents \\
\hline 4.3.1 & OPC & ....... Prepare Davis Bacon Determination Documents \\
\hline 4.4 & OPC & .... Prepare Unreviewed Safety Questions (USQs) \\
\hline 4.5 & OPC & .... Probablistic Risk Assessment (PRA) \\
\hline 4.5.1 & OPC & $\begin{array}{l}\text {........ Probabilistic Risk Assessment (PRA) } \\
\text { Preliminary }\end{array}$ \\
\hline 4.5.2 & OPC & ........ Probabilistic Risk Assessment (PRA) Final \\
\hline 5.0 & & Execution Phase \\
\hline 5.1 & & .... Subcontract Construction \\
\hline 5.1.1 & & ......... General Conditions \\
\hline 5.1.1.1 & & ........... General Contractor \\
\hline 5.1.1.2 & & $\ldots \ldots \ldots$ Piping Contractor \\
\hline 5.1 .1 .3 & & .......... Electrical Contractor \\
\hline 5.1.2 & & $\begin{array}{l}\text {........ Building TRA-670 Heat Recovery } \\
\text { Demolition/Installation }\end{array}$ \\
\hline 5.1 .2 .1 & & ...... Waste Heat Recovery Piping Demolition \\
\hline
\end{tabular}

Project Name: RTC Waste Heat Recovery

Project Location: ATR
Estimate Number:7B50

Project Summary Report

\begin{tabular}{|c|c|c|c|c|}
\hline $\begin{array}{r}\text { Estimate } \\
\text { Subtotal } \\
\$ 4,109\end{array}$ & $\frac{\text { Escalation }}{\$ 381}$ & $\begin{array}{c}\text { Management } \\
\text { Reserve MR } \\
\$ 1,572\end{array}$ & $\begin{array}{l}\text { MR } \\
\frac{\%}{35.00} \%\end{array}$ & $\frac{\text { TOTAL }}{\$ 6,062}$ \\
\hline$\$ 495,077$ & $\$ 27,526$ & $\$ 182,911$ & $35.00 \%$ & $\$ 705,514$ \\
\hline$\$ 495,077$ & $\$ 27,526$ & $\$ 182,911$ & $35.00 \%$ & $\$ 705,514$ \\
\hline$\$ 165,026$ & $\$ 9,175$ & $\$ 60,970$ & $35.00 \%$ & $\$ 235,171$ \\
\hline$\$ 126,829$ & $\$ 7,052$ & $\$ 46,858$ & $35.00 \%$ & $\$ 180,739$ \\
\hline$\$ 203,222$ & $\$ 11,299$ & $\$ 75,082$ & $35.00 \%$ & $\$ 289,604$ \\
\hline$\$ 197,699$ & $\$ 10,992$ & $\$ 73,042$ & $35.00 \%$ & $\$ 281,733$ \\
\hline$\$ 4,898$ & $\$ 272$ & $\$ 1,809$ & $35.00 \%$ & $\$ 6,979$ \\
\hline$\$ 22,568$ & $\$ 1,255$ & $\$ 8,338$ & $35.00 \%$ & $\$ 32,161$ \\
\hline$\$ 22,568$ & $\$ 1,255$ & $\$ 8,338$ & $35.00 \%$ & $\$ 32,161$ \\
\hline$\$ 11,431$ & $\$ 636$ & $\$ 4,223$ & $35.00 \%$ & $\$ 16,290$ \\
\hline$\$ 11,431$ & $\$ 636$ & $\$ 4,223$ & $35.00 \%$ & $\$ 16,290$ \\
\hline$\$ 85,889$ & $\$ 4,775$ & $\$ 31,732$ & $35.00 \%$ & $\$ 122,397$ \\
\hline$\$ 72,913$ & $\$ 4,054$ & $\$ 26,939$ & $35.00 \%$ & $\$ 103,906$ \\
\hline$\$ 29,941$ & $\$ 1,665$ & $\$ 11,062$ & $35.00 \%$ & $\$ 42,667$ \\
\hline$\$ 42,973$ & $\$ 2,389$ & $\$ 15,877$ & $35.00 \%$ & $\$ 61,239$ \\
\hline$\$ 3,602,929$ & $\$ 333,992$ & $\$ 1,377,922$ & $35.00 \%$ & $\$ 5,314,843$ \\
\hline$\$ 3,066,718$ & $\$ 284,285$ & $\$ 1,172,851$ & $35.00 \%$ & $\$ 4,523,854$ \\
\hline$\$ 319,025$ & $\$ 29,574$ & $\$ 122,009$ & $35.00 \%$ & $\$ 470,608$ \\
\hline$\$ 144,214$ & $\$ 13,369$ & $\$ 55,154$ & $35.00 \%$ & $\$ 212,737$ \\
\hline$\$ 99,904$ & $\$ 9,261$ & $\$ 38,208$ & $35.00 \%$ & $\$ 147,373$ \\
\hline$\$ 74,906$ & $\$ 6,944$ & $\$ 28,648$ & $35.00 \%$ & $\$ 110,498$ \\
\hline$\$ 1,263,281$ & $\$ 117,106$ & $\$ 483,135$ & $35.00 \%$ & $\$ 1,863,522$ \\
\hline$\$ 252,871$ & $\$ 23,441$ & $\$ 96,709$ & $35.00 \%$ & $\$ 373,021$ \\
\hline
\end{tabular}

BEA 
Project Name: RTC Waste Heat Recovery

Project Location: ATR

Estimate Number: 7B50

$\frac{\text { Level }}{5.1 .21 .1}$

Group

5.1.2.1.2

Demoltion

\section{Project Summary Report}

5.1.2.1.3

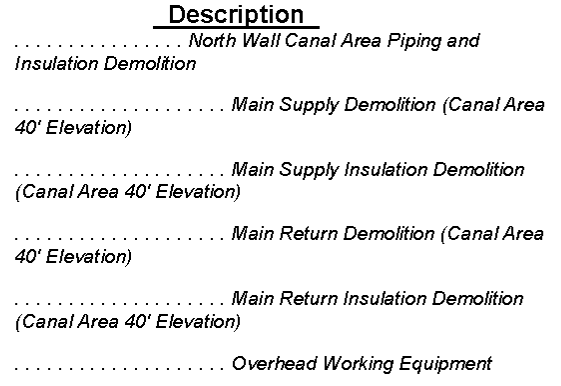

Mezzanine Area 40. Elevation) Supply Demollition (Canal

..................in Supply Insulation Demolition

Main Return Demolition (Canal

Mezzanine Area 40. Elevation)

................. Main Return Insulation Demolition (Canal Mezzanine Area 40'Elevation

................ Overhead Working Equipment

Demolition

Area $30^{\prime}$ Elevation

Main Supply Demolition (West Canal

.................... Main Supply Insulation Demolition

................. Main Return Demolltion (West Cana

(W.................. Main Return Insulation Demolition st Canal Area 30' Elevation)

Overhead Working Equipment

\begin{tabular}{|c|c|c|c|c|}
\hline \multirow[b]{2}{*}{$\begin{array}{l}\text { Estimate } \\
\text { Subtotal } \\
\$ 62,569\end{array}$} & \multirow[b]{2}{*}{$\frac{\text { Escalation }}{\$ 5,800}$} & \multicolumn{3}{|c|}{$\begin{array}{ll}\text { Client: } & \text { C.P. Ischay } \\
\text { Prepared By: } & \text { A.W. Miller/S. N. Wasley } \\
\text { Estimate Type: } & \text { Class-5 }\end{array}$} \\
\hline & & $\begin{array}{r}\text { Management } \\
\text { Reserve MR } \\
\$ 23,929\end{array}$ & $\begin{array}{l}\text { MR } \\
\frac{\%}{35.00 \%}\end{array}$ & $\frac{\text { TOTAL }}{\$ 92,299}$ \\
\hline$\$ 7,312$ & $\$ 678$ & $\$ 2,796$ & $35.00 \%$ & $\$ 10,786$ \\
\hline$\$ 15,648$ & $\$ 1,451$ & $\$ 5,985$ & $35.00 \%$ & $\$ 23,084$ \\
\hline$\$ 7,312$ & $\$ 678$ & $\$ 2,796$ & $35.00 \%$ & $\$ 10,786$ \\
\hline$\$ 15,648$ & $\$ 1,451$ & $\$ 5,985$ & $35.00 \%$ & $\$ 23,084$ \\
\hline$\$ 16,648$ & $\$ 1,543$ & $\$ 6,367$ & $35.00 \%$ & $\$ 24,558$ \\
\hline$\$ 24,075$ & $\$ 2,232$ & $\$ 9,207$ & $35.00 \%$ & $\$ 35,515$ \\
\hline$\$ 2,559$ & $\$ 237$ & $\$ 979$ & $35.00 \%$ & $\$ 3,775$ \\
\hline$\$ 5,477$ & $\$ 508$ & $\$ 2,095$ & $35.00 \%$ & $\$ 8,079$ \\
\hline$\$ 2,559$ & $\$ 237$ & $\$ 979$ & $35.00 \%$ & $\$ 3,775$ \\
\hline$\$ 5,477$ & $\$ 508$ & $\$ 2,095$ & $35.00 \%$ & $\$ 8,079$ \\
\hline$\$ 8,003$ & $\$ 742$ & $\$ 3,061$ & $35.00 \%$ & $\$ 11,805$ \\
\hline$\$ 24,075$ & $\$ 2,232$ & $\$ 9,207$ & $35.00 \%$ & $\$ 35,515$ \\
\hline$\$ 2,559$ & $\$ 237$ & $\$ 979$ & $35.00 \%$ & $\$ 3,775$ \\
\hline$\$ 5,477$ & $\$ 508$ & $\$ 2,095$ & $35.00 \%$ & $\$ 8,079$ \\
\hline$\$ 2,559$ & $\$ 237$ & $\$ 979$ & $35.00 \%$ & $\$ 3,775$ \\
\hline$\$ 5,477$ & $\$ 508$ & $\$ 2,095$ & $35.00 \%$ & $\$ 8,079$ \\
\hline$\$ 8,003$ & $\$ 742$ & $\$ 3,061$ & $35.00 \%$ & $\$ 11,805$ \\
\hline
\end{tabular}

BEA 
Project Name: RTC Waste Heat Recovery

Project Location: ATR

\begin{tabular}{|c|c|c|}
\hline$\frac{\text { Level }}{5.1 .2 .1 .4}$ & Group & 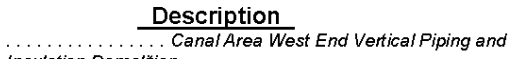 \\
\hline & & \\
\hline & & 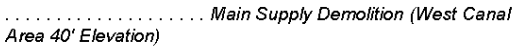 \\
\hline & & $\begin{array}{l}\text { (West Canal Area } 40^{\circ} \text { Elevation) } \\
\text { (Wepply Insulation Demolltion }\end{array}$ \\
\hline & & $\begin{array}{l}\ldots \ldots \ldots \ldots \ldots . \ldots \text { Main Return Demolition (West Canal } \\
\text { Area } 40^{\prime} \text { Elevation) }\end{array}$ \\
\hline & & $\ldots \ldots \ldots \ldots \ldots \ldots$ Main Return Insulation Demolltion \\
\hline & & (West Canal Area $40^{\prime}$ Elevation) \\
\hline & & ................ Overhead Working Equipment \\
\hline 5.1.2.1.5 & & $\begin{array}{l}\text { I................... West End Laydown Area Metal Piping and } \\
\text { insulation Demoltion }\end{array}$ \\
\hline & & Area West Wail $20^{\prime}$ Elevation) \\
\hline & & $\begin{array}{l}\text { (Laydown Area West Wall 20. Main Supply Insulation Demolition } \\
\text { (Lation) }\end{array}$ \\
\hline & & 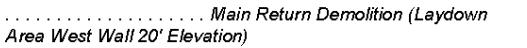 \\
\hline & & 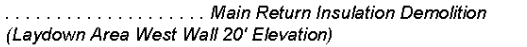 \\
\hline & & ................... Overhead Working Equipment \\
\hline 5.1.2.1.6 & & $\begin{array}{l}\ldots \ldots \ldots \ldots . \ldots \text { North End Laydown Area Metal Piping and } \\
\text { insulation Demoltition }\end{array}$ \\
\hline & & 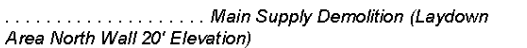 \\
\hline & & $\begin{array}{l}\text { Main Supply ynsulation Demolition } \\
\text { (Laydown Area North Wall } 20^{\circ} \text { Elevation) }\end{array}$ \\
\hline & & Area North Wall 20... Main Return Demolition (Laydown \\
\hline & & $\begin{array}{l}\text { (Laydown Area North Wall } 20^{\circ} \text { Elevation) } \\
\text { (Lation Demolition }\end{array}$ \\
\hline & & . Overhead Working Equipment \\
\hline
\end{tabular}

\section{Project Summary Report}

\begin{tabular}{|c|c|c|c|c|}
\hline & & $\begin{array}{ll}\text { Client: } & \text { C.P. } \\
\text { Prepared By: } & \text { A.W } \\
\text { Estimate Type: } & \text { Clas }\end{array}$ & $\begin{array}{l}\text { schay } \\
\text { willer/s. } \\
5\end{array}$ & \\
\hline $\begin{array}{l}\text { timate } \\
\text { tbtotal } \\
\$ \$ 14,891\end{array}$ & $\frac{\text { Escalation }}{\$ 1,380}$ & $\begin{array}{c}\text { Management } \\
\text { Reserve MR } \\
\$ 5,695\end{array}$ & $\begin{array}{l}\text { MR } \\
\frac{\%}{35.00 \%}\end{array}$ & $\frac{\text { TOTAL }}{\$ 21,966}$ \\
\hline$\$ 1,097$ & $\$ 102$ & $\$ 419$ & $35.00 \%$ & $\$ 1,618$ \\
\hline$\$ 2,347$ & $\$ 218$ & $\$ 898$ & $35.00 \%$ & $\$ 3,463$ \\
\hline$\$ 1,097$ & $\$ 102$ & $\$ 419$ & $35.00 \%$ & $\$ 1,618$ \\
\hline$\$ 2,347$ & $\$ 218$ & $\$ 898$ & $35.00 \%$ & $\$ 3,463$ \\
\hline$\$ 8,003$ & $\$ 742$ & $\$ 3,061$ & $35.00 \%$ & $\$ 11,805$ \\
\hline$\$ 24,478$ & $\$ 2,269$ & $\$ 9,361$ & $35.00 \%$ & $\$ 36,108$ \\
\hline$\$ 2,760$ & $\$ 256$ & $\$ 1,056$ & $35.00 \%$ & $\$ 4,072$ \\
\hline$\$ 5,477$ & $\$ 508$ & $\$ 2,095$ & $35.00 \%$ & $\$ 8,079$ \\
\hline$\$ 2,760$ & $\$ 256$ & $\$ 1,056$ & $35.00 \%$ & $\$ 4,072$ \\
\hline$\$ 5,477$ & $\$ 508$ & $\$ 2,095$ & $35.00 \%$ & $\$ 8,079$ \\
\hline$\$ 8,003$ & $\$ 742$ & $\$ 3,061$ & $35.00 \%$ & $\$ 11,805$ \\
\hline$\$ 26,831$ & $\$ 2,487$ & $\$ 10,261$ & $35.00 \%$ & $\$ 39,580$ \\
\hline$\$ 3,155$ & $\$ 292$ & $\$ 1,207$ & $35.00 \%$ & $\$ 4,654$ \\
\hline$\$ 6.259$ & $\$ 580$ & $\$ 2,394$ & $35.00 \%$ & $\$ 9.233$ \\
\hline$\$ 3,155$ & $\$ 292$ & $\$ 1,207$ & $35.00 \%$ & $\$ 4,654$ \\
\hline$\$ 6,259$ & $\$ 580$ & $\$ 2,394$ & $35.00 \%$ & $\$ 9,233$ \\
\hline$\$ 8,003$ & $\$ 742$ & $\$ 3,061$ & $35.00 \%$ & $\$ 11,805$ \\
\hline
\end{tabular}

\section{BEA}


Project Name: RTC Waste Heat Recovery

Project Location: ATR

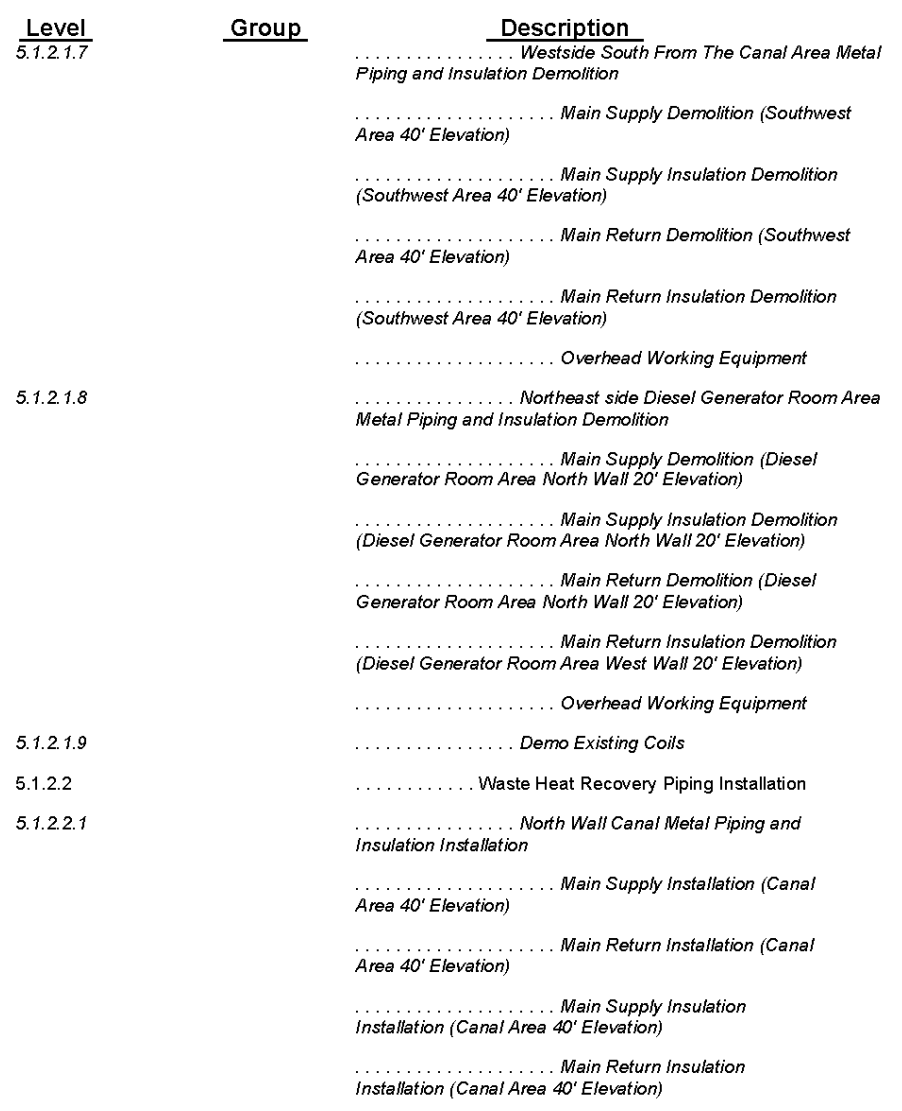

BEA

05/11/2012 11:08:29

\section{Project Summary Report}

\begin{tabular}{|c|c|c|c|c|}
\hline \multirow[b]{2}{*}{$\begin{array}{l}\text { stimate } \\
\frac{\text { ubtotal }}{\$ 48,955}\end{array}$} & \multirow[b]{2}{*}{$\frac{\text { Escalation }}{\$ 4,538}$} & \multicolumn{3}{|c|}{$\begin{array}{ll}\text { Client: } & \text { C.P. Ischay } \\
\text { Prepared By: } & \text { A.W. Miller/S. N. Wasley } \\
\text { Estimate Type: Class-5 }\end{array}$} \\
\hline & & $\begin{array}{l}\text { Management } \\
\text { Reserve MR } \\
\$ 18,723\end{array}$ & $\begin{array}{l}\frac{\mathrm{MR}}{\%} \\
\frac{35.00 \%}{2}\end{array}$ & $\frac{\text { TOTAL }}{\$ 72,216}$ \\
\hline$\$ 5,521$ & $\$ 512$ & $\$ 2,111$ & $35.00 \%$ & $\$ 8,144$ \\
\hline$\$ 10,954$ & $\$ 1,015$ & $\$ 4,189$ & $35.00 \%$ & $\$ 16,159$ \\
\hline$\$ 5,521$ & $\$ 512$ & $\$ 2,111$ & $35.00 \%$ & $\$ 8,144$ \\
\hline$\$ 10,954$ & $\$ 1,015$ & $\$ 4,189$ & $35.00 \%$ & $\$ 16,159$ \\
\hline$\$ 16,006$ & $\$ 1,484$ & $\$ 6,121$ & $35.00 \%$ & $\$ 23,611$ \\
\hline$\$ 19,771$ & $\$ 1,833$ & $\$ 7,561$ & $35.00 \%$ & $\$ 29,164$ \\
\hline$\$ 1,972$ & $\$ 183$ & $\$ 754$ & $35.00 \%$ & $\$ 2,909$ \\
\hline$\$ 3,912$ & $\$ 363$ & $\$ 1,496$ & $35.00 \%$ & $\$ 5,771$ \\
\hline$\$ 1,972$ & $\$ 183$ & $\$ 754$ & $35.00 \%$ & $\$ 2,909$ \\
\hline$\$ 3,912$ & $\$ 363$ & $\$ 1,496$ & $35.00 \%$ & $\$ 5,771$ \\
\hline$\$ 8,003$ & $\$ 742$ & $\$ 3,061$ & $35.00 \%$ & $\$ 11,805$ \\
\hline$\$ 7,225$ & $\$ 670$ & $\$ 2,763$ & $35.00 \%$ & $\$ 10,659$ \\
\hline $1,010,410$ & $\$ 93,665$ & $\$ 386,426$ & $35.00 \%$ & $\$ 1,490,501$ \\
\hline$\$ 155,560$ & $\$ 14,420$ & $\$ 59,493$ & $35.00 \%$ & $\$ 229,474$ \\
\hline$\$ 55,214$ & $\$ 5,118$ & $\$ 21,116$ & $35.00 \%$ & $\$ 81,448$ \\
\hline$\$ 55,214$ & $\$ 5,118$ & $\$ 21,116$ & $35.00 \%$ & $\$ 81,448$ \\
\hline$\$ 10,080$ & $\$ 934$ & $\$ 3,855$ & $35.00 \%$ & $\$ 14,870$ \\
\hline$\$ 10,080$ & $\$ 934$ & $\$ 3,855$ & $35.00 \%$ & $\$ 14,870$ \\
\hline
\end{tabular}




\begin{tabular}{|c|c|c|}
\hline \multicolumn{3}{|c|}{$\begin{array}{l}\text { Project Location: ATR } \\
\text { Estimate Number: } 7850\end{array}$} \\
\hline Level & Group & $\frac{\text { Description }}{\ldots \ldots \text { Overhead Working Equipment }}$ \\
\hline \multirow[t]{6}{*}{ 5.1.2.2.2 } & & $\begin{array}{l}\text { Znsulation instalfion } \\
\text { ineal Mezzanine Metal Piping and }\end{array}$ \\
\hline & & Area $40^{\prime}$ Elevetion) \\
\hline & & $\begin{array}{l}\text { Area } 40 \text { 'Elevation) } \\
\text { A Main Return Installation (Canal }\end{array}$ \\
\hline & & $\begin{array}{l}\ldots \ldots \ldots \ldots \ldots \ldots \text { Main Supply insulation } \\
\text { installation (Canal Area 40 40 Elevation) }\end{array}$ \\
\hline & & $\begin{array}{l}\ldots \ldots \ldots \ldots \ldots \text { Main Return insulation } \\
\text { installation (Canal Area 40 Elevation) }\end{array}$ \\
\hline & & ......... Overhead Working Equipment \\
\hline \multirow[t]{6}{*}{ 5.1.2.2.3 } & & installation \\
\hline & & $\begin{array}{l}\ldots \ldots \ldots \ldots \ldots \text { Main Supply installation (West } \\
\text { Canal Area } 30^{\circ} \text { Elevation) }\end{array}$ \\
\hline & & $\begin{array}{l}\ldots \ldots \ldots \ldots \ldots \ldots \text { Main Return installation (West } \\
\text { Canal Area } 30^{\circ} \text { Elevation) }\end{array}$ \\
\hline & & $\begin{array}{l}\text {....................... Main Supply Insulation } \\
\text { installation (West Canai Area } 30^{\prime} \text { Elevation) }\end{array}$ \\
\hline & & $\begin{array}{l}\ldots \ldots \ldots \ldots \ldots \ldots \text { Main Return Insulation } \\
\text { installation (West Canai Area } 30^{\prime} \text { Elevation) }\end{array}$ \\
\hline & & .............. Overhead Working Equipment \\
\hline \multirow[t]{6}{*}{ 5.1.2.2.4 } & & $\begin{array}{l}\ldots \ldots \ldots \ldots \ldots \text { Canal Area West End Vertical Piping and } \\
\text { insulation Installation }\end{array}$ \\
\hline & & Canal Area $40^{\circ}$ Elevation) \\
\hline & & $\begin{array}{l}\ldots \ldots \ldots \ldots \ldots \text { Main Return Installation (West } \\
\text { Canal Area } 40^{\circ} \text { Elevation) }\end{array}$ \\
\hline & & 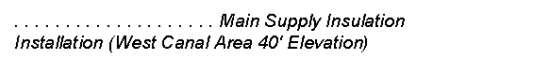 \\
\hline & & $\begin{array}{l}\ldots \ldots \ldots \ldots \ldots \text { Main Return insulation } \\
\text { installation (West Canal Area 40' Elevation) }\end{array}$ \\
\hline & & $\ldots \ldots \ldots \ldots \ldots \ldots$ Overhead Working Equipment \\
\hline
\end{tabular}

BEA

05/11/2012 11:08:29
Project Name: RTC Waste Heat Recovery

Project Location: ATR

\section{Project Summary Report}

\section{.}

Estimate

Subtotal

$\$ 59,847$

$\$ 17,465$

$\$ 17,465$

$\$ 4,134$

$\$ 4,134$

$\$ 16,648$

$\$ 53,442$

$\$ 19,303$

$\$ 19,303$

$\$ 3,417$

$\$ 3,417$

$\$ 8,003$

$\$ 26,502$

$\$ 7,734$

$\$ 7,734$

$\$ 1,516$

$\$ 1,516$

$\$ 8,003$
Client: C.P. Ischay

Prepared By: A.W. Miller/S. N. Wasley
Estimate Type: Class-5

\begin{tabular}{|c|c|c|}
\hline $\begin{array}{c}\text { Management } \\
\text { Reserve MR } \\
\$ 9,550\end{array}$ & $\begin{array}{l}\text { MR } \\
\frac{\%}{35.00 \%}\end{array}$ & $\frac{\text { TOTAL }}{\$ 36,837}$ \\
\hline$\$ 22,888$ & $35.00 \%$ & $\$ 88,284$ \\
\hline$\$ 6,680$ & $35.00 \%$ & $\$ 25,764$ \\
\hline$\$ 6,680$ & $35.00 \%$ & $\$ 25,764$ \\
\hline$\$ 1,581$ & $35.00 \%$ & $\$ 6,099$ \\
\hline$\$ 1,581$ & $35.00 \%$ & $\$ 6,099$ \\
\hline$\$ 6,367$ & $35.00 \%$ & $\$ 24,558$ \\
\hline$\$ 20,439$ & $35.00 \%$ & $\$ 78,835$ \\
\hline$\$ 7,382$ & $35.00 \%$ & $\$ 28,474$ \\
\hline$\$ 7,382$ & $35.00 \%$ & $\$ 28,474$ \\
\hline$\$ 1,307$ & $35.00 \%$ & $\$ 5,040$ \\
\hline$\$ 1,307$ & $35.00 \%$ & $\$ 5,040$ \\
\hline$\$ 3,061$ & $35.00 \%$ & $\$ 11,805$ \\
\hline$\$ 10,135$ & $35.00 \%$ & $\$ 39,094$ \\
\hline$\$ 2,958$ & $35.00 \%$ & $\$ 11,408$ \\
\hline$\$ 2,958$ & $35.00 \%$ & $\$ 11,408$ \\
\hline$\$ 580$ & $35.00 \%$ & $\$ 2,236$ \\
\hline$\$ 580$ & $35.00 \%$ & $\$ 2,236$ \\
\hline$\$ 3,061$ & $35.00 \%$ & $\$ 11,805$ \\
\hline
\end{tabular}


Project Name: RTC Waste Heat Recovery

Project Location: ATR

\begin{tabular}{|c|c|c|}
\hline$\frac{\text { Level }}{5.1 .2 .2 .5}$ & Group & $\frac{\text { Description }}{\text { West End Laydown Area Metal Piping and }}$ \\
\hline & & Insulation installation \\
\hline & & Area 30'Elevation) \\
\hline & & $\begin{array}{l}\ldots \ldots \ldots \ldots \ldots \ldots \text { Main Return Installation (Canal } \\
\text { Area } 30^{\circ} \text { Elevation) }\end{array}$ \\
\hline & & $\begin{array}{l}\ldots \ldots \ldots \ldots \ldots \ldots \text { Main Supply insulation } \\
\text { installation (Canal Area 30' Elevation) }\end{array}$ \\
\hline & & .....................Main Return Insulation \\
\hline & & . O Overhead Working Equipment \\
\hline 5.1.2.2.6 & & $\begin{array}{l}\ldots \ldots \ldots . \ldots . \ldots \text { North End Laydown Area Metal Piping and } \\
\text { insulation Installation }\end{array}$ \\
\hline & & Area 20 Elevation).... Main Supply installation (Canal \\
\hline & & .. Main Return Installation (Canal \\
\hline & & ................... Main Supply fnsulation \\
\hline & & Installation (Cana/Area 20 "Elevation) \\
\hline & & 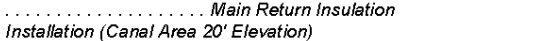 \\
\hline & & ............... Overhead Working Equipment \\
\hline 5.1.2.2.7 & & $\begin{array}{l}\ldots \ldots \ldots \ldots \text { Westside South From The Canal Area Metal } \\
\text { Piping and insulation installation }\end{array}$ \\
\hline & & ... Main Supply Installation (Canal \\
\hline & & Main Return Installation (Canal \\
\hline & & $\begin{array}{l}\ldots \ldots \ldots \ldots \text { Main Supply insulation } \\
\text { installation (Canal Area 40 Elevation) }\end{array}$ \\
\hline & & 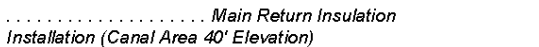 \\
\hline & & Overhead Working Equipment \\
\hline
\end{tabular}

\section{Project Summary Report}

\begin{tabular}{|c|c|c|c|c|}
\hline & & $\begin{array}{ll}\text { Client: } & \text { C.P. } \\
\text { Prepared By: } & \text { A.W } \\
\text { Estimate Type: } & \text { Clas }\end{array}$ & $\begin{array}{l}\text { Ischay } \\
\text { Miller/s.n } \\
-5\end{array}$ & \\
\hline $\begin{array}{l}\text { timate } \\
\text { ubtotal } \\
\$ 83,027\end{array}$ & $\begin{array}{r}\text { Escalation } \\
\$ 7,697\end{array}$ & $\begin{array}{c}\text { Management } \\
\text { Reserve MR } \\
\$ 311,753\end{array}$ & $\begin{array}{l}\text { MR } \\
\% \\
35.00 \%\end{array}$ & $\frac{\text { TOTAL }}{\$ 122,477}$ \\
\hline$\$ 33,074$ & $\$ 3,066$ & $\$ 12,649$ & $35.00 \%$ & $\$ 48,789$ \\
\hline$\$ 33,074$ & $\$ 3,066$ & $\$ 12,649$ & $35.00 \%$ & $\$ 48,789$ \\
\hline$\$ 4,278$ & $\$ 397$ & $\$ 1,636$ & $35.00 \%$ & $\$ 6,310$ \\
\hline$\$ 4,278$ & $\$ 397$ & $\$ 1,636$ & $35.00 \%$ & $\$ 6,310$ \\
\hline$\$ 8,324$ & $\$ 772$ & $\$ 3,183$ & $35.00 \%$ & $\$ 12,279$ \\
\hline$\$ 76,796$ & $\$ 7,119$ & $\$ 29,370$ & $35.00 \%$ & $\$ 113,285$ \\
\hline$\$ 29,788$ & $\$ 2,761$ & $\$ 11,392$ & $35.00 \%$ & $\$ 43,941$ \\
\hline$\$ 29,788$ & $\$ 2,761$ & $\$ 11,392$ & $35.00 \%$ & $\$ 43,941$ \\
\hline$\$ 4,448$ & $\$ 412$ & $\$ 1,701$ & $35.00 \%$ & $\$ 6,562$ \\
\hline$\$ 4,448$ & $\$ 412$ & $\$ 1,701$ & $35.00 \%$ & $\$ 6,562$ \\
\hline$\$ 8,324$ & $\$ 772$ & $\$ 3,183$ & $35.00 \%$ & $\$ 12,279$ \\
\hline$\$ 68,624$ & $\$ 6,361$ & $\$ 26,245$ & $35.00 \%$ & $\$ 101,230$ \\
\hline$\$ 22,957$ & $\$ 2,128$ & $\$ 8,780$ & $35.00 \%$ & $\$ 33,865$ \\
\hline$\$ 22.957$ & $\$ 2,128$ & $\$ 8.780$ & $35.00 \%$ & $\$ 33.865$ \\
\hline$\$ 7,192$ & $\$ 667$ & $\$ 2,751$ & $35.00 \%$ & $\$ 10,610$ \\
\hline$\$ 7,192$ & $\$ 667$ & $\$ 2,751$ & $35.00 \%$ & $\$ 10,610$ \\
\hline$\$ 8,324$ & $\$ 772$ & $\$ 3,183$ & $35.00 \%$ & $\$ 12,279$ \\
\hline
\end{tabular}

\section{BEA}


Project Name: RTC Waste Heat Recovery

Project Summary Report

Project Location: ATR

\begin{tabular}{|c|c|c|}
\hline \multirow[t]{6}{*}{$\frac{\text { Level }}{5.1 .2 .2 .8}$} & $\underline{\text { Group }}$ & $\begin{array}{l}\text { Description } \\
\ldots \ldots \text { N.............. Neast Side Diesel Generator Room Metal } \\
\text { Priping and insulation Installation }\end{array}$ \\
\hline & & $\begin{array}{l}\ldots \ldots \ldots \ldots \ldots \ldots \\
\text { Area } 20^{\prime} \text { Elevation) }\end{array}$ \\
\hline & & 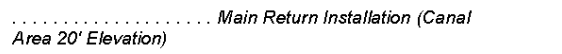 \\
\hline & & $\begin{array}{l}\text { Installation (Canal Area a } 20^{\circ} \text { Elevation) } \\
\text { insulation }\end{array}$ \\
\hline & & 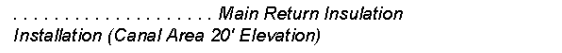 \\
\hline & & ................. Overhead Working Equipment \\
\hline 5.1 .2 .2 .9 & & ............. Piping, Valve, Coils, \& Control System \\
\hline 5.1.3 & & ........ Pump and Heat Exchanger Building \\
\hline \multirow[t]{4}{*}{ 5.1.3.1 } & & ........... Heat Exchanger and Pump House Excavation \\
\hline & & $\ldots \ldots \ldots \ldots$ Building Piping Vacuum Excavations \\
\hline & & ............... Pump House Building Foundation Excavation \\
\hline & & ............... Backill Excavations \\
\hline \multirow[t]{4}{*}{ 5.1.3.2 } & & ...... Heat Exchanger Concrete Footings/Foundation and \\
\hline & & .......... Building Concrete Footings \\
\hline & & $\ldots \ldots \ldots \ldots \ldots$ Building Concrete Foundation \\
\hline & & .......... Building Concrete Slab \\
\hline 5.1.3.3 & & .... Heat Exchanger Building Exterior \\
\hline 5.1.3.4 & & . Heat Exchanger Building Interior \\
\hline 5.1.3.5 & & Heat Exchanger Building HVAC \\
\hline \multirow[t]{4}{*}{ 5.1.3.6 } & & .......... Heat Exchanger Pump Building Electrical and Pump \\
\hline & & Connections \\
\hline & & ...... Electrical Building Service Connection \\
\hline & & $\ldots \ldots \ldots \ldots$ General Electrical Lighting and Outlets \\
\hline
\end{tabular}

$\begin{array}{ll}\text { Client: } & \text { C. P. Ischay } \\ \text { Prepared By: } & \text { A. W. Miller/S. N. Wasley }\end{array}$ Estimate Type: Class-5

\begin{tabular}{l} 
Estimate \\
Subtotal \\
\hline 556,303
\end{tabular}

$\frac{\text { Escalation }}{\$ 5,219}$

Management

$\frac{\text { Reserve MR }}{\$ 21,533} \frac{\%}{3500 \%}$

$\frac{\text { TOTAL }}{\$ 83,054}$

$\begin{array}{lllll}\$ 18,626 & \$ 1,727 & \$ 7,124 & 35.00 \% & \$ 27,477\end{array}$

$\begin{array}{lllll}\$ 18,626 & \$ 1,727 & \$ 7,124 & 35.00 \% & \$ 27,477\end{array}$

$\$ 5,363$

$\$ 497$

$\$ 2,051$

$35.00 \%$

$\$ 7,911$

$\$ 5,363$

$\$ 497$

$\$ 2,051$

$35.00 \%$

$\$ 7,911$

$\$ 8,324$

$$
\$ 772
$$

$\$ 3,183$

$35.00 \%$

$\$ 12,279$

$\$ 430,309$

$\$ 39,890$

$\$ 164,570$

$35.00 \%$

$\$ 634,769$

$\$ 123,780$

$35.00 \%$

$\$ 975,086$

BEA 
Project Summary Report

\begin{tabular}{|c|c|c|}
\hline Level & Group & $\frac{\text { Description }}{\text { Pump House Communication and Fire Alarm }}$ \\
\hline & & .. Primp nouse Lommunication and Fire Hiarm \\
\hline & & Pump \& Control Connections \\
\hline & & ............... Lightning Protection \\
\hline 5.1.3.7 & & $\ldots \ldots \ldots \ldots$ Building Fire Suppression System \\
\hline 5.1.4 & & ........ Waste Heat Recovery Tie-in \\
\hline 5.1.5 & & ........ Exchanger \& Pump Building Piping/Equipment \\
\hline 5.1.5.1 & & Heat Exchanger \\
\hline 5.1 .5 .2 & & ....... Heat Exchanger Piping \\
\hline 5.1.5.3 & & $\ldots \ldots \ldots \ldots$ Heat Exchanger Pumps \& Misc \\
\hline 5.2 & OPC & ....Provide Construction Support \\
\hline 5.2.1 & OPC & $\begin{array}{l}\text {.......Provide Safeguard and Security Support (Plan } \\
\text { Development) }\end{array}$ \\
\hline 5.2.2 & OPC & ........ Provide Operations Support for Outages \\
\hline 5.2 .3 & OPC & $\begin{array}{l}\text {....... Provide Radcon Support During Sub-k } \\
\text { Activities }\end{array}$ \\
\hline 5.2 .4 & OPC & ........ Provide for Security/Escorts/Guard Service \\
\hline 5.3 & OPC & ... Provide For Project Specific Training - Subcontractor \\
\hline 5.4 & OPC & .... Provide for 10 CFR 851 Requirements \\
\hline 5.5 & OPC & .... Misc Support During Execution \\
\hline 5.5.1 & OPC & $\begin{array}{l}\text {....... Provide AE Field Support Oversight during } \\
\text { Execution - Level of Effort (LOE) }\end{array}$ \\
\hline 0.5 .2 & OPC & $\begin{array}{l}\text {. . Le.... Provide AE Support oversignt auring Execution } \\
\text { Level offort (LOE) }\end{array}$ \\
\hline 6.0 & OPC & Environmental Assessment \\
\hline 7.0 & OPC & Operations \\
\hline 7.1 & OPC & .... Preventative Maintenance (PM) Plan Development \\
\hline 7.2 & OPC & .... Develop Operating Procedures, Manuals \& Documents \\
\hline
\end{tabular}

BEA

05/11/2012 11:08:29
Project Name: RTC Waste Heat Recovery

Project Location: ATR

\begin{tabular}{r} 
\\
Estimate \\
Subtotal \\
\hline$\$ 20,790$ \\
$\$ 138,580$ \\
$\$ 14,903$ \\
$\$ 58,762$ \\
$\$ 149,030$ \\
$\$ 674,373$ \\
$\$ 190,765$ \\
$\$ 287,719$ \\
$\$ 195,889$ \\
$\$ 350,862$ \\
$\$ 2,035$ \\
\\
$\$ 9,551$ \\
$\$ 202,266$ \\
$\$ \$ 137,011$ \\
$\$ 12,799$ \\
$\$ 17,940$ \\
$\$ 154,609$ \\
$\$ 133,289$ \\
\\
$\$ 21,320$ \\
$\$ 120,000$ \\
$\$ 184,408$ \\
$\$ 68,066$ \\
$\$ 87,452$ \\
\end{tabular}

Client:

C.P.Ischay

Prepared By: A. W. Miller/S. N. Wasley Estimate Type: Class-5

\begin{tabular}{|c|c|c|c|}
\hline$\frac{\text { scalation }}{\$ 1,927}$ & $\begin{array}{c}\text { Management } \\
\text { Reserve MR } \\
\$ 7,951\end{array}$ & $\begin{array}{l}\mathrm{MR} \\
\frac{\%}{35.00 \%}\end{array}$ & $\frac{\text { TOTAL }}{\$ 30,668}$ \\
\hline$\$ 12,846$ & $\$ 52,999$ & $35.00 \%$ & $\$ 204,426$ \\
\hline$\$ 1,382$ & $\$ 5,700$ & $35.00 \%$ & $\$ 21,984$ \\
\hline$\$ 5,447$ & $\$ 22,473$ & $35.00 \%$ & $\$ 86,683$ \\
\hline$\$ 13,815$ & $\$ 56,996$ & $35.00 \%$ & $\$ 219,840$ \\
\hline$\$ 62,514$ & $\$ 257,911$ & $35.00 \%$ & $\$ 994,798$ \\
\hline$\$ 17,684$ & $\$ 72,957$ & $35.00 \%$ & $\$ 281,406$ \\
\hline$\$ 26,672$ & $\$ 110,037$ & $35.00 \%$ & $\$ 424,427$ \\
\hline$\$ 18,159$ & $\$ 74,917$ & $35.00 \%$ & $\$ 288,965$ \\
\hline$\$ 32,525$ & $\$ 134,186$ & $35.00 \%$ & $\$ 517,573$ \\
\hline$\$ 189$ & $\$ 778$ & $35.00 \%$ & $\$ 3,002$ \\
\hline$\$ 885$ & $\$ 3,653$ & $35.00 \%$ & $\$ 14,089$ \\
\hline$\$ 18,750$ & $\$ 77,355$ & $35.00 \%$ & $\$ 298,371$ \\
\hline$\$ 12,701$ & $\$ 52,399$ & $35.00 \%$ & $\$ 202,111$ \\
\hline$\$ 1,186$ & $\$ 4,895$ & $35.00 \%$ & $\$ 18,880$ \\
\hline$\$ 1,663$ & $\$ 6,861$ & $35.00 \%$ & $\$ 26,465$ \\
\hline$\$ 14,332$ & $\$ 59,130$ & $35.00 \%$ & $\$ 228,071$ \\
\hline$\$ 12,356$ & $\$ 50,976$ & $35.00 \%$ & $\$ 196,621$ \\
\hline$\$ 1,9 / 6$ & 58,154 & $35.00 \%$ & $\$ 31,430$ \\
\hline$\$ 6,672$ & $\$ 44,335$ & $35.00 \%$ & $\$ 171,007$ \\
\hline$\$ 24,065$ & $\$ 72,966$ & $35.00 \%$ & $\$ 281,439$ \\
\hline$\$ 8,883$ & $\$ 26,932$ & $35.00 \%$ & $\$ 103,881$ \\
\hline$\$ 11,413$ & $\$ 34,603$ & $35.00 \%$ & $\$ 133,468$ \\
\hline
\end{tabular}




\begin{tabular}{|c|c|c|}
\hline$\frac{\text { Level }}{7.3}$ & $\frac{\text { Group }}{\text { OPC }}$ & ....Prepare $\frac{\text { Description }}{\text { Commissioning/so Test Plan }}$ \\
\hline 8.0 & OPC & SAR Update \\
\hline 9.0 & OPC & New System so Testing \\
\hline 10.0 & OPC & Readiness \\
\hline 10.1 & OPC & ....MSA \\
\hline 11.0 & OPC & PROJECT CLOSEOUT \\
\hline 11.1 & OPC & $\begin{array}{l}\text {.... Project Management (PM) Support during } \\
\text { Transition/Closeout Phase }\end{array}$ \\
\hline 11.1.1 & OPC & $\begin{array}{l}\text {....... Provide PM Oversight - Level of Effort (LOE) } \\
\text { during Transition/Closeout Phase }\end{array}$ \\
\hline 11.1.2 & OPC & ........ Closeout Project Files \\
\hline 11.2 & OPC & .... Closure Engineering and Design Documents \\
\hline 11.2.1 & OPC & $\begin{array}{l}\text {........ Prepare Master Facility As-Built Drawings and } \\
\text { incorporate into EDMS }\end{array}$ \\
\hline 11.2.2 & OPC & $\begin{array}{l}\text {........ Prepare Project As-Built Drawings and } \\
\text { incorporate into EDMS }\end{array}$ \\
\hline 11.3 & OPC & .... Closure Plans, Reports, and Documents \\
\hline 11.3.1 & OPC & Other Documents \\
\hline 11.3.1.1 & OPC & .......... Complete Closeout PM Checklist \\
\hline 11.3.1.2 & OPC & ........... Develop Lessons Learned \\
\hline 11.3.1.3 & OPC & .......... Prepare Final Project Closeout Report \\
\hline 11.4 & OPC & ....Project Acceptance and Closeout \\
\hline 11.4.1 & OPC & ........ Provide Operational Training \\
\hline 11.4.2 & OPC & $\begin{array}{l}\text {.......PProvide Start-Up Coordination, Materials, \& } \\
\text { supplies }\end{array}$ \\
\hline 11.4 .3 & OPC & ........ Provide Spares \\
\hline 12.0 & OPC & Heat Recovery System O\&M (5 Year) \\
\hline
\end{tabular}

Project Name: RTC Waste Heat Recovery

Project Location: ATR

Project Summary Report

\section{BEA}

Client: C. P. Ischay

Prepared By: A.W. Miller/S. N. Wasley Estimate Type: Class-5

Estimate

Subtotal

\begin{tabular}{c} 
Management \\
Reserve MR \\
\hline$\$ 11,431$
\end{tabular}

$\mathrm{MR}$

Escalation

$\frac{\%}{35.00 \%}$

$\frac{\text { TOTAL }}{\$ 44,091}$

$\$ 443,356$

$\$ 57,858$

$\$ 175,425$

$35.00 \%$

$\$ 676,639$

$\$ 34,452$

$\$ 4,496$

$\$ 13,632$

$35.00 \%$

$\$ 52,580$

$\$ 106,879$

$\$ 13,948$

$\$ 42,289$

$35.00 \%$

$\$ 163,116$

25,621

$\$ 42,289$

$35.00 \%$

$\$ 163,116$

$\$ 196,332$

$\$ 2,124$

$\$ 77,684$

$35.00 \%$

$\$ 299,638$

$\$ 14,554$

$\$ 1,899$

$\$ 6,440$

$35.00 \%$

$\$ 24,839$

$\$ 1,721$

$\$ 225$

$\$ 5,759$

$35.00 \%$

$\$ 22,212$

$\$ 34,010$

$\$ 4,438$

$\$ 681$

$35.00 \%$

$\$ \mathbf{\$ 1 3 , 4 5 7} \quad 35.00 \%$

$\$ 2,627$

$\$ 20,406$

$\$ 2,663$

$\$ 8,074$

$35.00 \%$

$\$ 13,604$

$\$ 1,775$

$\$ 5,383$

$35.00 \%$

$\$ 62,693$

$\$ 8,181$

$\$ 24,806$

$35.00 \%$

$\$ 20,762$

$\$ 45,725$

$\$ 8,181$
$\$ 5,967$

$\$ 24,806$

$35.00 \%$

$\$ 95,681$

$\$ 18,092$

$35.00 \%$

$\$ 95,681$

$\$ 5,716$

$\$ 746$

$\$ 2,262$

$35.00 \%$
$35.00 \%$

$\$ 83,354$

$\$ 30,323$

$\$ 10,878$
$\$ 3,957$

$\$ 32,981$

$35.00 \%$

$\$ 8,723$

$\$ 1,000$

$\$ 131$

ง990

$35.00 \%$

$\$ 127,213$

$\$ 52,031$

$\$ 449,869$

$\$ 6,790$

$\$ 20,588$

$\$ 178,002$

$35.00 \%$

$35.00 \%$

$\$ 686,579$ 
Project Name: RTC Waste Heat Recovery

Project Location: ATR

$\begin{array}{lll}\frac{\text { Level }}{12.1} & \frac{\text { Group }}{\text { OPC }} & \text {... System Mescription } \\ 12.2 & \text { OPC } & \text {... System Operating Costs (5 Years) }\end{array}$

12.2 OPC $\quad .$. System Operating Costs (5 Years)

Total RTC Waste Heat Recovery - INDIRECT
Project Summary Report

Client: C.P.Ischay

Client:
Prepared By: A. W. W Miller/S. N. Wasley
Estimate Type: Class-5

Estimate

$\begin{array}{lllll}\text { Subtotal } & \text { Escalation } & \text { Reserve MR } & \frac{\%}{\$ 367,705} & \frac{\text { TOTAL }}{\$ 145,492}\end{array}$

$\$ 82,164$

$\$ 10,722$

$35.00 \%$

$\$ 561,182$

$\$ 125,397$ 
Project Name: RTC Waste Heat Recovery

Project Location: ATR
Estimate Number: 7850
Estimate Markup Report

Prepared By: A. W. Miller/S. N. Wasley

Estimate Type: Class-5

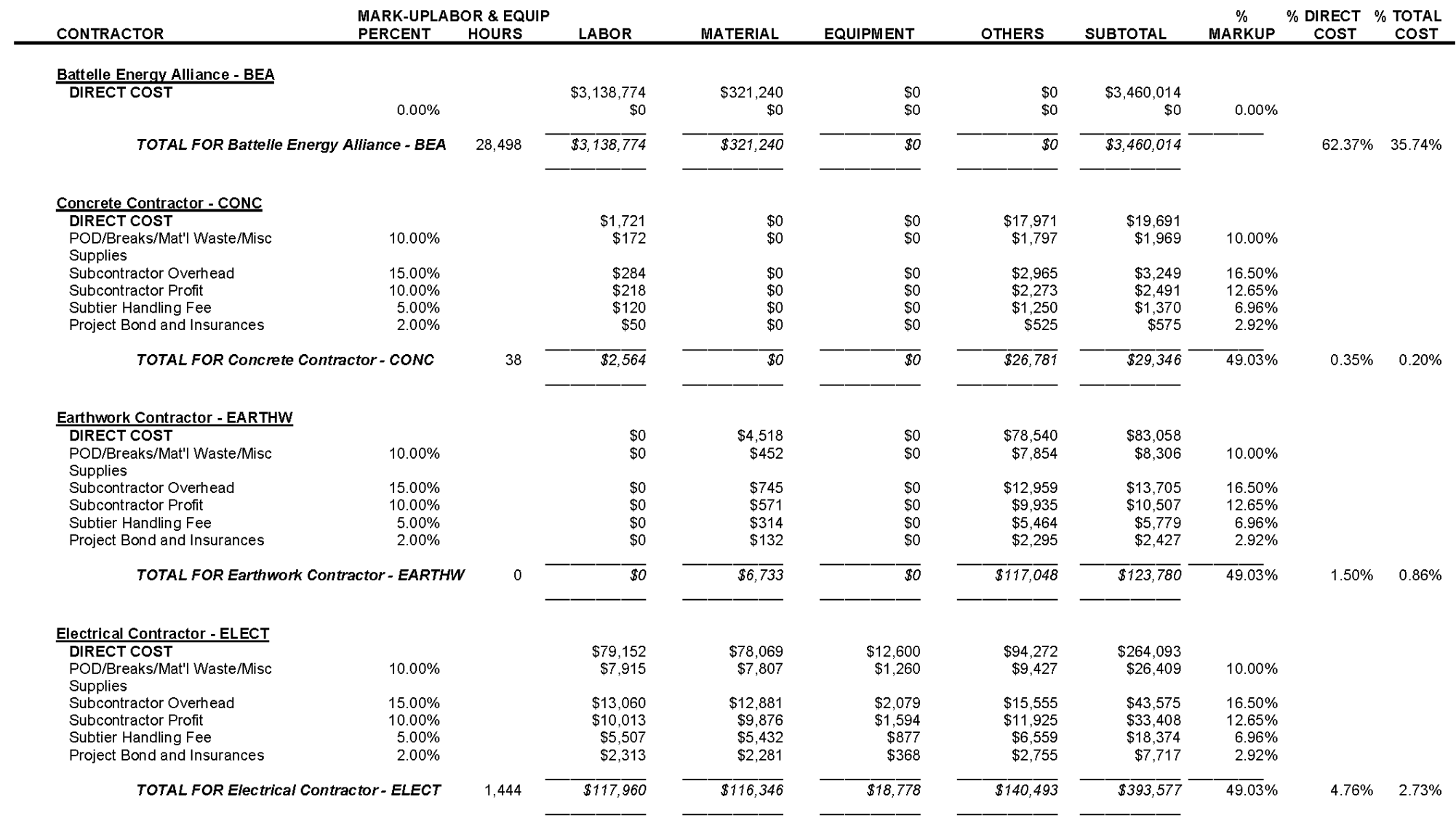

\section{BEA}




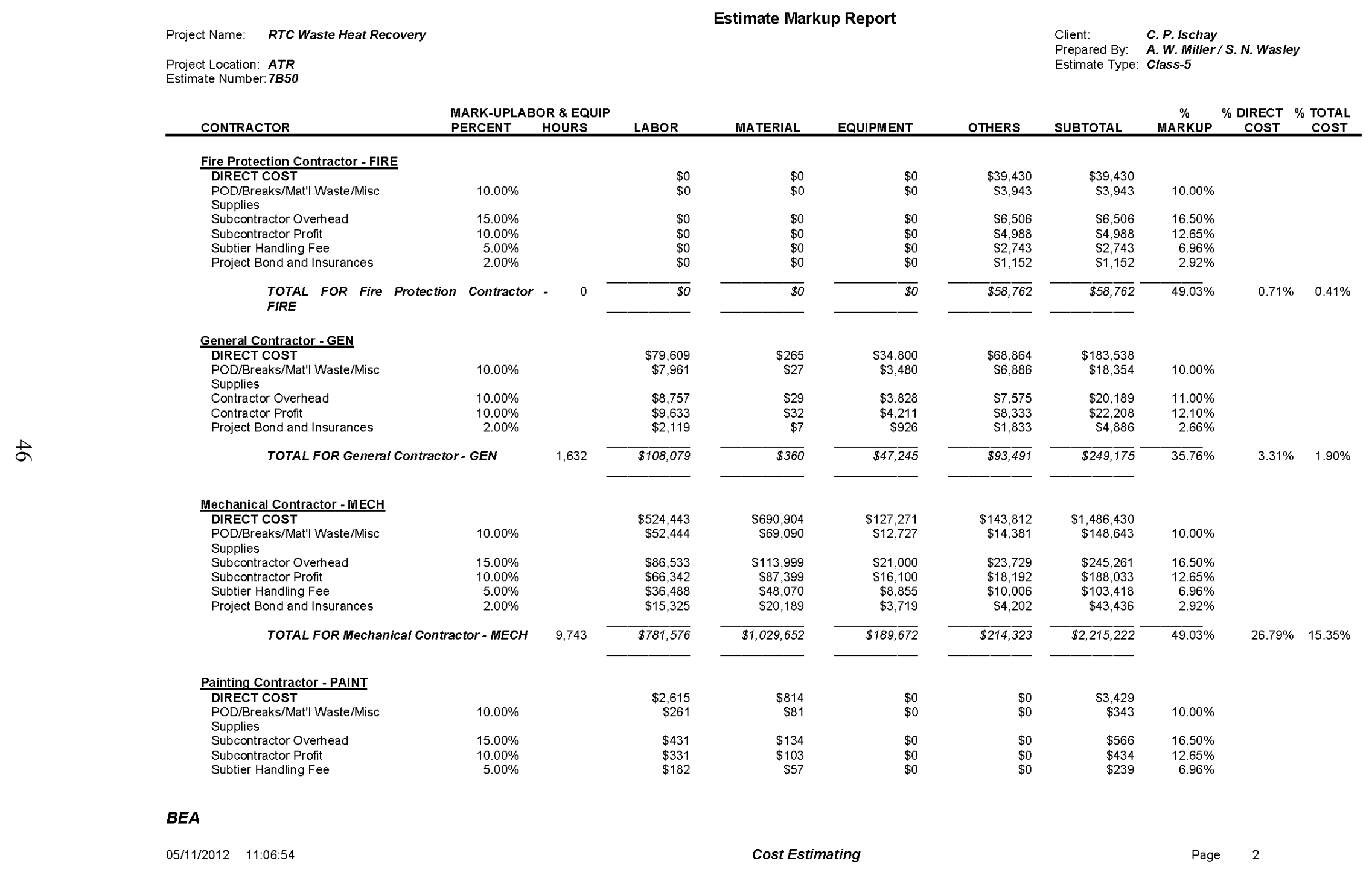




\section{Project Name: RTC Waste Heat Recovery}

Estimate Markup Report

Project Location: ATR

$\begin{array}{ll}\text { Client: } & \text { C.P. Ischay } \\ \text { Prepared By: } & \text { A. W. Miller/S. N. Wasley }\end{array}$

stimate Number:7850

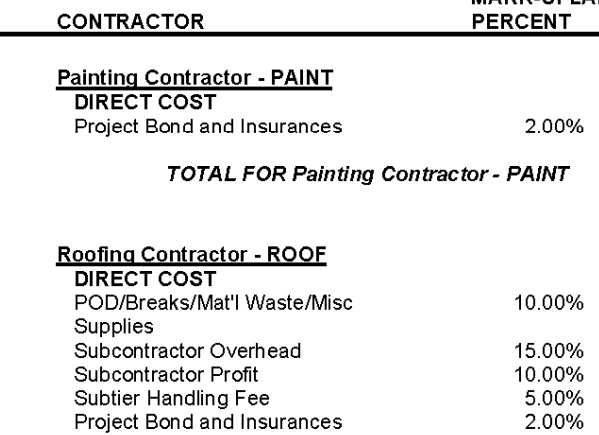

TOTAL FOR Roofing Contractor-ROOF

\section{Structural Steel Contractor - STEEL}

DIRECT COST
POD/Breaks/Mat'I Waste/Misc

Subcontractor Overhead

Subcontractor Profit

Subtier Handling Fee
Project Bond and Insurances

TOTAL FOR Structural Steel Contractor -

HOURS
HOUR

LABOR MATERIAL EQUIPMENT

Estimate Type: Class-5

60

$\$ 2,615$
$\$ 76$

$\begin{array}{r}\$ 814 \\ \$ 24 \\ -\$ 1.213 \\ \hline\end{array}$

$\$ 0$
$\$ 0$
$\$ 0$

OTHERS

\% DIRECT \% TOTAL

$\overline{\$ 3,897} \quad \overline{\$ 1,213}$

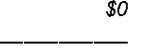

$\begin{array}{r}\$ 0 \\ \$ 0 \\ -\quad \$ 0 \\ \hline\end{array}$

$\begin{array}{r}\$ 3,429 \\ \$ 100 \\ \hline\end{array}$

$0.06 \% \quad 0.04 \%$

$00 \%$

$00 \%$
$00 \% \%$
$000 \%$

ROOF

0

$\$ 0$
$\$ 0$
$\$ 0$
$\$ 0$
$\$ 0$
$\$ 0$
$-\$ 0$
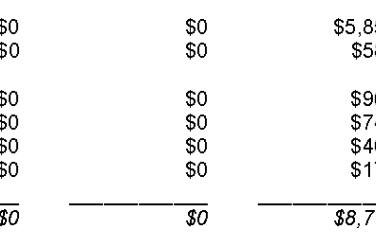

- $\$ 5$,

$49.03 \%$

so

-

- - -
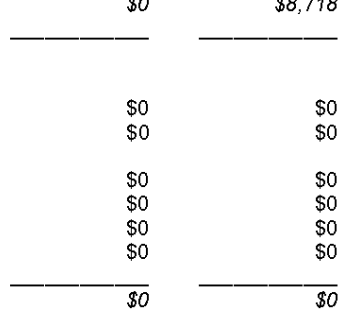

\begin{tabular}{rr}
$\$ 5,850$ & \\
$\$ 585$ & $10.00 \%$ \\
& \\
$\$ 965$ & $16.50 \%$ \\
$\$ 740$ & $12.65 \%$ \\
$\$ 407$ & $6.96 \%$ \\
$\$ 171$ & $2.92 \%$ \\
\hline$\$ 8,718$ & $49.03 \%$ \\
\hline & \\
\hline & \\
$\$ 2,426$ & \\
$\$ 243$ & $10.00 \%$ \\
$\$ 400$ & $16.50 \%$ \\
$\$ 307$ & $12.65 \%$ \\
$\$ 169$ & $6.96 \%$ \\
$\$ 71$ & $2.92 \%$ \\
\hline$\$ 3,615$ & $49.03 \%$ \\
\hline
\end{tabular}

$0.11 \% \quad 0.06 \%$

$2-\frac{\$ 1}{\$ 3,615}$

$\begin{array}{r}\$ 0 \\ -\$ 0 \\ \hline\end{array}$

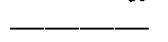

$0.04 \% \quad 0.03 \%$

\section{BEA}


Project Name: RTC Waste Heat Recovery

Project Location: $\mathbf{A T R}$

\section{Estimate Markup Report}

\begin{tabular}{|c|c|c|c|c|c|c|c|c|}
\hline \multirow[b]{2}{*}{$\begin{array}{l}\text { OR \& EQUIP } \\
\text { HOURS }\end{array}$} & \multirow[b]{2}{*}{ LABOR } & \multirow[b]{2}{*}{ MATERIAL } & \multirow[b]{2}{*}{ EQUIPMENT } & \multirow[b]{2}{*}{ OTHERS } & \multirow{2}{*}{$\begin{array}{l}\text { Client: } \\
\text { Prepared By: } \\
\text { Estimate Type: } \\
\text { SUBTOTAL }\end{array}$} & \multicolumn{3}{|c|}{$\begin{array}{l}\text { C. P. Ischay } \\
\text { A. W. Miller/S. N. Wasley } \\
\text { Class-5 }\end{array}$} \\
\hline & & & & & & $\begin{array}{c}\% \\
\text { MARKUP }\end{array}$ & $\begin{array}{c}\% \text { DIRECT } \\
\text { COST }\end{array}$ & $\begin{array}{c}\% \text { TOTAL } \\
\text { COST }\end{array}$ \\
\hline \multirow[t]{7}{*}{41,457} & $\$ 3,828,740$ & $\$ 1,095,810$ & $\$ 174,671$ & $\$ 448,739$ & $\$ 5,547,959$ & & $100.00 \%$ & \\
\hline & $\$ 327,725$ & $\$ 379,734$ & $\$ 81,023$ & $\$ 210,878$ & $\$ 999,361$ & & & $10.32 \%$ \\
\hline & $\$ 4,156,465$ & $\$ \$ 1,475,544$ & $\$ 255, \overline{694}$ & $\$ 659,617$ & $\$ 6,547,320$ & & & \\
\hline & $\$ 389,813$ & $\$ 148,794$ & $\$ 23,703$ & $\$ 61,146$ & $\$ 623,456$ & & & $6.44 \%$ \\
\hline & $\$ 4,546,278$ & $\$ 1,865,356$ & $\$ 316,841$ & $\$ 720,763$ & $\$ 7,170,776$ & & & \\
\hline & $\$ 1,591,197$ & $\$ 568,518$ & $\$ 97,789$ & $\$ 252,267$ & $\$ 2,509,772$ & & & $25.93 \%$ \\
\hline & $56,137,475$ & $\$ 2,433,874$ & $\$ 414,630$ & $\$ 973,030$ & $\$ 9,680,547$ & & & \\
\hline
\end{tabular}




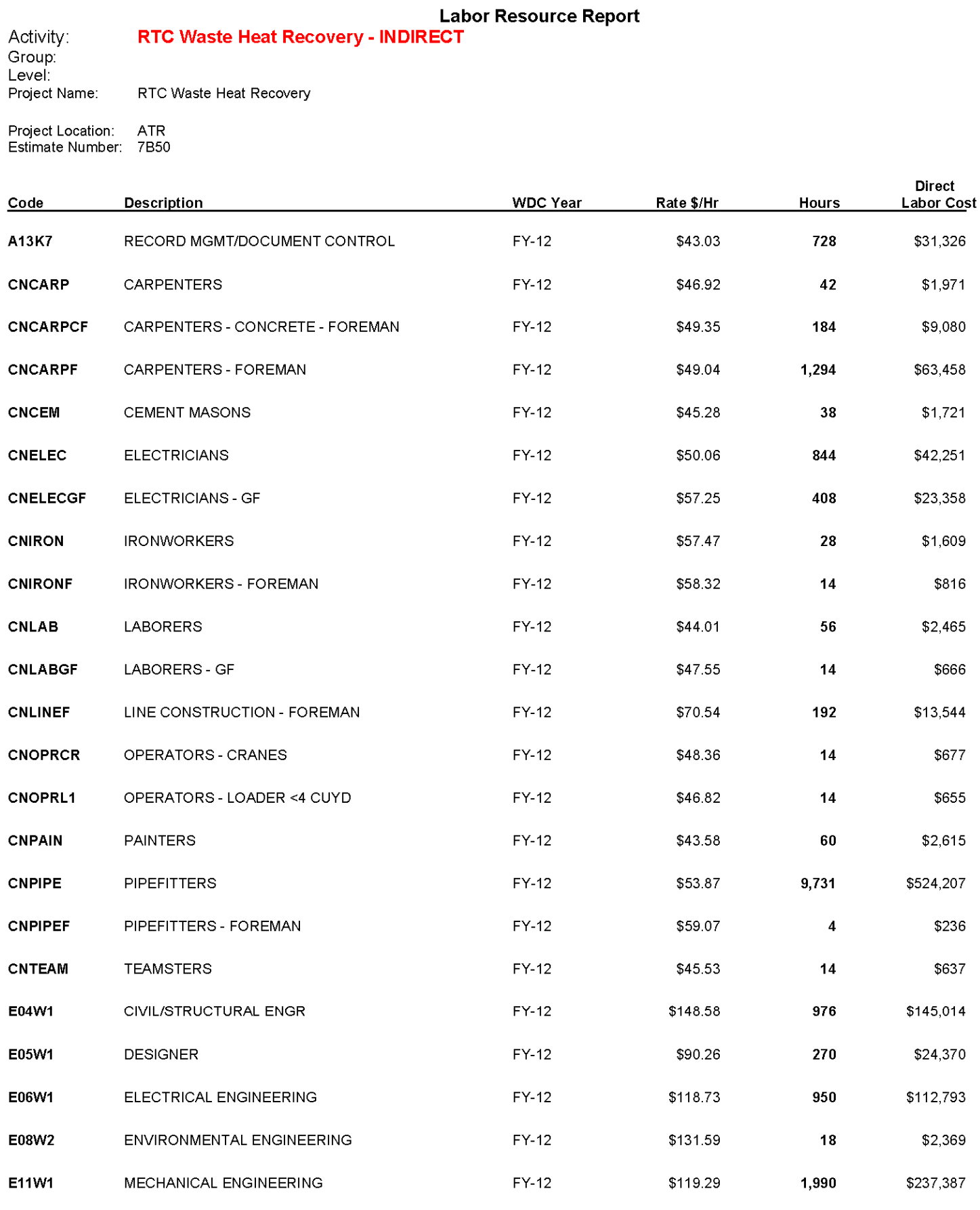

\section{BEA}




\section{Labor Resource Report}

Activity: $\quad$ RTC Waste Heat Recovery - INDIRECT

Group:
Level:

Project Name: RTC Waste Heat Recovery

Project Location: ATR

Estimate Number: 7B50

\begin{tabular}{|c|c|c|c|c|c|}
\hline Code & Description & WDC Year & Rate $\$ / \mathrm{Hr}$ & Hours & $\begin{array}{c}\text { Direct } \\
\text { Labor Cost }\end{array}$ \\
\hline E17W5 & QUALITY ENGINEERING & $\mathrm{FY}-12$ & $\$ 111.17$ & 140 & $\$ 15,564$ \\
\hline $\mathrm{E} 18 \mathrm{H} 6$ & RADIOLOGICAL ENGINEERING & FY-12 & $\$ 107.66$ & 60 & $\$ 6,460$ \\
\hline E19H1 & SAFETY ENGINEERING & FY-12 & $\$ 114.99$ & 1,270 & $\$ 146,037$ \\
\hline E27W4 & OTHER ENGINEERING & $\mathrm{FY}-12$ & $\$ 120.18$ & 30 & $\$ 3,605$ \\
\hline E32W1 & SYSTEM/SSC ENGINEER & FY-12 & $\$ 121.94$ & 800 & $\$ 97,552$ \\
\hline E34W2 & PROJECT ENGINEER & $\mathrm{FY}-12$ & $\$ 133.25$ & 640 & $\$ 85,280$ \\
\hline E35W1 & INSTRUMENTATION AND CONTROLS & FY-12 & $\$ 131.69$ & 300 & $\$ 39,507$ \\
\hline E41W1 & FIRE PROTECTION & $\mathrm{FY}-12$ & $\$ 126.49$ & 270 & $\$ 34,152$ \\
\hline E48W1 & OPERATIONS ENGINEER, GENERAL & FY-12 & $\$ 101.57$ & 420 & $\$ 42,659$ \\
\hline E54W4 & OPS SYSTEM ENGR, VITAL SAFETY SYSTEMS & $\mathrm{FY}-12$ & $\$ 119.49$ & 40 & $\$ 4,780$ \\
\hline E63W3 & NUCLEAR SAFETY ANALYSIS & FY-12 & $\$ 107.42$ & 2,080 & $\$ 223,434$ \\
\hline F05GB & FAC OPERATIONS & FY-12 & $\$ 109.78$ & 360 & $\$ 39,521$ \\
\hline F10GB & WORK PLANNING AND/OR SCHEDULING & $\mathrm{FY}-12$ & $\$ 79.42$ & 370 & $\$ 29,385$ \\
\hline F12M3 & SECURITY & FY-12 & $\$ 81.39$ & 25 & $\$ 2,035$ \\
\hline F22P2 & COST ESTIMATING & FY-12 & $\$ 88.12$ & 240 & $\$ 21,149$ \\
\hline F31J1 & CONSTRUCTION - OTHER TECHNICAL & $\mathrm{FY}-12$ & $\$ 91.32$ & 1,485 & $\$ 135,610$ \\
\hline F33P2 & PM SCHEDULING & FY-12 & $\$ 92.73$ & 583 & $\$ 54,015$ \\
\hline F35P1 & PROJECT MANAGER & FY-12 & $\$ 142.89$ & 2,070 & $\$ 295,782$ \\
\hline P37Y5 & TRAINING TECHNOLOGIST (CBT) & $\mathrm{FY}-12$ & $\$ 82.61$ & 85 & $\$ 7,022$ \\
\hline P44F2 & PLANNING AND CONTROLS & $\mathrm{FY}-12$ & $\$ 73.25$ & 566 & $\$ 41,441$ \\
\hline S06B3 & HEALTH PHYSICS & $\mathrm{FY}-12$ & $\$ 158.95$ & 320 & $\$ 50,864$ \\
\hline S21GC & REGULATORY COMPLIANCE - ENVIRONMENTAL & $\mathrm{FY}-12$ & $\$ 122.44$ & 40 & $\$ 4,898$ \\
\hline T02J1 & CONST INSPECT TECH & FY-12 & $\$ 79.14$ & 785 & $\$ 62,125$ \\
\hline
\end{tabular}

BEA 


\section{Labor Resource Report}

\begin{tabular}{|c|c|c|c|c|c|}
\hline \multirow{3}{*}{$\begin{array}{l}\text { Activity: } \\
\text { Group: } \\
\text { Level: } \\
\text { Project Name: } \\
\\
\text { Project Location: } \\
\text { Estimate Number: }\end{array}$} & \multicolumn{2}{|c|}{ RTC Waste Heat Recovery - INDIRECT } & \multirow[b]{4}{*}{ Rate $\$ / H r$} & \multirow[b]{4}{*}{ Hours } & \multirow[b]{4}{*}{$\begin{array}{c}\text { Direct } \\
\text { Labor Cos }\end{array}$} \\
\hline & RTC Waste Heat Recovery & & & & \\
\hline & $\begin{array}{l}\text { ATR } \\
7 \mathrm{~B} 50\end{array}$ & & & & \\
\hline Code & Description & WDC Year & & & \\
\hline T03W1 & DRAFTER & $\mathrm{FY}-12$ & $\$ 68.02$ & 1,460 & $\$ 99,309$ \\
\hline T04W2 & ELECTROMECH TECHNICIAN & $\mathrm{FY}-12$ & $\$ 76.38$ & 70 & $\$ 5,347$ \\
\hline T12W5 & QUALITY INSPECT TECH & $\mathrm{FY}-12$ & $\$ 68.04$ & 40 & $\$ 2,722$ \\
\hline T44GC & NUCLEAR FACILITY OPERATOR & FY-12 & $\$ 84.60$ & 40 & $\$ 3,384$ \\
\hline U11GB & ELECTRICIAN & FY-12 & $\$ 67.97$ & 140 & $\$ 9,516$ \\
\hline U16J1 & FITTER & $\mathrm{FY}-12$ & $\$ 67.21$ & 170 & $\$ 11,426$ \\
\hline U29GB & SYS MECHANIC & $\mathrm{FY}-12$ & $\$ 68.47$ & 2,740 & $\$ 187,608$ \\
\hline U52J1 & OPERATOR,UTILITY & $\mathrm{FY}-12$ & $\$ 75.72$ & 120 & $\$ 9,086$ \\
\hline $\mathrm{U} 60 \mathrm{H} 6$ & RADIOLOGICAL CONTROL TECH & FY-12 & $\$ 79.01$ & 2,560 & $\$ 202,266$ \\
\hline U96M4 & SECURITY POLICE OFFICER ॥ (SPO II) & $\mathrm{FY}-12$ & $\$ 53.52$ & 2,560 & $\$ 137,011$ \\
\hline $\mathrm{X} 18 \mathrm{H} 2$ & OCCUPATIONAL PHYSICIAN & FY-12 & $\$ 210.72$ & 38 & $\$ 7,902$ \\
\hline Z02GB & MANAGER, FAC SUPPORT SERVICES & $\mathrm{FY}-12$ & $\$ 124.05$ & 40 & $\$ 4,962$ \\
\hline Z03GB & MANAGER, OPERATIONS & $\mathrm{FY}-12$ & $\$ 141.05$ & 360 & $\$ 50,778$ \\
\hline Z04GD & MANAGER, SCI/ENG FUNCTION & $\mathrm{FY}-12$ & $\$ 161.74$ & 40 & $\$ 6,470$ \\
\hline Z04Q5 & MANAGER, SCI/ENG FUNCTION & FY-12 & $\$ 160.27$ & 40 & $\$ 6,411$ \\
\hline Zo4W4 & MANAGER, SCI/ENG FUNCTION & $\mathrm{FY}-12$ & $\$ 173.21$ & 160 & $\$ 27,714$ \\
\hline Z07M2 & SUPERVISOR, FAC SUPPORT SERVICES & $\mathrm{FY}-12$ & $\$ 72.81$ & 10 & $\$ 728$ \\
\hline
\end{tabular}

\section{BEA}


Project Name: RTC Waste Heat Recovery

Project Location: ATR
Estimate Number: 7850

DETAIL ITEM REPORT

1.2.1 Provide PM Oversignt - Level or Etrort (LOE) dunna Desian Pnase

Memo: Based on estimator judgment including consideration for the duration of the heat recovery system design phase.

\begin{tabular}{|c|c|c|c|c|c|c|c|c|c|}
\hline $\begin{array}{l}\text { Provide PM Oversight } \quad \text { BEA } \\
\text { \& Cost \& Schedule Monitoring during the Design }\end{array}$ & U. per Weeks & 24.00 Weeks & $\begin{array}{rl}20 & \$ 142.89 \\
480 & \mathrm{~F} 35 \mathrm{P} 1\end{array}$ & $\begin{array}{r}2867.8 \\
\$ 68,587\end{array}$ & $\begin{array}{l}0 \\
\$ 0\end{array}$ & $\begin{array}{l}0 \\
\text { \$o }\end{array}$ & $\begin{array}{r}0 \\
\$ 0\end{array}$ & \$o & $\begin{array}{r}2857.8 \\
\$ 68,587\end{array}$ \\
\hline $\begin{array}{l}\text { BEA } \\
\text { Procure Misc. Project Supplies, paper, signage, } \\
\text { office supplies, etc. for Design }\end{array}$ & Uc. periot & $1.00 \stackrel{\text { NM60 }}{\text { lot }}$ & 0 & $\$ 0$ & $\$ 0$ & $\begin{array}{r}500 \\
\$ 500\end{array}$ & $\begin{array}{l}0 \\
\$ 0\end{array}$ & \$o & $\$ 500$ \\
\hline $\begin{array}{l}\text { Subtotal } \\
\text { Sales Tax } \\
\text { Markups }\end{array}$ & $0.00 \%$ & & & $\begin{array}{r}\$ 68,587 \\
\$ 0 \\
\$ 0\end{array}$ & $\begin{array}{l}\$ 0 \\
\$ 0 \\
\$ 0\end{array}$ & $\begin{array}{r}\$ 500 \\
\$ 0 \\
\$ 0\end{array}$ & $\begin{array}{l}\$ 0 \\
\$ 0 \\
\$ 0\end{array}$ & $\begin{array}{l}\$ 0 \\
\$ 0 \\
\$ 0\end{array}$ & \\
\hline $\begin{array}{l}\text { Subtotal Estimate } \\
\text { sscalation } \\
\text { Management Reserve }\end{array}$ & & & & $\begin{array}{r}\$ 3,813 \\
\$ 25,340\end{array}$ & $\begin{array}{l}\$ 0 \\
\$ 0\end{array}$ & $\begin{array}{r}\$>8 \\
\$ 185\end{array}$ & $\$ 0$ & $\begin{array}{l}\$ 0 \\
\$ 0\end{array}$ & $\begin{array}{l}\$ 69,087 \\
\$ \$, 8,41 \\
\$ 25,525\end{array}$ \\
\hline otal 1.2.1 Provide PM Oversight - Level of Effort & & & & $\$ 97,741$ & \$o & $\$ 713$ & $\$ 0$ & $\$ 0$ & $\$ 98,4$ \\
\hline
\end{tabular}
during Design Phase

\subsubsection{Maintain Project Files - LOE during Design Phase}

Memo: Based on estimator judgment including consideration for the duration of the heat recovery system design phase.

\begin{tabular}{|c|c|c|c|c|c|c|c|c|c|}
\hline $\begin{array}{l}\text { BEA } \\
\text { Prepare Project Documents and Filing during Design } \\
\text { Phase }\end{array}$ & UC. perweoks & 24.00 weeks & $\begin{array}{rl}10 & \$ 43.03 \\
240 & \mathrm{~A} 13 \mathrm{~K} 7\end{array}$ & $\begin{array}{r}430.3 \\
\$ 10,327\end{array}$ & $\$ 0$ & $\$ 0$ & $\begin{aligned} 0 \\
\text { so }\end{aligned}$ & $\begin{array}{l}0 \\
\$ 0\end{array}$ & $\begin{array}{r}430,3 \\
\$ 10,327\end{array}$ \\
\hline $\begin{array}{l}\text { Subtotal } \\
\text { Sales Tax } \\
\text { Markups }\end{array}$ & $0.00 \%$ & & & $\begin{array}{r}\$ 10,327 \\
\$ 0 \\
\$ 0\end{array}$ & $\begin{array}{l}\$ 0 \\
\$ 0 \\
\$ 0\end{array}$ & $\begin{array}{l}\$ 0 \\
\$ 0 \\
\$ 0\end{array}$ & $\begin{array}{l}\$ 0 \\
\$ 0 \\
\$ 0\end{array}$ & $\begin{array}{l}\$ 0 \\
\$ 0 \\
\$ 0\end{array}$ & $\begin{array}{r}\$ 10,327 \\
\$ 0 \\
\$ 0\end{array}$ \\
\hline $\begin{array}{l}\text { Subtotal Estimate } \\
\text { Escalation } \\
\text { Management Reserve }\end{array}$ & & & & $\begin{array}{r}\$ 574 \\
\$ 3,815\end{array}$ & $\begin{array}{l}\$ 0 \\
\$ 0\end{array}$ & $\begin{array}{l}\$ 0 \\
\$ 0\end{array}$ & $\begin{array}{l}\$ 0 \\
\$ 0\end{array}$ & $\begin{array}{l}\$ 0 \\
\$ 0\end{array}$ & $\begin{array}{r}\$ 10,327 \\
\$ 574 \\
\$ 3,815\end{array}$ \\
\hline $\begin{array}{l}\text {-Total 1.2.2 Maintain Project Files - LOE during Design } \\
\text { Phase }\end{array}$ & & & & $\$ 14,717$ & so & so & \$o & so & $\$ 14,717$ \\
\hline
\end{tabular}

$\begin{array}{ll}\text { Client: } & \text { C. P. Ischay } \\ \text { Prepared By } & \text { A. W. Miller/s }\end{array}$

Estimate Type: Class-5

terial Subcontractor other TOTAL 
Project Name: RTC Waste Heat Recovery

Project Location: ATR

Estimate Number: 7850

\section{Code Description Contractor}

1.2.3 Provide Cost and Schedule Support dunna Desian Pnase

Based on estimator judgment including consideration for the duration of the heat recovery system design phase.

\begin{tabular}{|c|c|c|c|c|c|c|c|c|c|c|c|}
\hline $\begin{array}{r}\text { BEA } \\
\text { Prepare Level } 2 \text { Design Schedule }\end{array}$ & U.C. per activiti & 10.00 & activiti & $\begin{array}{r}0.25 \\
3\end{array}$ & $\begin{array}{l}573.25 \\
\text { P44F2 }\end{array}$ & $\begin{array}{r}18.313 \\
\$ 183\end{array}$ & $\$$ & $\begin{array}{r}0 \\
\$ 0\end{array}$ & $\begin{array}{r}0 \\
\$ 0\end{array}$ & $\begin{array}{l}0 \\
\$ 0\end{array}$ & $\begin{array}{l}18.313 \\
\$ 183\end{array}$ \\
\hline $\begin{array}{c}\text { BEA } \\
\text { Prepare Weekly Cost Status during Design }\end{array}$ & U. per weeks & 24.00 & weeks & $\begin{array}{r}10 \\
240\end{array}$ & $\begin{array}{l}573.25 \\
\text { P44F2 }\end{array}$ & $\begin{array}{r}732.5 \\
\$ 17,580\end{array}$ & $\$ 0$ & $\$ 0$ & $\begin{array}{l}0 \\
\$ 0\end{array}$ & $\$ 0$ & $\begin{array}{r}732.5 \\
\$ 17,580\end{array}$ \\
\hline $\begin{array}{l}\text { BEA } \\
\text { Prepare Weekly Schedule Status during Design }\end{array}$ & uc. per weeks & 24.00 & weeks & $\begin{array}{r}10 \\
240\end{array}$ & $\begin{array}{l}\$ 92.73 \\
\mathrm{~F} 33 \mathrm{P} 2\end{array}$ & $\begin{array}{r}927.3 \\
\$ 22,255\end{array}$ & $\begin{array}{l}0 \\
\$ 0\end{array}$ & $\begin{array}{r}0 \\
\$ 0\end{array}$ & $\begin{array}{c}0 \\
\$ 0\end{array}$ & $\begin{array}{l}0 \\
\$ 0\end{array}$ & $\begin{array}{r}927.3 \\
\$ 22,255\end{array}$ \\
\hline $\begin{array}{c}\text { BEA } \\
\text { Set Up Activity Charge Numbers for Design }\end{array}$ & U.C pernumber & 5.00 & number & $\begin{array}{r}0.25 \\
1\end{array}$ & $\begin{array}{l}\$ 73.25 \\
\text { P44F2 }\end{array}$ & $\begin{array}{r}18.312 \\
\$ 92\end{array}$ & $\begin{array}{l}0 \\
\$ 0\end{array}$ & $\begin{array}{r}0 \\
\$ 0\end{array}$ & $\begin{array}{r}0 \\
\$ 0\end{array}$ & $\begin{array}{r}0 \\
\$ 0\end{array}$ & $\begin{array}{r}18.312 \\
\$ 92\end{array}$ \\
\hline $\begin{array}{l}\text { BEA } \\
\text { Develop Class-2 Cost Estimate to Support } \\
\text { Construction \& Procurement Awards }\end{array}$ & U.C. per Allow & 1.00 & Allow & $\begin{array}{r}120 \\
120\end{array}$ & $\begin{array}{l}\$ 88.12 \\
F 22 P 2\end{array}$ & $\begin{array}{r}\$ 0574,4 \\
\$ 10,574\end{array}$ & so & $\begin{array}{r}0 \\
\text { so }\end{array}$ & so & so & $\begin{array}{r}10574.4 \\
\$ 10,574\end{array}$ \\
\hline $\begin{array}{l}\text { total } \\
\text { st Tax } \\
\text { kups }\end{array}$ & $0.00 \%$ & & & & & $\begin{array}{r}\$ 50,684 \\
\$ 0 \\
\$ 0\end{array}$ & $\begin{array}{l}\$ 0 \\
\$ 0 \\
\$ 0\end{array}$ & $\begin{array}{l}\$ 0 \\
\$ 0 \\
\$ 0\end{array}$ & $\begin{array}{l}\$ 0 \\
\$ 0 \\
\$ 0\end{array}$ & $\begin{array}{l}\$ 0 \\
\$ 0 \\
\$ 0\end{array}$ & $\begin{array}{r}\$ 50,684 \\
\$ 0 \\
\$ 0\end{array}$ \\
\hline $\begin{array}{l}\text { total Estimate } \\
\text { alation } \\
\text { algement Reserve }\end{array}$ & & & & & & $\begin{array}{r}\$ 2,818 \\
\$ 18,726\end{array}$ & $\begin{array}{l}\$ 0 \\
\$ 0\end{array}$ & $\begin{array}{l}\$ 0 \\
\$ 0\end{array}$ & $\begin{array}{l}\$ 0 \\
\$ 0\end{array}$ & $\begin{array}{l}\$ 0 \\
\$ 0\end{array}$ & $\begin{array}{r}\$ 50,684 \\
\$ 2,818 \\
\$ 18,726\end{array}$ \\
\hline $\begin{array}{l}\text { 1.2.3 Provide Cost and Schedule Support } \\
\text { Phase }\end{array}$ & & & & & & $\$ 72,228$ & \$o & $\$ 0$ & $\$ 0$ & \$o & \\
\hline
\end{tabular}

DETAIL ITEM REPORT

lient: $\quad$ C. P. Ischay

Estimate Type: Class-5

Equipment Material Subcontractor other TOTAL
Prepared By: A. W. Miller/S. N. Wasley

\subsubsection{Provide PM Oversight - Level of Effort (LOE) during Execution}

Memo: Based on estimator judgment including consideration for the duration of the heat recovery system installation execution phase.

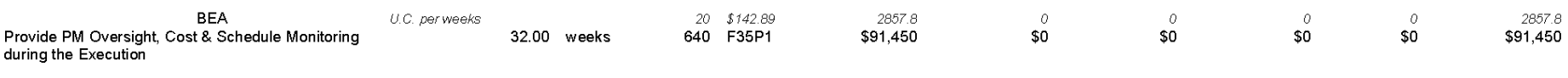


Project Name: RTC Waste Heat Recovery

Project Location: ATR

Estimate Number: 7850

Code Description

1.3.1 Provide PNI Oversight - Level or ETrort (LOE) aunng Execution

\begin{tabular}{|c|c|c|c|c|c|c|c|c|c|}
\hline 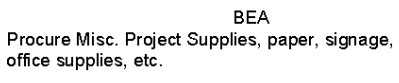 & Uc. periot & $\begin{array}{cc}\text { NM60 } \\
1.00\end{array}$ & 0 & $\$ 0$ & \$0 & $\begin{array}{r}250 \\
\$ 250\end{array}$ & $\begin{array}{l}0 \\
\$ 0\end{array}$ & $\begin{array}{l}0 \\
\$ 0\end{array}$ & $\begin{array}{r}250 \\
\$ 250\end{array}$ \\
\hline $\begin{array}{l}\text { total } \\
\text { s Tax } \\
\text { sups }\end{array}$ & $0.00 \%$ & & & $\begin{array}{r}\$ 91,450 \\
\$ 0 \\
\$ 0\end{array}$ & $\begin{array}{l}\$ 0 \\
\$ 0 \\
\$ 0\end{array}$ & $\begin{array}{r}\$ 250 \\
\$ 0 \\
\$ 0\end{array}$ & $\begin{array}{l}\$ 0 \\
\$ 0 \\
\$ 0\end{array}$ & $\begin{array}{l}\$ 0 \\
\$ 0 \\
\$ 0\end{array}$ & $\begin{array}{r}\$ 91,700 \\
\$ 0 \\
\$ 0\end{array}$ \\
\hline $\begin{array}{l}\text { total Estimate } \\
\text { alation } \\
\text { agement Reserve }\end{array}$ & & & & $\begin{array}{r}\$ 8,477 \\
\$ 34,974\end{array}$ & $\begin{array}{l}\$ 0 \\
\$ 0\end{array}$ & $\begin{array}{l}\$ 23 \\
\$ 96\end{array}$ & $\begin{array}{l}\$ 0 \\
\$ 0\end{array}$ & $\begin{array}{l}\$ 0 \\
\$ 0\end{array}$ & $\begin{array}{r}\$ 91,700 \\
\$ 8,501 \\
\$ 35,070\end{array}$ \\
\hline 1.3.1 Provide PM Ov & & & & $\$ 134,901$ & $\$ 0$ & $\$ 369$ & $\$ 0$ & $\$ 0$ & $\$ 135,270$ \\
\hline
\end{tabular}

1.3.2 Maintain Project Files - LOE during Execution

Memo: Based on estimator judgment including consideration for the duration of the heat recovery system installation execution phase.

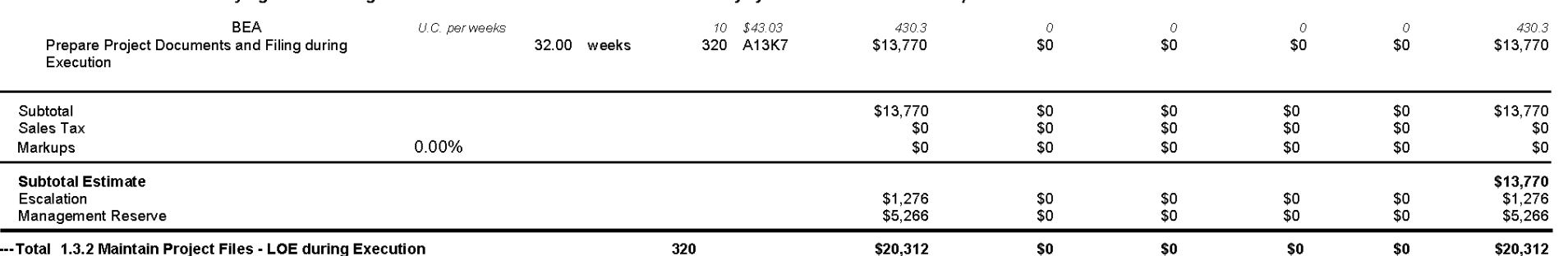


Project Name: RTC Waste Heat Recovery

Project Location: ATR

Primate Number: 7850

Code Description

1.3.3 Provide Costand Schequle Support aunng Execurtion

Mero:

\begin{tabular}{|c|c|c|c|c|c|c|c|c|c|c|c|}
\hline $\begin{array}{l}\text { BEA } \\
\text { Prepare Level } 4 \text { Execution and Monitoring Schedule }\end{array}$ & UC. peractiviti & 10.00 & activiti & & $\begin{array}{l}\$ 92.73 \\
F 33 P 2\end{array}$ & $\begin{array}{r}23.183 \\
\$ 232\end{array}$ & \$0 & $\begin{array}{l}0 \\
\$ 0\end{array}$ & $\begin{array}{r}0 \\
\$ 0\end{array}$ & $\$ 0$ & $\begin{aligned} 23.193 \\
\$ 232\end{aligned}$ \\
\hline $\begin{array}{c}\text { BEA } \\
\text { Prepare Weekly Cost Status during Execution }\end{array}$ & U.c. per weeks & 32.00 & weeks & $\begin{array}{r}10 \\
320\end{array}$ & $\begin{array}{l}\$ 73.25 \\
\text { P44F2 }\end{array}$ & $\begin{array}{r}732.5 \\
\$ 23,440\end{array}$ & $\$ 0$ & \$o & \$o & \$o & $\$ 23,440$ \\
\hline $\begin{array}{l}\text { BEA } \\
\text { Prepare Weekly Schedule Status during Design }\end{array}$ & uc perweeks & 32.00 & weeks & $\begin{array}{r}10 \\
320\end{array}$ & $\begin{array}{l}\$ 92.73 \\
\mathrm{~F} 33 \mathrm{P} 2\end{array}$ & $\begin{array}{r}927.3 \\
\$ 29,674\end{array}$ & $\$$ & $\begin{array}{l}0 \\
\$ 0\end{array}$ & $\begin{array}{r}0 \\
\$ 0\end{array}$ & $\$ 0$ & $\begin{array}{r}927.3 \\
\$ 29,674\end{array}$ \\
\hline $\begin{array}{c}\text { BEA } \\
\text { Set Up Activity Charge Numbers for Execution }\end{array}$ & UC. per number & 8.00 & number & $\begin{array}{r}0.25 \\
2\end{array}$ & $\begin{array}{l}\$ 73.25 \\
\text { P44F2 }\end{array}$ & $\begin{array}{r}\begin{array}{r}18.313 \\
\$ 147\end{array} \\
\$\end{array}$ & $\begin{array}{l}0 \\
\$ 0\end{array}$ & $\$$ & $\begin{aligned} 0 \\
\$ 0\end{aligned}$ & $\begin{array}{l}0 \\
\$ 0\end{array}$ & $\begin{array}{r}18.313 \\
\$ 147\end{array}$ \\
\hline $\begin{array}{l}\text { Subtotal Estimate } \\
\text { Escalation } \\
\text { Management Reserve }\end{array}$ & & & & & & $\begin{array}{r}\$ 4,959 \\
\$ 20,458\end{array}$ & 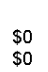 & $\begin{array}{l}\$ 0 \\
\$ 0\end{array}$ & $\begin{array}{l}\$ 0 \\
\$ 0\end{array}$ & $\begin{array}{l}\$ 0 \\
\$ 0\end{array}$ & $\begin{array}{l}\$ 53,492 \\
\$ 4,959 \\
\$ 20,458\end{array}$ \\
\hline $\begin{array}{l}\text { Total 1.3.3 Provide Cost and Schedule Support } \\
\text { Execution }\end{array}$ & & & & & & $\$ 78,908$ & $\$ 0$ & $\$ 0$ & \$o & $\$ 0$ & \\
\hline
\end{tabular}

DETAIL ITEM REPORT

Client: $\quad$ C. P. Ischay

Prepared By: A.W. Miller/S. N. Wasley

Estimate Type: Class-5

rial Subcontractor

Other TOTAL
Execution

\subsection{Provide Quallity Inspections During Construction - First Line}

Memo: Based on estimator judgment including consideration for the duration of the heat recovery system, installation execution phase for qualiny assurance duties.
$\mathrm{BE} \wedge$
Provide On-Site Inspection during Construction
u. porwooks 32.00 weeks
$\begin{array}{rr}20 & \$ 70.14 \\ 640 & \text { T02.J1 }\end{array}$
$\$ 50,650$
$\$ 0$
0
$\$ 0$
0
$\$ 0$
$\begin{array}{r}0 \\ \$ 0\end{array} \quad \$ 50,650$ 
Project Name: RTC Waste Heat Recovery

Project Location: ATR

Estimate Number: 7850

Code Descriptio

1.4.1 Provide Qualliv Inspections During Construction - First Lin

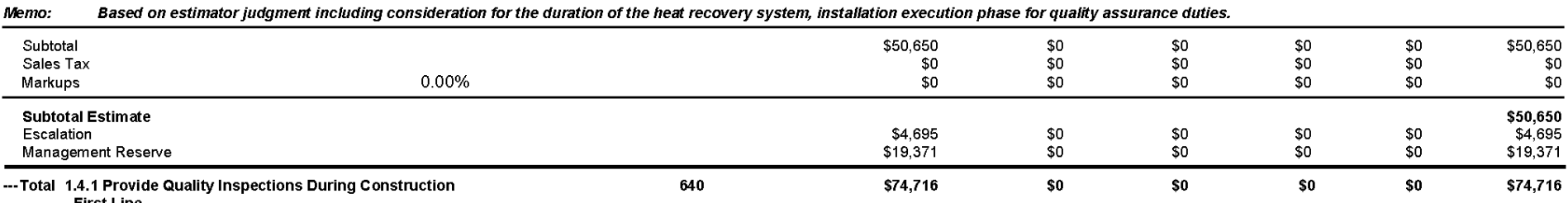

1.4.1 Provide Quality Inspections During Construction
- First Line

DETAIL ITEM REPORT

C.P.Ischay

Prepared By: A.W.Miller/S. N. Wasley

Estimate Type: Class-5 Qty UOM Hrs Resource Labor Equipment Material Subcontractor Other TOTAL $\$ 0$ 50,650
$\$ 4,695$
19,371

1.4.2 Quality Assurance Oversight

Memo: Based on estimator's judgment

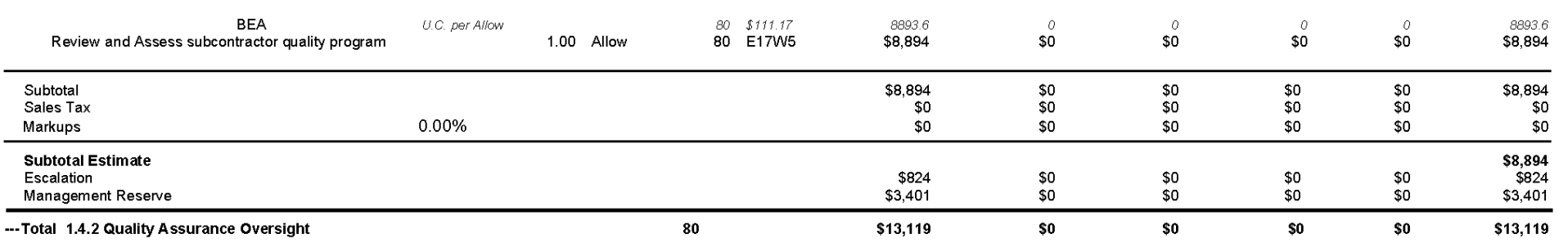

\subsection{Provide $\mathrm{CM}$ Oversight/Support during Execution - Level of Effort (LOE)}

Memo: Construction management coverage is based on conversation with $R$. Strong (BEA Construction Manager). Allowance is based on fulf time coverage to support all subcontract work

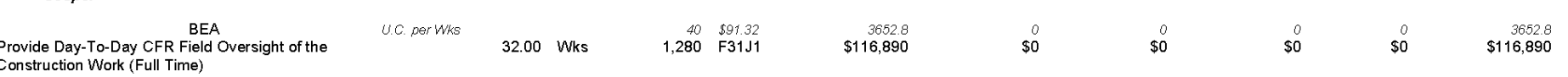


Project Name: RTC Waste Heat Recovery

Project Location: ATR

Pste 7850

Code Description

1.1 Provide CM Oversiantisupport dunna Execution - Level or ETron tLo日

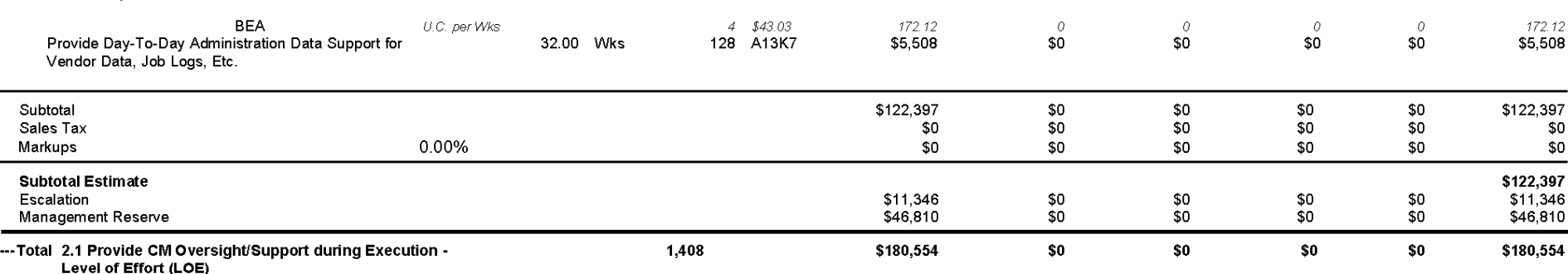

\subsection{BEA - Provide CM Supervision - OPC}

Memo: Construction management coverage is based on conversation with R. Strong (BEA Construction Manager).

\begin{tabular}{|c|c|c|c|c|c|c|c|c|c|}
\hline Provide Overall Project CM Supervision & uc. perwks & 32.00 wks & $\begin{array}{rr}5 & \$ 91.32 \\
160 & \mathrm{~F} 31 \mathrm{J1}\end{array}$ & $\begin{array}{r}456.6 \\
\$ 14,611\end{array}$ & $\begin{array}{l}0 \\
\$ 0\end{array}$ & $\$ 0$ & $\begin{array}{l}0 \\
\$ 0\end{array}$ & $\$ 0$ & $\begin{array}{r}456,6 \\
\$ 14,611\end{array}$ \\
\hline Total 2.2 BEA - Provide CM Supervi & & & & $\$ 21,554$ & \$0 & $\$ 0$ & $\$ 0$ & so & $\$ 21,554$ \\
\hline
\end{tabular}


Project Name: RTC Waste Heat Recovery

Project Location: ATR

Estimate Number: 7850

Code Description

2.3 Provlde Sarety oversiantsupport aunna Execution - Lo

Memo: Construction management coverage is based on conversation with R. Strong (BEA Construction Manager). Allowance is based on $3 / 4$ time coverage during the execution phase of the subcontracted work

\begin{tabular}{|c|c|c|c|c|c|c|c|c|c|}
\hline $\begin{array}{l}\text { BEA } \\
\text { Provide Part-Time Safety Oversight (3/4 Time) }\end{array}$ & uc. perwhs & 32.00 Wks & $\begin{array}{rr}30 & \$ 114.99 \\
960 & \mathrm{E} 19 \mathrm{H} 1\end{array}$ & $\begin{array}{r}3449.7 \\
\$ 110,390\end{array}$ & $\begin{array}{l}0 \\
\$ 0\end{array}$ & $\begin{array}{l}0 \\
\$ 0\end{array}$ & $\begin{array}{r}0 \\
\$ 0\end{array}$ & \$0 & $\begin{array}{r}3449.7 \\
\$ 110,390\end{array}$ \\
\hline Total 2.3 Provide Safety Oversight/Support & ution & & & $\$ 162,842$ & \$o & \$o & $\$ 0$ & so & $\$ 162,842$ \\
\hline
\end{tabular}

\subsection{Provide Industrial Hygiene Oversight/Support during Execution - LOE}

Memo: Construction management coverage is based on conversation with $R$. Strong (BEA construction Manager). Allowance is based on quarter time coverage during the execution of the

\begin{tabular}{|c|c|c|c|c|c|c|c|c|c|}
\hline $\begin{array}{l}\text { BEA } \\
\begin{array}{l}\text { Provide Air-Borne IH Dust Checks ( } 1 / 4 \text { Time } \\
\text { Coverage) }\end{array}\end{array}$ & UC. per Whs & 32.00 Wks & $\begin{aligned} 10 & \$ 158.95 \\
320 & \$ 06 B 3\end{aligned}$ & $\begin{array}{r}1589.5 \\
\$ 50,864\end{array}$ & $\$$ & $\$$ & $\$ 0$ & $\$ 0$ & $\begin{array}{r}1589.5 \\
\$ 50,864\end{array}$ \\
\hline $\begin{array}{l}\text { Subtotal } \\
\text { Sales Tax } \\
\text { Markups }\end{array}$ & $0.00 \%$ & & & $\begin{array}{r}\$ 50,864 \\
\$ 0 \\
\$ 0\end{array}$ & $\begin{array}{l}\$ 0 \\
\$ 0 \\
\$ 0\end{array}$ & $\begin{array}{l}\$ 0 \\
\$ 0 \\
\$ 0\end{array}$ & $\begin{array}{l}\$ 0 \\
\$ 0 \\
\$ 0\end{array}$ & $\begin{array}{l}\$ 0 \\
\$ 0 \\
\$ 0\end{array}$ & $\begin{array}{r}\$ 50,864 \\
\$ 0 \\
\$ 0\end{array}$ \\
\hline $\begin{array}{l}\text { Subtotal Estimate } \\
\text { Escalation } \\
\text { Management Reserve }\end{array}$ & & & & $\begin{array}{r}\$ 4,715 \\
\$ 19,453\end{array}$ & $\begin{array}{l}\$ 0 \\
\$ 0\end{array}$ & $\begin{array}{l}\$ 0 \\
\$ 0\end{array}$ & $\begin{array}{l}\$ 0 \\
\$ 0\end{array}$ & $\begin{array}{l}\$ 0 \\
\$ 0\end{array}$ & $\begin{array}{r}\$ 50,864 \\
\$ 4,715 \\
\$ 19,453\end{array}$ \\
\hline $\begin{array}{l}\text { Total 2.4 Provide Industrial Hygiene } 0 \\
\text { during Execution - LOE }\end{array}$ & & & & $\$ 75,032$ & \$o & so & $\$ 0$ & so & $\$ 75,032$ \\
\hline
\end{tabular}

Client:

Prepared By: A. W. Miller/S. N. Wasle

Subcontractor Other TOTAL

DETAIL ITEM REPORT
Estimate Type: Class-5 
Project Name: RTC Waste Heat Recovery

Project Location: ATR

Code Description

Qty UOM Hrs Resource Labor

\section{Equipment}

\section{$\underline{\text { Material }} \underline{\text { Subcontractor }}$}

C. P. Ischay $\quad$ ient:

Prepared By. A.W. Miller/S. N. Wasley

stimate Type: Class-5

2.5 Pertorm Subsurtace Investlgations Pnor to Excav'ns. Floor and wall Penetrations

Memo: The detail items, resources, and productivities are based upon discussions with $R$. Strong, construction management. Kevin Wells is lead technician for subsurface investigations.

BEA

$\begin{array}{lr}2.5 & 579.14 \\ 25 & \text { T02.J }\end{array}$

197.85
$\$ 1,979$

$\$ 0$

0
$\$ 0$

$\$ 0$

$\begin{array}{lrr}0 & 0 & 197.85 \\ \$ 0 & \$ 0 & \$ 1,979\end{array}$

Prepare subsurface investigEA

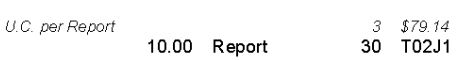

237.42
$\$ 2,374$

$\$ 0$

0
$\$ 0$

$\begin{array}{rrr}0 & 0 & 23742 \\ \$ 0 & \$ 0 & \$ 2,374\end{array}$

Memo: Downloading of information from the subsurface equipment and preparation of proper technical report requires approximately 3 hours per location.

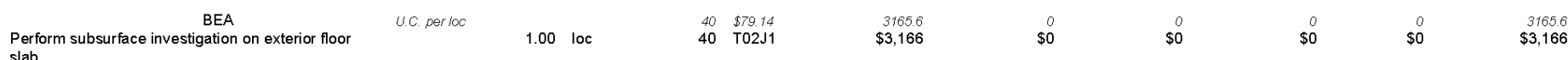

Memo: The duration of this activity, 2.5 hours per location, is based on the estimator's judgment.

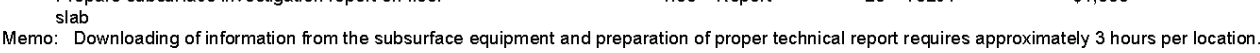

\begin{tabular}{|c|c|c|c|c|c|c|c|c|c|c|c|}
\hline $\begin{array}{l}\text { EEA } \\
\text { Perform subsurface investigation on interior floor } \\
\text { slab }\end{array}$ & U.C per Area & 1.00 & Area & $\begin{array}{l}20 \\
20\end{array}$ & $\begin{array}{l}\$ 79.14 \\
\text { T02J1 }\end{array}$ & $\begin{array}{r}1582.8 \\
\$ 1,583\end{array}$ & $\$ 0$ & $\$ 0$ & $\$ 0$ & \$o & $\begin{array}{r}1582.8 \\
\$ 1,583\end{array}$ \\
\hline $\begin{array}{l}\text { BEA } \\
\begin{array}{l}\text { Prepare subsurface investigation report on floor } \\
\text { slab }\end{array}\end{array}$ & U. per Report & 1.00 & Report & $\begin{array}{c}10 \\
10\end{array}$ & $\begin{array}{l}\$ 79.14 \\
\text { TO2J1 }\end{array}$ & $\begin{array}{l}791.4 \\
\$ 791\end{array}$ & $\begin{array}{l}0 \\
\$ 0\end{array}$ & $\begin{array}{l}0 \\
\$ 0\end{array}$ & $\begin{array}{l}0 \\
\$ 0\end{array}$ & \$0 & $\begin{array}{l}791.4 \\
\$ 791\end{array}$ \\
\hline
\end{tabular}

\begin{tabular}{|c|c|c|c|c|c|c|c|}
\hline $\begin{array}{l}\text { Subtotal } \\
\text { Salos Tax }\end{array}$ & & $\begin{array}{r}\$ 11,475 \\
\$ 0\end{array}$ & $\begin{array}{l}\$ 0 \\
\$ 0\end{array}$ & $\begin{array}{l}\$ 0 \\
\$ 0\end{array}$ & $\begin{array}{l}\$ 0 \\
\$ 0\end{array}$ & $\begin{array}{l}\$ 0 \\
\$ 0\end{array}$ & $\begin{array}{r}\$ 11,475 \\
\$ 0\end{array}$ \\
\hline Markups & $0.00 \%$ & $\$ 0$ & $\$ 0$ & $\$ 0$ & $\$ 0$ & $\$ 0$ & $\$ 0$ \\
\hline $\begin{array}{l}\text { Subtotal Estimate } \\
\text { Escalation }\end{array}$ & & $\$ 1,064$ & so & so & so & so & $\begin{array}{r}\$ 11,475 \\
\$ 1,064\end{array}$ \\
\hline Management Reserve & & $\begin{array}{l}\$ 1,384 \\
\$ 4,389\end{array}$ & 幽 & 幽 & \$ & $\$ 0$ & $\begin{array}{l}\$ 1,004 \\
\$ 4,389\end{array}$ \\
\hline $\begin{array}{l}\text { Total } 2.5 \text { Perform Sub } \\
\text { Excav'ns, Floor }\end{array}$ & & $\$ 16,928$ & \$o & \$o & $\$ 0$ & \$o & $\$ 16,928$ \\
\hline
\end{tabular}


Project Name: RTC Waste Heat Recovery

Project Location: ATR

Estimate Number: 7850

\section{Code Description contractor}

$2.6 \mathrm{~cm} /$ Support During Design ana Closeour

Memo: Allowance based on estimator judgment.

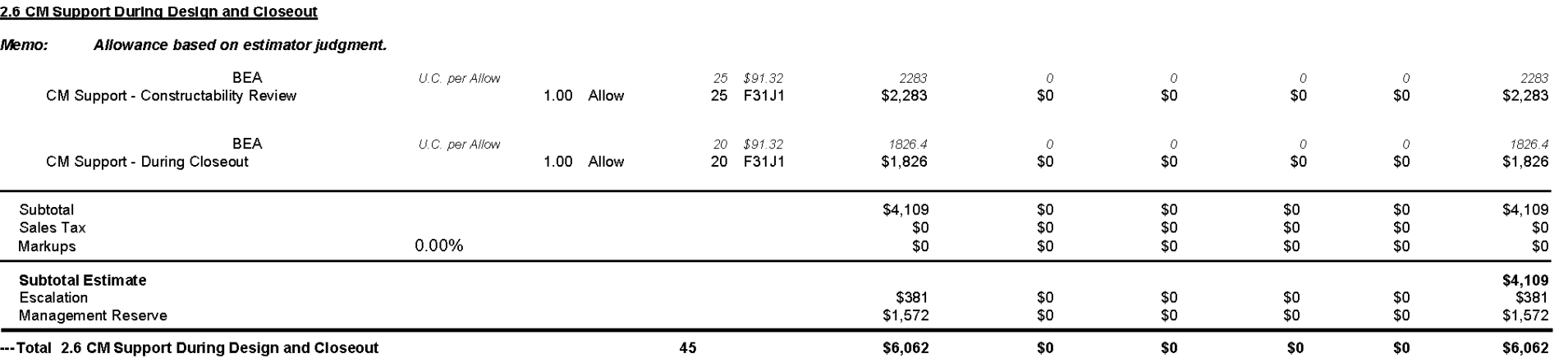

3.1.1 BEA Engineering \& Design Conceptual Phase

Memo: Based on estimator judgment.

BEA
E34W2
PROJECT ENGINEER (1/4 Time Coverage)

E04W1 ${ }_{\text {CIIL/STRUCTURAL ENGR }}^{\text {BEA }}$

EEA
E11W1
MECHANICAL ENGINEERING

E41W1

EO6W1
ELECTRICAL ENGINEERING

${ }^{\text {E19H1 }}$ SAFETY ENGINEERING

BEA

5/11/2012 11:08:15
DETAIL ITEM REPORT

Client: C. P.Ischay

Prepared By: A. W. Miller/S. N. Wasley
Estimate Type: Class-5

Qty UOM Hrs Resource Labor Equipment Material Subcontractor Other TOTAL

\begin{tabular}{|c|c|c|c|c|c|c|c|c|c|c|}
\hline UC. perWts & 8.00 & Wks & $\begin{array}{l}10 \\
80\end{array}$ & $\begin{array}{l}\$ 133.25 \\
\text { E34W }\end{array}$ & $\begin{array}{r}1332.5 \\
\$ 10,660\end{array}$ & \$o & \$o & \$o & $\$ 0$ & $\begin{array}{r}1332.5 \\
\$ 10,660\end{array}$ \\
\hline Uo, perwhs & 8.00 & Wks & 240 & $\begin{array}{l}\text { S148.5日 } \\
\text { E04W1 }\end{array}$ & $\begin{array}{r}4457.44 \\
\$ 35,659\end{array}$ & $\$ 0$ & $\$ 0$ & $\$ 0$ & $\$ 0$ & $\begin{array}{l}4457.4 \\
\$ 35,659\end{array}$ \\
\hline UC perwks & 8.00 & Wks & $\begin{array}{r}310 \\
210\end{array}$ & 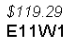 & $\begin{array}{r}3578,7 \\
\$ 28,630\end{array}$ & \$o & $\$ 0$ & $\$ 0$ & $\$ 0$ & $\begin{array}{r}3578.7 \\
\$ 28,630\end{array}$ \\
\hline UC. perwas & 8.00 & Wks & 80 & $\begin{array}{l}5126.49 \\
\text { E41WW }\end{array}$ & $\begin{array}{r}1264.9 \\
\$ 10,119\end{array}$ & $\$ 0$ & $\$ 0$ & $\begin{array}{l}0 \\
\text { \$o }\end{array}$ & \$0 & $\begin{array}{r}12649 \\
\$ 10,119\end{array}$ \\
\hline U. perwts & 8.00 & Wks & $\begin{array}{r}20 \\
160\end{array}$ & $\begin{array}{l}\$ 118.73 \\
\text { EDow1 }\end{array}$ & $\begin{array}{r}2374.6 \\
\$ 18,997\end{array}$ & $\$ 0$ & $\$ 0$ & $\$ 0$ & $\$ 0$ & $\begin{array}{r}23746 \\
\$ 18,997\end{array}$ \\
\hline U. per $w$ ks & 8.00 & Wks & 80 & $\begin{array}{l}\$ 114.99 \\
\text { E19H1 }\end{array}$ & $\begin{array}{r}\$ 149.9 \\
\$ 9,199\end{array}$ & $\$ 0$ & $\$ 0$ & $\$ 0$ & $\$ 0$ & $\begin{array}{r}1149.9 \\
\$ 9,199\end{array}$ \\
\hline
\end{tabular}


Project Name: RTC Waste Heat Recovery

Project Location: ATR

\section{Code Description Contractor}

3.1.1 BEA Engineering \& Desian conceptual Pnase

Memo: $\quad$ Based on estimator judgmem.

EESA
INSTRUMENTATION AND CONTROLS

EO5W1 BEA

T03J1 BEA

E32W1 ${ }_{\text {SYSTEM/SSC ENGINEER }}^{\text {BEA }}$
E41W1 FIRE PROTECTION BEA

E35W1
INSTRUMENTATION AND CONTROLS

EOBW2
ENVIRONMENTAL ENGINEERING

EO5W1 BEA

QUALITY ENGINEERING BEA

E19H1 ${ }_{\text {SAFETY ENGINEERING }}$
DETAIL ITEM REPORT

Client: C.P.Ischay

Prepared By: A. W. Miller/S. N. Wasle

Estimate Type: Class-5

Qty UOM

Hrs Resource Labor

Equipment

Material Subcontractor

Other

TOTAL

\begin{tabular}{|c|c|c|c|c|c|c|c|c|c|c|}
\hline U.C. perWks & 8.00 & Wks & $\begin{array}{l}10 \\
80\end{array}$ & 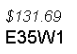 & $\begin{array}{r}1316.9 \\
\$ 10,535\end{array}$ & $\begin{array}{l}0 \\
\text { so }\end{array}$ & $\begin{array}{l}0 \\
\$ 0\end{array}$ & $\begin{array}{r}0 \\
\$ 0\end{array}$ & $\$ 0$ & $\begin{array}{r}1316.9 \\
\$ 10,535\end{array}$ \\
\hline UC. per Whs & 8.00 & Wks & $\begin{array}{c}10 \\
80\end{array}$ & $\begin{array}{l}590.26 \\
\text { E05W1 }\end{array}$ & $\begin{array}{r}902.6 \\
\$ 7,221\end{array}$ & $\begin{array}{l}0 \\
\$ 0\end{array}$ & $\begin{array}{l}0 \\
\$ 0\end{array}$ & $\begin{array}{l}0 \\
\$ 0\end{array}$ & $\$ 0$ & $\begin{array}{l}902.6 \\
\$ 7,221\end{array}$ \\
\hline UC. per Whs & 8.00 & Wks & $\begin{array}{r}40 \\
320\end{array}$ & $\begin{array}{l}568.02 \\
\text { T03W1 }\end{array}$ & $\begin{array}{r}2720.8 \\
\$ 21,766\end{array}$ & $\begin{array}{l}0 \\
\$ 0\end{array}$ & $\begin{array}{r}0 \\
\$ 0\end{array}$ & $\begin{array}{l}0 \\
\$ 0\end{array}$ & \$o & $\begin{array}{r}2720.8 \\
\$ 21,766\end{array}$ \\
\hline UC. per Reviews & 1.00 & Reviews & $\begin{array}{l}20 \\
20\end{array}$ & $\begin{array}{l}\$ 121.94 \\
\text { E32W1 }\end{array}$ & $\begin{array}{r}2438.8 \\
\$ 2,439\end{array}$ & $\begin{array}{l}0 \\
\text { so }\end{array}$ & $\begin{array}{l}0 \\
\text { so }\end{array}$ & so & \$o & $\begin{array}{r}2438.8 \\
\$ 2,439\end{array}$ \\
\hline U.c. per Reviews & 1.00 & Reviews & $\begin{array}{l}10 \\
10\end{array}$ & $\begin{array}{l}\$ 126.49 \\
\text { E41W1 }\end{array}$ & $\begin{array}{r}1264.9 \\
\$ 1,265\end{array}$ & $\begin{array}{l}0 \\
\text { so }\end{array}$ & $\begin{array}{r}0 \\
\text { so }\end{array}$ & $\begin{array}{r}0 \\
\$ 0\end{array}$ & $\begin{array}{l}0 \\
\text { \$o }\end{array}$ & $\begin{array}{r}1264.9 \\
\$ 1,265\end{array}$ \\
\hline Uc. per Reviews & 1.00 & Reviews & $\begin{array}{l}10 \\
10\end{array}$ & $\begin{array}{l}\$ 131.69 \\
\text { E35WW1 }\end{array}$ & $\begin{array}{r}1316.9 \\
\$ 1,317\end{array}$ & \$o & $\begin{array}{l}0 \\
\$ 0\end{array}$ & $\begin{array}{r}0 \\
\$ 0\end{array}$ & \$o & $\begin{array}{r}1316.9 \\
\$ 1,317\end{array}$ \\
\hline Uc. per Reviews & 1.00 & Reviews & $\begin{array}{l}6 \\
6\end{array}$ & $\begin{array}{l}\$ 131.59 \\
\text { E08w1 }\end{array}$ & $\begin{array}{r}789.54 \\
\$ 790\end{array}$ & $\$ 0$ & $\begin{array}{l}0 \\
\$ 0\end{array}$ & $\$$ & $\$ 0$ & $\begin{array}{r}789.54 \\
\$ 790\end{array}$ \\
\hline U.c. per Reviews & 1.00 & Reviews & $\begin{array}{l}10 \\
10\end{array}$ & $\begin{array}{l}\$ 90.26 \\
\text { E05w1 }\end{array}$ & $\begin{array}{l}902.6 \\
\$ 903\end{array}$ & $\begin{array}{l}0 \\
\$ 0\end{array}$ & $\begin{array}{r}0 \\
\$ 0\end{array}$ & $\begin{array}{c}0 \\
\$ 0\end{array}$ & $\begin{array}{c}0 \\
\$ 0\end{array}$ & $\begin{array}{l}902.6 \\
\$ 903\end{array}$ \\
\hline UC. per Reviews & 1.00 & Reviews & $\begin{array}{l}20 \\
20\end{array}$ & $\begin{array}{l}\$ 111.17 \\
\text { E17WW5 }\end{array}$ & $\begin{array}{r}2223.4 \\
\$ 2,223\end{array}$ & $\begin{array}{l}0 \\
\$ 0\end{array}$ & $\begin{array}{l}0 \\
\$ 0\end{array}$ & $\begin{array}{l}0 \\
\$ 0\end{array}$ & \$0 & $\begin{array}{r}2223.4 \\
\$ 2,223\end{array}$ \\
\hline U. per Reviews & 1.00 & Reviews & $\begin{array}{l}10 \\
10\end{array}$ & $\begin{array}{l}\$ 114.99 \\
\text { E19H1 }\end{array}$ & $\begin{array}{r}1149.9 \\
\$ 1,150\end{array}$ & $\begin{array}{l}0 \\
\$ 0\end{array}$ & $\begin{array}{r}0 \\
\text { \$0 }\end{array}$ & $\begin{array}{r}0 \\
\$ 0\end{array}$ & \$o & $\begin{array}{r}1149.9 \\
\$ 1,150\end{array}$ \\
\hline
\end{tabular}

BEA

5/11/2012 11:08:15 
Project Name: RTC Waste Heat Recovery

Project Location: ATR

\section{Code Description Contractor}

3.1.1 BEA Engineering \& Desian conceptual Pnase

Memo: Based on estimator judgment.

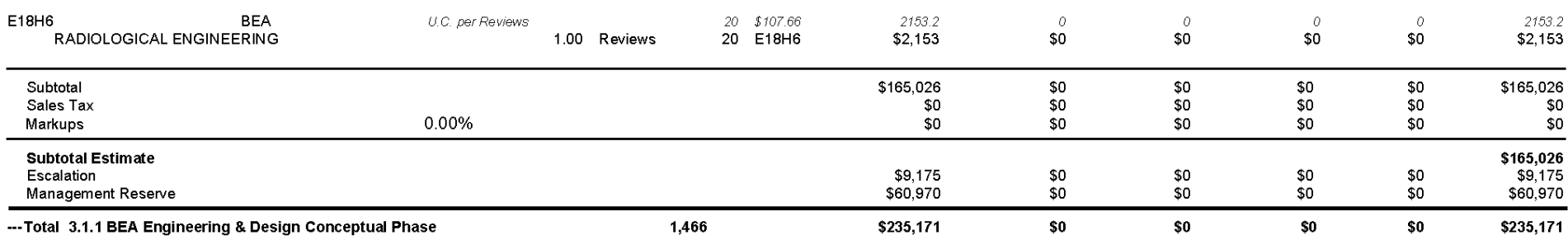

నิ

\subsubsection{EEA Engineering \& Design Preliminary Phase}

Memo: Based on estimator judgment.

EEA
PRAW2
PROJECT ENGINEER (1/4 Time Coverage)

E04W1 ${ }_{\text {CIVIL/STRUCTURAL ENGR }}^{\text {BEA }}$

BEA
E11W1
MECHANICAL ENGINEERING

E41W1

EEA
ELEW1

E19H1 SAFETY ENGINEERING BEA

E35W1
INSTRUMENTATION AND CONTROLS

u.c. perwas

$6.00 \mathrm{Wks}$

$\begin{array}{ll}10 & \$ 133.25 \\ 60 & \text { E34W2 }\end{array}$

1332.5
$\$ 7,995$

$\begin{array}{rr}30 & \$ 148.58 \\ 180 & \text { E04W1 }\end{array}$

6.00 Wks

U. perwks

6.00 Wks

$\begin{array}{rr}30 & \$ 119.29 \\ 180 & \text { E11W1 }\end{array}$

$\$ 26,744$

3579.7
$\$ 21,472$

uc. per Whs

$6.00 \quad$ Wke

$\begin{array}{cc}10 & \$ 126.49 \\ 60 & \text { E11W1 }\end{array}$

u.c. perwhs

6.00 Wks

$\begin{array}{rl}20 & 511879 \\ 120 \\ \text { E006w1 }\end{array}$

$\$ 7,589$

U.c. perwhs

6.00 Wks

$\begin{array}{ll}10 & \$ 114.99 \\ 60 & \mathrm{E} 19 \mathrm{H} 1\end{array}$

$\$ 14,248$

U.C. per Whs

6.00 Wks

$\begin{array}{ll}10 & \$ 131.69 \\ 60 & \text { E35W1 }\end{array}$

$\$ \$ 149.9$

1316.9
$\$ 7,901$

Cost Estimating

Client: C.P.Ischay

Prepared By: A.W. Miller/S. N. Wasle
Estimate Type: Class-5

Equipment Material Subcontractor Other TOTAL

BEA

11:08:15

\begin{tabular}{|c|c|c|c|c|}
\hline$\$ 0$ & $\begin{array}{l}0 \\
\$ 0\end{array}$ & $\begin{array}{l}0 \\
\$ 0\end{array}$ & $\$ 0$ & $\begin{array}{r}1332.5 \\
\$ 7,995\end{array}$ \\
\hline$\$ 0$ & $\begin{array}{l}0 \\
\$ 0\end{array}$ & $\begin{array}{l}0 \\
\$ 0\end{array}$ & $\$ 0$ & $\begin{array}{r}4457.4 \\
\$ 26,744\end{array}$ \\
\hline$\$ 0$ & $\$ 0$ & \$o & $\$ 0$ & $\begin{array}{r}3578.7 \\
\$ 21,472\end{array}$ \\
\hline$\$ 0$ & \$o & $\begin{array}{r}0 \\
\$ 0\end{array}$ & $\$ 0$ & $\begin{array}{r}1264.9 \\
\$ 7,589\end{array}$ \\
\hline$\$ 0$ & \$o & $\begin{array}{r}0 \\
\$ 0\end{array}$ & $\$ 0$ & $\begin{array}{r}2374,6 \\
\$ 14,248\end{array}$ \\
\hline & \$0 & $\$ 0$ & $\$ 0$ & $\begin{array}{r}1749.9 \\
\$ 6,899\end{array}$ \\
\hline & $\$ 0$ & \$o & $\$ 0$ & $\begin{array}{r}1316.9 \\
\$ 7,901\end{array}$ \\
\hline
\end{tabular}


Project Name: RTC Waste Heat Recovery

Project Location: ATR

\section{Code Description Contractor}

3.1.2 BEA Engineering \& Design Prellminary Pnase

Memo: Based on estimator judgment.

T03.1 BEA

E32W1 ${ }_{\text {SYSTEM/SSC ENGINEER }}^{\text {BEA }}$

E41W1

TRE PROTECTION

EEA
E35W1
INSTRUMENTATION AND CONTROLS

BEA
EOBW2
ENVIRONMENTAL ENGINEERING

E05W1 BEA

QUALITY ENGINEERING BEA

E19H1 1 SAFETY ENGINEERING BEA

\section{DETAIL ITEM REPORT}

aty UOM

Hrs Resource Labor

TOTAL

\begin{tabular}{|c|c|c|c|c|c|c|c|c|c|c|}
\hline U. perWhs & 6.00 & Wks & $\begin{array}{l}10 \\
60\end{array}$ & $\begin{array}{l}590.26 \\
\text { E05W } 1\end{array}$ & $\begin{array}{r}902.6 \\
\$ 5,416\end{array}$ & $\$ 0$ & $\$ 0$ & $\begin{array}{l}0 \\
\$ 0\end{array}$ & $\$ 0$ & $\begin{array}{r}902,6 \\
\$ 5,416\end{array}$ \\
\hline UC. perWhs & 6.00 & Wks & $\begin{array}{r}40 \\
240\end{array}$ & $\begin{array}{l}568.02 \\
\text { T03W1 }\end{array}$ & $\begin{array}{r}2720.8 \\
\$ 16,325\end{array}$ & $\$ 0$ & $\begin{array}{l}0 \\
\$ 0\end{array}$ & $\begin{array}{l}0 \\
\$ 0\end{array}$ & \$0 & $\$ 16,325$ \\
\hline uc. per Reviews & 1.00 & Reviews & $\begin{array}{l}20 \\
20\end{array}$ & $\begin{array}{l}\$ 121.94 \\
\text { E32W1 }\end{array}$ & $\begin{array}{r}2438.8 \\
\$ 2,439\end{array}$ & $\begin{array}{r}0 \\
\$ 0\end{array}$ & $\begin{array}{r}0 \\
\$ 0\end{array}$ & $\begin{array}{r}0 \\
\$ 0\end{array}$ & $\begin{array}{l}0 \\
\$ 0\end{array}$ & $\begin{array}{r}2438.8 \\
\$ 2,439\end{array}$ \\
\hline UC. per Reviews & 1.00 & Reviews & $\begin{array}{l}10 \\
10\end{array}$ & $\begin{array}{l}\$ 126.49 \\
\text { E41W1 }\end{array}$ & $\begin{array}{r}1264.9 \\
\$ 1,265\end{array}$ & $\begin{array}{l}0 \\
\$ 0\end{array}$ & $\begin{array}{l}0 \\
\$ 0\end{array}$ & $\begin{array}{l}0 \\
\$ 0\end{array}$ & $\$ 0$ & $\begin{array}{r}1264.9 \\
\$ 1,265\end{array}$ \\
\hline U.C. per Reviews & 1.00 & Reviews & $\begin{array}{l}10 \\
10\end{array}$ & $\begin{array}{l}\$ 131.69 \\
\text { E35W1 }\end{array}$ & $\begin{array}{r}1316.9 \\
\$ 1,317\end{array}$ & so & \$o & $\begin{array}{l}0 \\
\$ 0\end{array}$ & \$o & $\begin{array}{r}1316.9 \\
\$ 1,317\end{array}$ \\
\hline Uc. per Reviews & 1.00 & Reviews & $\begin{array}{l}6 \\
6\end{array}$ & $\begin{array}{l}\$ 131.59 \\
\text { E08WW2 }\end{array}$ & $\begin{array}{r}789.54 \\
\$ 790\end{array}$ & $\begin{array}{l}0 \\
\$ 0\end{array}$ & $\begin{array}{l}0 \\
\$ 0\end{array}$ & $\begin{array}{l}0 \\
\$ 0\end{array}$ & $\begin{array}{l}0 \\
\$ 0\end{array}$ & $\begin{array}{r}789.54 \\
\$ 790\end{array}$ \\
\hline U.c. per Reviews & 1.00 & Reviews & $\begin{array}{l}10 \\
10\end{array}$ & $\begin{array}{l}\$ 90.26 \\
\text { E05W1 }\end{array}$ & $\begin{array}{l}902.6 \\
\$ 903\end{array}$ & $\$ 0$ & $\$ 0$ & $\$ 0$ & $\$ 0$ & $\begin{array}{l}902.6 \\
\$ 903\end{array}$ \\
\hline UC. per Reviews & 1.00 & Reviews & $\begin{array}{l}20 \\
20\end{array}$ & $\begin{array}{l}\$ 111.17 \\
\text { E17WW5 }\end{array}$ & $\begin{array}{r}2223.4 \\
\$ 2,223\end{array}$ & $\begin{array}{l}0 \\
\$ 0\end{array}$ & $\begin{array}{l}0 \\
\$ 0\end{array}$ & $\begin{array}{l}0 \\
\$ 0\end{array}$ & $\begin{array}{l}0 \\
\$ 0\end{array}$ & $\begin{array}{r}2223.4 \\
\$ 2,223\end{array}$ \\
\hline UC. per Reviews & 1.00 & Reviews & $\begin{array}{l}10 \\
10\end{array}$ & $\begin{array}{l}\$ 114.99 \\
\text { E19H1 }\end{array}$ & $\begin{array}{r}1149.9 \\
\$ 1,150\end{array}$ & $\begin{array}{l}0 \\
\$ 0\end{array}$ & $\$ 0$ & $\stackrel{0}{\$ 0}$ & $\begin{array}{l}0 \\
\$ 0\end{array}$ & $\begin{array}{r}\$ 149.9 \\
\$ 1,150\end{array}$ \\
\hline
\end{tabular}

Equipment

Material Subcontractor

Othe

Client: C. P. Ischay

Prepared By: A. W. Miller/S. N. Wasle
Estimate Type:
Class-5

$$
\begin{aligned}
& \begin{array}{l}
9954 \\
5790
\end{array} \\
& \begin{array}{r}
902.6 \\
5903
\end{array} \\
& 223
\end{aligned}
$$

BEA

5/11/2012 11:08:15 1149.9
$\$ 1,150$ 
Project Name: RTC Waste Heat Recovery

Project Location: ATR

\section{Code Description Contractor}

3.1.2 BEA Engineering \& Design Prellminary Pnase

Memo: Based on estimator judgment.

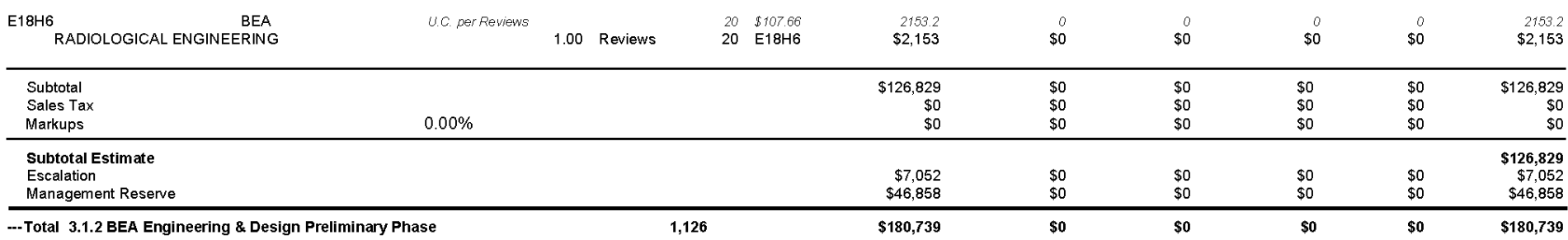

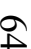

\subsubsection{BEA Engineering \& Design Final Phase}

Memo: $\quad$ Based on estimator judgment.

EEAW2
PROJECT ENGINEER (1/4 Time Covera

${ }_{\text {CIILISTRUCTURAL ENGR }}^{\text {BEA }}$

EEA
E11W1
MECHANICAL ENGINEERING

E41W1

EEA
ELEW1

E19H1 SAFETY ENGINEERING BEA

E35W1
INSTRUMENTATION AND CONTROLS

BEA

5/11/2012 11:08:15
DETAIL ITEM REPORT

Client: C.P.Ischay

Prepared By: A.W. Miller/S. N. Wasley

Estimate Type: Class-5

Qty UOM Hrs Resource Labor Equipment Material Subcontractor Other TOTAL

1,126

U.c. perwks

10.00 Wks

U. per Whs

10.00 Wke

$\begin{array}{rr}10 & \$ 133.25 \\ 100 & \mathrm{E} 34 \mathrm{~W} 2\end{array}$

$\$ 1332.5$

0
$\$ 0$

0
$\$ 0$

$\$ 0$

$\$ 0$

$\begin{array}{rr}30 & \$ 148.58 \\ 300 & \text { E04W1 }\end{array}$

4457.4
$\$ 44,574$

0
$\$ 0$

$\begin{array}{rr}30 & \$ 119.29 \\ 300 & \text { E11W1 }\end{array}$

3570.7
$\$ 35,787$

$\begin{array}{rl}10 & 5126.49 \\ 100 & \text { E11W1 }\end{array}$

12649
$\$ 12,619$

U.C. per WHS

10.00 Wks

$\begin{array}{rr}20 & \$ 118.73 \\ 200 & \text { E06W1 }\end{array}$

2374.6
$\$ 23,746$

uc. perwhs

10.00 Wks

$\begin{array}{rr}10 & \$ 114.99 \\ 100 & \mathrm{E} 19 \mathrm{H} 1\end{array}$

1149.9
$\$ 11,499$

$\begin{array}{rr}10 & \$ 131.69 \\ 100 & \text { E35W1 }\end{array}$

1316.9
$\$ 13,169$

10.00 Wks

Cost Estimating Page No. 14
Material costs where applicable include Idaho State Sales Tax 
Project Name: RTC Waste Heat Recovery

Project Location: ATR

\section{Code Description Contractor}

3.1.3 BEA Enquneering \& Desian Final Pnase

Memo: Based on estimator judgment.

E05W1 BEA

T03J1 BEA

E32W1 ${ }_{\text {SYSTEM/SSC ENGINEER }}^{\text {BEA }}$

E41W1 BEA

FIRE PROTECTION

E35W1
INSTRUMENTATION AND CONTROLS

BEA
ENVIRONMENTAL ENGINEERING

E05W1 BEA

7W5 QUALITY ENGINEERING BEA

E19H1 1 SAFETY ENGINEERING BEA

\section{DETAIL ITEM REPORT}

Qty

UOM

Hrs Resource Labor

10.00 Wks

$\begin{aligned} 10 & \$ 90.26 \\ 100 & \text { E05W1 }\end{aligned}$

$\begin{array}{rr}40 & \$ 68.02 \\ 400 & \text { T03W1 }\end{array}$

4.c. perWhs 10.00 Wks

uc. per Reviews

1.00 Reviews

$\begin{array}{ll}20 & \$ 121.94 \\ 20 & \text { E32W1 }\end{array}$

$\begin{array}{cc}10 & \$ 126.49 \\ 10 & \text { E41W1 }\end{array}$

1.00 Reviews

4.C. per Reviews

1.00 Reviews

$\begin{array}{ll}10 & \$ 131.69 \\ 10 & \text { E35W1 }\end{array}$

$\begin{array}{ll}6 & \$ 131.59 \\ 6 & \text { E08W2 }\end{array}$

1.00 Reviews

10.90020

1.00 Reviews

$\begin{aligned} 10 & \$ 90.26 \\ 10 & \text { E05W1 }\end{aligned}$

$\begin{array}{ll}20 & \$ 111.17 \\ 20 & \text { E17W5 }\end{array}$

1.00 Reviews

1.00 Reviews
Equipment

Material Subcontractor

Other

TOTAL

9026
$\$ 9020$

$\$ 2720.8$

Client:

C.P. Ischay

Prepared By: A. W. Miller/S. N. Wasley

stimate Type: Class-5

Other

902.6
$\$ 9,026$

2720.8
$\$ 27,208$

2438.8
$\$ 2,439$

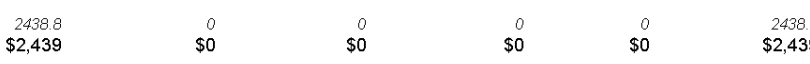

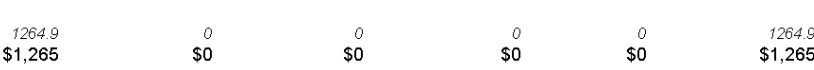

$\$ 1,31$

$\mathbf{7 8 9 . 5 4}$

902.

$\$ 902.6$

2223.4
$\$ 2,223$

$\$ 1,150$
BEA

11:08:15 
Project Name: RTC Waste Heat Recovery

Project Location: ATR

Code Description $\quad$ Contractor 3.1.3 BEA Enquneering \& Desian Final Pnase

Memo: Based on estimator judgment.

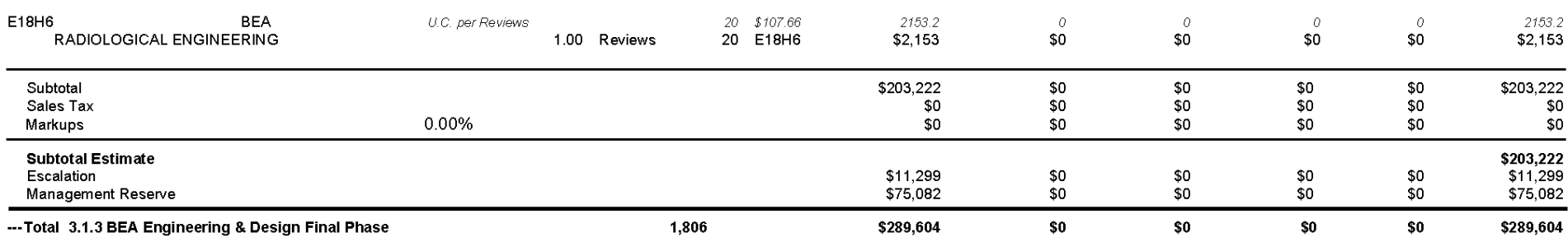

4.1 Environmental Documents (NEPA Checklist)

Memo: Allowance to prepare the environmental checklist documentation for the project, estimator's judgment

\begin{tabular}{|c|c|c|c|c|c|c|c|c|c|}
\hline $\begin{array}{l}\text { PEA } \\
\text { Prepare NEPA Documentation (Environmental } \\
\text { Checklist - EC) }\end{array}$ & U. per Hrs & $40.00 \mathrm{Hrs}$ & $\begin{array}{rr}1 & \$ 122.44 \\
40 & \$ 21 G \mathrm{C}\end{array}$ & $\begin{array}{r}122.44 \\
\$ 4,898\end{array}$ & $\$ 0$ & $\$ 0$ & $\begin{array}{l}0 \\
\$ 0\end{array}$ & $\$ 0$ & $\begin{array}{r}122,44 \\
\$ 4,898\end{array}$ \\
\hline $\begin{array}{l}\text { Subtotal } \\
\text { Sales Tax } \\
\text { Markups }\end{array}$ & $0.00 \%$ & & & $\begin{array}{r}\$ 4,898 \\
\$ 0 \\
\$ 0\end{array}$ & $\begin{array}{l}\$ 0 \\
\$ 0 \\
\$ 0\end{array}$ & $\begin{array}{l}\$ 0 \\
\$ 0 \\
\$ 0\end{array}$ & $\begin{array}{l}\$ 0 \\
\$ 0 \\
\$ 0\end{array}$ & $\begin{array}{l}\$ 0 \\
\$ 0 \\
\$ 0\end{array}$ & $\begin{array}{r}\$ 4,898 \\
\$ 0 \\
\$ 0\end{array}$ \\
\hline $\begin{array}{l}\text { Subtotal Estimate } \\
\text { Escalation } \\
\text { Management Reserve }\end{array}$ & & & & $\begin{array}{r}\$ 272 \\
\$ 1,809\end{array}$ & $\begin{array}{l}\$ 0 \\
\$ 0\end{array}$ & $\begin{array}{l}\$ 0 \\
\$ 0\end{array}$ & $\begin{array}{l}\$ 0 \\
\$ 0\end{array}$ & $\begin{array}{l}\$ 0 \\
\$ 0\end{array}$ & $\begin{array}{r}\$ 4,898 \\
\$ 272 \\
\$ 1,809\end{array}$ \\
\hline Total 4.1 Environmental D & & & & $\$ 6,9 / 9$ & $\$ 0$ & $\$ 0$ & so & $\$ 0$ & $\$ 6,9 / 9$ \\
\hline
\end{tabular}

\subsubsection{Prepare Fire Hazards Analysis Report}

Memo: Allowance to prepare hazards documents for the project,based on information provided by BEA fire protection engineering

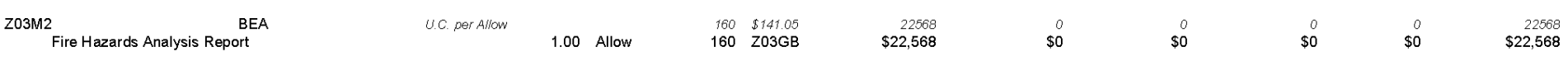


Project Name: RTC Waste Heat Recovery

Project Location: ATR

Pstimate Number: 7850

Code Description $\quad$ Contractor

4.2.1 Prepare Fire Hazards AnalysIS Repon

Memo: $\quad$ Allowance to prepare hazards documents for the project, based on information provided by BEA fire protection engineering

\begin{tabular}{|c|c|c|c|c|c|c|c|}
\hline $\begin{array}{l}\text { Subtotal } \\
\text { Sales Tax } \\
\text { Markups }\end{array}$ & $0.00 \%$ & $\begin{array}{r}\$ 22,568 \\
\$ 0 \\
\$ 0\end{array}$ & $\begin{array}{l}\$ 0 \\
\$ 0 \\
\$ 0\end{array}$ & $\begin{array}{l}\$ 0 \\
\$ 0 \\
\$ 0\end{array}$ & $\begin{array}{l}\$ 0 \\
\$ 0 \\
\$ 0\end{array}$ & $\begin{array}{l}\$ 0 \\
\$ 0 \\
\$ 0\end{array}$ & $\begin{array}{r}\$ 22,568 \\
\$ 0 \\
\$ 0\end{array}$ \\
\hline $\begin{array}{l}\text { Subtotal Estimate } \\
\text { Escalation } \\
\text { Management Reserve }\end{array}$ & & $\begin{array}{l}\$ 1,255 \\
\$ 8,338\end{array}$ & $\begin{array}{l}\$ 0 \\
\$ 0\end{array}$ & $\begin{array}{l}\$ 0 \\
\$ 0\end{array}$ & $\begin{array}{l}\$ 0 \\
\$ 0\end{array}$ & $\$ 0$ & $\begin{array}{r}\$ 22,568 \\
\$ 1,255 \\
\$ 8,338\end{array}$ \\
\hline
\end{tabular}

4.3.1 Prepare Davis Bacon Determination Documents

Memo: Allowance for Davis Bacon Determination

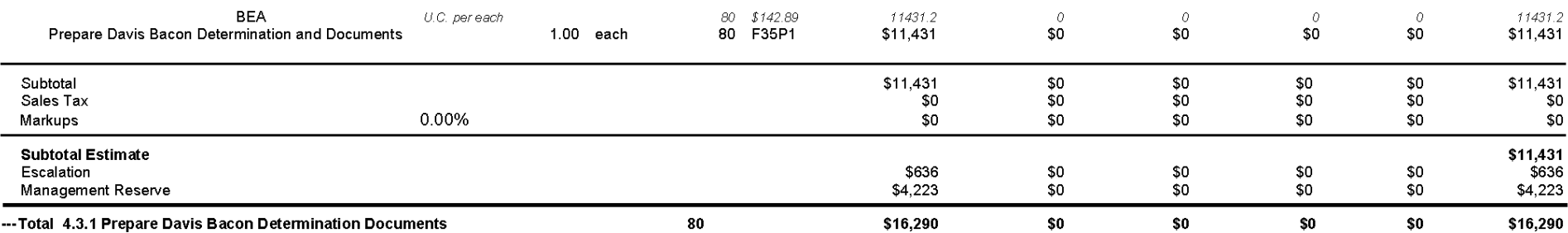

4.4 Prepare Unreviewed Safetv Questions (USQS

Memo: Based on discussion with SMEs with prior experience working at the ATR facility.
Prepared USQ Documentation
U.c. per EA
6.00 EA
$\begin{array}{llr}120 & \$ 119.29 & \$ 4314.8 \\ 720 & \text { E11W1 } & \$ 85,889\end{array}$
$\$ 0$
$\$ 0$
$\$ 0$
14314.8
$\$ 85,889$

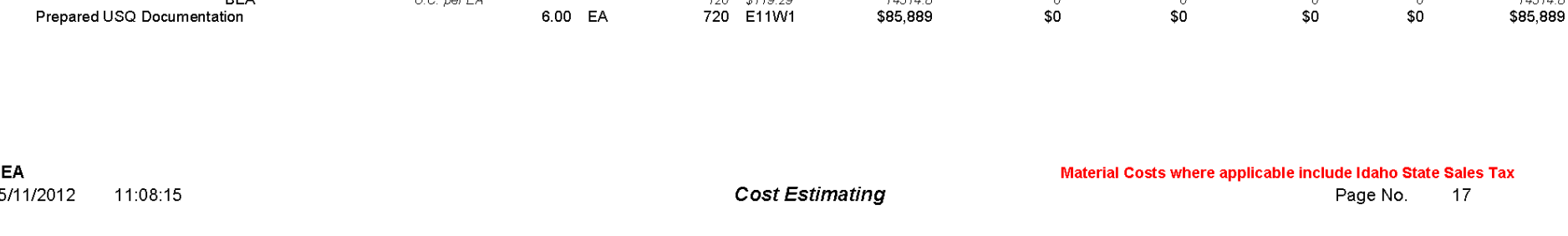


Project Name: RTC Waste Heat Recovery

Project Location: ATR

stimate Number: 7850

Code Description $\quad$ Contractor

4.4 Prepare Unrevlewed Saretv Questions (USQs

Memo: $\quad$ Based on discussion with SMEs with prior experience working at the ATR facility.

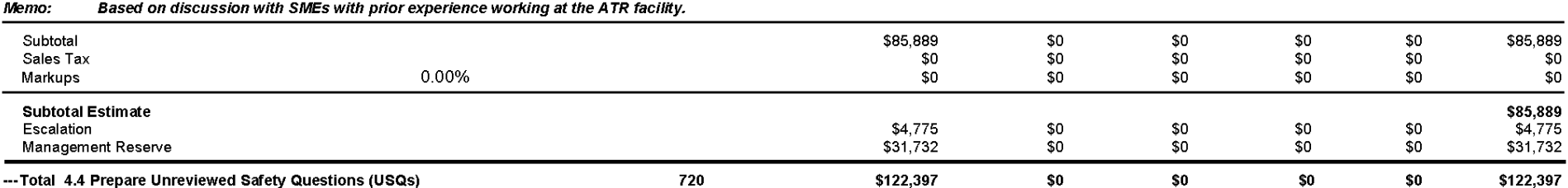

4.5.1 Probabilistic Risk Assessment (PRA) Preliminary

Client: C.P.Ischay

Prepared By: A. W. Miller/S. N. Wasley

Subcontractor Other TOTAL

\section{\$0}

$\$ 4,775$

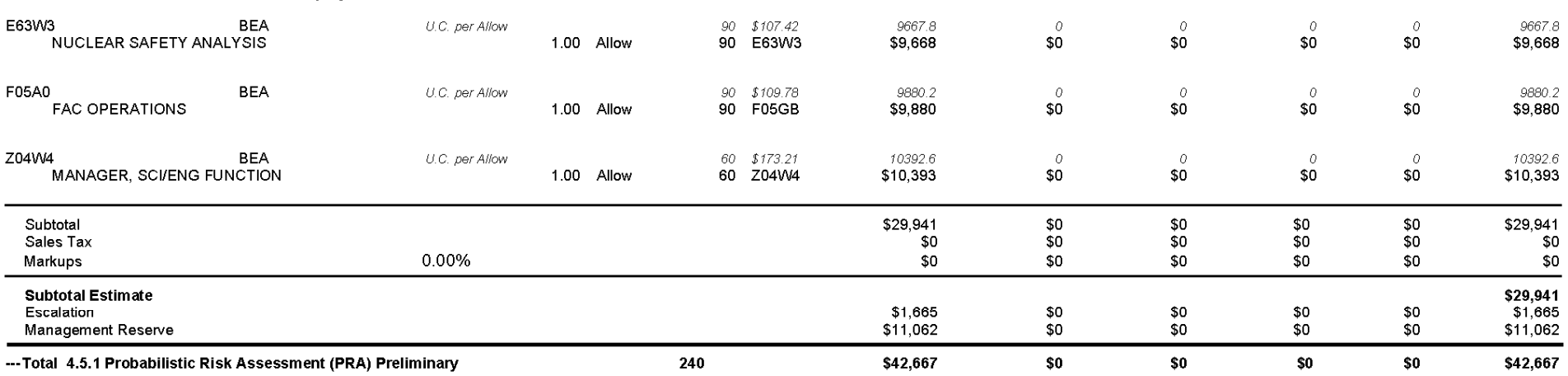

BEA

\section{DETAIL ITEM REPORT}

$\$ 42,667$

Cost Estimating

Material Costs where applicable include Idaho State Sales Tax

Page No. $\quad 18$ 
Project Name: RTC Waste Heat Recovery

Project Location: ATR

\section{Code Description $\quad$ contractor} 4.5.Z ZrobabullstIC RISK Assessment (PRA) Fina

Memo: Based on discussion with the project team

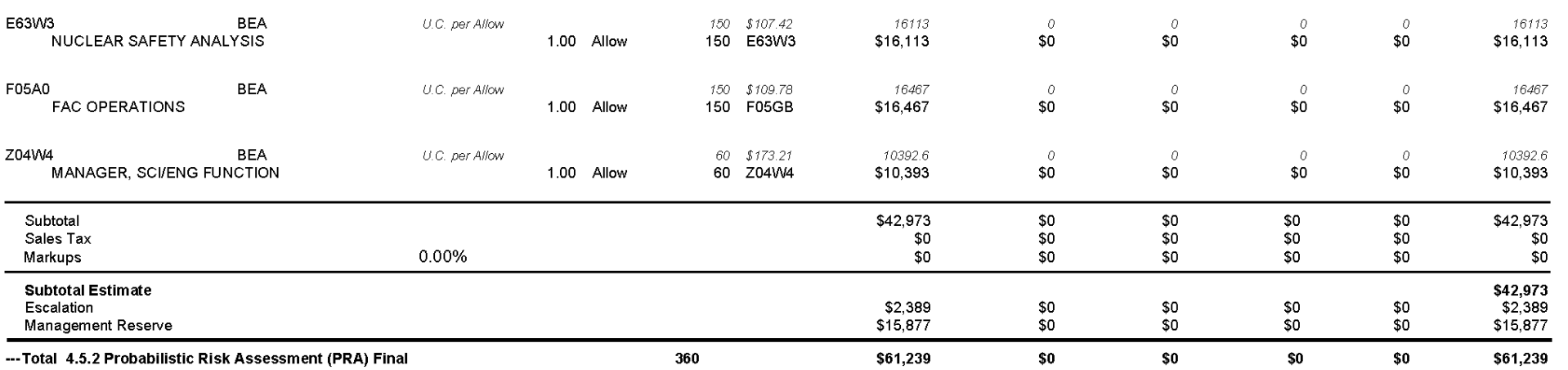

\subsubsection{General Contractor}

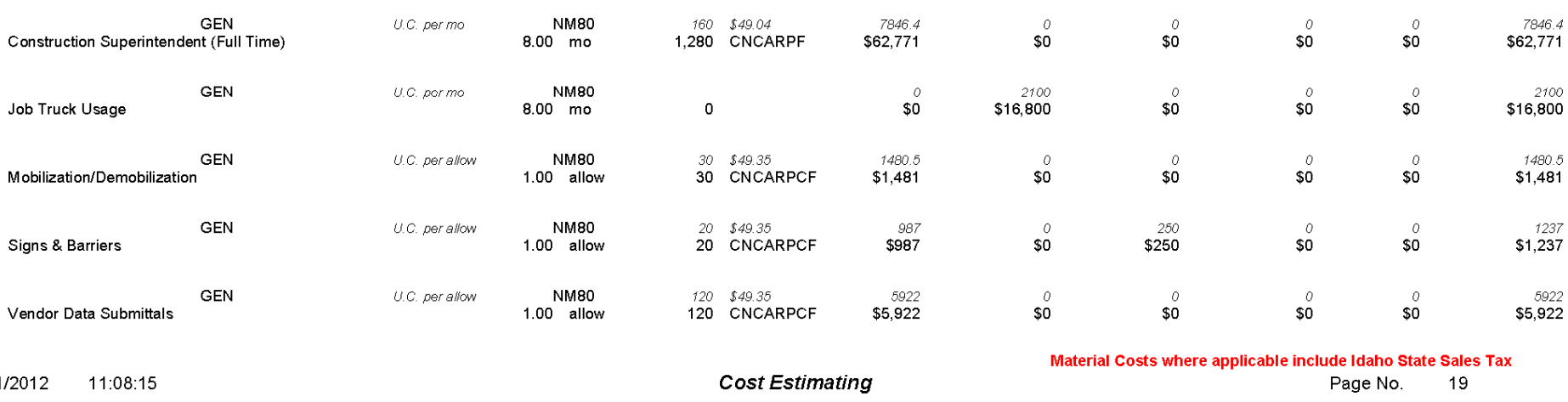

\section{DETAIL ITEM REPORT}

C.P.Ischay

Prepared By: A.W.Miller/S. N. Wasley

Estimate Type: Class-5

Qty UOM Hrs Resource Labor Equipment Material Subcontractor Other TOTAL
61,239 
Project Name: RTC Waste Heat Recovery

Project Location: ATR

Estimate Number: 7850

Code Description

5.1.1.1 General Contractor

Memo: Based on estmat

\begin{tabular}{|c|c|c|c|c|c|c|c|c|c|}
\hline $\begin{array}{c}G E N \\
\text { Equipment Usage (Lifts, Cranes, etc) }\end{array}$ & uc. permo & $1.00 \begin{array}{r}\mathrm{NM} 80 \\
\mathrm{mo}\end{array}$ & 0 & $\$ 0$ & $\begin{array}{r}18000 \\
\$ 18,000\end{array}$ & $\$ 0$ & $\begin{array}{l}0 \\
\$ 0\end{array}$ & $\$ 0$ & $\begin{array}{r}\quad 80000 \\
\$ 18,000\end{array}$ \\
\hline $\begin{array}{l}\text { Subtotal } \\
\text { Sales Tax } \\
\text { Markups }\end{array}$ & $35.76 \%$ & & & $\begin{array}{r}\$ 71,161 \\
\$ 0 \\
\$ 25,448 \\
\end{array}$ & $\begin{array}{r}\$ 34,800 \\
\$ 0 \\
\$ 12,445 \\
\end{array}$ & $\begin{array}{r}\$ 250 \\
\$ 15 \\
\$ 95 \\
\end{array}$ & $\begin{array}{l}\$ 0 \\
\$ 0 \\
\$ 0\end{array}$ & $\begin{array}{l}\$ 0 \\
\$ 0 \\
\$ 0\end{array}$ & $\begin{array}{r}\$ 106,211 \\
\$ 15 \\
\$ 37,988 \\
\end{array}$ \\
\hline $\begin{array}{l}\text { Subtotal Estimate } \\
\text { Escalation } \\
\text { Management Reserve }\end{array}$ & & & & $\begin{array}{r}\$ 8,956 \\
\$ 36,948\end{array}$ & $\begin{array}{r}\$ 4,380 \\
\$ 18,069\end{array}$ & $\begin{array}{r}\$ 33 \\
\$ 138\end{array}$ & $\begin{array}{l}\$ 0 \\
\$ 0\end{array}$ & $\begin{array}{l}\$ 0 \\
\$ 0\end{array}$ & $\begin{array}{r}\$ 144,214 \\
\$ 13,369 \\
\$ 55,154\end{array}$ \\
\hline 5.1.1.1 General Con & & & & $\$ 142,513$ & $\$ 69,693$ & $\$ 531$ & $\$ 0$ & $\$ 0$ & $\$ 212,737$ \\
\hline
\end{tabular}

\subsubsection{Piping Contractor}

Memo: Based on estimators judgment and the duration of the project.

\begin{tabular}{|c|c|c|c|c|c|c|c|c|c|c|}
\hline $\begin{array}{c}\text { MECH } \\
\text { Cratt Supenvision @ 20\% Admin Duties }\end{array}$ & U. per Months & $\begin{array}{l}\text { NM80 } \\
8.00 \text { Months }\end{array}$ & $\begin{array}{r}40 \\
320\end{array}$ & $\begin{array}{l}\text { s53.87 } \\
\text { CNPIPE }\end{array}$ & $\begin{array}{r}2154.8 \\
\$ 17,238\end{array}$ & $\$ 0$ & $\begin{array}{l}0 \\
\$ 0\end{array}$ & $\begin{array}{l}0 \\
\$ 0\end{array}$ & $\$$ & $\begin{array}{r}2154.8 \\
\$ 17,238\end{array}$ \\
\hline $\begin{array}{c}\text { MECH } \\
\text { Job Truck \& Equipment Usage Allowance }\end{array}$ & U.C. per Months & $\begin{array}{l}\text { NM80 } \\
8.00 \text { Months }\end{array}$ & 0 & & \$o & $\begin{array}{r}6.50 \\
\$ 5,200\end{array}$ & $\$ 0$ & $\$ 0$ & \$o & $\$ 5,200$ \\
\hline Vendor Data & UC. perallow & $\begin{array}{l}\text { NM80 } \\
1.00 \text { allow }\end{array}$ & $\begin{array}{l}40 \\
40\end{array}$ & $\begin{array}{l}\$ 53.87 \\
\text { CNPIPE }\end{array}$ & $\begin{array}{l}2154,8 \\
\$ 2,155\end{array}$ & $\$ 0$ & $\$ 200$ & $\$ 0$ & $\$ 0$ & $\$ 2,355$ \\
\hline Misc Testing & Uo porallow & $\begin{array}{c}\text { NM80 } \\
1.00 \stackrel{\text { allow }}{ }\end{array}$ & 480 & $\begin{array}{l}\text { CNPIPE } \\
\text { CNSB. }\end{array}$ & $\begin{array}{r}25857.6 \\
\$ 25,858\end{array}$ & $\$ 0$ & $\$ 2,000$ & $\$ 0$ & \$0 & $\$ 27,858$ \\
\hline Sign \& Barriers & UC. perallow & $\begin{array}{c}\text { NM80 } \\
1.00 \text { allow }\end{array}$ & $\begin{array}{l}80 \\
80\end{array}$ & $\begin{array}{l}\$ 53.87 \\
\text { CNPIPE }\end{array}$ & $\begin{array}{r}4309,6 \\
\$ 4,310\end{array}$ & $\$ 0$ & $\begin{array}{l}250 \\
\$ 250\end{array}$ & $\$ 0$ & \$o & $\begin{array}{r}4559,6 \\
\$ 4,560\end{array}$ \\
\hline Piping Labels & U.C. per Allow & $1.00 \stackrel{\text { NM80 }}{\text { Allow }}$ & $\begin{array}{l}40 \\
40\end{array}$ & $\begin{array}{l}\text { S55.87 } \\
\text { CNPIPE }\end{array}$ & $\begin{array}{l}2154.8 \\
\$ 2,155\end{array}$ & $\$ 0$ & $\begin{array}{r}1000 \\
\$ 1,000\end{array}$ & $\$ 0$ & \$o & $\begin{array}{r}3154,8 \\
\$ 3,155\end{array}$ \\
\hline
\end{tabular}


Project Name: RTC Waste Heat Recovery

Project Location: ATR

Primate Number: 7850

Code Description $\quad$ Contractor

5.1.1.2 Piping Contractor

Memo: Based on estimators judgment and the duration of the project

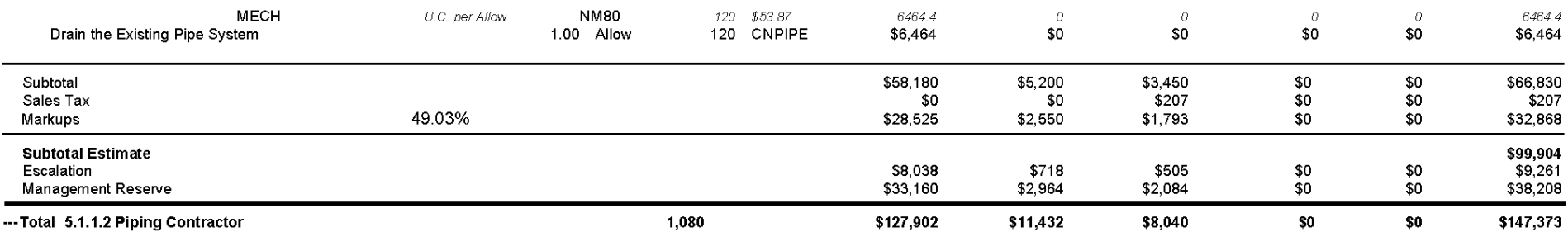

\subsubsection{Electrical Contractor}

Memo: Based on estimators judgment and the duration of the project.

\begin{tabular}{|c|c|c|c|c|c|c|c|c|c|c|c|}
\hline Working Superintendent $20 \%$ & $\begin{array}{l}E L E C T \\
{ }_{6} \text { Admin Duties }\end{array}$ & uc. permo & 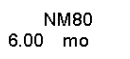 & $\begin{array}{r}32 \\
192\end{array}$ & $\begin{array}{l}\$ 70.54 \\
\text { CNLINEF }\end{array}$ & $\begin{array}{r}2257.28 \\
\$ 13,544\end{array}$ & \$0 & $\$ 0$ & $\begin{array}{l}0 \\
\$ 0\end{array}$ & $\$ 0$ & $\begin{array}{r}225728 \\
\$ 13,544\end{array}$ \\
\hline Electrical Truck Allowance & ELECT & Uc. permo & $\begin{array}{l}\text { NM80 } \\
6.00 \mathrm{mo}\end{array}$ & 0 & & $\$ 0$ & $\begin{array}{r}2100 \\
\$ 12,600\end{array}$ & \$o & \$o & $\$ 0$ & $\begin{array}{r}2,100 \\
\$ 12,600\end{array}$ \\
\hline Electrical Contractor Mob/Dem & ELECT & U. perweeks & $\begin{array}{l}\text { NM80 } \\
2.00 \stackrel{\text { weeks }}{ }\end{array}$ & $\begin{array}{l}20 \\
40\end{array}$ & $\begin{array}{l}\$ 57.25 \\
\text { CNELECGF }\end{array}$ & $\begin{array}{r}1145 \\
\$ 2,290\end{array}$ & $\$ 0$ & $\$ 0$ & $\begin{array}{r}0 \\
\$ 0\end{array}$ & \$o & $\begin{array}{r}1145 \\
\$ 2,290\end{array}$ \\
\hline Electrical Labels & ELECT & UC per Allow & 1.00 NM80 & $\begin{array}{l}40 \\
10\end{array}$ & $\begin{array}{l}\$ 57.25 \\
\text { CNELECGF }\end{array}$ & $\begin{array}{r}2290 \\
\$ 2,290\end{array}$ & $\$ 0$ & $\begin{array}{r}1000 \\
\$ 1,000\end{array}$ & \$o & \$o & $\begin{array}{r}3290 \\
\$ 3,290\end{array}$ \\
\hline Electrical Testing & ELECT & U.C. per Allow & 1.00 Allow & $\begin{array}{l}240 \\
240\end{array}$ & $\begin{array}{l}\$ 57.25 \\
\text { CNELECGF }\end{array}$ & $\begin{array}{r}13740 \\
\$ 13,740\end{array}$ & $\$ 0$ & $\begin{array}{r}150 \\
\$ 150\end{array}$ & $\$ 0$ & $\$ 0$ & $\$ 13,890$ \\
\hline Vendor Data & ELECT & U.C. perallow & $\begin{array}{c}\text { NM80 } \\
1.00 \text { allow }\end{array}$ & $\begin{array}{l}40 \\
40\end{array}$ & $\begin{array}{l}\$ 57.25 \\
\text { CNELECGF }\end{array}$ & $\begin{array}{r}2290 \\
\$ 2,290\end{array}$ & $\$ 0$ & $\$ 0$ & \$o & $\$ 0$ & $\begin{array}{r}2290 \\
\$ 2,290\end{array}$ \\
\hline
\end{tabular}


Project Name: RTC Waste Heat Recovery

Project Location: ATR

Estimate Number: 7850

Code Description

5.1.1.3 Electncal contractor

Memo: Based on estimators judgment and the duration of the project.

\begin{tabular}{|c|c|c|c|c|c|c|c|c|c|}
\hline Sign \& Barriers & U.C perallow & $\begin{array}{c}\text { NM80 } \\
1.00 \text { allow }\end{array}$ & $\begin{array}{ll}40 & \$ 57.25 \\
40 & \text { CNELECGF }\end{array}$ & $\begin{array}{r}22200 \\
\$ 2,290\end{array}$ & $\begin{array}{l}0 \\
\$ 0\end{array}$ & 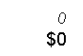 & $\begin{array}{r}0 \\
\$ 0\end{array}$ & 告0 & $\begin{array}{r}2290 \\
\$ 2,290\end{array}$ \\
\hline 5.1.1.3 Electrical Contractor & & & & $\$ 80,118$ & $\$ 27,700$ & $\$ 2,680$ & \$0 & \$o & $\$ 110,498$ \\
\hline
\end{tabular}

Main Supply Demolition (Canal Area $40^{\circ}$ Elevation)

Memo: RSMeans 220102.20 for labor adjustment factors.

$\begin{array}{cclrlrl}\text { MECH } & \text { U.C. per Lnit } & & & 0.455 & \$ 53.87 & 24.532 \\ \text { Overhead Metal Piping Demolition Supply Piping } & & 200.00 & \text { Lnft } & 91 & \text { CNPIPE } & \$ 4,906\end{array}$

Subtotal
Sales Tax 4

Sales Tax

Subtotal Estimate

Escalation
Management Reserve

--Total Maan Supply Demolttion (Canal Area 40" Elevation)

DETAIL ITEM REPORT

Client: C. P. Ischay

Prepared By: A.W.Miller/S. N. Wasley

Estimate Type: Class-5

Qty UOM Hrs Resource Labor Equipment Material Subcontractor Other TOTAL

Main Supply Insulation Demolition (Canal Area 40' Elevation)

Memo: RSMeans 220102.20 for labor adjustment factors. MECH U. per L Piping Insulation Removal (Canal Area) U.C. per Lint 200.00 Lnt

RSMeans 028213.43 for insulation demolition.

BEA

05/11/2012 11:08:15

$\begin{array}{rlllll}\$ 4,906 & \$ 0 & \$ 0 & \$ 0 & \$ 0 & \$ 4,906 \\ \$ 0 & \$ 0 & \$ 0 & \$ 0 & \$ 0 & \$ 0 \\ \$ 2,406 & \$ 0 & \$ 0 & \$ 0 & \$ 0 & \$ 2,406 \\ & & & & & \$ 7,312 \\ \$ 678 & \$ 0 & \$ 0 & \$ 0 & \$ 0 & \$ 678 \\ \$ 2,796 & \$ 0 & \$ 0 & \$ 0 & \$ 0 & \$ 2,796 \\ \$ 10, r 86 & \$ 0 & \$ 0 & \$ 0 & \$ 0 & \$ 10,886\end{array}$
$\$ 312$
$\$ 678$
$\$ 2,796$ 10,186
$\begin{aligned} 0.853 & \$ 53.87 \\ 171 & \text { CNPIPE }\end{aligned}$
45.951
$\$ 9,190$

6.55
$\$ 1,310$

$\$$

$\begin{array}{rrr}\$ 0 & 0 & 52.501 \\ \$ 0 & \$ 0 & \$ 10,500\end{array}$

Cost Estimating 
Project Name: RTC Waste Heat Recovery

Project Location: ATR

Pstimate Number: 7850

Code Description

contractor

Qty UOM Hrs Resource Labor

Equipment

RSMeans 028213.43 for insulation demolition.

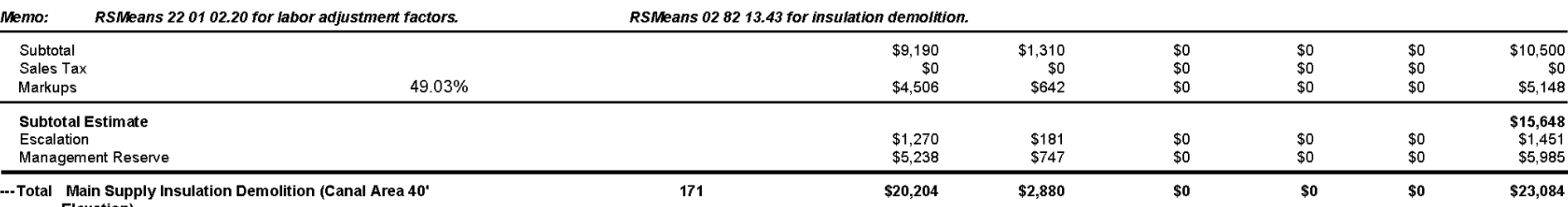

Main Supp
Elevation)

Main Retum Demolition (Canal Area 40. Elevation)

Memo: RSMeans 220102.20 for labor adjustment factors.

$\begin{array}{crlrlrl}\text { MECH } & \text { U.C. per Lnit } & & & 0.455 & \$ 53.87 & 24.532 \\ \text { Overhead Metal Piping Demolition Supply Piping } & & 200.00 & \text { Lnft } & 91 & \text { CN PIPE } & \$ 4,906\end{array}$
(Canal Area)

Subtotal

Sales Tax
Markups

Subtotal Estimate

Manalation

---Total Main Retum Demolition (Canal Area 40' Elevation)

$49.03 \%$

200.00 Lnft

Client:

C.P. Ischay

Estimate Ty. A.W. Miller/S. N. Wasley

stimate Type: Class-5

Other TOTAL

$\begin{array}{rlllll}\$ 4,906 & \$ 0 & \$ 0 & \$ 0 & \$ 0 & \$ 4,906 \\ \$ 0 & \$ 0 & \$ 0 & \$ 0 & \$ 0 & \$ 0 \\ \$ 2,406 & \$ 0 & \$ 0 & \$ 0 & \$ 0 & \$ 2,406 \\ & & & & & \$ 7,312 \\ \$ 678 & \$ 0 & \$ 0 & \$ 0 & \$ 0 & \$ 678 \\ \$ 2,796 & \$ 0 & \$ 0 & \$ 0 & \$ 0 & \$ 2,796 \\ \$ \$ 10,786 & \$ 0 & \$ 0 & \$ 0 & \$ 0 & \$ 10,786\end{array}$

Main Retum Insulation Demolition (Canal Area $40^{\circ}$ Elevation)

Memo: RSMeans 220102.20 for labor adjustment factors. Piping Insulation Removal MECH U.C. per L U.C. per Lnit 200.00 Lnft

RSMeans 028213.43 for insulation demolition.

$\begin{array}{cccc} & 0.953 & \$ 53.67 & \\ & 171 & \text { CNPIPE } & \$ 5.95 \\ \text { Inft } & & \$ 9,190\end{array}$


Project Name: RTC Waste Heat Recovery

Project Location: ATR

Pstimate Number: 7850

Code Description

Contractor

Qty UOM Hrs Resource Labor

Equipment

RSMeans 028213.43 for insulation demolition.

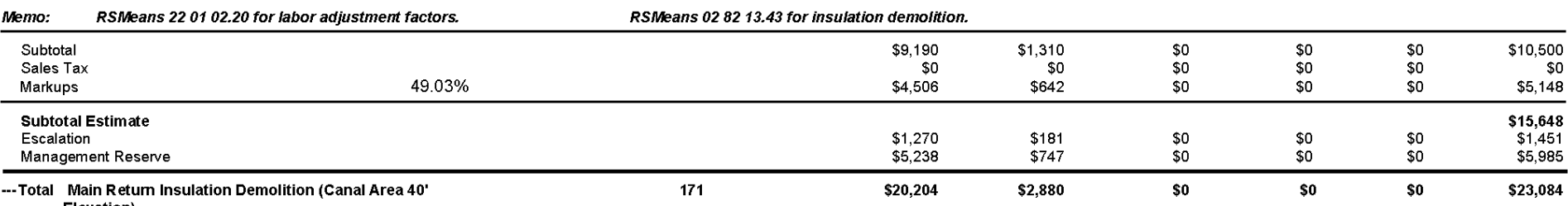

\section{Overhead Working Equipment}

Memo: This is a monthly cost for two snorkel lifts obtained from the Rental Rate Blue Book for Construction Equipment.

\begin{tabular}{|c|c|c|c|c|c|c|c|c|c|}
\hline $\begin{array}{l}\text { MECH } \\
\text { Snorkel Lit (2 Each To Spread The Load Of The } \\
\text { Metal Piping) }\end{array}$ & UC. per Month & 2.00 Month & $\begin{array}{ll}4 & \$ 53.87 \\
8 & \text { CNPIPE }\end{array}$ & $\begin{array}{r}21548 \\
\$ 431\end{array}$ & $\begin{array}{r}5370 \\
\$ 10,740\end{array}$ & $\$ 0$ & $\$$ & $\$ 0$ & $\begin{array}{r}5565.48 \\
\$ 11,171\end{array}$ \\
\hline $\begin{array}{l}\text { Subtotal } \\
\text { Sales Tax } \\
\text { Markups }\end{array}$ & $49.03 \%$ & & & $\begin{array}{r}\$ 431 \\
\$ 0 \\
\$ 211\end{array}$ & $\begin{array}{r}\$ 10,740 \\
\$ 0 \\
\$ 5,266\end{array}$ & $\begin{array}{l}\$ 0 \\
\$ 0 \\
\$ 0\end{array}$ & $\begin{array}{l}\$ 0 \\
\$ 0 \\
\$ 0\end{array}$ & $\begin{array}{l}\$ 0 \\
\$ 0 \\
\$ 0\end{array}$ & $\begin{array}{r}\$ 11,171 \\
\$ 0 \\
\$ 5,477\end{array}$ \\
\hline $\begin{array}{l}\text { Subtotal Estimate } \\
\text { Escalation } \\
\text { Management Reserve }\end{array}$ & & & & $\begin{array}{r}\$ 60 \\
\$ 246\end{array}$ & $\begin{array}{l}\$ 1,484 \\
\$ 6,121\end{array}$ & $\begin{array}{l}\$ 0 \\
\$ 0\end{array}$ & $\begin{array}{l}\$ 0 \\
\$ 0\end{array}$ & 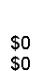 & $\begin{array}{r}\$ 16,648 \\
\$ 1,543 \\
\$ 6,367\end{array}$ \\
\hline Total Overhead Working Equipment & & & & $\$ 947$ & $\$ 23,611$ & $\$ 0$ & \$o & $\$ 0$ & $\$ 24,558$ \\
\hline
\end{tabular}

Main Supply Demolition (Canal Mezzanine Area 40. Elevation)

RSMeans 22010220 for hoor adjustment ractors.

RSMeans 220505.10 for labor to demo existing piping.

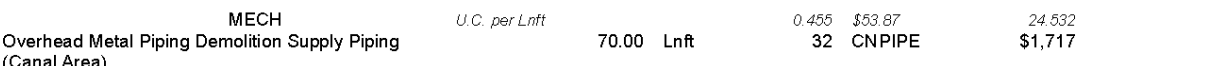

BE 
Project Name: RTC Waste Heat Recovery

Project Location: ATR

Code Descriptit

Contractor

Qty UOM Hrs Resource Labor

Equipment

RSMeans 220505.10 for labor to demo existing piping.

Subtotal
Sales Tax
Markups

Subtotal Estimat

Escalation
Management Reserve

-- -Total Main Supply Demolition (Canal Mezzanine Area 40'
Elevation)

Main Supply Insulation Demolition (Canal Mezzanine Area 40' Elevation

Memo: RSMeans 220102.20 for labor adjustment factors.

RSMeans 028213.43 for insulation demolition.

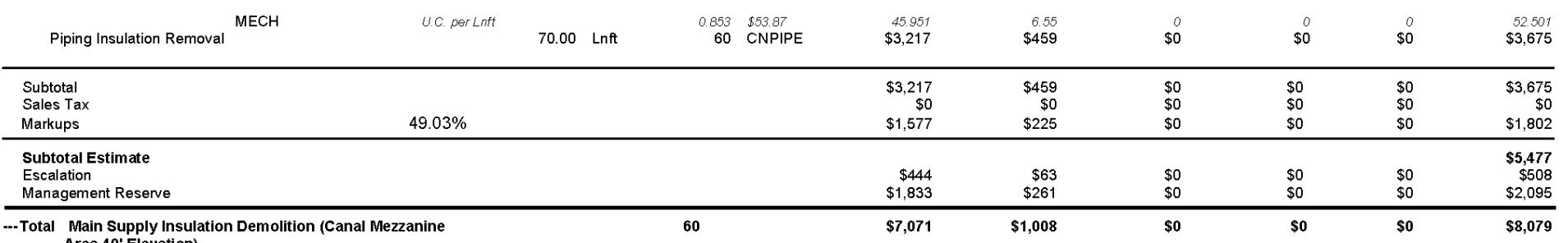

Main Supply Insula
Area 40' Elevation)

Main Retum Demolition (Canal Mezzanine Area 40' Elevation)

Memo: RSMeans 220102.20 for labor adjustment factors.

MECH
Overhead Metal Piping Demolition Supply Piping U. per $L$ int

RSMeans 220505.10 for labor to demo existing piping.

(Canal Area)

BEA

11:08:15

C. P. Ischay

Prepared By: A.W. Miller/S. N. Wasle

mate Type: Class-5

Material Subcontractor Other TOTAL

\begin{tabular}{rllllr}
$\$ 1,717$ & $\$ 0$ & $\$ 0$ & $\$ 0$ & $\$ 0$ & $\$ 1,717$ \\
$\$ 0$ & $\$ 0$ & $\$ 0$ & $\$ 0$ & $\$ 0$ & $\$ 0$ \\
$\$ 842$ & $\$ 0$ & $\$ 0$ & $\$ 0$ & $\$ 0$ & $\$ 842$ \\
& & & & & $\$ 2,559$ \\
$\$ 237$ & $\$ 0$ & $\$ 0$ & $\$ 0$ & $\$ 0$ & $\$ 237$ \\
$\$ 979$ & $\$ 0$ & $\$ 0$ & $\$ 0$ & $\$ 0$ & $\$ 979$ \\
\hline 3,775 & $\$ 0$ & $\$ 0$ & $\$ 0$ & $\$ 0$ & $\$ 3,775$
\end{tabular}

$\begin{array}{lll}0.455 & \$ 53.67 & 24.532\end{array}$

$\$ 0$

$\$ 0$

$\$ 0$

$\$ \begin{array}{ll}0 & 24.532 \\ \$ 0 & \$ 1,717\end{array}$ 
Project Name: RTC Waste Heat Recovery

Project Location: ATR

Pstimate Number: 7850

Code Description $\quad$ Contractor

Maln Retum Demolltion (Canal Mlezzanine Area 40' Elevation)

Memo: RSMeans 22010220 ror moradistmem tactors

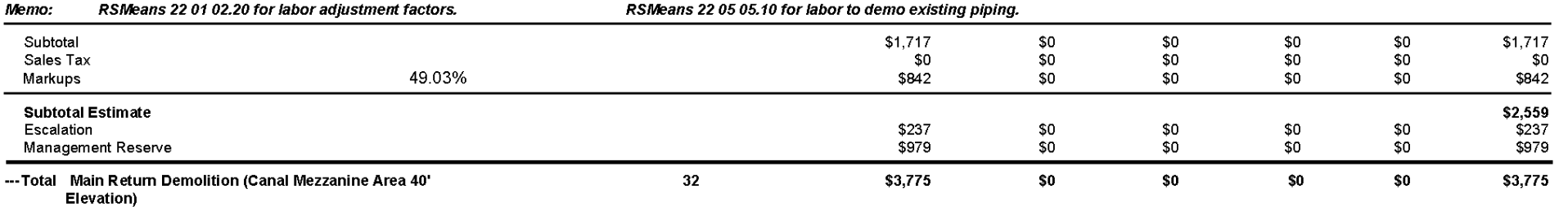

Main Retu
Elevation)

Main Retum Insulation Demolition (Canal Mezzanine Area 40' Elevation

Memo: RSMeans 220102.20 for labor adjustment factors.

RSMeans 028213.43 for insulation demolition.

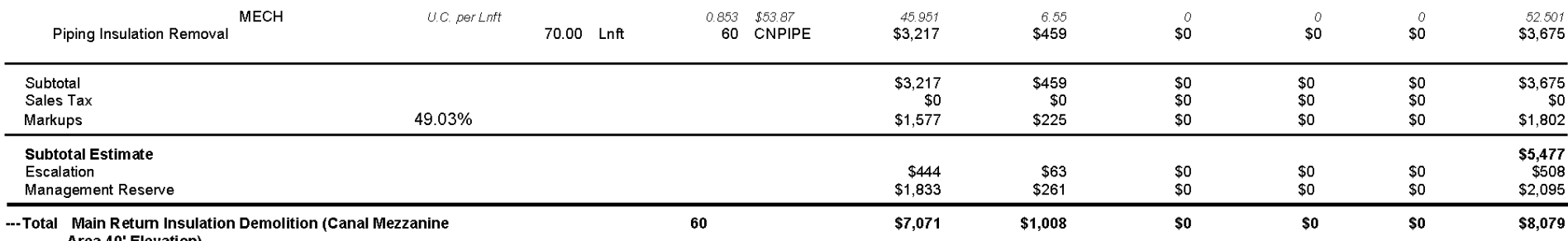

\section{Overhead Workinq Equipment}

Memo: This is a monthly cost for two snorkel lifts obtained from the Rental Rate Blue Book for Construction Equipment.

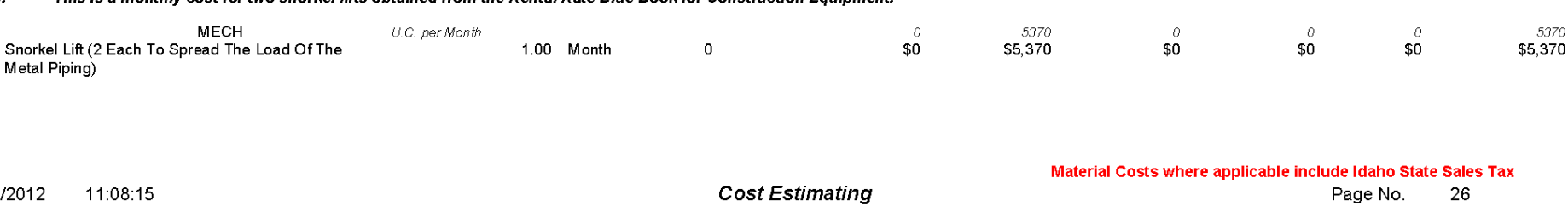

Client: C. P. Ischay

Prepared By: A.W. Miller/S. N. Wasley

timate Type: Class-5

Qty UOM Hrs Resource Labor Equipment Material Subcontractor Other TOTAL

Qty UOM Hrs Resource Labor Equipment Material Subcontractor Other TOTAL

\section{7} 979 
Project Name: RTC Waste Heat Recovery

Project Location: ATR

Pstimate Number: 7850

Code Description contractor

overnead working Equipment

Memo: This is a mombly cost for two snorkel lifts obtained from the Rental Rate Blue Book for Constuction Equphes

\begin{tabular}{|c|c|c|c|c|c|c|c|}
\hline $\begin{array}{l}\text { Subtotal } \\
\text { Sales Tax } \\
\text { Markups }\end{array}$ & $49.03 \%$ & $\begin{array}{l}\$ 0 \\
\$ 0 \\
\$ 0\end{array}$ & $\begin{array}{r}\$ 5,370 \\
\$ 0 \\
\$ 2,633\end{array}$ & $\begin{array}{l}\$ 0 \\
\$ 0 \\
\$ 0\end{array}$ & $\begin{array}{l}\$ 0 \\
\$ 0 \\
\$ 0\end{array}$ & $\begin{array}{l}\$ 0 \\
\$ 0 \\
\$ 0\end{array}$ & $\begin{array}{r}\$ 5,370 \\
\$ 0 \\
\$ 2,633\end{array}$ \\
\hline $\begin{array}{l}\text { Subtotal Estimate } \\
\text { Escalation } \\
\text { Management Reserve }\end{array}$ & & $\begin{array}{l}\$ 0 \\
\$ 0\end{array}$ & $\begin{array}{r}\$ 742 \\
\$ 3,061\end{array}$ & $\begin{array}{l}\$ 0 \\
\$ 0\end{array}$ & $\begin{array}{l}\$ 0 \\
\$ 0\end{array}$ & $\$ 0$ & $\begin{array}{r}\$ 8,003 \\
\$ 742 \\
\$ 3,061\end{array}$ \\
\hline
\end{tabular}

Main Supply Demolition (West Canal Area 30' Elevation)
DETAIL ITEM REPORT

C. P. Ischay

Prepared By: A. W. Miller/S. N. Wasle

Estimate Type: Class-5 
Project Name: RTC Waste Heat Recovery

Project Location: ATR

Code Description

Contractor

Qty UOM Hrs Resource Labor

Equipment

RSMeans 028213.43 for insulation demolition.

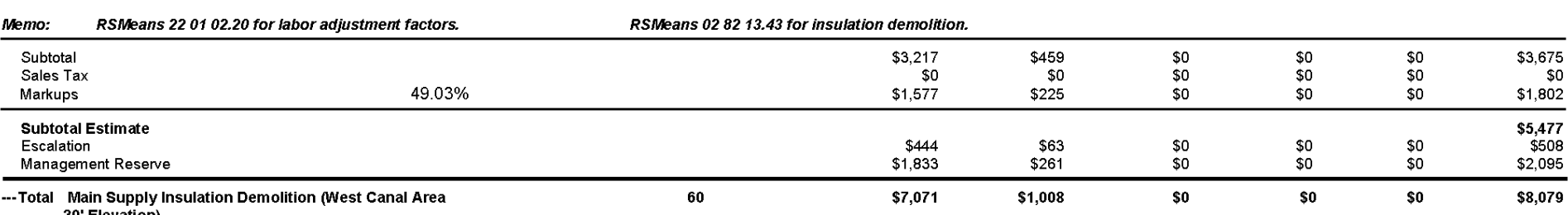

Main Supply In
30' Elevation)

Main Retum Demolition (West Canal Area $30^{\circ}$ Elevation)

Memo: RSMeans 220102.20 for labor adjustment factors.

$\begin{array}{lllllll}\text { MECH } & \text { U.C. per Lnit } & & & 0.455 & 553.87 & 24.532 \\ \begin{array}{l}\text { Overhead Metal Piping Demolition Supply Piping } \\ \text { (Canal Area) }\end{array} & & 70.00 & \text { Lnft } & 32 & \text { CNPIPE } & \$ 1,717\end{array}$
Overhead Meta

Subtotal

Sales Tax
Markups

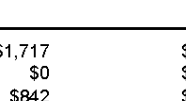

Subtotal Estimat

Escalation

-- Total Main Retum Demolition (West Canal Area 30'
Elevation)

$49.03 \%$

Client: C. P. Ischay

Prepared By: A. W. Miller/S. N. Wasle

Estimate Type: Class-5

Material Subcontractor Other TOTAL

Main Retum Insulation Demolition (West Canal Area 30' Elevation)

Memo: RSMeans 220102.20 for labor adjustment factors.

RSMeans 028213.43 for insulation demolition.

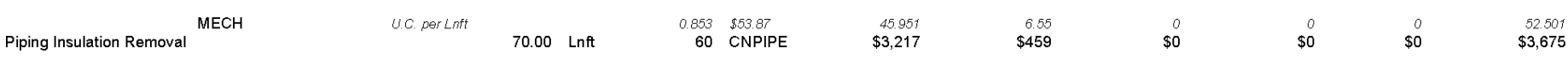

BEA

11:08:15

Cost Estimating

Material costs where applicable include Idaho State Sales Tax

Page No. $\quad 28$ 
Project Name: RTC Waste Heat Recovery

Project Location: ATR

Pstimate Number: 7850

Code Description

Contractor

Qty UOM Hrs Resource Labor

Equipment

RSMeans 028213.43 for insulation demolition.

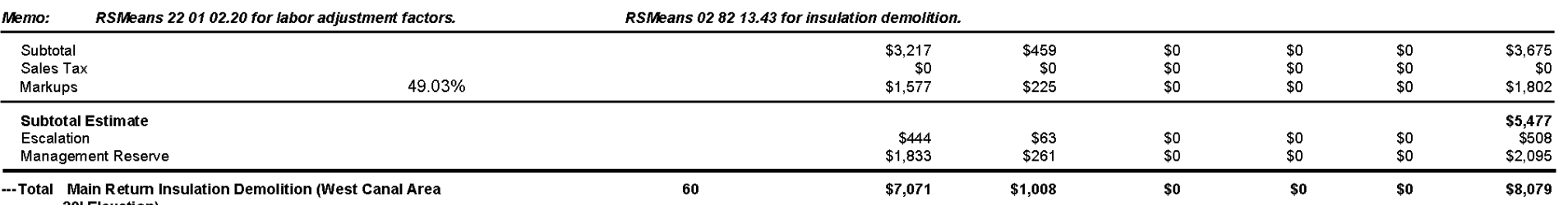

Main Retum In
30' Elevation)

\section{Overhead Working Equipment}

Memo: This is a monthly cost for two snorkel lifts obtained from the Rental Rate Blue Book for Construction Equipment.

\begin{tabular}{|c|c|c|c|c|c|c|c|c|c|}
\hline $\begin{array}{l}\text { MECH } \\
\text { Snorkel Lit (2 Each To Spread The Load Of The } \\
\text { Metal Piping) }\end{array}$ & U.C. perMonth & 1.00 Month & $\begin{array}{l}4 \\
4\end{array}$ & $\$$ & $\begin{array}{r}5370 \\
\$ 5,370\end{array}$ & $\$$ & $\$$ & $\$ 0$ & $\begin{array}{r}5370 \\
\$ 5,370\end{array}$ \\
\hline $\begin{array}{l}\text { Subtotal } \\
\text { Sales Tax } \\
\text { Markups }\end{array}$ & $49.03 \%$ & & & $\begin{array}{l}\$ 0 \\
\$ 0 \\
\$ 0\end{array}$ & $\begin{array}{r}\$ 5,370 \\
\$ 0 \\
\$ 2,633\end{array}$ & $\begin{array}{l}\$ 0 \\
\$ 0 \\
\$ 0\end{array}$ & $\begin{array}{l}\$ 0 \\
\$ 0 \\
\$ 0\end{array}$ & $\begin{array}{l}\$ 0 \\
\$ 0 \\
\$ 0\end{array}$ & $\begin{array}{r}\$ 5,370 \\
\$ 0 \\
\$ 2,633\end{array}$ \\
\hline $\begin{array}{l}\text { Subtotal Estimate } \\
\text { Escalation } \\
\text { Management Reserve }\end{array}$ & & & & $\begin{array}{l}\$ 0 \\
\$ 0\end{array}$ & $\begin{array}{r}\$ 742 \\
\$ 3,061\end{array}$ & $\begin{array}{l}\$ 0 \\
\$ 0\end{array}$ & $\begin{array}{l}\$ 0 \\
\$ 0\end{array}$ & $\begin{array}{l}\$ 0 \\
\$ 0\end{array}$ & $\begin{array}{r}\$ 8,003 \\
\$ 742 \\
\$ 3,061\end{array}$ \\
\hline
\end{tabular}

Main Supply Demolition (West Canal Area 40' Elevation)

Memo: RSMeans 220102.20 for labor adjustment factors

$\mathrm{MECH}$
Vertical Metal Piping Demolition Supply Piping
(Canal Area)

uc. per Lnit

RSMeans 220505.10 for labor to demo existing piping.

30.00 Lnft

$\begin{array}{rl}0.455 & 553.87 \\ 14 & \text { CNPIPE }\end{array}$

24.532
$\$ 736$

$\$ 0$

Client:

Prepared By: A. W. Miller/S. N. Wasle

Estimate Type: Class-5

terial Subcontractor Other TOTAL

DETAIL ITEM REPORT

BEA 
Project Name: RTC Waste Heat Recovery

Project Location: ATR

Code Description

Contractor

Qty UOM Hrs Resource Labor

Equipment

RSMeans 220505.10 for labor to demo existing piping.

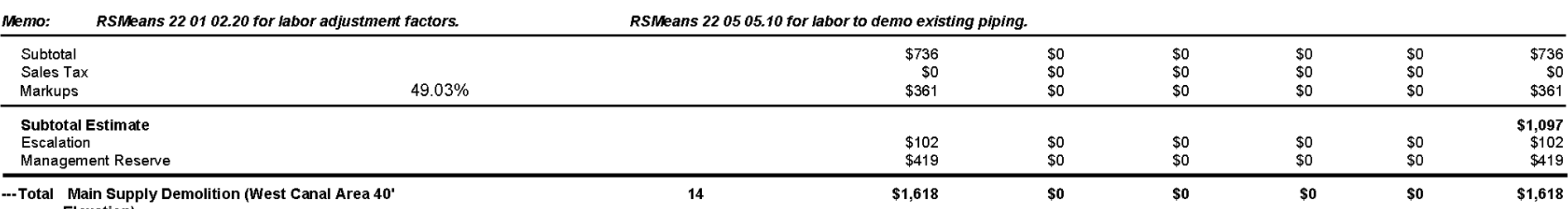

Main Supp
Elevation)

Main Supply Insulation Demolition (West Canal Area 40 Elevation)

Memo: RSMeans 220102.20 for labor adjustment factors.

RSMeans 028213.43 for insulation demolition.

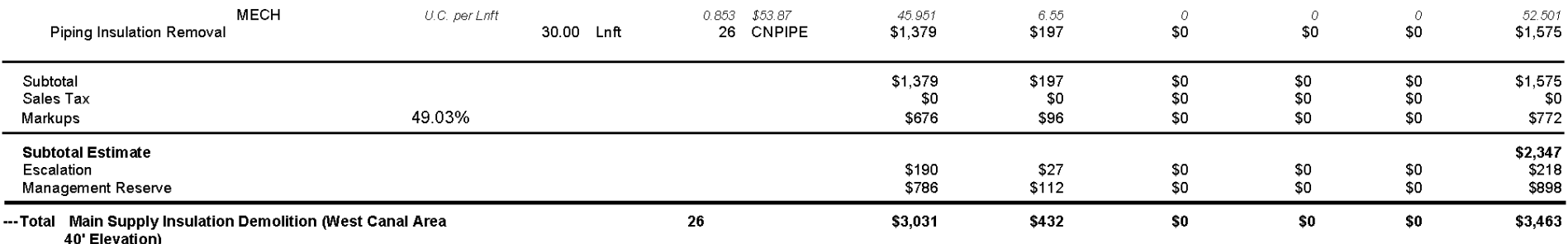

Main Retum Demolltion (West Canal Area 40 Elevation

Memo: RSMeans 220102.20 for labor adjustment factors.

$\mathrm{MECH}$
Vertical Metal Piping Demolition Supply Piping
(Canal Area)

Means 220505.10 for labor to demo existing piping.$$
\text { (Canal Area) }
$$

BEA

05/11/2012 11:08:15
Prepared By: A.W. Miller/S. N. Wasle

Estimate Type: Class-5

Material Subcontractor Other TOTAL $\begin{array}{r}\$ 736 \\ \$ 0 \\ \$ 361 \\ \$ 1097 \\ 102 \\ \hline 19 \\ \hline 18\end{array}$
Client: C.P. Ischay
U.C. per Lnit $30.00 \quad$ Lnft

U.C. per Lnit $30.00 \quad$ Lnft

(2) $\begin{array}{rrr}0.455 & 553.67 & 24.532 \\ 14 & \text { CNPIPE } & \$ 736\end{array}$

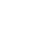

$\begin{array}{rrrrr} & 0 & 0 & 0 & 24532 \\ \$ 0 & \$ 0 & \$ 0 & \$ 0 & \$ 736\end{array}$


Project Name: RTC Waste Heat Recovery

Project Location: ATR

Code Description $\quad$ contractor Wlain Retum Demolltion (west Canal Area $40^{\circ}$ Elevation)

Memo: RSMeans 22010220 for habor adjustment factors.

\begin{tabular}{|c|c|c|c|c|c|c|c|}
\hline $\begin{array}{l}\text { Memo: } \\
\text { Subtotal } \\
\text { Sales Ta }\end{array}$ & RSMeans 220102.20 for labor adjustment factors. & \multicolumn{5}{|c|}{ RSMeans 220505.10 for labor to demo existing piping. } & \multirow{2}{*}{$\begin{array}{r}\$ 736 \\
\$ 0 \\
\$ 361\end{array}$} \\
\hline Markups & & & & & & & \\
\hline $\begin{array}{l}\text { Subtotal } \\
\text { Escalatio } \\
\text { Manager }\end{array}$ & $\begin{array}{l}\text { Estimate } \\
\text { n } \\
\text { nent Reserve }\end{array}$ & $\begin{array}{l}\$ 102 \\
\$ 419\end{array}$ & $\begin{array}{l}\$ 0 \\
\$ 0\end{array}$ & $\begin{array}{l}\$ 0 \\
\$ 0\end{array}$ & $\begin{array}{l}\$ 0 \\
\$ 0\end{array}$ & $\begin{array}{l}\$ 0 \\
\$ 0\end{array}$ & $\begin{array}{r}\$ 1,097 \\
\$ 102 \\
\$ 419\end{array}$ \\
\hline- -Total $\underset{\substack{\mathrm{Ele} \\
\mathrm{Ele}}}{\mathrm{M}}$ & $\begin{array}{l}\text { ain Retum Demolition (West Canal Area 40' } \\
\text { evation) }\end{array}$ & $\$ 1,618$ & \$o & so & so & so & $\$ 1,618$ \\
\hline
\end{tabular}

Main Retum Insulation Demolition (West Canal Area 40' Elevation)

Memo: RSMeans 220102.20 for labor adjustment factors.

RSMeans 028213.43 for insulation demolition.

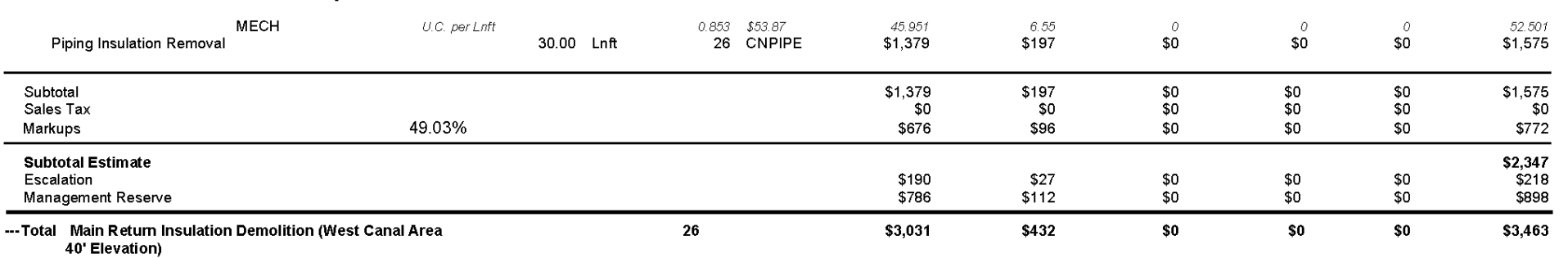

\section{Overhead Working Equipment}

Memo: This is a monthly cost for two snorkel lifts obtained from the Rental Rate Blue Book for Construction Equipment

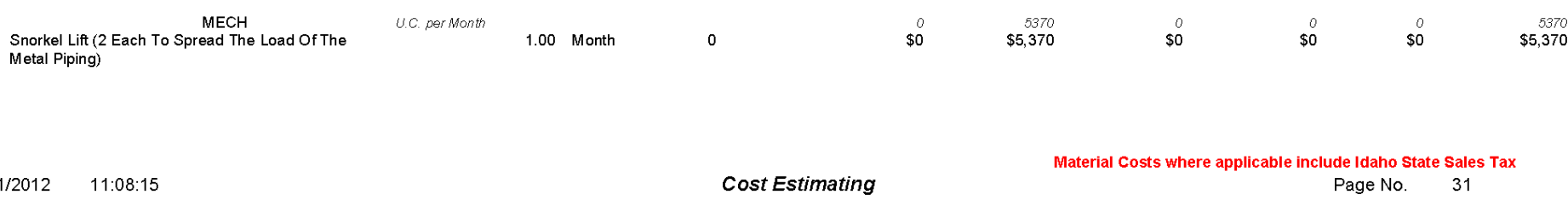

Client: C. Ischay

Prepared By: A. W. Miller/S. N. Wasley
Estimate Type: Class-5

Qty UOM Hrs Resource Labor Equipment Material Subcontractor Other TOTAL

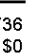
102 $\$ 419$ 
Project Name: RTC Waste Heat Recovery

Project Location: ATR

Pstimate Number: 7850

Code Description Contractor

overnead working Equipment

Memo: This is a mombly cost for two snorkel lifts obtained trom the Rental Rate Blue Book for Constuction Equphes

\begin{tabular}{|c|c|c|c|c|c|c|c|}
\hline $\begin{array}{l}\text { Subtotal } \\
\text { Sales Tax } \\
\text { Markups }\end{array}$ & $49.03 \%$ & $\begin{array}{l}\$ 0 \\
\$ 0 \\
\$ 0\end{array}$ & $\begin{array}{r}\$ 5,370 \\
\$ 0 \\
\$ 2,633\end{array}$ & $\begin{array}{l}\$ 0 \\
\$ 0 \\
\$ 0\end{array}$ & $\begin{array}{l}\$ 0 \\
\$ 0 \\
\$ 0\end{array}$ & $\begin{array}{l}\$ 0 \\
\$ 0 \\
\$ 0\end{array}$ & $\begin{array}{r}\$ 5,370 \\
\$ 0 \\
\$ 2,633\end{array}$ \\
\hline $\begin{array}{l}\text { Subtotal Estimate } \\
\text { Escalation } \\
\text { Management Reserve }\end{array}$ & & $\begin{array}{l}\$ 0 \\
\$ 0\end{array}$ & $\begin{array}{r}\$ 742 \\
\$ 3,061\end{array}$ & $\begin{array}{l}\$ 0 \\
\$ 0\end{array}$ & $\begin{array}{l}\$ 0 \\
\$ 0\end{array}$ & $\$ 0$ & $\begin{array}{r}\$ 8,003 \\
\$ 742 \\
\$ 3,061\end{array}$ \\
\hline
\end{tabular}

Main Supply Demolition Laydown Area West Wall 20' Elevation

Memo: RSMeans 220102.20 for labor adjustment factors.

RSMeans 220505.10 for labor to demo existing piping

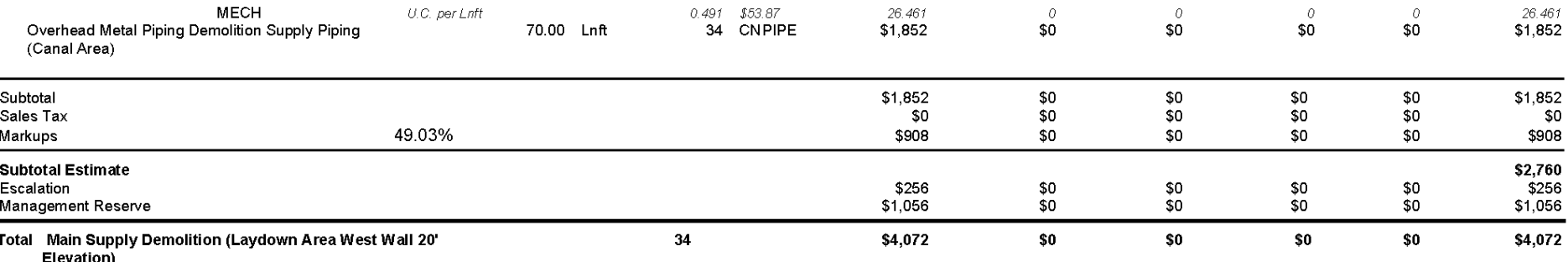

Main Supply Insulation Demolition (Laydown Area West Wall 20' Elevation)

Memo: RSMeans 220102.20 for labor adjustment factors.

RSMeans 028213.43 for insulation demolition.

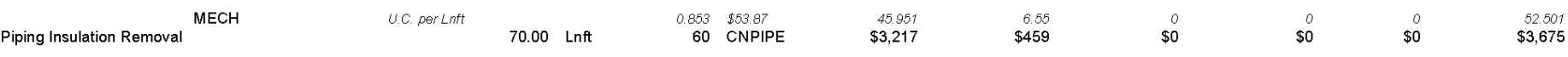


Project Name: RTC Waste Heat Recovery

Project Location: ATR

Estimate Number: 7850

Code Description

Contractor

Qty UOM Hrs Resource Labor

Equipment

RSMeans 028213.43 for insulation demolition.

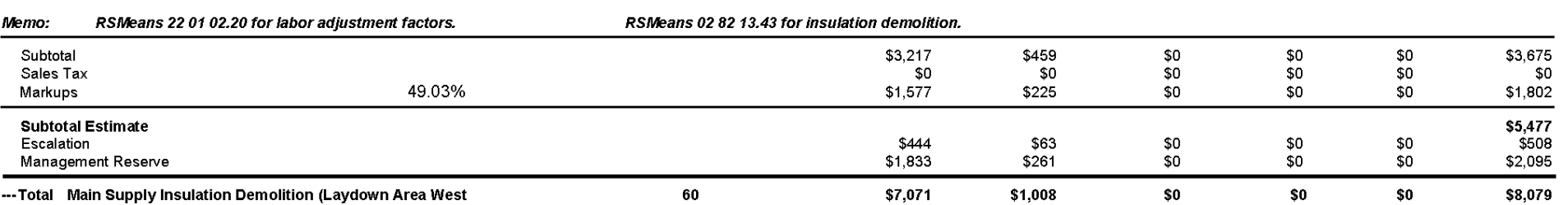

Main Supply Insul
Wall $20^{\prime}$ Elevation)

Main Return Demolition (Laydown Area West Wall 20' Elevation

Memo: RSMeans 220102.20 for labor adjustment factors.

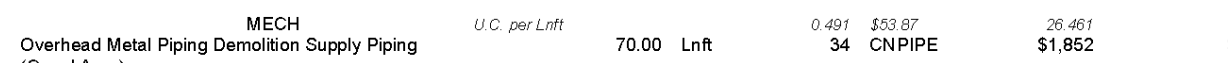
(Canal Area)

Subtotal

Sales Tax
Markups

(N)

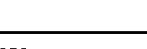

Subtotal Estimate

Escalation
Management Reserve

-Total Main Retum

Main Retur
Elevation)

\section{Main Return Insulation Demolition (Laydown Area West Wall 20' Elevation)}

Memo: RSMeans 220102.20 for labor adjustment factors.

RSMeans 028213.43 for insulation demolition.
Piping Insulation Removal MECH
u.c. per Lnit
$\begin{array}{rl}0.853 & 553.87 \\ 60 & \text { CNPIPE }\end{array}$

70.00 Lnft

45.557
$\$ 3,217$

6.55
$\$ 459$

$\$ 0$

$\$$

$\$ 0$

52.501
$\$ 3,675$
Prepared By: A.W. Miller/S. N. Wasley

Subcontractor Other TOTAL timate Type: Class-5
BEA

05/11/2012 11:08:15
Cost Estimating 
Project Name: RTC Waste Heat Recovery

Project Location: ATR

Estimate Number: 7850

Code Description

Contractor

Qty UOM Hrs Resource Labor

Equipment

RSMeans 028213.43 for insulation demolition.

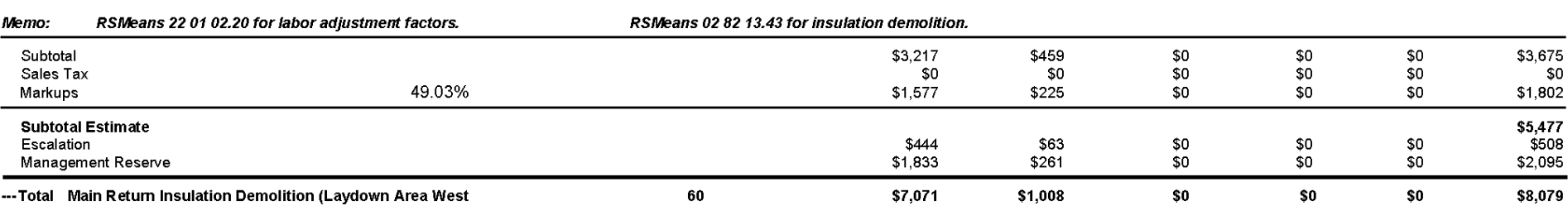

Wail $20^{\prime}$ Elevation)

\section{Overhead Working Equipment}

Memo: This is a monthly cost for two snorkel lifts obtained from the Rental Rate Blue Book for Construction Equipment.

\begin{tabular}{|c|c|c|c|c|c|c|c|c|c|}
\hline $\begin{array}{l}\text { MECH } \\
\text { Snorkel Lit ( } 2 \text { Each To Spread The Load Of The } \\
\text { Metal Piping) }\end{array}$ & U.C. per Month & 1.00 Month & 0 & $\$ 0$ & $\begin{array}{r}5370 \\
\$ 5,370\end{array}$ & $\$ 0$ & $\$$ & $\stackrel{0}{\$ 0}$ & $\begin{array}{r}5370 \\
\$ 5,370\end{array}$ \\
\hline $\begin{array}{l}\text { Subtotal } \\
\text { Sales Tax } \\
\text { Markups }\end{array}$ & $49.03 \%$ & & & $\begin{array}{l}\$ 0 \\
\$ 0 \\
\$ 0\end{array}$ & $\begin{array}{r}\$ 5,370 \\
\$ 0 \\
\$ 2,633\end{array}$ & $\begin{array}{l}\$ 0 \\
\$ 0 \\
\$ 0\end{array}$ & $\begin{array}{l}\$ 0 \\
\$ 0 \\
\$ 0\end{array}$ & $\begin{array}{l}\$ 0 \\
\$ 0 \\
\$ 0\end{array}$ & $\begin{array}{r}\$ 5,370 \\
\$ 0 \\
\$ 2,633\end{array}$ \\
\hline $\begin{array}{l}\text { Subtotal Estimate } \\
\text { Escalation } \\
\text { Management Reserve }\end{array}$ & & & & $\begin{array}{l}\$ 0 \\
\$ 0\end{array}$ & $\begin{array}{r}\$ 742 \\
\$ 3,061\end{array}$ & $\begin{array}{l}\$ 0 \\
\$ 0\end{array}$ & $\begin{array}{l}\$ 0 \\
\$ 0\end{array}$ & $\begin{array}{l}\$ 0 \\
\$ 0\end{array}$ & $\begin{array}{r}\$ 8,003 \\
\$ 742 \\
\$ 3,061\end{array}$ \\
\hline Overhead Wo & & & & $\$ 0$ & $\$ 11,805$ & $\$ 0$ & $\$ 0$ & $\$ 0$ & $\$ 11,805$ \\
\hline
\end{tabular}

Main Supply Demolition (Laydown Area North Wall 20' Elevation

Memo: RSMeans 220102.20 for labor adjustment factors.

RSMeans 220505.10 for labor to demo existing piping.

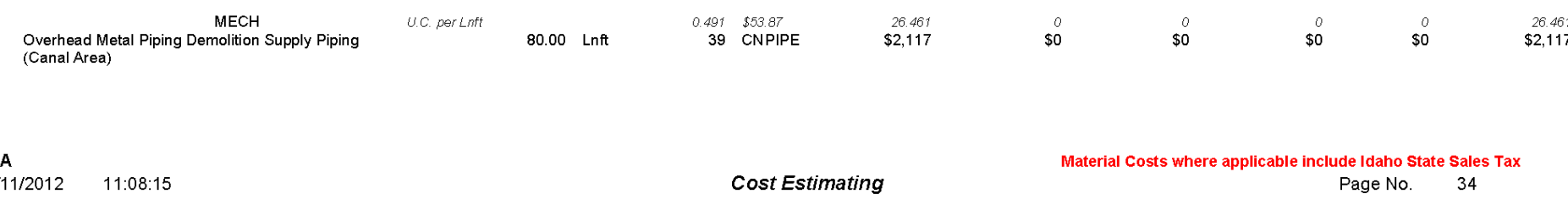

Client: C.P.Ischay

Prepared By: A.W.Miller/S. N. Wasley

Estimate Type: Class-5

Material Subcontractor Other TOTAL 477 ,079

\section{DETAIL ITEM REPORT}


Project Name: RTC Waste Heat Recovery

Project Location: ATR

Code Descriptit

Contractor

Qty UOM Hrs Resource Labor

Equipment

RSMeans 220505.10 for labor to demo existing piping.

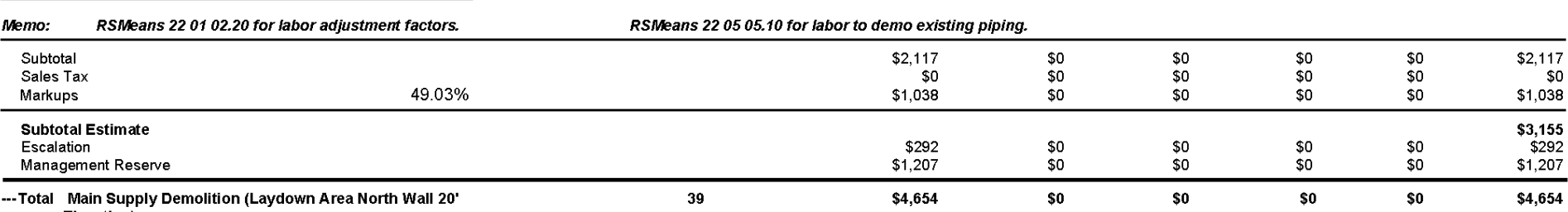

Main Supp
Elevation)

Main Supply Insulation Demolition (Laydown Area North Wall $20^{\circ}$ Elevation)

Memo: RSMeans 220102.20 for labor adjustment factors

RSMeans 028213.43 for insulation demolition.

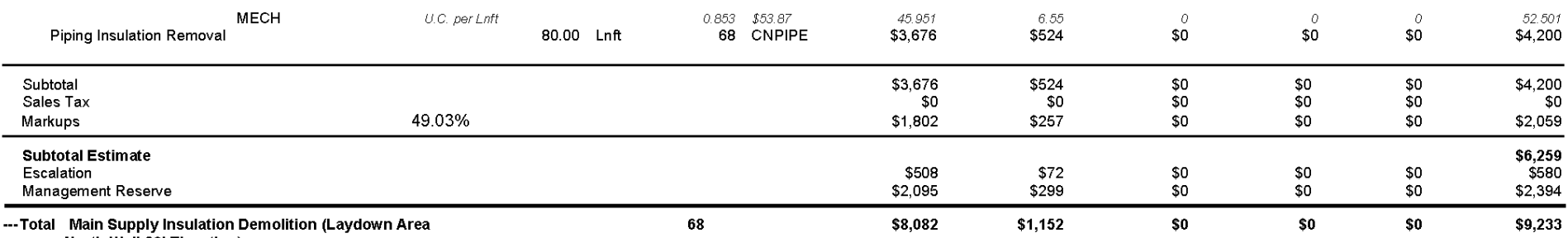

Main Supply Insulation

Main Retum Demolition (Lavdown Area North Wall 20 Elevation)

Memo: RSMeans 220102.20 for labor adjustment factors.

MECH U.o. perL

Overhead Metal Piping Demolition Supply Piping

4.C. per Lint $\quad 80.00$ Lnt

RSMeans 220505.10 for labor to demo existing piping.

5/11/2012 11:08:15
Client: C. P. Ischay

Prepared By: A.W. Miller/S. N. Wasle

Estimate Type: Class-5

Material Subcontractor Other TOTAL

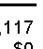
038 5292

$\begin{array}{lll}0.491 & \$ 53.67 & 26,461 \\ 39 & \text { CNPIPE } & \$ 2,117\end{array}$

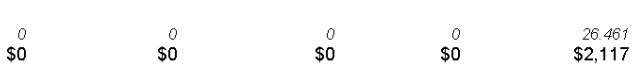


Project Name: RTC Waste Heat Recovery

Project Location: ATR

Code Description

Contractor

Qty UOM Hrs $\underline{\text { Resource Labor }}$

Equipment

RSMeans 220505.10 for labor to demo existing piping.

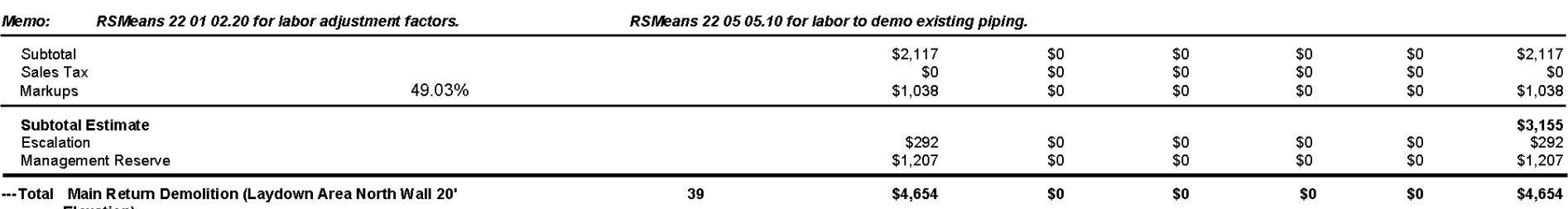

Elevation)

Main Retum Insulation Demolition (Lavdown Area North Wall 20' Elevation)

Memo: RSMeans 220102.20 for labor adjustment factors

RSMeans 028213.43 for insulation demolition

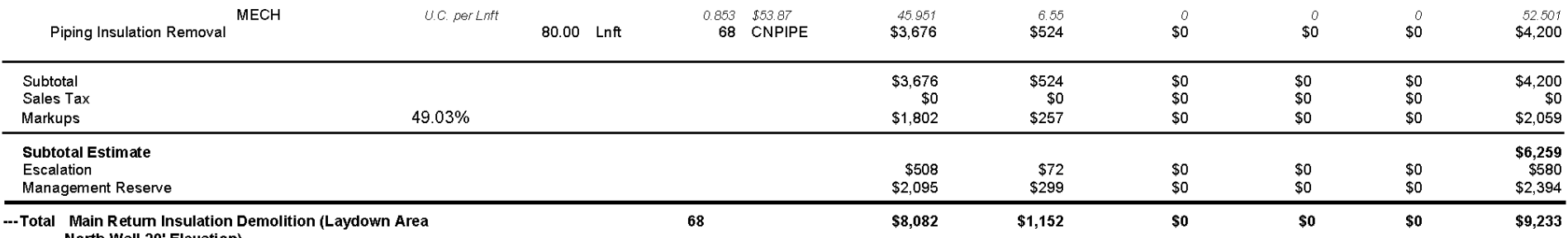

North Wall 20' Elevation

\section{Overhead Working Equipment}

Memo: This is a monthly cost for two snorkel lifts obtained from the Rental Rate Blue Book for Construction Equipment.

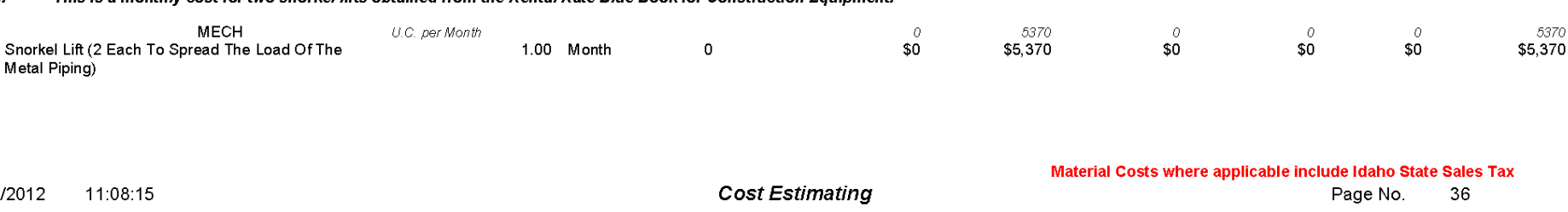

Client: C. P. Ischay

Prepared By: A. W. Miller/S. N. Wasley

Material Subcontractor Other TOTAL

\section{$\begin{array}{r}117 \\ \$ 0 \\ \$ 38 \\ \hline 155 \\ \$ 292 \\ \hline 107 \\ \hline 5654\end{array}$}


Project Name: RTC Waste Heat Recovery

Project Location: ATR

Pstimate Number: 7850

Code Description Contractor

overnead working Equipment

Memo: This is a mombly cost for two snorkel lifts obtained from the Rental Rate Blue Book for Constuction Equphes

\begin{tabular}{|c|c|c|c|c|c|c|c|}
\hline $\begin{array}{l}\text { Subtotal } \\
\text { Sales Tax } \\
\text { Markups }\end{array}$ & $49.03 \%$ & $\begin{array}{l}\$ 0 \\
\$ 0 \\
\$ 0\end{array}$ & $\begin{array}{r}\$ 5,370 \\
\$ 0 \\
\$ 2,633\end{array}$ & $\begin{array}{l}\$ 0 \\
\$ 0 \\
\$ 0\end{array}$ & $\begin{array}{l}\$ 0 \\
\$ 0 \\
\$ 0\end{array}$ & $\begin{array}{l}\$ 0 \\
\$ 0 \\
\$ 0\end{array}$ & $\begin{array}{r}\$ 5,370 \\
\$ 0 \\
\$ 2,633\end{array}$ \\
\hline $\begin{array}{l}\text { Subtotal Estimate } \\
\text { Escalation } \\
\text { Management Reserve }\end{array}$ & & $\begin{array}{l}\$ 0 \\
\$ 0\end{array}$ & $\begin{array}{r}\$ 742 \\
\$ 3,061\end{array}$ & $\begin{array}{l}\$ 0 \\
\$ 0\end{array}$ & $\begin{array}{l}\$ 0 \\
\$ 0\end{array}$ & $\$ 0$ & $\begin{array}{r}\$ 8,003 \\
\$ 742 \\
\$ 3,061\end{array}$ \\
\hline
\end{tabular}

Main Supply Demolition (Southwest Area 40' Elevation

Memo: RSMeans 220102.20 for labor adjustment factors

RSMeans 220505.10 for labor to demo existing piping.

\begin{tabular}{|c|c|c|c|c|c|c|c|c|c|}
\hline $\begin{array}{l}\text { MECH } \\
\text { Overhead Metal Piping Demolition Supply Piping } \\
\text { (Canal Area) }\end{array}$ & uc per Lnit & 140.00 Lnft & $\begin{aligned} 0.491 & \$ 53.87 \\
69 & \text { CNPIPE }\end{aligned}$ & $\begin{array}{r}26.467 \\
\$ 3,705\end{array}$ & $\$ 0$ & $\$ 0$ & $\$ 0$ & $\$ 0$ & $\begin{array}{r}26.461 \\
\$ 3,705\end{array}$ \\
\hline $\begin{array}{l}\text { Subtotal } \\
\text { Sales Tax } \\
\text { Markups }\end{array}$ & $49.03 \%$ & & & $\begin{array}{r}\$ 3,705 \\
\$ 0 \\
\$ 1,816\end{array}$ & $\begin{array}{l}\$ 0 \\
\$ 0 \\
\$ 0\end{array}$ & $\begin{array}{l}\$ 0 \\
\$ 0 \\
\$ 0\end{array}$ & $\begin{array}{l}\$ 0 \\
\$ 0 \\
\$ 0\end{array}$ & $\begin{array}{l}\$ 0 \\
\$ 0 \\
\$ 0\end{array}$ & $\begin{array}{r}\$ 3,705 \\
\$ 0 \\
\$ 1,816\end{array}$ \\
\hline $\begin{array}{l}\text { Subtotal Estimate } \\
\text { Escalation } \\
\text { Management Reserve }\end{array}$ & & & & $\begin{array}{r}\$ 512 \\
\$ 2,111\end{array}$ & $\begin{array}{l}\$ 0 \\
\$ 0\end{array}$ & $\begin{array}{l}\$ 0 \\
\$ 0\end{array}$ & $\begin{array}{l}\$ 0 \\
\$ 0\end{array}$ & $\begin{array}{l}\$ 0 \\
\$ 0\end{array}$ & $\begin{array}{r}\$ 5,521 \\
\$ 512 \\
\$ 2,111\end{array}$ \\
\hline -Total Main Supply Demolitit & & & 69 & $\$ 8,144$ & $\$ 0$ & $\$ 0$ & $\$ 0$ & \$0 & $\$ 8,144$ \\
\hline
\end{tabular}

Main Supply Insulation Demolition (Southwest Area 40' Elevation)

Memo: RSMeans 220102.20 for labor adjustment factors.

RSMeans 028213.43 for insulation demolition.

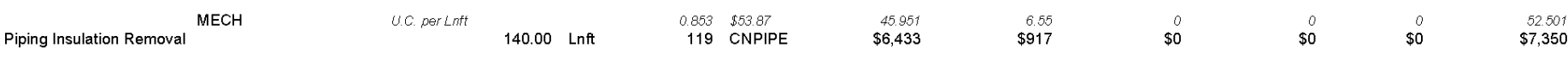


Project Name: RTC Waste Heat Recovery

Project Location: ATR

Code Description

Contractor

Qty UOM Hrs Resource Labor

Equipment

Maln Supply insulation Demolltion (Southwest Area $40^{\circ}$ Elevation

Memo:

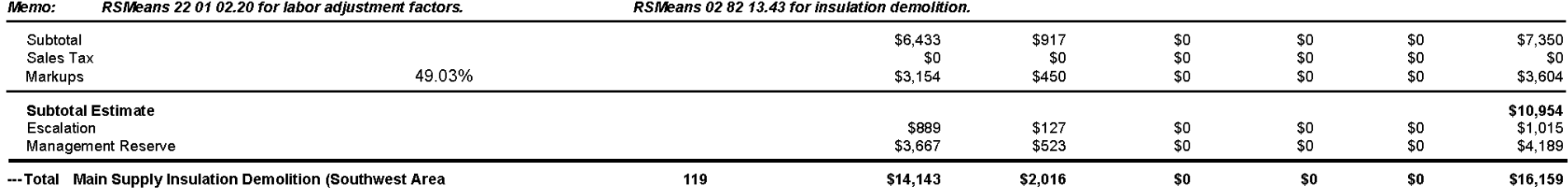

Main Return Demolition (Southwest Area 40' Elevation)

Memo: RSMeans 220102.20 for labor adjustment factors.

$\begin{array}{crlrlrl}\text { MECH } & \text { U.C. per Lnit } & & & 0.491 & \$ 53.87 & 26.461 \\ \text { Overhead Metal Piping Demolition Supply Piping } & & 140.00 & \text { Lnft } & 69 & \text { CN PIPE } & \$ 3,705\end{array}$
(Canal Area)

Subtotal

Sales Tax
Markups

tangs

Client:

Prepared By: A. W. Miller/S. N. Wasley
Estimate Type: Class-5

subcontractor Other TOTAL

$49.03 \%$

$\$ 3,705$
$\$ 0$
$\$ 1,816$$\quad \$ \$ 0$

Management Reserve

Total Main Retum Demolition (Southwest Area $40^{\circ}$
Elevation)

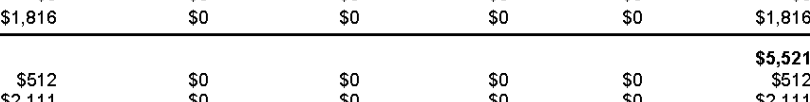

Main Return Insulation Demolition (Southwest Area 40' Elevation)

Memo: RSMeans 220102.20 for labor adjustment factors.

RSMeans 028213.43 for insulation demolition.

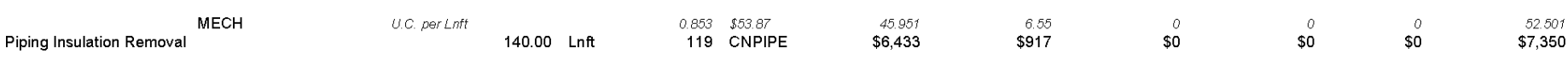

11:08:15

Cost Estimating

Material costs where applicable include Idaho State Sales Tax

Page No. 38 
Project Name: RTC Waste Heat Recovery

Project Location: ATR

Code Description

Contractor

Qty UOM Hrs Resource Labor

Equipment

RSMeans 028213.43 for insulation demolition.

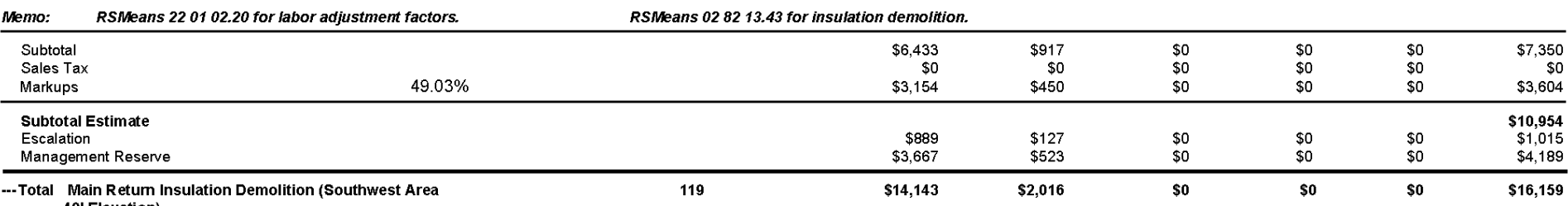

\section{Overhead Working Equipment}

Memo: This is a monthly cost for two snorkel lifts obtained from the Rental Rate Blue Book for Construction Equipment.

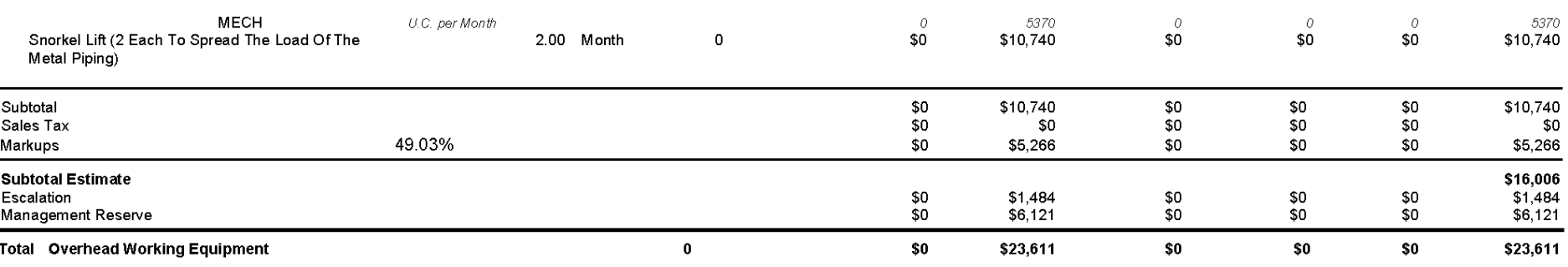

Main Supply Demolition (Diesel Generator Room Area North Wall 20' Elevation)

Memo: RSMeans 220102.20 for labor adjustment factors.

RSMeans 220505.10 for labor to demo existing piping.

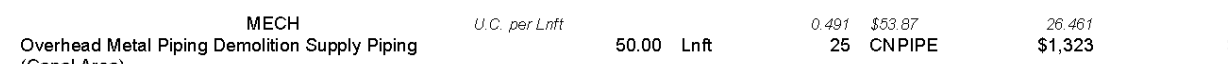
Overhead Meta f

25 CNPIPE

\$o

Client:

Prepared By: A. W. Miller/S. N. Wasle

Estimate Type: Class-5

Material Subcontractor Other TOTAL

$\begin{array}{ll}\text { BEA } & \\ \text { 05/11/2012 } & 11: 08: 15\end{array}$

Cost Estimating

Material costs where applicable include Idaho State Sales Tax

Page No. $\quad 39$ 
Project Name: RTC Waste Heat Recovery

Project Location: ATR

Estimate Number: 7850

Code Description

Contractor

Qty UOM Hrs Resource Labor

Equipment

\section{(ang}

Memo: RSMeans 220102.20 for labor adjustment factors. RSMeans 220505.10 for labor to demo existing piping.

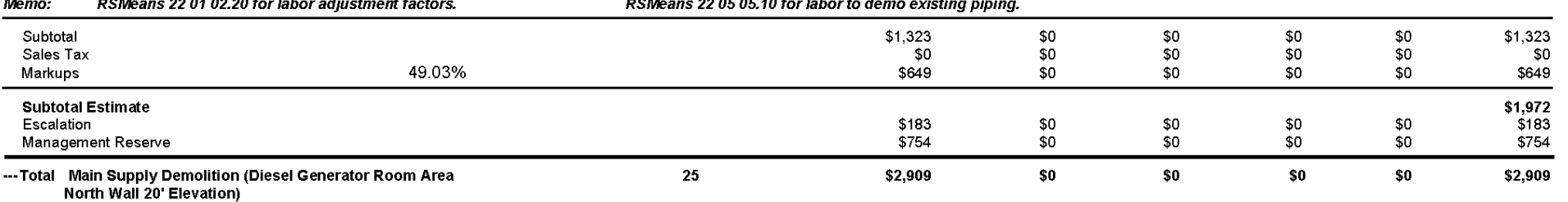

Main Supply Insulation Demolition (Diesel Generator Room Area North Wall 20' Elevation)

Memo: RSMeans 220102.20 for labor adjustment factors.

RSMeans 028213.43 for insulation demolition.

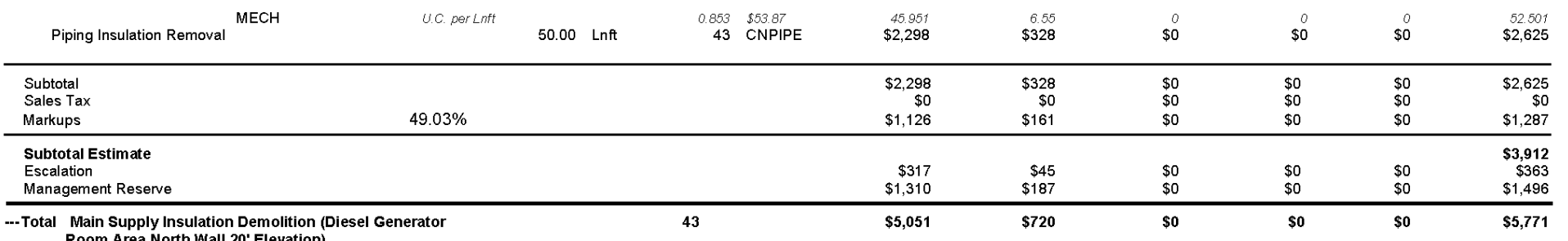

Room Area North Wall 20' Elevation)

Main Retum Demollition (Diesel Generator Room Area North Wall 20 Elevation)

Memo: RSMeans 220102.20 for labor adjustment factors.

RSMeans 220505.10 for labor to demo existing piping.

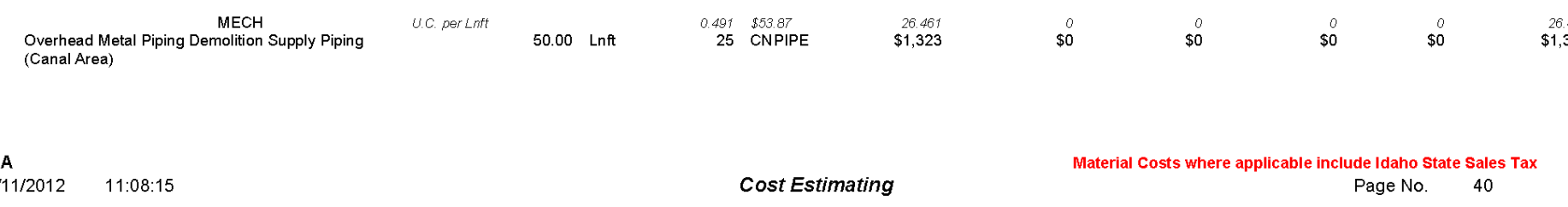

Client: C. P. Ischay

Prepared By: A.W. Miller/S. N. Wasley

Estimate Type: Class-5

Material Subcontractor Other TOTAL 54

Total Main Supply Demolition (Diesel Generator Room Area 
Project Name: RTC Waste Heat Recovery

Project Location: ATR

Estimate Number:7850

Code Description

Contractor

Qty UOM Hrs Resource Labor

Equipment

valin Return Demolltion (Dlesel Generator Room Area North wall 20" Elevation)

Memo: RSMeans 220102.20 for labor adjustment factors. $\quad$ RSMeans 220505.10 for labor to demo existing piping.

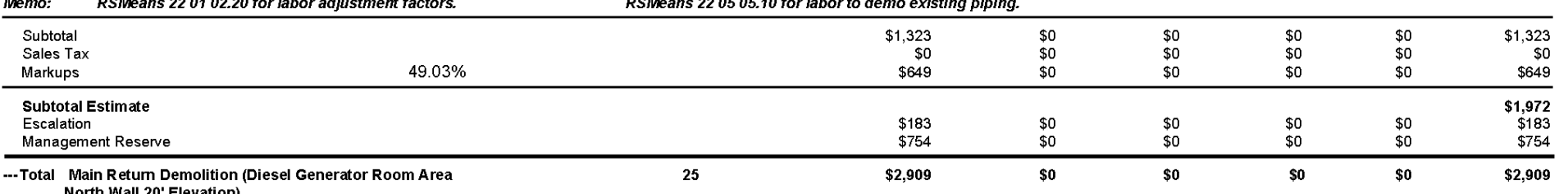

Main Retum Demolition
North Wall 20' Elevation)

Main Return Insulation Demolition (Diesel Generator Room Area West Wall 20 Elevation)

Memo: RSMeans 220102.20 for labor adjustment factors. RSMeans 028213.43 for insulation demolition.

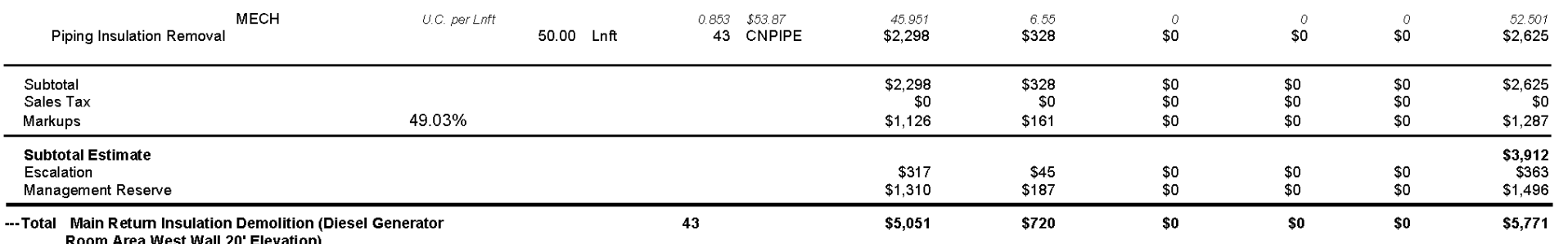

Room Area West Wall 20' Elevation

\section{Overhead Working Equipment}

Memo: This is a monthly cost for two snorkel lifts obtained from the Rental Rate Blue Book for Construction Equipment.

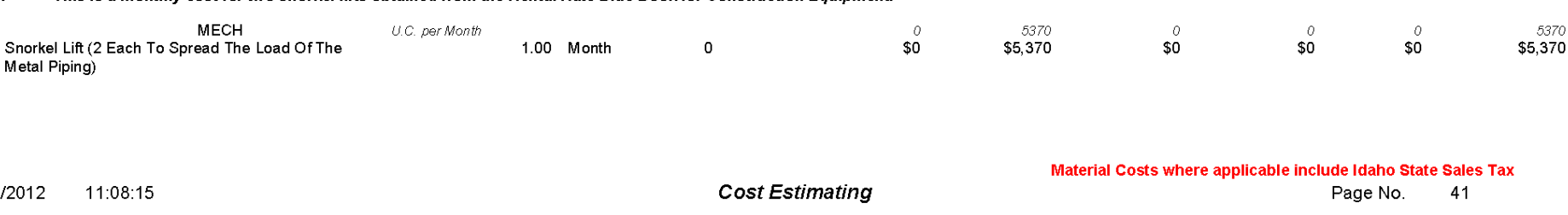

Client: C. Ischay

Prepared By: A.W. Miller/S. N. Wasley

timate Type: Class-5

Material Subcontractor Other TOTAL

\section{754}

$\$ 754$ 
Project Name: RTC Waste Heat Recovery

Project Location: ATR

Code Description $\quad$ contractor

overnead working Equlpment

Memo: This is a monthly cost for two snorkel lifts obtained from the Rental Rate Blue Book for Construction Equipment.

Subtotal

Markups

Markups

Estimate

Management Reserve

--Total Overhead Working Equipment

5.1.21.9 Demo Existing Coils

MECH
Demo Heating Coil HVS-1 (size $\left.10^{\prime} \times 12^{\prime}\right)$

U.C. per SF

$120.00 \mathrm{SF}$

$\begin{array}{rl}0.15 & 553.87 \\ 18 & \text { CNPIPE }\end{array}$

$\underset{2\left(\text { size } 10^{\prime} \times 12^{\prime}\right)}{\mathrm{MECH}}$

$$
\text { U.C. per SF }
$$

$120.00 \mathrm{SF}$

$\begin{array}{ll}0.15 & \$ 53.87 \\ 18 & \text { CNPIPE }\end{array}$

U.C. per SF

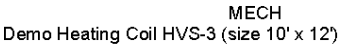

$\mathrm{MECH}$
Demo Heating Coil HVS-4 (size $\left.10^{\prime} \times 12\right)$

U. . per SF

$120.00 \mathrm{SF}$

$\begin{aligned} 0.15 & \$ 53.87 \\ 18 & \text { CNPIPE }\end{aligned}$

$\begin{aligned} 0.15 & \$ 53.67 \\ 18 & \text { CNPIPE }\end{aligned}$

$120.00 \mathrm{SF}$
DETAIL ITEM REPORT

Client: C.P.Ischay

Prepared By: A. W. Miller/S. N. Wasley
Estimate Type: Class-5

Equipment Material Subcontractor Other TOTAL

$\begin{array}{lllll} & & & & \\ \$ 5,370 & \$ 0 & \$ 0 & \$ 0 & \$ 5,370\end{array}$

$\begin{array}{rrrrrr}\$ 0 & \$ 5,370 & \$ 0 & \$ 0 & \$ 0 & \$ 5,370 \\ \$ 0 & \$ 0 & \$ 0 & \$ 0 & \$ 0 & \$ 0 \\ \$ 0 & \$ 2,633 & \$ 0 & \$ 0 & \$ 0 & \$ 2,030\end{array}$

\begin{tabular}{rrrrrr}
$\$ 0$ & & & & $\$ 742$ \\
$\$ 0$ & $\$ 0$ & $\$ 0$ & $\$ 0$ & $\$ 7,003$ \\
$\$ 3,061$ & $\$ 0$ & $\$ 0$ & $\$ 0$ & $\$ 3,061$ \\
\hline 50 & $\$ 1,805$ & $\$ 0$ & $\$ 0$ & $\$ 0$ & $\$ 11,805$
\end{tabular}

$\begin{array}{rrrrrr}\$ 0 & \$ 3,061 & \$ 0 & \$ 0 & \$ 0 & \$ 3,061 \\ \$ \$ 0 & \$ 11,805 & \$ 0 & \$ 0 & \$ 0 & \$ 11,805\end{array}$

Material costs where applicable include Idaho State Sales Tax Page No. 
Project Name: RTC Waste Heat Recovery

Project Location: ATR

Code Description

5.1.21.9 Demo ExIsInn Colls

(1)

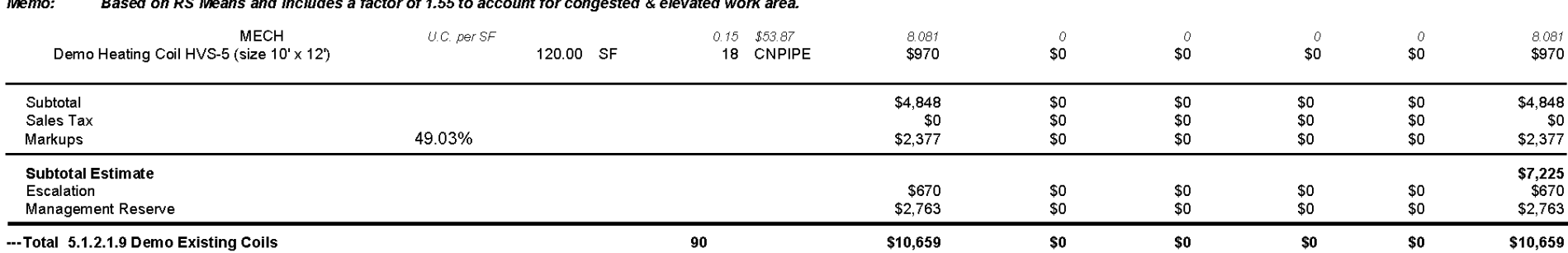

Main Supply Installation (Canal Area 40' Elevation)

$$
\begin{gathered}
\text { MECH } \\
\text { Overhead Metal Piping 8", Installation Supply }
\end{gathered}
$$

200.00 Lnft

$\begin{array}{ll}1.27 & \$ 53.87 \\ 254 & \text { CNPIPE }\end{array}$

68.415
$\$ 13,683$

Client: C.P.Ischay

Prepared By: A. W. Miller/S. N. Wasley
Estimate Type: Class-5

Piping (Canal Area)
mo: RSMeans 2211 13.44 Pipe Steel This has been factored 55\% for the congested area

MECH
8" - Piping Flange Fiting Installatio
Memo: RSMeans 221113.47

$$
\text { UC. per } E a
$$

$3.00 \mathrm{Ea}$

$\begin{array}{ll}4.8 & 553.87 \\ 14 & \text { CNPIPE }\end{array}$

258.577
$\$ 776$

$\begin{aligned} 9 & \$ 53.87 \\ 18 & \text { CNPIPE }\end{aligned}$

484.83
$\$ 970$

ME" - 90 Degree Elbow Pipe Fitting Installation
Memo: RSMeans 221113.47

UC. perEa

2.00 Ea

$4538 t$

8" - 45 Degree Elbow Pipe FECH
Fitting Installation

U.c. perEa

$4.00 \quad \mathrm{Ea}$

$\begin{array}{ll}9 & \$ 55.87 \\ 36 & \text { CNPIPE }\end{array}$

484.83
$\$ 1,939$

MECH
8" - Tee Straight Pipe Fiting Installation
Memo: RSMeans 221113.47

U.C. per Ea

$4.00 \mathrm{Ea}$

$\begin{array}{ll}1344 & 55387 \\ 54 & \mathrm{CNPPF}\end{array}$

724013
$\$ 2896$

Dauipment

Material Subcontractor

Other

TOTAL
5/11/2012 11:08:1 
Project Name: RTC Waste Heat Recovery

Project Location: ATR

Pstimate Number: 7850

\section{Code Description Contractor}

Mlain Suppiy Installation (Canal Area 40" Elevation)

MECH

Gasket and Bolt Set
Memo: RSMeans 221113.47
DETAIL ITEM REPORT

Client: C.P.Ischay

Prepared By: A.W.Miller/S. N. Wasley

Estimate Type: Class-5

Qty UOM Hrs Resource Labor Equipment Material Subcontractor Other TOTAL

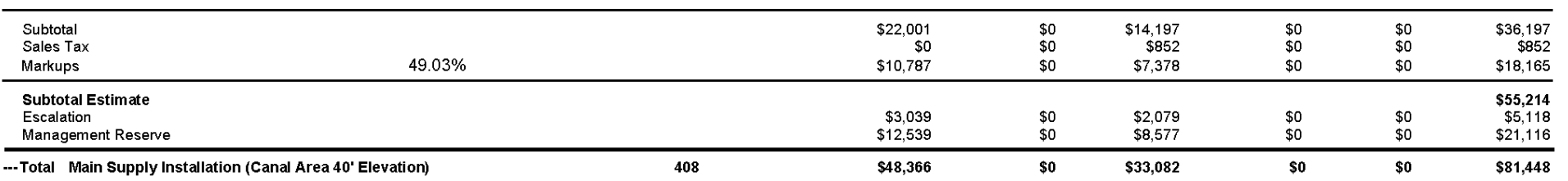

Main Retum Installation (Canal Area 40 Elevation)

$$
\begin{aligned}
& \mathrm{MECH} \\
& \text { Overhead Metal Piping 8", Installation Supply }
\end{aligned}
$$

UC. per Linit 200.00 Lnft

$\begin{array}{ll}127 & 55387 \\ 254 & \mathrm{CNPIPE}\end{array}$

68445
$\$ 13,683$

so

$\$ \begin{array}{r}55 \\ \$ 11,000\end{array}$

so

$\$ 0$

$\underset{\$ 23,683}{12345}$

Memo: RSMeans 221113.44 Pipe Steel This has been factored 55\% for the congested area

8" - Piping Flange Fitting Installation
Memo: RSMeans 221113.47
8" - 90 Degree Elbow Pipe Fitting Installation
Memo: RSMeans 221113.47
8" - 45 Degree Elbow Pipe Fitting Installation
Memo: RSMeans 22 1113.47
8" - Tee Straight Pipe Fiting Installation
Memo: RSMeans 22 1113.47

$$
\text { UC. perEa }
$$

$3.00 \mathrm{Ea}$

$\begin{array}{ll}4.8 & 553.87 \\ 14 & \text { CNPIPE }\end{array}$

258577
$\$ 776$

U.C. per $E a$

2.00 Ea

$\begin{array}{ll}9 & 55387 \\ 18 & \mathrm{CNPPEE}\end{array}$

48483
5970

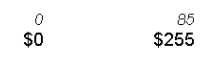

$\$ 0$

so $\quad \begin{aligned} 200 \\ 5400\end{aligned}$

U.c. perEa

$4.00 \mathrm{Ea}$

$\begin{array}{rl}9 & 553.87 \\ 36 & \text { CNPIPE }\end{array}$

484.83
$\$ 1,939$

U.C. per Ea

8" - Tee Straight Pipe Fitting Installation
Memo: RSMeans 221113.47

$4.00 \quad \mathrm{Ea}$

$\begin{array}{ll}1344 & 55387 \\ 54 & \mathrm{CNPPE}\end{array}$

724013
$\$ 2,896$

So $\begin{aligned} 165 \\ 5660\end{aligned}$

$\begin{array}{rr}30 & 355 \\ \$ 0 & \$ 1,420\end{array}$

so

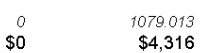

11:08:15
Cost Estimating
Material costs where applicable include Idaho State Sales Tax Page No. $\quad 4$ 
Project Name: RTC Waste Heat Recovery

Project Location: ATR

stimate Number: 7850

\section{Code Description Contractor}

Mvaln Return Installation (CCanal Area 40' Elevation)

MECH

Gasket and Bolt Set
Memo: RSMeans 221113.47

DETAIL ITEM REPORT

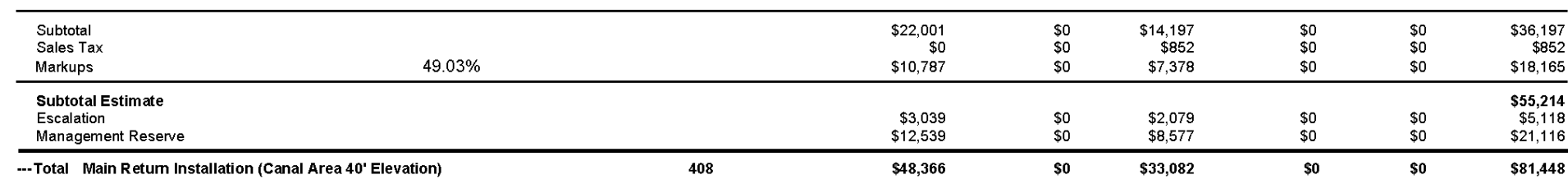

Main Supply Insulation Installation (Canal Area $40^{\prime}$ Elevation)

Memo: RSMeans 220719.30

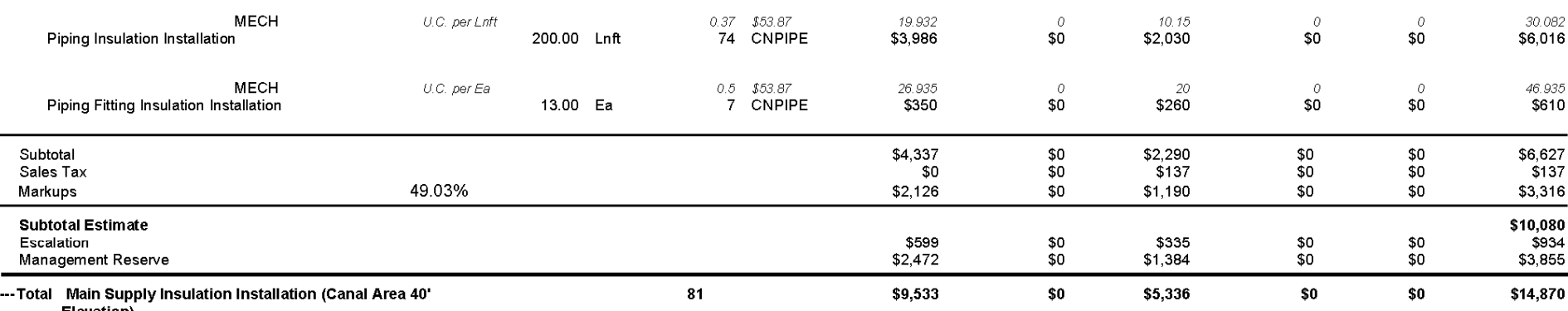

BEA

5/11/2012 11:08:15
Qty UOM Hrs Resource Labor Equipment Material Subcontractor

Other TOTAL

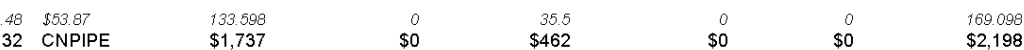
$\$ 852$ $\$ 5,118$ $\$ 1,44$
Material Costs where applicable include Idaho State Sales Tax Page No. $\quad 4$ 
Project Name: RTC Waste Heat Recovery

Project Location: ATR

Estimate Number: 7850

\section{Code Description Contractor}

MMain Retum insulation installation (Canal Area 40. Elevation)

Memo: RSMeans 220719.30

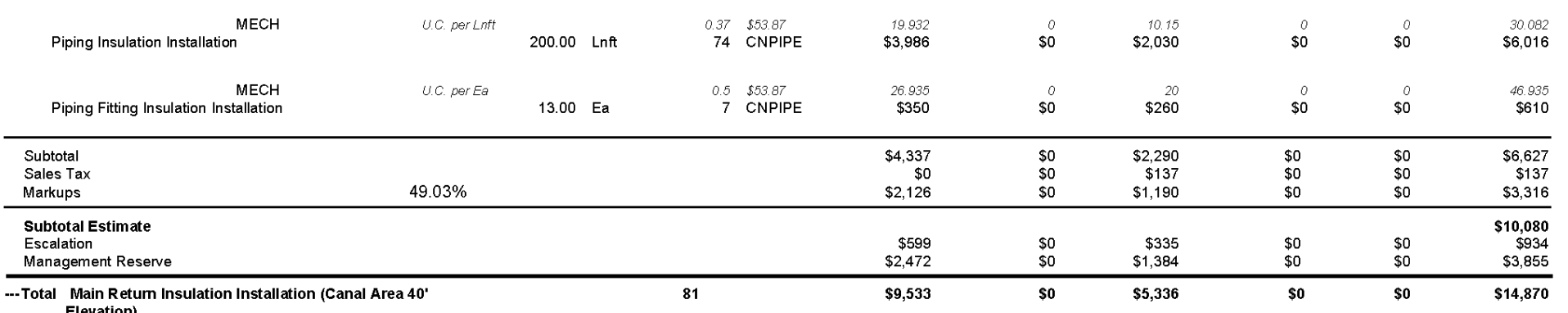

Overhead Working Equipment

Memo: This is a monthly cost for two snorkel Iffs obtained from the Rental Rate Blue Book for Construction Equipment.

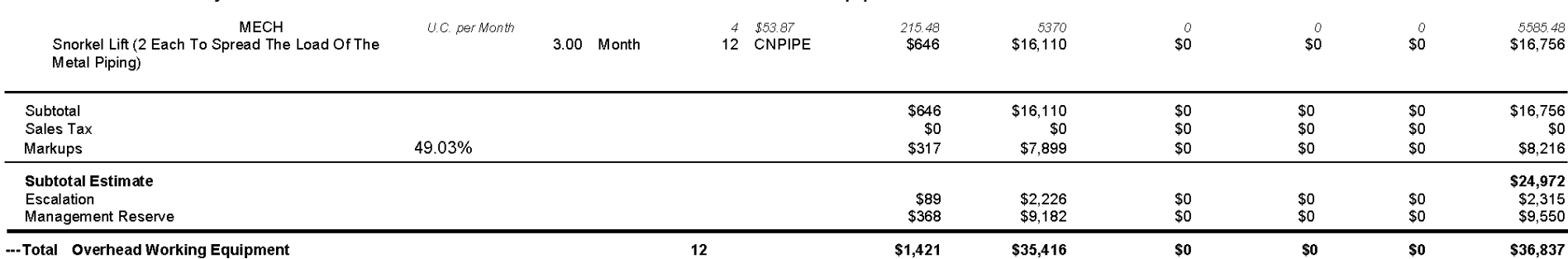


Project Name: RTC Waste Heat Recovery

Project Location: ATR

Estimate Number:7850

\section{Code Description Contractor}

\section{Maln Suppiy Installation (Canal Area 40" Elevation)}

MECH
Overhead Metal Piping Installation Supply Piping

作

Piping Flange Fitting 4", Installation
Memo: RSMeans 2211 13.47

4" - 90 Degree Elbow Pipe Fitting Installation
Memo: RSMeans 221113.47

U.C. per Ea

3.00 Ea

U.C. per Ea

2.00

8 CNPIP

$\begin{array}{rl}4.5 & 553.87 \\ 9 & \text { CNPIPE }\end{array}$

553.87
CNPIPE

C. P. Ischay

Prepared By: A. W. Miller/S. N. Wasley

Estimate Type: Class-5

4" - 45 Degree Elbow Pipe FECHing Installation

U.C. perEa

$4.00 \mathrm{Ea}$

$\begin{array}{ll}4.5 & 553.87 \\ 18 & \text { CNPIPE }\end{array}$

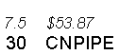

MECH
4" - Tee Straight Pipe Fiting Installation

U.C. per Ea

$4.00 \mathrm{Ea}$

$\begin{aligned} 2.49 & \$ 53.67 \\ 32 & \text { CNPIPE }\end{aligned}$

13.00

Gasket and Bolt Set

\begin{tabular}{|c|c|}
\hline $\begin{array}{l}\text { Subtotal } \\
\text { Sales Tax } \\
\text { Markups }\end{array}$ & $49.03 \%$ \\
\hline $\begin{array}{l}\text { Subtotal Estimate } \\
\text { Escalation } \\
\text { Management Reserve }\end{array}$ & \\
\hline
\end{tabular}

$\$ 7,716$
$\$ 0$
$\$ 3,783$

$\$ 1,066$

$\$ 16,964$ 
Project Name: RTC Waste Heat Recovery

Project Location: ATR

Estimate Number:7850

\section{Code Description Contractor}

MNain Retum Installation (Canal Area 40. Elevation)

\section{DETAIL ITEM REPORT}

lient: C.P.Ischay

Prepared By: A. W. Miller/S. N. Wasley

Estimate Type: Class-5

Qty UOM Hrs Resource Labor Equipment Material Subcontractor Other TOTAL

(a)

U.C. perLnit 70.00 Lnft

$70.00 \operatorname{Lnft}$

Memo. RSMeans 221113.44 Pipe Steel This has been factored $55 \%$ for the congested area

MECH
Piping Flange Fitting 4", Installation

U.C. per Ea

$3.00 \mathrm{Ea}$

$46 \quad$ CNPIPE

35.554
$\$ 2,489$

$\$ 0$

3.5
$\$ 2,450$

35
2,450

$\$ 0$

$\$ 4.539$

RSMeans 221113.47

4" - 90 Degree Elbow Pipe MECH Fiting Installation

U.C. perEa

2.00

$\begin{aligned} 2.6 & \$ 53.87 \\ 8 & \text { CNPIPE }\end{aligned}$

$\$ 40.063$
$\$ 420$

$\begin{array}{rr}0 & 33 \\ \$ 0 & \$ 99\end{array}$

$\$$

$\begin{array}{ll}4.5 & \$ 53.87 \\ 9 & \text { CNPIPE }\end{array}$

242.415
$\$ 485$

48

48
$\$ 96$

$\begin{array}{rrr}0 & 0 & 290.415\end{array}$

\section{U.C. perEa}

$4.00 \mathrm{Ea}$

$\begin{array}{ll}4.5 & \$ 53.87 \\ 18 & \text { CNPIPE }\end{array}$

242.415
$\$ 970$

$\begin{array}{rr}0 & 49.5 \\ \$ 0 & \$ 198\end{array}$

49.5
$\$ 198$

so

MECH
Memo: - Tee Straight Pipe Fitting Installation

U.c. perEa

$4.00 \mathrm{Ea}$

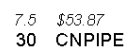

$\underset{\substack{4002028 \\ \text { S1.616 }}}{5.16}$

$\$ 0$

$\$ 472$

$\$ 33.596$
$\$ 1,737$

35.5
$\$ 462$

Gasket and Bolt Set
Memo: RSMeans 221113.47

U.C. per Ea

13.00

J2 CNPIP

$\$ 7,716$
$\$ 0$
$\$ 3,783$

$\$ 7,716$
$\$ 0$
$\$ 3,783$

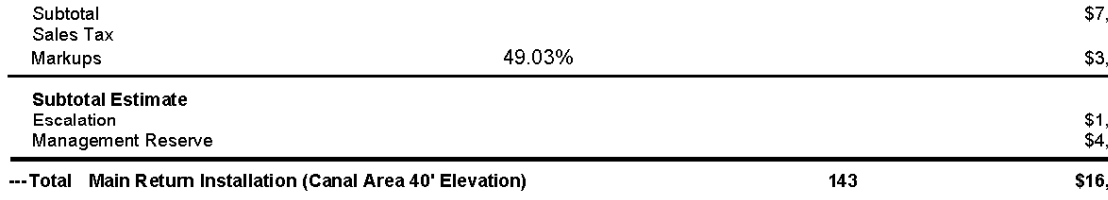

$\$ 1,066$
$\$ 4,398$

$\$ 16,964$

\begin{tabular}{lrrrr}
$\$ 0$ & $\$ 3,777$ & $\$ 0$ & $\$ 0$ & $\$ 11,493$ \\
$\$ 0$ & $\$ 227$ & $\$ 0$ & $\$ 0$ & $\$ 227$ \\
$\$ 0$ & $\$ 1,963$ & $\$ 0$ & $\$ 0$ & $\$ 5,746$ \\
\hline & & & & $\$ 17,465$ \\
$\$ 0$ & $\$ 553$ & $\$ 0$ & $\$ 0$ & $\$ 1,196$ \\
$\$ 0$ & $\$ 2,282$ & $\$ 0$ & $\$ 0$ & $\$ 6,680$ \\
\hline$\$ 0$ & $\$ 8,800$ & $\$ 0$ & $\$ 0$ & $\$ 25,764$
\end{tabular}

BEA

15/11/2012 $\quad 11: 08: 15$
Cost Estimating
Material costs where applicable include Idaho State Sales Tax Page No. 4 
Project Name: RTC Waste Heat Recovery

Project Location: ATR

Estimate Number: 7850

\section{Code Description contractor}

Maain Suppiv Insulation Installation (Canal Area 40' Elevation)

Memo: RSMeans 220719.30

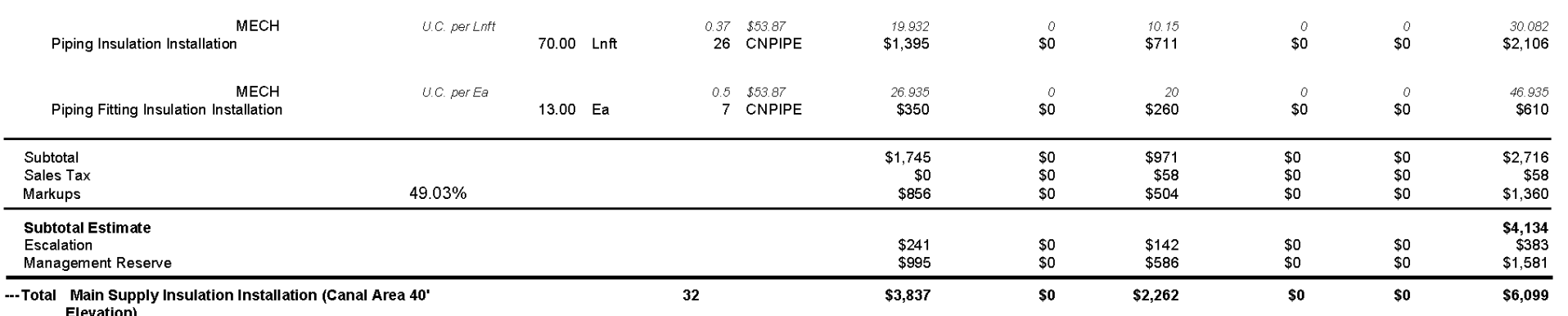

Main Retum Insulation Installation (Canal Area 40' Elevation)

Memo: RSMeans 220719.30

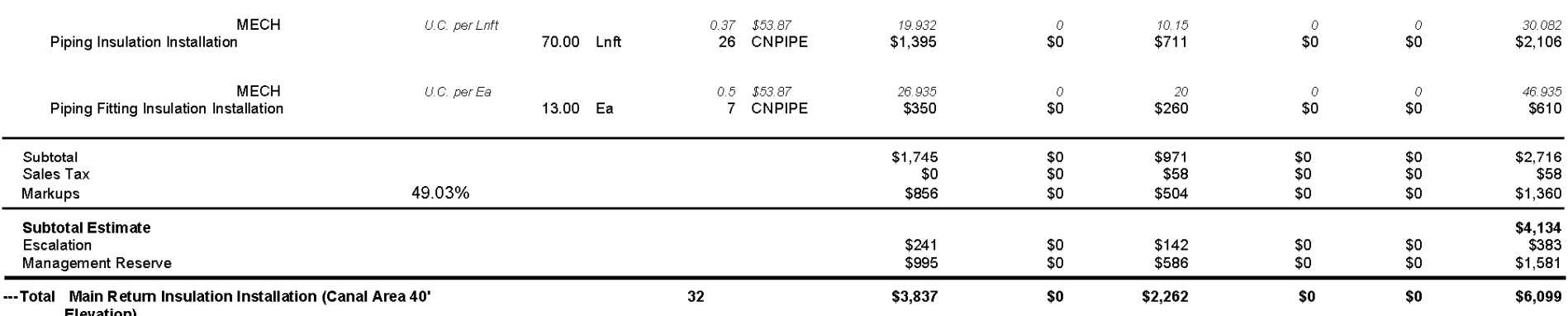

Elevation) 
Project Name: RTC Waste Heat Recovery

Project Location: ATR

Pstimate Number: 7850

Code Description contractor

overnead working Equilpment

This is a monthly cost

Snorkel Lit (2 Each To Spread TECH

U. . per Month 2.00 Month 4553.87

$\begin{array}{lrr}215.48 & 5370 \\ \text { CNPIPE } & \$ 431 & \$ 10,740\end{array}$

5370
0,740

Subtotal

Subtotal Estimat

Escalation

--Total Overhead Working Equipmen

\section{Main Supply Installation (West Canal Area 30' Elevation)}

$$
\begin{array}{ccc}
\text { MECH } & \text { U. per Lnit } & \\
\text { Overhead Metal Piping 8" Installation Supply } & & \text { Lnft }
\end{array}
$$

Piping (Canal Area)

8" Piping Flange Fitting Installation

Memo: RSMeans 221113.47

8" 90 Degree Elbow Pipe Fitting Installatio

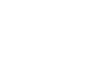

$3.00 \mathrm{Ea}$

$\mathrm{MECH}$
8" 45 Degree Elbow Pipe Fitting Installation

U.C. per Ea

$1.00 \quad \mathrm{Ea}$

$2.00 \mathrm{Ea}$

eans 221113.47 $\begin{array}{ll}1.27 & \$ 53.87 \\ 89 & \text { CNPIPE }\end{array}$

68.415
$\$ 4,789$

$\begin{array}{ll}4.8 & 553.87 \\ 14 & \text { CNPIPE }\end{array}$

$\begin{array}{ll}9 & \$ 53.87 \\ 9 & \text { CNPIPE }\end{array}$

$\begin{array}{ll}9 & 53.87 \\ 18 & \text { CNPIPE }\end{array}$

484.83
$\$ 970$

Client:

C. P. Ischay

Estimate Ty. A.W. Miller/S. N. Wasley

Equipment Material Subcontractor Other TOTAL

\begin{tabular}{rrrllr}
$\$ 431$ & $\$ 10,740$ & $\$ 0$ & $\$ 0$ & $\$ 0$ & $\$ 11,171$ \\
$\$ 0$ & $\$ 0$ & $\$ 0$ & $\$ 0$ & $\$ 0$ & $\$ 0$ \\
$\$ 211$ & $\$ 5,266$ & $\$ 0$ & $\$ 0$ & $\$ 0$ & $\$ 17$ \\
\hline
\end{tabular}

$\begin{array}{rrrrrr}\$ 60 & & & & \$ 16,648 \\ \$ 246 & \$ 1,484 & \$ 0 & \$ 0 & \$ 0 & \$ 1,543 \\ \$ 6,121 & \$ 0 & \$ 0 & \$ 0 & \$ 6,367 \\ \$ 947 & \$ 29,19 & & \$ 0 & \$ 0\end{array}$

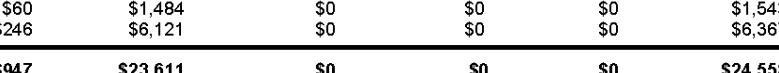

$\$ 24,558$

11:08:15
Cost Estimating 
Project Name: RTC Waste Heat Recovery

Project Location: ATR

\section{Code Description contractor}

Mlain Suppiy Installation (Wvest Canal Area 30 Elevation)
DETAIL ITEM REPORT

Client: C.P.Ischay

Prepared By: A.W. Miller/S. N. Wasley

Estimate Type: Class-5

Subcontractor Other TOTAL

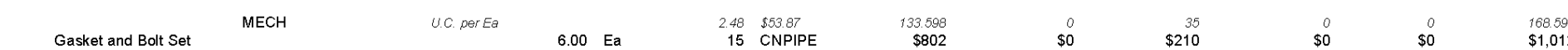

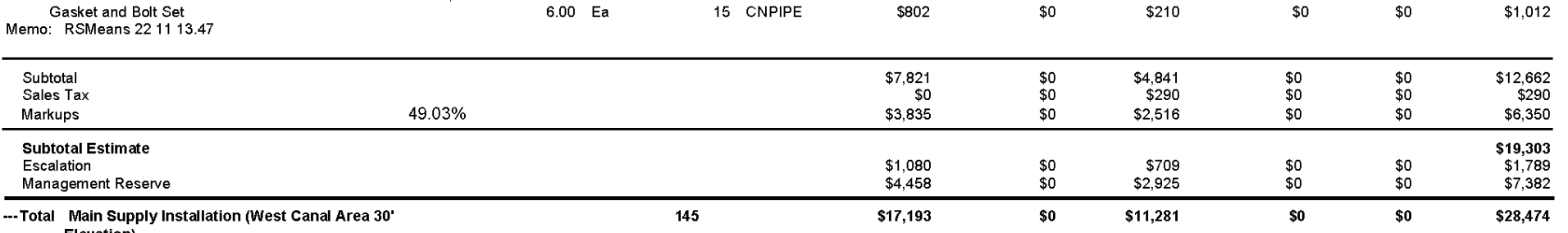

Main Return Installation (West Canal Area 30' Elevation) $\begin{array}{ccc}\text { MECH } & \text { U.C. per Lnit } \\ \text { Overhead Metal Piping 8" Installation Supply } & 70.00 \quad \text { Lnft }\end{array}$

Piping (Canal Area)
Memo: RSMeans 221113.44 Pipe Steel This has been factored $55 \%$ for the congested area

8" Piping Flange Fitting Installation
Memo: RSMeans 221113.47
8" 90 Degree Elbow Pipe FECH Fitting Installation
Merriv. RSMearirs 221113.47

8" 45 Degree Elbow Pipe Fitting Installation
Memo: RSMeans 221113.47

U.C. por Ea

$3.00 \mathrm{Ea}$

U. . per Ea

U.C. per Es

$1.00 \quad \mathrm{Ea}$

$2.00 \mathrm{Ea}$ $\begin{aligned} 1.27 & \$ 53.87 \\ 89 & \text { CNPIPE }\end{aligned}$

68.415
$\$ 4,789$

$4.8 \quad 553.87$

$9 \quad \$ 53.87$

$\begin{array}{ll}9 & 553.87 \\ 9 & \text { CNPIPE }\end{array}$

$\begin{aligned} 9 & \$ 53.87 \\ 18 & \text { CNPIPE }\end{aligned}$

484.83
$\$ 970$

484.83
$\$ 485$
$\$ 0$

$\$ 3,850$

$\$ 200$

0
$\$ 0$$\quad \$ 326$ $\begin{array}{lll}\$ 0 & 0 & \\ \$ 0 & \$ 23,415 & \$ 8,639\end{array}$

$\begin{array}{lll}0 & 0 & 343.577 \\ \$ 0 & \$ 0 & \$ 1,031\end{array}$

sin

$\begin{array}{lrr}0 & 0 & 68483 \\ \$ 0 & \$ 0 & \$ 685\end{array}$

$\sqrt{63}$

$\begin{array}{llr}0 & 0 & 6478 \\ & \$ 0 & \$ 1,296\end{array}$
5/11/2012 11:08:15 
Project Name: RTC Waste Heat Recovery

Project Location: ATR

Estimate Number:7850

\section{Code Description contractor}

Whain Retum Installation (w)est Canal Area 30" Elevation)

DETAIL ITEM REPORT

Prepared By: A.W. Miller/S. N. Wasley
Estimate Type: Class-5

Subcontractor Other TOTAL

\begin{tabular}{|c|c|c|c|c|c|c|c|c|c|}
\hline $\begin{array}{l}\text { Gasket and Bolt Set } \\
\text { Memo: RSMeans } 221113.47\end{array}$ & UC. per Ea & $6.00 \mathrm{Ea}$ & $\begin{aligned} 2.48 & \$ 53.87 \\
15 & \text { CNPIPE }\end{aligned}$ & $\begin{array}{r}\$ 33.508 \\
\$ 802\end{array}$ & $\begin{array}{l}0 \\
\$ 0\end{array}$ & $\begin{array}{r}35 \\
\$ 210\end{array}$ & $\$ 0$ & $\begin{array}{r}0 \\
\$ 0\end{array}$ & $\begin{array}{l}\$ 68.598 \\
\$ 1,012\end{array}$ \\
\hline $\begin{array}{l}\text { Subtotal } \\
\text { Sales Tax } \\
\text { Markups }\end{array}$ & $49.03 \%$ & & & $\begin{array}{r}\$ 7,821 \\
\$ 0 \\
\$ 3,835\end{array}$ & $\begin{array}{l}\$ 0 \\
\$ 0 \\
\$ 0\end{array}$ & $\begin{array}{r}\$ 4,841 \\
\$ 290 \\
\$ 2,516\end{array}$ & $\begin{array}{l}\$ 0 \\
\$ 0 \\
\$ 0\end{array}$ & $\begin{array}{l}\$ 0 \\
\$ 0 \\
\$ 0\end{array}$ & $\begin{array}{r}\$ 12,662 \\
\$ 290 \\
\$ 6,350\end{array}$ \\
\hline $\begin{array}{l}\text { Subtotal Estimate } \\
\text { Escalation } \\
\text { Management Reserve }\end{array}$ & & & & $\begin{array}{l}\$ 1,080 \\
\$ 4,458\end{array}$ & $\begin{array}{l}\$ 0 \\
\$ 0\end{array}$ & $\begin{array}{r}\$ 709 \\
\$ 2,925\end{array}$ & $\begin{array}{l}\$ 0 \\
\$ 0\end{array}$ & $\begin{array}{l}\$ 0 \\
\$ 0\end{array}$ & $\begin{array}{r}\$ 19,303 \\
\$ 1,789 \\
\$ 7,382\end{array}$ \\
\hline --Total Main Retum Installatio & on (West Canal Area 30' & & 45 & $\$ 17,193$ & $\$ 0$ & $\$ 11,281$ & $\$ 0$ & $\$ 0$ & $\$ 28,474$ \\
\hline
\end{tabular}

Main Supply Insulation Installation (West Canal Area $30^{\prime}$ Elevation)

Memo: RSMeans 220719.30

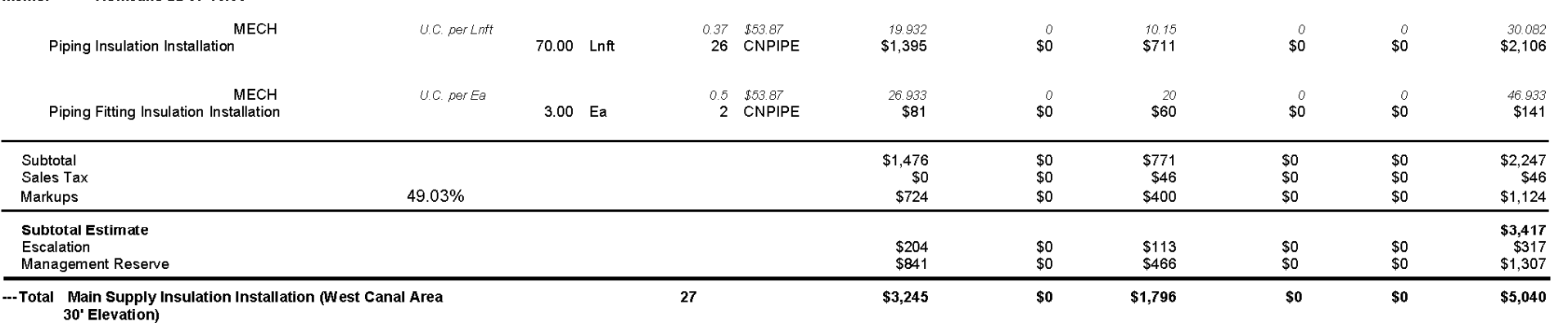

BEA

5/11/2012 11:08:15
Cost Estimating
Material Costs where applicable include Idaho State Sales Tax Page No. $\quad 52$ 
Project Name: RTC Waste Heat Recovery

Project Location: ATR

Pste 7850

\section{Code Description $\quad$ Contractor}

Vyain Retum Insulaulon Installation (west Canal Area 30. Elevation

Memo: RSMeans 220719.30

\begin{tabular}{|c|c|c|c|c|c|c|c|c|c|c|c|}
\hline Piping Insulation Installation ${ }^{\mathrm{MECH}}$ & U.c. per Lnit & 70.00 & Lnft & $\begin{array}{r}0.37 \\
26\end{array}$ & $\begin{array}{l}\$ 53.87 \\
\text { CNPIPE }\end{array}$ & $\begin{array}{r}19.932 \\
\$ 1,395\end{array}$ & $\begin{array}{r}0 \\
\$ 0\end{array}$ & $\begin{array}{l}10,15 \\
\$ 711\end{array}$ & $\begin{array}{c}0 \\
\text { \$o }\end{array}$ & \$o & $\begin{array}{r}30.082 \\
\$ 2,106\end{array}$ \\
\hline Piping Fitting Insulation In stallation & U.C. per Ea & & $\mathrm{Ea}$ & $\begin{array}{r}0.5 \\
2\end{array}$ & $\begin{array}{l}\$ 53.87 \\
\text { CNPIPE }\end{array}$ & $\begin{aligned} 26.933 \\
\$ 81\end{aligned}$ & $\begin{array}{l}0 \\
\$ 0\end{array}$ & $\begin{array}{r}20 \\
\$ 60\end{array}$ & $\begin{array}{r}0 \\
\$ 0\end{array}$ & $\begin{array}{l}0 \\
\$ 0\end{array}$ & $\begin{array}{l}46.933 \\
\$ 141\end{array}$ \\
\hline $\begin{array}{l}\text { Subtotal } \\
\text { Sales Tax } \\
\text { Markups }\end{array}$ & $49.03 \%$ & & & & & $\begin{array}{r}\$ 1,476 \\
\$ 0 \\
\$ 724\end{array}$ & $\begin{array}{l}\$ 0 \\
\$ 0 \\
\$ 0\end{array}$ & $\begin{array}{r}\$ 771 \\
\$ 46 \\
\$ 400\end{array}$ & $\begin{array}{l}\$ 0 \\
\$ 0 \\
\$ 0\end{array}$ & $\begin{array}{l}\$ 0 \\
\$ 0 \\
\$ 0\end{array}$ & $\begin{array}{r}\$ 2,247 \\
\$ 46 \\
\$ 1,124\end{array}$ \\
\hline $\begin{array}{l}\text { Subtotal Estimate } \\
\text { Escalation } \\
\text { Management Reserve }\end{array}$ & & & & & & $\$ 204$ & $\begin{array}{l}\$ 0 \\
\$ 0\end{array}$ & $\begin{array}{l}\$ 113 \\
\$ 466\end{array}$ & $\begin{array}{l}\$ 0 \\
\$ 0\end{array}$ & $\begin{array}{l}\$ 0 \\
\$ 0\end{array}$ & $\begin{array}{r}\$ 3,417 \\
\$ 317 \\
\$ 1,307\end{array}$ \\
\hline
\end{tabular}

\section{DETAIL ITEM REPORT}

lient: C. P. Ischay

Prepared By: A.W.Miller/S. N. Wasley

Estimate Type: Class-5

Qty UOM Hrs Resource Labor Equipment Material Subcontractor Other TOTAL

Qty UOM Hrs Resource Labor

Overhead Working Equipment

Memo: This is a momthly cost for two snorkel Iffts obtained from the Rental Rate Blue Book for Construction Equipment

\begin{tabular}{|c|c|c|c|c|c|c|c|c|c|}
\hline $\begin{array}{l}\text { MECH } \\
\text { Snorkel Lit ( } 2 \text { Each To Spread The Load Of The } \\
\text { Metal Piping) }\end{array}$ & U.C. per Month & 1.00 Month & $\begin{array}{l}4 \\
4\end{array}$ & \$o & $\begin{array}{r}5370 \\
\$ 5,370\end{array}$ & \$o & $\$$ & $\begin{array}{l}0 \\
\$ 0\end{array}$ & $\begin{array}{r}5370 \\
\$ 5,370\end{array}$ \\
\hline $\begin{array}{l}\text { Subtotal } \\
\text { Sales Tax } \\
\text { Markups }\end{array}$ & $49.03 \%$ & & & $\begin{array}{l}\$ 0 \\
\$ 0 \\
\$ 0\end{array}$ & $\begin{array}{r}\$ 5,370 \\
\$ 0 \\
\$ 2,633 \\
\end{array}$ & $\begin{array}{l}\$ 0 \\
\$ 0 \\
\$ 0\end{array}$ & $\begin{array}{l}\$ 0 \\
\$ 0 \\
\$ 0\end{array}$ & $\begin{array}{l}\$ 0 \\
\$ 0 \\
\$ 0\end{array}$ & $\begin{array}{r}\$ 5,370 \\
\$ 0 \\
\$ 2,633 \\
\end{array}$ \\
\hline $\begin{array}{l}\text { Subtotal Estimate } \\
\text { Escalation } \\
\text { Management Reserve }\end{array}$ & & & & $\begin{array}{l}\$ 0 \\
\$ 0\end{array}$ & $\begin{array}{r}\$ 742 \\
\$ 3,061\end{array}$ & $\begin{array}{l}\$ 0 \\
\$ 0\end{array}$ & $\begin{array}{l}\$ 0 \\
\$ 0\end{array}$ & $\begin{array}{l}\$ 0 \\
\$ 0\end{array}$ & $\begin{array}{r}\$ 8,003 \\
\$ 742 \\
\$ 3,061\end{array}$ \\
\hline Overl & & & & \$0 & $\$ 11,805$ & \$o & \$0 & \$0 & $\$ 11,805$ \\
\hline
\end{tabular}


Project Name: RTC Waste Heat Recovery

Project Location: ATR

Estimate Number:7850

\section{Code Description contractor}

\section{Maln Suppiv installation (west Canal Area $40^{\circ}$ Elevation)}

30.00 Lnft

Memo: RSMeans 221113.44 Pipe Steel This has been factored 55\% for the congested area

MECH
8" Piping Flange Fitting Installation
Memo. RSMeans 221113.47

$$
\text { U.C. per Ea }
$$

$1.00 \mathrm{Ea}$

8" 90 Degree Elbow Pipe Fitting Installation

U.c. per Ea

$\begin{array}{llll}1.00 \mathrm{Ea} & 9 & 553.87 \\ & 9 & \text { CNPIPE }\end{array}$

$\$ 2,052$

Equipment

Client: $\quad$ C. P. Ischay

Prepared By. A.W.Miller/S. N. Wasley

Estimate Type: Class-5

Memo: RSMeans 221113.47 .

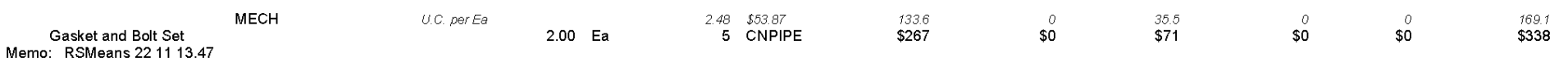

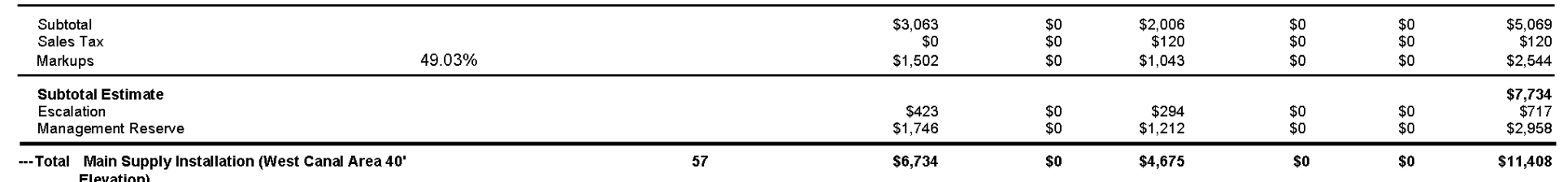

\section{Main Retum Install ation (west Canal Area 40. Elevation)}

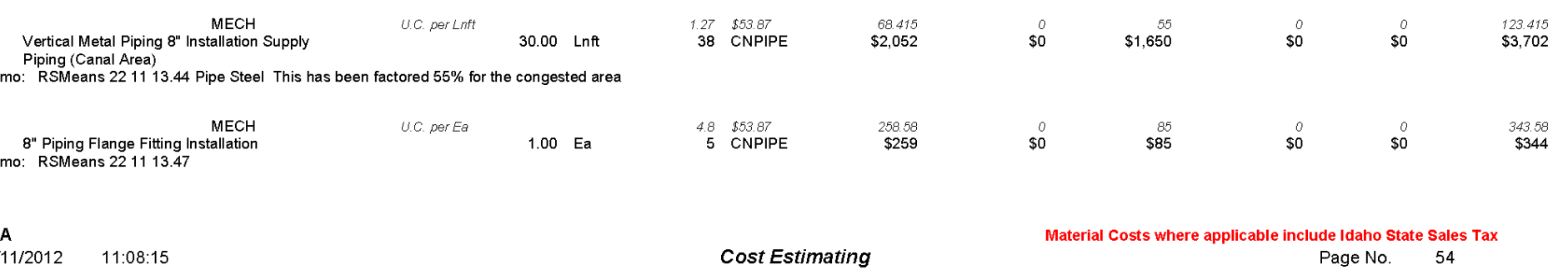


Project Name: RTC Waste Heat Recovery

Project Location: ATR

\section{Code Description $\quad$ contractor}

Mlain Retum installation (w)est Canal Area 40 Elevation)

DETAIL ITEM REPORT

client: C. P.Ischay

Prepared By: A. W. Miller/S. N. Wasley

Estimate Type: Class-5

Qty UOM Hrs Resource Labor Equipment Material Subcontractor Other TOTAL

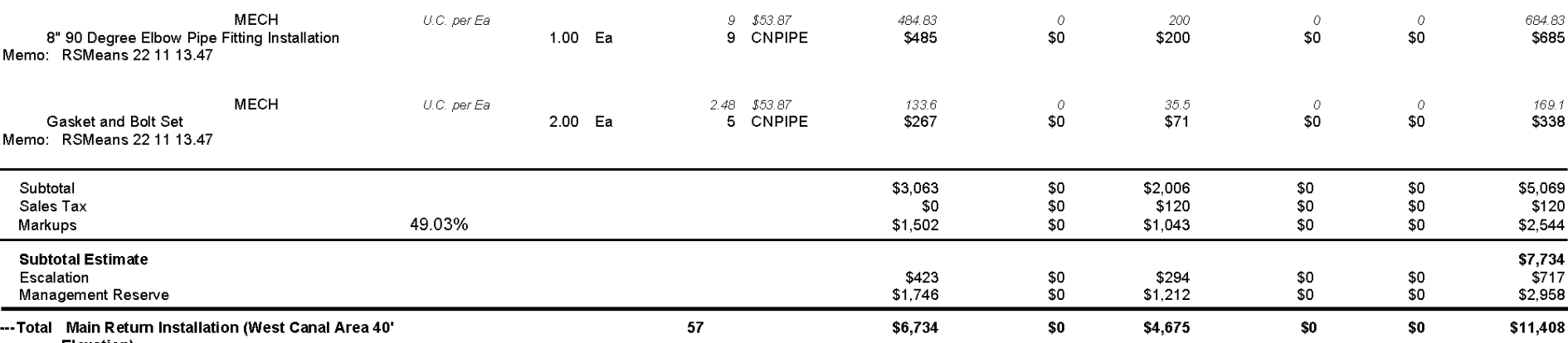

\section{Main Supply Insulation Installation (West Canal Area $40^{\circ}$ Elevation} Memo: RSMeans 220719.30
Piping Insulation InstallatioCH

U.c. per Lnit

$30.00 \quad$ Ln

$\begin{aligned} 0.37 & \$ 53.87 \\ 11 & \text { CNPIPE }\end{aligned}$

19.932
$\$ 598$

0
$\$ 0$

10.15
$\$ 305$

\$0

$\$$

30.082
$\$ 902$ 
Main Retum Insulation Installation (West Canal Area 40' Elevation)

Memo: RSMeans 220719.30

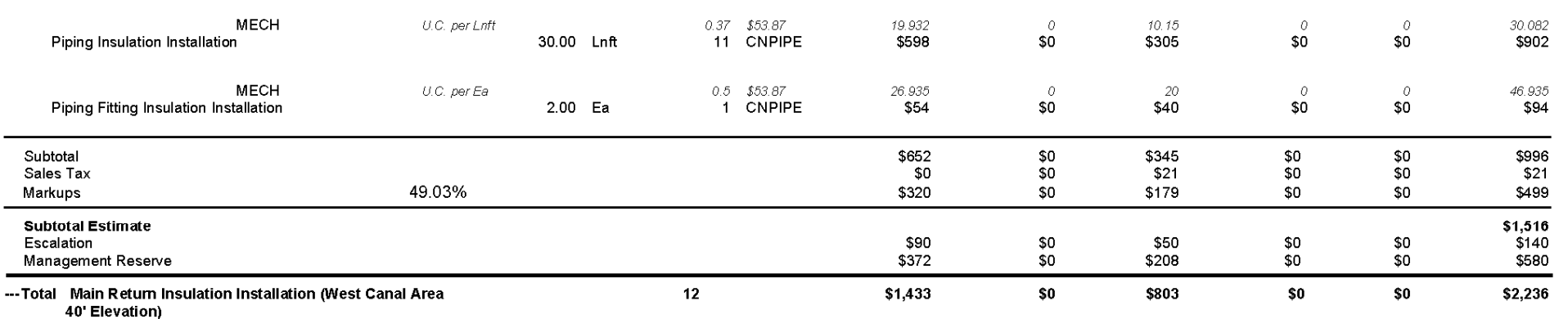

$40^{\prime}$ Elevation)

BEA

11:08:15
Project Name: RTC Waste Heat Recovery

Project Location: ATR

Code Description Contractor

Maaln Suppiv insulation Installation west Canal Area $40^{\circ}$ Elevation

Memo: $\quad$ RSMeans 220719.30

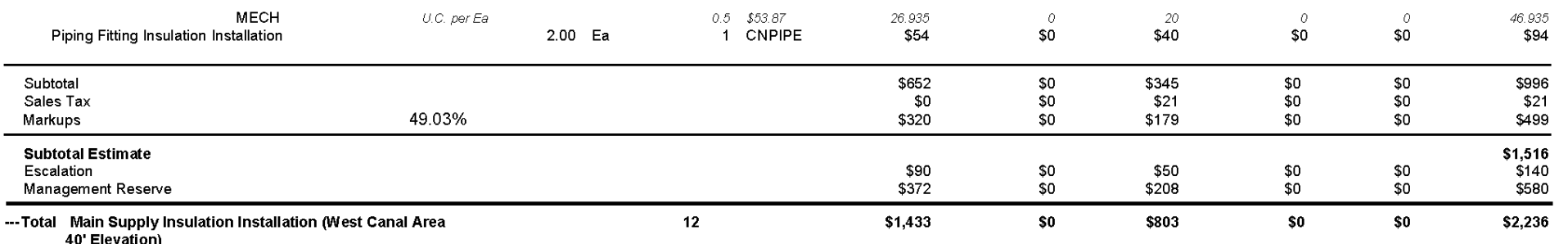

DETAIL ITEM REPORT

Client: C. P. Ischay

Prepared By: A. W. Miller/S. N. Wasley
Estimate Type: Class-5

Qty UOM Hrs Resource Labor Equipment Material Subcontractor Other TOTAL

Total Main Supply Insulation Installation (West Canal Area
$40^{\prime}$ Elevation)

12 
Project Name: RTC Waste Heat Recovery

Project Location: ATR

Pstimate Number: 7850

Code Description $\quad$ contractor

overnead working Equipment

This is a monthly cost

\section{MECH}

MECH
Snorkel Lit ( 2 Each To Spread The Load Of The U.C. per Month
Metal Piping)

Subtotal

Subtotal Estimate

Escalation
Management Reserv

--Total Overhead Working Equipmen

DETAIL ITEM REPORT

C.P. Ischay

Prepared By: A.W. Miller/S. N. Wasle
Estimate Type: Class-5

Qty UOM Hrs Resource Labor Equipment Material Subcontractor Other TOTAL

Main Supply Installation (Canal Area 30' Elevation)

$\mathrm{MECH}$
Overhead Metal Piping 8 "Installation Supply

U.C. per Lnit 70.00 Lnft

$\begin{array}{ll}1.27 & \$ 53.87 \\ 89 & \text { CNPIPE }\end{array}$

68.415
$\$ 4,789$

5370
$\$ 0$

$\begin{array}{rrrr}\$ 0 & 0 & 0 & 5370 \\ & \$ 0 & \$ 0 & \$ 5,370\end{array}$

RSMeans 221113.44 Pipe Steel This has been factored $55 \%$ for the congested area

MECH
Memo: PSMing Flange Fitting Installatio

U.c. per Ea

$1.00 \mathrm{Ea}$

$\begin{aligned} 4.8 & \$ 53.87 \\ 5 & \text { CNPIPE }\end{aligned}$

258.58
$\$ 259$

$\$ 0$

\begin{tabular}{lll}
$\$ 0$ & $\$ 0$ & $\$ 5,370$ \\
$\$ 0$ & $\$ 0$ & $\$ 0$ \\
$\$ 0$ & $\$ 0$ & $\$ 2,633$ \\
\hline
\end{tabular}

MECH

MECH
8" 90 Degree Elbow Pipe Fitting Installation

UC. perEa

$8.00 \mathrm{Ea}$

$\begin{aligned} 9 & \$ 53.87 \\ 72 & \text { CNPIPE }\end{aligned}$

48483
$\$ 3,879$

$\$ 0 \quad \$ 85$

$\begin{array}{llr}\$ 0 & \$ 0 & \$ 8,003 \\ \$ 0 & \$ 0 & \$ 742\end{array}$

MECH

8" 45 Degree Elbow Pipe Fitting Installation
Memo: RSMeans 221113.47

U.c. perEa

$4.00 \mathrm{Ea}$

$\begin{aligned} 9 & \$ 53.87 \\ 36 & \text { CNPIPE }\end{aligned}$

484.83
$\$ 1,939$

MECH
8" Tee Straight Pipe Fiting Installation
Memo: RSMeans 221113.47

U.C. perEa

2.00

$\begin{array}{rl}13.44 & \$ 53.87 \\ 27 & \mathrm{CNPIPE}\end{array}$

724.015
$\$ 1,448$

200
$\$ 0$

200
$\$ 1,600$

$\begin{array}{rr}0 & 163 \\ \$ 0 & \$ 652\end{array}$

$\$ 652$

$\begin{array}{rrr}0 & \$ 0 & 64783 \\ \$ 0 & \$ 0 & \$ 2,591\end{array}$

BEA

15/11/2012 11:08:15

Cost Estimating
Material costs where applicable include Idaho State Sales Tax Page No.
${ }_{\$ 8,639}^{123445}$

343.58
$\$ 344$

68483
$\$ 5,479$ 4783
52,591

1079015
$\$ 2,158$ 
Project Name: RTC Waste Heat Recovery

Project Location: ATR

Pstimate Number: 7850

\section{Code Description Contractor}

Muain Suppiy Installation (Canal Area 30" Elevation) $\mathrm{MECH}$

Gasket and Bolt Set
Memo: RSMeans 221113.47

DETAIL ITEM REPORT

\begin{tabular}{|c|c|c|c|c|c|c|c|c|c|}
\hline $\begin{array}{l}\text { Gasket and Bolt Set } \\
\text { Memo: RSMeans } 221113.47\end{array}$ & UC. per Ea & $15.00 \mathrm{Ea}$ & $\begin{aligned} 2.48 & \$ 53.87 \\
37 & \text { CNPIPE }\end{aligned}$ & $\begin{array}{l}\$ 33,507 \\
\$ 2,004\end{array}$ & $\begin{array}{l}0 \\
\$ 0\end{array}$ & $\begin{array}{r}35.5 \\
\$ 533\end{array}$ & $\begin{array}{l}0 \\
\$ 0\end{array}$ & $\$ 0$ & $\begin{array}{l}169.097 \\
\$ 2,536\end{array}$ \\
\hline $\begin{array}{l}\text { Subtotal } \\
\text { Sales Tax } \\
\text { Markups }\end{array}$ & $49.03 \%$ & & & $\begin{array}{r}\$ 14,318 \\
\$ 0 \\
\$ 7,020\end{array}$ & $\begin{array}{l}\$ 0 \\
\$ 0 \\
\$ 0\end{array}$ & $\begin{array}{r}\$ 7,430 \\
\$ 446 \\
\$ 3,861\end{array}$ & $\begin{array}{l}\$ 0 \\
\$ 0 \\
\$ 0\end{array}$ & $\begin{array}{l}\$ 0 \\
\$ 0 \\
\$ 0\end{array}$ & $\begin{array}{r}\$ 21,747 \\
\$ 446 \\
\$ 10,881\end{array}$ \\
\hline $\begin{array}{l}\text { Subtotal Estimate } \\
\text { Escalation } \\
\text { Management Reserve }\end{array}$ & & & & $\begin{array}{l}\$ 1,978 \\
\$ 8,160\end{array}$ & $\begin{array}{l}\$ 0 \\
\$ 0\end{array}$ & $\begin{array}{l}\$ 1,088 \\
\$ 4,489\end{array}$ & $\begin{array}{l}\text { \$0 } \\
\$ 0\end{array}$ & $\begin{array}{l}\$ 0 \\
\$ 0\end{array}$ & $\begin{array}{r}\$ 33,074 \\
\$ 3,066 \\
\$ 12,649\end{array}$ \\
\hline --Total Main Supply Installa & on (Canal & & & $\$ 31,476$ & \$o & $\$ 17,313$ & $\$ 0$ & \$0 & $\$ 48,789$ \\
\hline
\end{tabular}

Main Retum Installation (Canal Area $30^{\circ}$ Elevation)

$$
\text { Overhead Metal Piping 8" MECH }
$$

U.C. per Lint 70.00 Lnft

Memo: RSMeans 221113.44 Pipe Steel This has been factored 55\% for the congested area

$$
\begin{aligned}
& \text { 8" Piping Flange Fitting Installation } \\
& \text { Memo: RSMeans } 221113.47 \\
& \text { 8" } 90 \text { Degree Elbow Pipe Fitting Installation } \\
& \text { Memo: RSMeans } 221113.47 \\
& \text { 8" } 45 \text { Degree Elbow Pipe Fitting Installation } \\
& \text { Memo: RSMeans } 221113.47 \\
& \text { 8" Tee Straight Pipe Fitting MECH }
\end{aligned}
$$$$
\text { UC. per Ea }
$$

$1.00 \quad \mathrm{Ea}$

$$
\text { U.C. per Ea }
$$

800

8" Tee Straight Pipe Fitting Installation
Memo: RSMeans 221113.47

Client: C.P.Ischay

Prepared By: A.W. Miller/S. N. Wasle

Estimate Type: Class-5

Equipment Material Subcontractor Other TOTAL

Hrs Resource Labor 
Memo: RSMeans 220719.30

\begin{tabular}{|c|c|c|c|c|c|c|c|c|c|c|}
\hline Piping Insulation Installation ${ }^{\mathrm{MECH}}$ & U.c. per Lnit & 70.00 & Lnft & $\begin{array}{ll}0.37 & \$ 53.87 \\
26 & \text { CNPIPE }\end{array}$ & $\begin{array}{r}19.932 \\
\$ 1,395\end{array}$ & $\stackrel{0}{\$ 0}$ & $\begin{array}{l}10.15 \\
\$ 711\end{array}$ & $\begin{array}{r}0 \\
\$ 0\end{array}$ & $\$$ & $\begin{array}{r}30.082 \\
\$ 2,106\end{array}$ \\
\hline $\begin{array}{r}\mathrm{MECH} \\
\text { Piping Fitting Insulation Installation }\end{array}$ & UC. perEa & 15.00 & $\mathrm{Ea}$ & $\begin{array}{rl}0.5 & 553.87 \\
8 & C N P I P E\end{array}$ & $\begin{array}{l}26.935 \\
\$ 404\end{array}$ & $\$ 0$ & $\begin{array}{r}20 \\
\$ 300\end{array}$ & $\stackrel{0}{\$ 0}$ & $\begin{array}{l}0 \\
\$ 0\end{array}$ & $\begin{array}{r}46.935 \\
\$ 704\end{array}$ \\
\hline $\begin{array}{l}\text { Subtotal Estimate } \\
\text { Escalation } \\
\text { Management Reserve }\end{array}$ & & & & & $\begin{array}{r}\$ 249 \\
\$ 1,025\end{array}$ & $\begin{array}{l}\$ 0 \\
\$ 0\end{array}$ & $\begin{array}{l}\$ 148 \\
\$ 610\end{array}$ & $\begin{array}{l}\$ 0 \\
\$ 0\end{array}$ & $\begin{array}{l}\$ 0 \\
\$ 0\end{array}$ & $\begin{array}{r}\$ 4,278 \\
\$ 397 \\
\$ 1,636\end{array}$ \\
\hline
\end{tabular}

---Total Main Supply Insulation Installation (Canal Area 30'

33

$\$ 3,955$

BEA

5/11/2012 11:08:15
Project Name: RTC Waste Heat Recovery

Project Location: ATR

stimate Number: 7850

\section{Code Description Contractor}

Mlain Return Installation (CCanal Area 30' Elevation)

MECH

Gasket and Bolt Set
Memo: RSMeans 221113.47

\begin{tabular}{l}
\hline $\begin{array}{l}\text { Subtotal } \\
\text { Sales Tax } \\
\text { Markups }\end{array}$ \\
\hline Subtotal Estimate \\
Escalation \\
Management Reserve \\
\hline- --Total Main Retum Installation (Canal Area 30' Elevation) \\
Main Supply Insulation Installation (Canal Area 30' Elevation)
\end{tabular}

DETAIL ITEM REPORT

Client: $\quad$ C.P. Ischa

Prepared By: A. W. Miller/S. N. Wasley
Estimate Type: Class-5

Qty UOM Hrs Resource Labor Equipment Material Subcontractor Other TOTAL

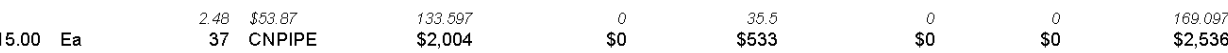

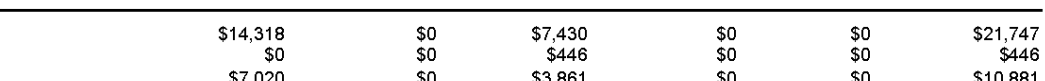

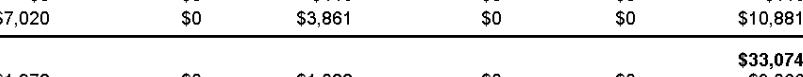

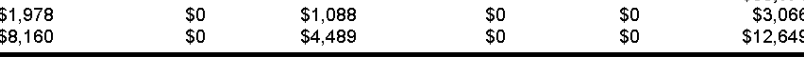

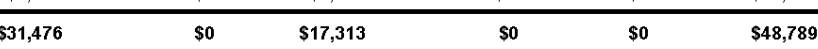


Project Name: RTC Waste Heat Recovery

Project Location: ATR

Estimate Number: 7850

\section{Code Description Contractor}

MMain Retum insulation installation (Canal Area 30 Elevation)

Memo: RSMeans 220719.30

\begin{tabular}{|c|c|c|c|c|c|c|c|c|c|}
\hline Piping Insulation Installation & U.C. per Lnit & 70.00 Lnft & $\begin{array}{ll}0.37 & \$ 53.87 \\
26 & \text { CNPIPE }\end{array}$ & $\begin{array}{r}19.932 \\
\$ 1,395\end{array}$ & \$o & $\begin{array}{l}10.15 \\
\$ 711\end{array}$ & $\begin{aligned} 0 \\
\text { \$o }\end{aligned}$ & \$o & $\begin{array}{r}30.082 \\
\$ 2,106\end{array}$ \\
\hline $\begin{array}{r}\mathrm{MECH} \\
\text { Piping Fitting Insulation Installation }\end{array}$ & UC. per Ea & $15.00 \mathrm{Ea}$ & $\begin{aligned} 0.5 & \$ 53.87 \\
8 & \text { CNPIPE }\end{aligned}$ & $\begin{array}{r}26.935 \\
\$ 404\end{array}$ & $\$$ & $\begin{array}{r}20 \\
\$ 300\end{array}$ & $\begin{array}{l}0 \\
\$ 0\end{array}$ & $\$ 0$ & $\begin{array}{r}46.935 \\
\$ 704\end{array}$ \\
\hline $\begin{array}{l}\text { Subtotal Estimate } \\
\text { Escalation } \\
\text { Management Reserve }\end{array}$ & & & & $\begin{array}{r}\$ 249 \\
\$ 1,025\end{array}$ & $\begin{array}{l}\$ 0 \\
\$ 0\end{array}$ & $\begin{array}{l}\$ 148 \\
\$ 610\end{array}$ & $\begin{array}{l}\$ 0 \\
\$ 0\end{array}$ & $\begin{array}{l}\$ 0 \\
\$ 0\end{array}$ & $\begin{array}{r}\$ 4,278 \\
\$ 397 \\
\$ 1,636\end{array}$ \\
\hline
\end{tabular}

\section{DETAIL ITEM REPORT}

Client: C.P. Ischay

Prepared By: A.W.Miller/S. N. Wasley

Estimate Type: Class-5

Subcontractor

Other TOTAL

Overhead Working Equipmen

Memo: This is a monthly cost for two snorkel lifts obtained from the Rental Rate Blue Book for Construction Equipment.

\begin{tabular}{|c|c|c|c|c|c|c|c|c|c|}
\hline $\begin{array}{l}\text { MECH } \\
\text { Snorkel Lit (2 Each To Spread The Load Of The } \\
\text { Metal Piping) }\end{array}$ & U.C. per Month & 1.00 Month & $\begin{array}{ll}4 & 553.87 \\
4 & \text { CNPIPE }\end{array}$ & $\begin{array}{r}\begin{array}{r}215.48 \\
\$ 215\end{array} \\
\end{array}$ & $\begin{array}{r}5370 \\
\$ 5,370\end{array}$ & $\$ 0$ & $\$ 0$ & $\$ 0$ & $\begin{array}{l}558548 \\
\$ 5,585\end{array}$ \\
\hline $\begin{array}{l}\text { Subtotal } \\
\text { Sales Tax } \\
\text { Markups }\end{array}$ & $49.03 \%$ & & & $\begin{array}{r}\$ 215 \\
\$ 0 \\
\$ 106\end{array}$ & $\begin{array}{r}\$ 5,370 \\
\$ 0 \\
\$ 2,633\end{array}$ & $\begin{array}{l}\$ 0 \\
\$ 0 \\
\$ 0\end{array}$ & $\begin{array}{l}\$ 0 \\
\$ 0 \\
\$ 0\end{array}$ & $\begin{array}{l}\$ 0 \\
\$ 0 \\
\$ 0\end{array}$ & $\begin{array}{r}\$ 5,585 \\
\$ 0 \\
\$ 2,739\end{array}$ \\
\hline $\begin{array}{l}\text { Subtotal Estimate } \\
\text { Escalation } \\
\text { Management Reserve }\end{array}$ & & & & $\begin{array}{l}\$ 30 \\
\$ 123\end{array}$ & $\begin{array}{r}\$ 742 \\
\$ 3,061\end{array}$ & $\begin{array}{l}\$ 0 \\
\$ 0\end{array}$ & $\begin{array}{l}\$ 0 \\
\$ 0\end{array}$ & $\begin{array}{l}\$ 0 \\
\$ 0\end{array}$ & $\begin{array}{r}\$ 8,324 \\
\$ 772 \\
\$ 3,183\end{array}$ \\
\hline
\end{tabular}


Project Name: RTC Waste Heat Recovery

Project Location: ATR

Estimate Number: 7850

\section{Code Description Contractor}

Whain Suppiv Installation (Canal Area 20" Elevation)

\section{DETAIL ITEM REPORT}

Client: C. P.Ischay

Prepared By: A.W. Miller/S. N. Wasley

Estimate Type: Class-5

Qty UOM Hrs Resource Labor Equipment Material Subcontractor Other TOTAL

MECH

MECH
8" Piping Flange Fitting Installation
Memo. RSMeans 221113.47

UC. perEa

$1.00 \mathrm{Ea}$

68.415
$\$ 5,473$

$\$ 0$

55

$\begin{array}{lll}48 & 553.87 & 258.58 \\ 5 & \text { CNPIPE } & 5259\end{array}$

$\begin{array}{rrr}258.58 & 0 & 85 \\ \$ 259 & \$ 0 & \$ 85\end{array}$

85
$\$ 85$

$\begin{array}{rlr}9 & \$ 53.87 & 484.83 \\ 36 & \text { CNPIPE } & \$ 1,939\end{array}$

8" 90 Degree Elbow Pipe Fitting Installation

UC. perEa

$4.00 \quad$ Ea

$\begin{array}{rr}0 & 200 \\ \$ 0 & \$ 800\end{array}$ $\begin{array}{rlr}9 & 553.87 & 484.83 \\ 36 & \text { CNPIPE } & \$ 1,939\end{array}$

$4.00 \mathrm{Ea}$

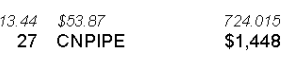

$2.00 \mathrm{Ea}$

$2.49 \quad 553.87$

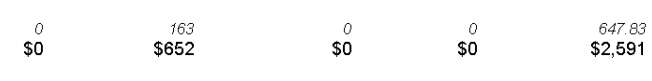

$\begin{array}{rrrrr}0 & 355 & 0 & 0 & 1079.075 \\ \$ 0 & \$ 710 & \$ 0 & \$ 0 & \$ 2,158\end{array}$

Gasket and Bolt Set
Memo: RSMeans 221113.47

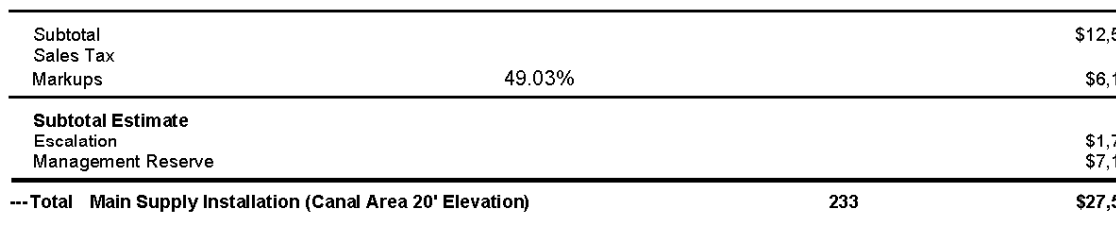

$\$ 12,528$

$\$ 6,142$

$\$ 1,731$

$\$ 27,542$

$\quad 35.5$

$\begin{array}{lrllr}\$ 0 & \$ 7,038 & \$ 0 & \$ 0 & \$ 19,566 \\ \$ 0 & \$ 422 & \$ 0 & \$ 0 & \$ 422 \\ \$ 0 & \$ 3,657 & \$ 0 & \$ 0 & \$ 9,800\end{array}$

\begin{tabular}{llllr}
$\$ 0$ & $\$ 0$ & $\$ 0$ & $\$ 9,800$ \\
$\$ 0$ & $\$ 1,031$ & $\$ 0$ & $\$ 0$ & $\$ 29,788$ \\
\hline 00 & $\$, 252$ & & $\$ 0$ & $\$ 1,7992$ \\
\hline
\end{tabular}

\begin{tabular}{lllll}
$\$ 0$ & $\$ 1,031$ & $\$ 0$ & $\$ 0$ & $\$ 29,788$ \\
$\$ 0$ & $\$ 4,252$ & $\$ 0$ & $\$ 0$ & $\$ 11,761$ \\
\hline
\end{tabular}

BEA

11:08:15
Cost Estimating
Material costs where applicable include Idaho State Sales Tax Page No. 6 
Project Name: RTC Waste Heat Recovery

Project Location: ATR

Estimate Number: 7850

\section{Code Description $\quad \underline{\text { Contractor }}$}

Whain Retum Installation (Canal Area 20* Elevation)

\section{DETAIL ITEM REPORT}

lient: C.P.Ischay

Prepared By: A. W. Miller/S. N. Wasley
Estimate Type: Class-5

Qty UOM Hrs Resource Labor Equipment Material Subcontractor Other TOTAL

Piping 8" Installation Supply

MECH
8" Piping Flange Fitting Installation
Memo: RSMeans 221113.47

UC. perEa

$1.00 \mathrm{Ea}$

68.415
$\$ 5,473$

$\$ 0$

55
$\$ 4,400$

55
4,400

$\$ 0$

$\$ 23,415$

(MECH

8" 90 Degree Elbow Pipe Fitting Installation

U.C. per Ea

$\begin{aligned} 4.8 & \$ 53.87 \\ 5 & \text { CNPIPE }\end{aligned}$

258.58
$\$ 259$

$\begin{array}{rr}0 & 85 \\ \$ 0 & \$ 85\end{array}$

85
$\$ 85$

$\begin{array}{rl}9 & \$ 53.87 \\ 36 & \mathrm{CNPIPE}\end{array}$

484.83
$\$ 1,939$

$\begin{array}{rr}0 & 200 \\ \$ 0 & \$ 800\end{array}$

$\begin{array}{rrr}0 & 0 & 34358 \\ \$ 0 & \$ 0 & \$ 344\end{array}$

\section{U.C. per Ea}

$4.00 \mathrm{Ea}$

$\begin{aligned} 9 & \$ 53.87 \\ 36 & \text { CNPIPE }\end{aligned}$

484.83
$\$ 1,939$

$\begin{array}{rr}0 & 163 \\ \$ 0 & \$ 652\end{array}$

$\begin{array}{rrr}0 & \$ & 684,83 \\ \$ 0 & \$ 0 & \$ 2,739\end{array}$

Memo: RSMeans 221113.47

$\begin{array}{rrr}0 & 0 & 647.83 \\ \$ 0 & \$ 0 & \$ 2,591\end{array}$

U.C. per Ea

$2.00 \mathrm{Ea}$

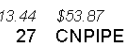

724.015
$\$ 1,448$

355
$\$ 0$$\quad \$ 710$

$\begin{aligned} 2.49 & \$ 53.67 \\ 27 & \text { CNPIPE }\end{aligned}$

$\$ 133.597$

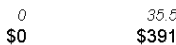

35.5
$\$ 391$

0

Memo: RSSM and Bolt Set

\begin{tabular}{|c|c|c|}
\hline $\begin{array}{l}\text { Subtotal } \\
\text { Sales Tax } \\
\text { Markups }\end{array}$ & $49.03 \%$ & \\
\hline $\begin{array}{l}\text { Subtotal Estimate } \\
\text { Escalation } \\
\text { Management Reserve }\end{array}$ & & $\$ 1$, \\
\hline
\end{tabular}

$\$ 12,52$
$\$ 0$
$\$ 6,142$

$\begin{array}{rlr}\$ 2,528 & \$ 0 & \$ 7,038 \\ \$ 0 & \$ 0 & \$ 422 \\ \$ 6,142 & \$ 0 & \$ 3,657\end{array}$

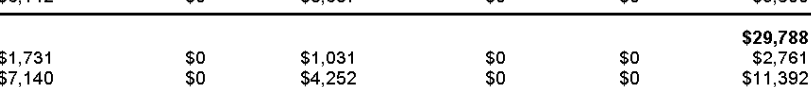

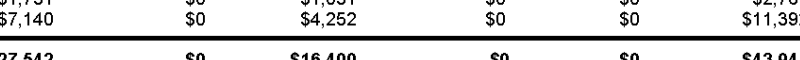

BEA

11:08:15 
Project Name: RTC Waste Heat Recovery

Project Location: ATR

Estimate Number: 7850

\section{Code Description $\quad$ contractor}

Mlaan Suppiv Insulation Installation (Canal Area 20' Elevation)

Memo: RSMeans 220719.30

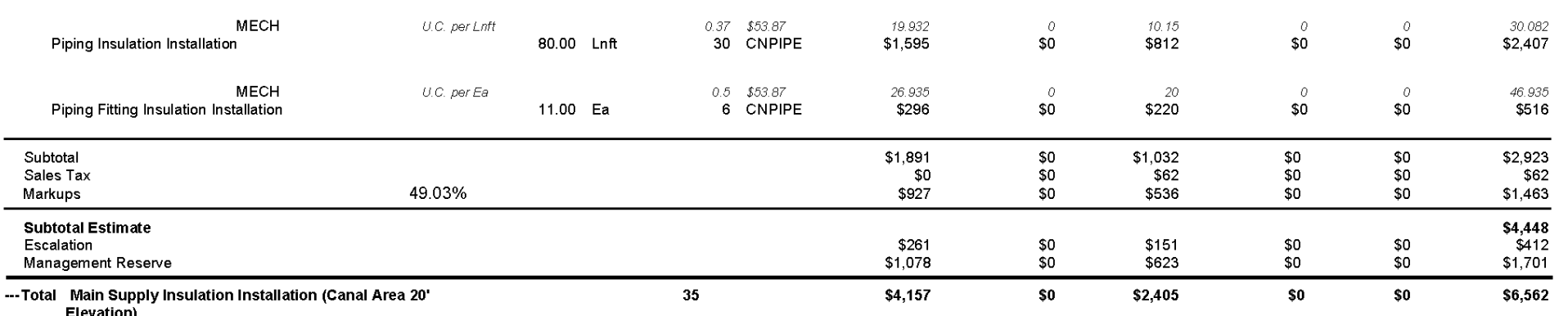

Main Retum Insulation Installation (Canal Area 20' Elevation)

Memo: RSMeans 220719.30

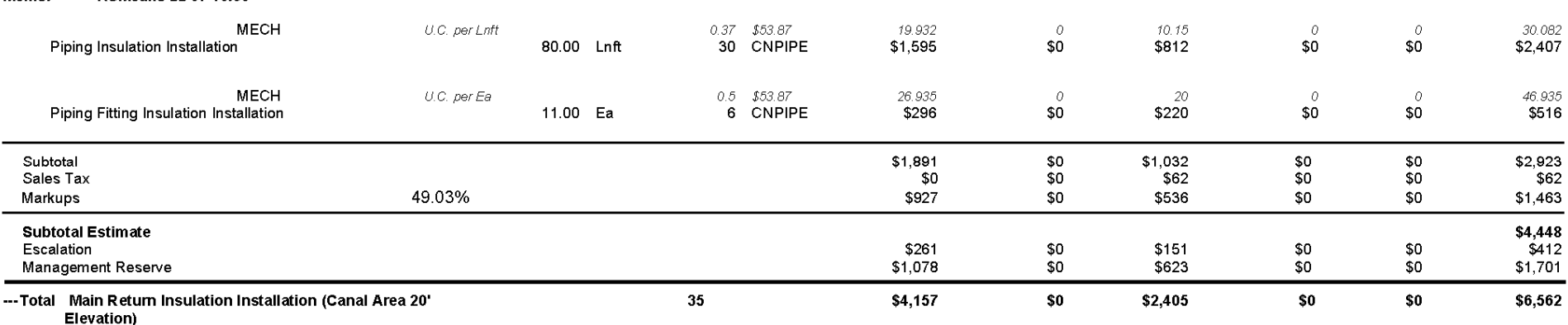

Main Retum Insulation Installation (Canal Area $20^{2}$

5/11/2012 11:08:15

\section{DETAIL ITEM REPORT}

Client: C.P.Ischay

Prepared By: A.W.Miller/S. N. Wasley

Estimate Type: Class-5

Qty UOM Hrs Resource Labor Equipment Material Subcontractor Other TOTAL

$\$ 4,157$

$\$ \$ \$ 1,701$ 
Project Name: RTC Waste Heat Recovery

Project Location: ATR

Pstimate Number: 7850

Code Description $\quad$ contractor

overnead working Equlpment

This is a monthly cost

\section{MECH UC. per Month $1.00 \mathrm{Moth}$}

Snorkel Lit (2 Each To Spreach Metal Piping

\begin{tabular}{lc}
\hline Subtotal \\
Sales Tax \\
Markups
\end{tabular}$\quad 49.03 \%$

Escalation
Management Reserve

--Total Overhead Working Equipment

DETAIL ITEM REPORT

lient: C.P.Ischay

Prepared By: A.W. Miller/S. N. Wasley

Estimate Type: Class-5

Qty UOM Hrs Resource Labor Equipment Material Subcontractor Other TOTAL

Main Supply Installation (Canal Area 40' Elevation)

MECH
Overhead Metal Piping Installation Supply Piping

U.C. per Lnit 140.00 Lnt

$\begin{aligned} 0.66 & \$ 53.87 \\ 92 & \text { CNPIPE }\end{aligned}$

35.554
$\$ 4,978$

$\$ 0$

4" (Canal Area)
. RSMeans 2211 .44 Pipe Steel This has been factored 55\% for the congested area

MECH
Piping Flange Fitting 4", Installation
Memo: RSMeans 221113.47

U.c. per Ea

$3.00 \quad \mathrm{Ea}$

$\begin{aligned} 2.6 & \$ 53.87 \\ 8 & \text { CNPIPE }\end{aligned}$

140.063
$\$ 420$

$\$ 0$

MECH
4" - 90 Degree Elbow Pipe Fitting Installation
MerrIu. RSMEarils 221113.47

UC. perEa

$\begin{array}{rrrr}2.00 \mathrm{Ea} & 45 & 553.87 \\ & 9 & \mathrm{CNPIPE}\end{array}$

242.415
$\$ 485$

$\$$

4" - 45 Degree Elbow: Pipe Fitting Installation
Memo: RSMeans 221113.47

U.C. per Ea

$\begin{array}{llll}4.00 & \mathrm{Ea} & 4.5 & 553.87 \\ & 18 & \text { CNPIPE }\end{array}$

242.415
$\$ 970$

4" - Tee Straight Pipe Fitting Installation

2.00

$\begin{array}{ll}7.5 & \$ 53.87 \\ 15 & \text { CNPIPE }\end{array}$

404.025
$\$ 808$

$\begin{array}{lr}\$ 9 & 49.5 \\ \$ 0 & \$ 198\end{array}$

0
$\$ 0$$\quad \$ 236$

$\$ 236$

$\begin{array}{rrr}0 & 0 & 522.025 \\ \$ 0 & \$ 0 & \$ 1,044\end{array}$
70.554
$\$ 9,878$

519

$\$ 581$

291.915
$\$ 1,168$
11:08:15
Cost Estimating
Material costs where applicable include Idaho State Sales Tax Page No. 
Project Name: RTC Waste Heat Recovery

Project Location: ATR

Pstimate Number: 7850

\section{Code Description Contractor}

Mlain Suppiy Installation (Canal Area 40" Elevation) $\mathrm{MECH}$

Gasket and Bolt Set
Memo: RSMeans 221113.47

DETAIL ITEM REPORT

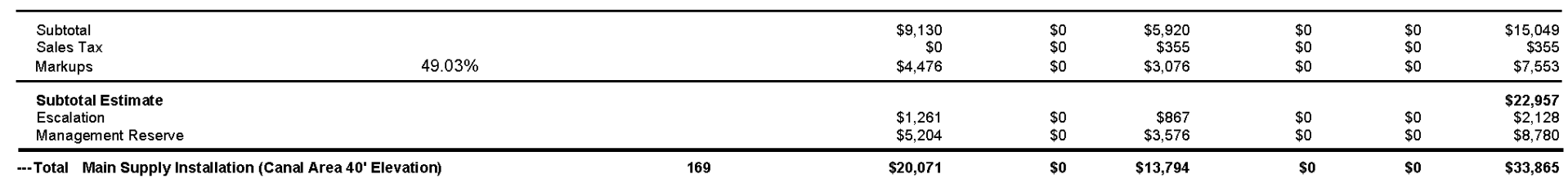

Client:

Prepared By: A.W. Miller/S. N. Wasle
Estimate Type: Class-5

Equipment Material Subcontractor Other TOTAL

1,860 355

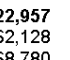
33,865

Main Retum Installation (Canal Area 40 Elevation)

MECH
Overhead Metal Piping Installation Supply Piping U.C. per Lnit
4" (Canal Area)
Memo: RSMeans 2211113.44 Pipe Steel This has been factored 55\% for the congested area

$$
\text { u. per Ea }
$$

$$
\text { U.C. perEa }
$$

U.C. per Ea

U.C. per Ea

MECH
4" - Tee Straight Pipe Fitting Installation
Memo. RSMeans 221113.47

BEA

11:08:15
$3.00 \mathrm{Ea}$

$2.00 \mathrm{Ea}$

$\begin{array}{rl}0.66 & 553.87 \\ 92 & \text { CNPIPE }\end{array}$

35.554
$\$ 4,978$

$\begin{aligned} 2.6 & \$ 53.87 \\ 8 & \text { CNPIPE }\end{aligned}$

$\begin{array}{rl}4.5 & 553.87 \\ 9 & \text { CNPIPE }\end{array}$

$\$ 40.063$
$\$ 420$

242.415
$\$ 485$

$\begin{array}{ll}4.5 & 553.87 \\ 18 & \text { CNPIPE }\end{array}$

$\begin{array}{ll}7.5 & 553.87 \\ 15 & \text { CNPIPE }\end{array}$

$2.00 \mathrm{Ea}$

$\begin{array}{llll} & 4.5 & 553.87 & 242 \\ 4.00 \mathrm{Ea} & 18 & \text { CNPIPE } & \\ & & & \\ & & & \end{array}$


Project Name: RTC Waste Heat Recovery

Project Location: ATR

stimate Number: 7850

\section{Code Description Contractor}

Malain Retum Installation (Canal Area 40.' Elevation)

MECH

Gasket and Bolt Set
Memo. RSMeans 221113.47

\begin{tabular}{l}
\hline $\begin{array}{l}\text { Subtotal } \\
\text { Sales Tax } \\
\text { Markups }\end{array}$ \\
\hline Subtotal Estimate \\
Escalation \\
Management Reserve \\
\hline- --Total Main Retum Installation (Canal Area 40' Elevation) \\
Main Supply Insulation Installation (Canal Area 40' Elevation)
\end{tabular}

Memo: $\quad$ RSMeans 220719.30

\begin{tabular}{|c|c|c|c|c|c|c|c|c|c|c|c|}
\hline Piping Insulation Installation & U. per Lnit & 140.00 & Lnft & $\begin{array}{r}0.37 \\
52\end{array}$ & $\begin{array}{l}\$ 53.87 \\
\text { CNPIPE }\end{array}$ & $\begin{array}{r}19.932 \\
\$ 2,790\end{array}$ & \$o & $\begin{array}{r}10.15 \\
\$ 1,421\end{array}$ & $\begin{array}{r}0 \\
\$ 0\end{array}$ & \$o & $\begin{array}{r}30.082 \\
\$ 4,211\end{array}$ \\
\hline $\begin{array}{r}\mathrm{MECH} \\
\text { Piping Fitting Insulation Installation }\end{array}$ & UC. perEa & 11.00 & $\mathrm{Ea}$ & $\begin{array}{r}0.5 \\
6\end{array}$ & $\begin{array}{l}553.87 \\
\text { CNPIPE }\end{array}$ & $\begin{array}{r}26.935 \\
\$ 296\end{array}$ & \$o & $\$ 220$ & $\begin{array}{r}0 \\
\$ 0\end{array}$ & $\$ 0$ & $\begin{array}{r}46.935 \\
\$ 516\end{array}$ \\
\hline $\begin{array}{l}\text { Subtotal Estimate } \\
\text { Escalation } \\
\text { Management Reserve }\end{array}$ & & & & & & $\begin{array}{r}\$ 426 \\
\$ 1,759\end{array}$ & $\begin{array}{l}\$ 0 \\
\$ 0\end{array}$ & $\begin{array}{l}\$ 240 \\
\$ 991\end{array}$ & $\begin{array}{l}\$ 0 \\
\$ 0\end{array}$ & $\begin{array}{l}\$ 0 \\
\$ 0\end{array}$ & $\begin{array}{l}\$ 7,192 \\
\$ 667 \\
\$ 2,751\end{array}$ \\
\hline
\end{tabular}

---Total Main Supply Insulation Installation (Canal Area 40'

57

$\$ 6,786$

$\$ 0$

BEA

5/11/2012 11:08:15

DETAIL ITEM REPORT

lient: C.P.Ischay

Prepared By: A.W.Miller/S. N. Wasley

Estimate Type: Class-5

Qty UOM Hrs Resource Labor Equipment Material Subcontractor Other TOTAL

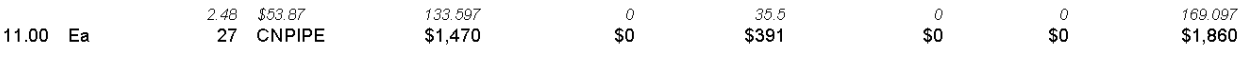

$\begin{array}{rrrrrr}\$ 9,130 & \$ 0 & \$ 5,920 & \$ 0 & \$ 0 & \$ 15,049 \\ \$ 0 & \$ 0 & \$ 355 & \$ 0 & \$ 0 & \$ 355 \\ 54,470 & \$ 0 & \$ 3,070 & \$ 0 & \$ 0\end{array}$

$\$ 0 \quad \$ 7,553$

\begin{tabular}{rrrrrr}
$\$ 1,261$ & $\$ 0$ & $\$ 867$ & $\$ 0$ & $\$ 0$ & $\$ 22,957$ \\
$\$ 5,204$ & $\$ 0$ & $\$ 3,576$ & $\$ 0$ & $\$ 0$ & $\$ 8,128$ \\
\hline 20,071 & $\$ 0$ & $\$ 13,794$ & $\$ 0$ & $\$ 0$ & $\$ 33,865$
\end{tabular}

169 
Project Name: RTC Waste Heat Recovery

Project Location: ATR

Estimate Number: 7850

\section{Code Description Contractor}

MMain Retum insulation installation (Canal Area 40. Elevation)

Memo: RSMeans 220719.30

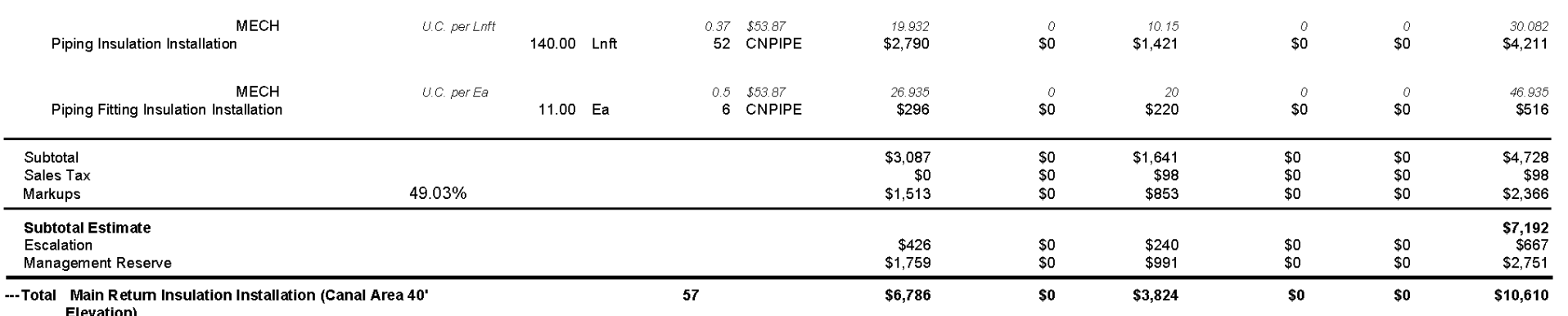

Overhead Working Equipment

Memo: This is a monthly cost for two snorkel Iffs obtained from the Rental Rate Blue Book for Construction Equipment.

\begin{tabular}{|c|c|c|c|c|c|c|c|c|c|}
\hline $\begin{array}{l}\text { MECH } \\
\text { Snorkel Litt (2 Each To Spread The Load Of The } \\
\text { Metal Piping) }\end{array}$ & UC. per Month & 1.00 Month & $\begin{array}{ll}4 & \$ 53.87 \\
4 & \text { CNPIPE }\end{array}$ & $\begin{array}{r}215.48 \\
\$ 215\end{array}$ & $\begin{array}{r}5370 \\
\$ 5,370\end{array}$ & \$o & \$o & $\$ 0$ & $\begin{array}{l}555548 \\
\$ 5,585\end{array}$ \\
\hline $\begin{array}{l}\text { Subtotal } \\
\text { Sales Tax } \\
\text { Markups }\end{array}$ & $49.03 \%$ & & & $\begin{array}{r}\$ 215 \\
\$ 0 \\
\$ 106\end{array}$ & $\begin{array}{r}\$ 5,370 \\
\$ 0 \\
\$ 2,633\end{array}$ & $\begin{array}{l}\$ 0 \\
\$ 0 \\
\$ 0\end{array}$ & $\begin{array}{l}\$ 0 \\
\$ 0 \\
\$ 0\end{array}$ & $\begin{array}{l}\$ 0 \\
\$ 0 \\
\$ 0\end{array}$ & $\begin{array}{r}\$ 5,585 \\
\$ 0 \\
\$ 2,739\end{array}$ \\
\hline $\begin{array}{l}\text { Subtotal Estimate } \\
\text { Escalation } \\
\text { Management Reserve }\end{array}$ & & & & $\begin{array}{r}\$ 30 \\
\$ 123\end{array}$ & $\begin{array}{r}\$ 742 \\
\$ 3,061\end{array}$ & $\begin{array}{l}\$ 0 \\
\$ 0\end{array}$ & $\begin{array}{l}\$ 0 \\
\$ 0\end{array}$ & $\$ 00$ & $\begin{array}{r}\$ 8,324 \\
\$ 772 \\
\$ 3,183\end{array}$ \\
\hline
\end{tabular}


Project Name: RTC Waste Heat Recovery

Project Location: ATR

Estimate Number:7850

\section{Code Description Contractor}

\section{Maaln Suppiy Installation (Canal Area 20" Elevation)}

MECH
Overhead Metal Piping Installation Supply Piping

作

$$
\begin{aligned}
& \text { Piping Flange Fitting 4", Installation } \\
& \text { Memo: RSMeans } 2211 \text { 13.47 } \\
& \text { 4" - } 90 \text { Degree Elbow Pipe Fitting Installation } \\
& \text { Memo: RSMeans } 221113.47 \\
& \text { 4" - } 45 \text { Degree Elbow Pipe Fitting Installation } \\
& \text { Memo: RSMeans } 221113.47 \\
& \text { 4" - Tee Straight Pipe Fiting MECH Installation } \\
& \text { Memo: RSMeans } 221113.47
\end{aligned}
$$$$
\text { UC. per Ea }
$$$$
3.00 \mathrm{Ea}
$$

$$
\text { U.C. perEa }
$$

$2.00 \quad \mathrm{Ea}$$$
\text { U.C. perEa }
$$

U.C. per Ea

$2.00 \quad \mathrm{Ea}$

Gasket and Bolt Set
Memo: RSMeans 2211 13.47

\section{DETAIL ITEM REPORT}

Client: C. P. Ischay

\begin{tabular}{|c|c|c|c|c|c|c|c|}
\hline $\begin{array}{l}\text { Subtotal } \\
\text { Sales Tax } \\
\text { Markups }\end{array}$ & $49.03 \%$ & $\begin{array}{r}\$ 7,708 \\
\$ 0 \\
\$ 3,779\end{array}$ & $\begin{array}{l}\$ 0 \\
\$ 0 \\
\$ 0\end{array}$ & $\begin{array}{r}\$ 4,520 \\
\$ 271 \\
\$ 2,349\end{array}$ & $\begin{array}{l}\$ 0 \\
\$ 0 \\
\$ 0\end{array}$ & $\begin{array}{l}\$ 0 \\
\$ 0 \\
\$ 0\end{array}$ & $\begin{array}{r}\$ 12,227 \\
\$ 271 \\
\$ 6,128\end{array}$ \\
\hline $\begin{array}{l}\text { Subtotal Estimate } \\
\text { Escalation } \\
\text { Management Reserve }\end{array}$ & & $\begin{array}{l}\$ 1,065 \\
\$ 4,393\end{array}$ & $\begin{array}{l}\$ 0 \\
\$ 0\end{array}$ & $\begin{array}{r}\$ 662 \\
\$ 2,730\end{array}$ & $\begin{array}{l}\$ 0 \\
\$ 0\end{array}$ & $\begin{array}{l}\$ 0 \\
\$ 0\end{array}$ & $\begin{array}{r}\$ 18,626 \\
\$ 1,727 \\
\$ 7,124\end{array}$ \\
\hline
\end{tabular}

Prepared By: A. W. Miller/S. N. Wasle

Estimate Type: Class-5

Equipment Material Subcontractor Other TOTAL

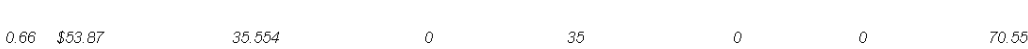


Project Name: RTC Waste Heat Recovery

Project Location: ATR

Estimate Number: 7850

\section{Code Description Contractor}

MNain Retum Installation (Canal Area 20" Elevation)

\section{DETAIL ITEM REPORT}

Client: C.P.Ischay

Prepared By: A. W. Miller/S. N. Wasley

Estimate Type: Class-5

Qty UOM Hrs Resource Labor Equipment Material Subcontractor Other TOTAL

(n)

U.C. per Linit 100.00 Lnft

$\begin{aligned} 0.66 & \$ 53.87 \\ 66 & \text { CNPIPE }\end{aligned}$

35.554
$\$ 3,555$

$\$ 0$

35
$\$ 3,500$

35

$\$ 0$

$\$ 7,554$

Meme

Piping Flange Fitting 4", Installation
Memo: RSMeans 2211 13.47

UC. per Ea

$3.00 \mathrm{Ea}$

$\begin{aligned} 2.6 & \$ 53.87 \\ 8 & \text { CNPIPE }\end{aligned}$

$\$ 40.063$
$\$ 420$

$\$ 0 \quad \$ 33$

MECH
4" - 90 Degree Elbow Pipe Fitting Installation
Memo: RSMeans 221113.47

U.C. per Ea

$\begin{array}{rrrr}2.00 \mathrm{Ea} & 4.5 & 553.87 \\ & 9 & \mathrm{CNPIPE}\end{array}$

242.415
$\$ 485$

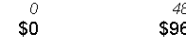

$\$ 0$

0

\begin{tabular}{|c|c|c|c|c|c|c|c|c|c|c|}
\hline $\begin{array}{l}\text { MECH } \\
\text { 4" - 45 Degree Elbow Pipe Fitting Installation } \\
\text { Memo: RSMeans } 221113.47\end{array}$ & UC. perEa & 4.00 & Ea & $\begin{array}{ll}4.5 & 553.87 \\
18 & \text { CNPIPE }\end{array}$ & $\begin{array}{r}242.415 \\
5970\end{array}$ & $\$ 0$ & $\begin{array}{r}49.5 \\
\$ 198\end{array}$ & \$o & so & $\begin{array}{l}291.915 \\
\$ 1,168\end{array}$ \\
\hline $\begin{array}{l}\text { MECH } \\
\text { 4" - Tee Straight Pipe Fiting Installation } \\
\text { Memo: RSMeans } 221113.47\end{array}$ & Uc. perEa & 2.00 & Ea & $\begin{array}{ll}7.5 & 553.87 \\
15 & \text { CNPIPE }\end{array}$ & $\begin{array}{r}404.025 \\
\$ 808\end{array}$ & $\$ 0$ & $\begin{array}{l}178 \\
\$ 236\end{array}$ & $\$ 0$ & $\$ 0$ & $\begin{array}{l}522.025 \\
\$ 1,044\end{array}$ \\
\hline $\begin{array}{l}\text { Gasket and Bolt Set } \\
\text { Memo: RSMeans } 221113.47\end{array}$ & Uc. perEa & 11.00 & Ea & $\begin{aligned} 2.49 & \$ 53.67 \\
27 & \text { CNPIPE }\end{aligned}$ & $\begin{array}{l}133.597 \\
\$ 1,470\end{array}$ & \$o & $\begin{array}{r}35.5 \\
\$ 391\end{array}$ & $\begin{array}{r}0 \\
\$ 0\end{array}$ & \$o & $\begin{array}{l}169.097 \\
\$ 1,860\end{array}$ \\
\hline $\begin{array}{l}\text { Subtotal Estimate } \\
\text { Escalation } \\
\text { Management Reserve }\end{array}$ & & & & & $\begin{array}{l}\$ 1,065 \\
\$ 4,393\end{array}$ & $\$ 00$ & $\begin{array}{r}\$ 662 \\
\$ 2,730\end{array}$ & $\begin{array}{l}\$ 0 \\
\$ 0\end{array}$ & $\begin{array}{l}\$ 0 \\
\$ 0\end{array}$ & $\begin{array}{r}\$ 18,626 \\
\$ 1,727 \\
\$ 7,124\end{array}$ \\
\hline
\end{tabular}

5/11/2012 11:08:1 
Project Name: RTC Waste Heat Recovery

Project Location: ATR

\section{Code Description Contractor}

MMain Suppiv Insulation Installation (Canal Area 20' Elevation)

Memo: RSMeans 220719.30

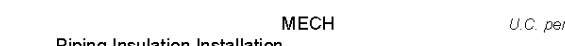

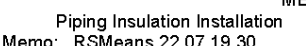

MECH

4.c. per Lnit

9.992

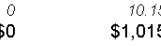

Client:

C.P.Ischay

A.W. Miller/S. N. Wasley

Qty UOM Hrs Resource Labor Equipment Material subcontractor Other TOTAL

\section{Main Retum Insulation Installation (Canal Area $20^{\circ}$ Elevation}

Memo: RSMeans 220719.10$$
\text { MECH }
$$

$\mathrm{MECH}$

uc. per Lint

100.00 Lnft
$\$ 1,993$

0.37 .55887
37
CNPPIPE
$\$ 0$

10,15
$\$ 1,015$
$\$ 0$

$\$$

30.082
$\$ 3,008$ 
Project Name: RTC Waste Heat Recovery

Project Location: ATR

Estimate Number: 7850

\section{Code Description Contractor}

MMain Retum insulation installation (Canal Area 20 Elevation)

Memo: $\quad$ RSMeans 220719.10

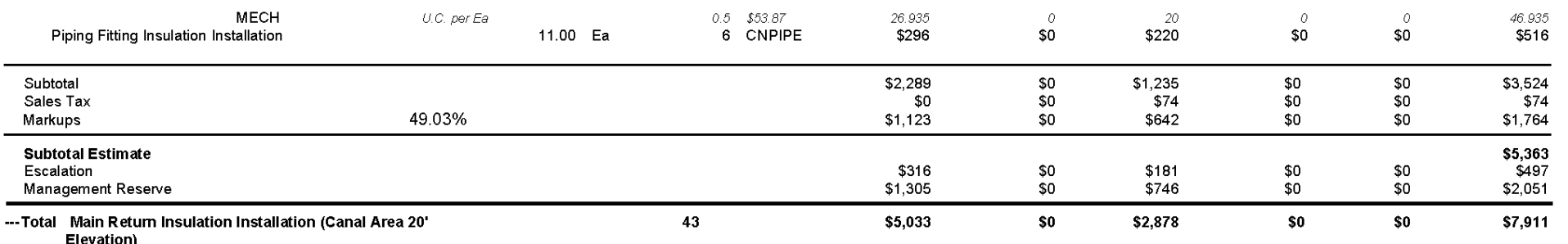

\section{Overhead Working Equipment}

Memo: This is a monthly cost for two snorkel lifts obtained from the Rental Rate Blue Book for Construction Equipment

\begin{tabular}{|c|c|c|c|c|c|c|c|c|c|}
\hline $\begin{array}{l}\text { MECH } \\
\text { Snorkel Lit (2 Each To Spread The Load Of The } \\
\text { Metal Piping) }\end{array}$ & U.C. per Month & 1.00 Month & $\begin{array}{ll}4 & \$ 53.87 \\
4 & \text { CNPIPE }\end{array}$ & $\begin{array}{r}215.46 \\
\$ 215\end{array}$ & $\begin{array}{r}5370 \\
\$ 5,370\end{array}$ & $\$$ & $\begin{array}{l}0 \\
\$ 0\end{array}$ & \$o & $\begin{array}{l}5585,48 \\
\$ 5,585\end{array}$ \\
\hline $\begin{array}{l}\text { Subtotal } \\
\text { Sales Tax } \\
\text { Markups }\end{array}$ & $49.03 \%$ & & & $\begin{array}{r}\$ 215 \\
\$ 0 \\
\$ 106\end{array}$ & $\begin{array}{r}\$ 5,370 \\
\$ 0 \\
\$ 2,633\end{array}$ & $\begin{array}{l}\$ 0 \\
\$ 0 \\
\$ 0\end{array}$ & $\begin{array}{l}\$ 0 \\
\$ 0 \\
\$ 0\end{array}$ & $\begin{array}{l}\$ 0 \\
\$ 0 \\
\$ 0\end{array}$ & $\begin{array}{r}\$ 5,585 \\
\$ 0 \\
\$ 2,739\end{array}$ \\
\hline $\begin{array}{l}\text { Subtotal Estimate } \\
\text { Escalation } \\
\text { Management Reserve }\end{array}$ & & & & $\begin{array}{r}\$ 30 \\
\$ 123\end{array}$ & $\begin{array}{r}\$ 742 \\
\$ 3,061\end{array}$ & $\begin{array}{l}\$ 0 \\
\$ 0\end{array}$ & $\begin{array}{l}\$ 0 \\
\$ 0\end{array}$ & $\begin{array}{l}\$ 0 \\
\text { \$0 }\end{array}$ & $\begin{array}{r}\$ 8,324 \\
\$ 772 \\
\$ 3,183\end{array}$ \\
\hline Total Overhead Working Equipment & & & & $\$ 474$ & $\$ 11,805$ & \$o & \$o & so & $\$ 12,279$ \\
\hline
\end{tabular}

11:08:15
DETAIL ITEM REPORT

Client: $\quad$ C.P. Ischay

Prepared By: A. W. Miller/S. N. Wasley

\section{Qty UOM Hrs Resource Labor Equipment Material Subcontractor Other TOTAL}

Total Overhead Working Equipment 
Project Name: RTC Waste Heat Recovery

Project Location: ATR

Estimate Number: 7850

\section{Code Description Contractor \\ 5.1.2.2.9 PIpIn}

nemo:

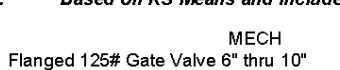

Flanged 125\# Gate Valve $6 " \mathrm{MECH}$

Flanged 150\# Check Valve $10^{\prime \prime}$

$\mathrm{MECH}$
Flanged 150 Actuated Control Valves 4"

Pressure Relief Valves 2"-4"

Slip-on Flanges 150\# 4" thru 6"

MECH
Slip-on Flanges 150\# 8" thru 10"

$\mathrm{MECH}$

Gasket and Bolt Set 4" thru $6^{\prime \prime}$

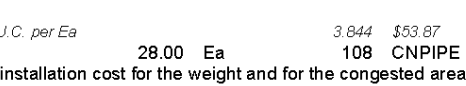

$\begin{array}{rrrr} & 14.89 & \$ 53.87 \\ 00 & \text { Ea } & 119 & \text { CNPIPE }\end{array}$

per E

$2.00 \mathrm{Ea}$

$\begin{aligned} 16.895 & \$ 53.87 \\ 34 & \text { CNPIPE }\end{aligned}$

$\begin{array}{ll}9.3 & \$ 53.87 \\ 93 & \text { CNPIPE }\end{array}$

10.00

$\begin{aligned} 7.75 & \$ 53.87 \\ 31 & \text { CNPIPE }\end{aligned}$

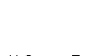

$4.00 \mathrm{Ea}$

(a)

$\begin{array}{ll}4.96 & 553.87 \\ 139 & \text { CNPIPE }\end{array}$

$28.00 \mathrm{Ea}$

$\begin{array}{rl}9.3 & 553.87 \\ 149 & \text { CNPIPE }\end{array}$

U. per Ea $\quad 16.00$

$$
\begin{gathered}
M E C H \\
\text { Gasket and Bolt Set } 8 \text { " thru } 10 "
\end{gathered}
$$

$$
\text { U.C. per Ea }
$$

$16.00 \mathrm{Ea}$

$\begin{array}{ll}3.844 & \$ 53.87 \\ 62 & \text { CNPIPE }\end{array}$

207.076
$\$ 3,313$

u. per Ea

$4.00 \quad \mathrm{Ea}$

$\begin{array}{ll}9.3 & 553.87 \\ 37 & \text { CNPIPE }\end{array}$

500.99
$\$ 2,004$

HVAC Control System ELECT Memo: Based on estimator judgment.

Heating Coil HVS-1 (size $\left.10^{\mathrm{MECH}} \times 12^{\prime}\right)$

$M E C H$
Heating Coil HVS-2 (size $\left.10^{\prime} \times 12^{\prime}\right)$

4.0. per Ea

$1.00 \mathrm{Ea}$

$\begin{array}{ll}200 & 550.06 \\ 200 & \text { CNELEC }\end{array}$

10012
$\$ 10,012$

U. persF $120.00 \mathrm{SF}$

$\begin{array}{ll}0.31 & 553.67 \\ 37 & \text { CNPIPE }\end{array}$

UC. per SF $\quad 120.00 \quad$ SF

$\begin{array}{ll}0.31 & 553.87 \\ 37 & \text { CNPIPE }\end{array}$

Cost Estimating

BEA

11:08:15 r congested areas. RSMeans)

Flex Couplings 6" thru 10" MECH

Client: C. P. Ischay

Prepared By: A. W. Miller/S. N. Wasley

Estimate Type: Class-5

Equipment Material Subcontractor Other TOTAL

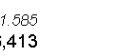

910.135

$\begin{array}{rrrr}2925 & 0 & 0 & 3726.585 \\ \$ 23,400 & \$ 0 & \$ 0 & \$ 29,813\end{array}$

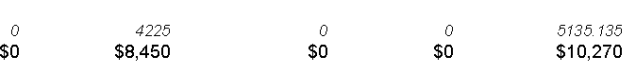

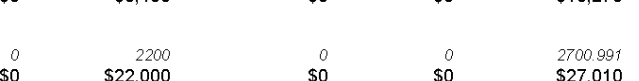

$\begin{array}{rrrr}\$ 0 & \$ 0 & \$ 0 & \\ 0 & 4300 & 0 & 0\end{array}$

$\begin{array}{llll}\$ 17,200 & \$ 0 & \$ 0 & \$ 7177,493 \\ & \$ 18,870\end{array}$

$\begin{array}{rrrrr}\$ 0 & 58 & 0 & 0 & 325,195 \\ \$ 0 & \$ 1,624 & \$ 0 & \$ 0 & \$ 9,105\end{array}$

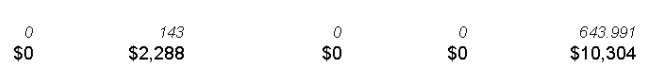

$\begin{array}{rrrrr}0 & 35.5 & 0 & 0 & 242.576 \\ \$ 0 & \$ 994 & \$ 0 & \$ 0 & \$ 6,792\end{array}$

$\begin{array}{rrrrr}0 & 35.5 & 0 & 0 & 242.576 \\ \$ 0 & \$ 568 & \$ 0 & \$ 0 & \$ 3,881\end{array}$

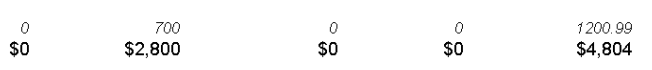

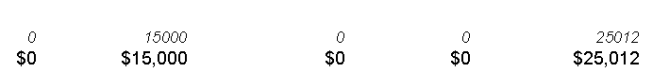

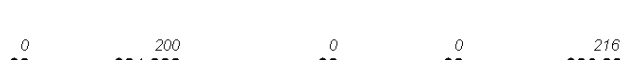

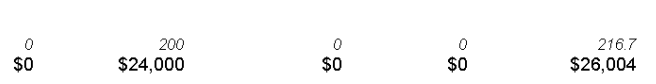


Project Name: RTC Waste Heat Recovery

Project Location: ATR

Pste 7850

Code Description contractor

5.1.2.2.9 PIpinq, Valve, Colls, \& Control System

Memo:

\begin{tabular}{|c|c|c|c|c|c|}
\hline $\begin{array}{r}M E C H \\
\left.\text { Heating Coil HVS-3 (size } 10^{\prime} \times 12^{\prime}\right)\end{array}$ & U. persf & 120.00 & $S F$ & $\begin{array}{r}0.31 \\
37\end{array}$ & $\begin{array}{l}\$ 53.87 \\
\text { CNPIPE }\end{array}$ \\
\hline $\begin{array}{r}M E C H \\
\left.\text { Heating Coil HVS-4 (size } 10^{\prime} \times 12^{\prime}\right)\end{array}$ & U.C. perSF & 120.00 & SF & $\begin{array}{r}0.31 \\
37\end{array}$ & $\begin{array}{l}\$ 53.87 \\
\text { CNPIPE }\end{array}$ \\
\hline $\begin{array}{r}\text { MECH } \\
\left.\text { Heating Coil HVS-5 (size } 10^{\prime} \times 12^{\prime}\right)\end{array}$ & U.C. persF & 120.00 & SF & $\begin{array}{r}0.31 \\
37\end{array}$ & $\begin{array}{l}\$ 53.87 \\
\text { CNPIPE }\end{array}$ \\
\hline
\end{tabular}

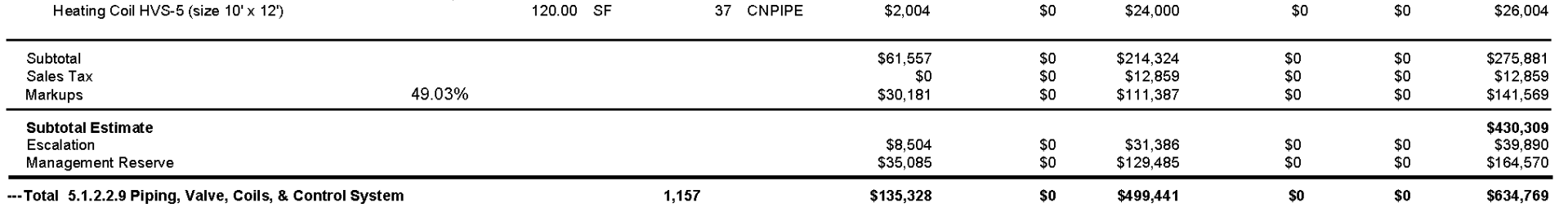

Building Piping Vacuum Excavations

Memo: Due to the number of utilifies located on the north side of Building TRA-670, 100\% of the excavations will be performed by a vacuum excavator.

\begin{tabular}{|c|c|c|c|c|c|c|c|c|c|}
\hline $\begin{array}{l}\text { EARTHW } \\
\text { Vacuum Excavations For The Heat Recovery Water } \\
\text { Lines }\left(80^{\circ} \times 7^{\prime} \times 7^{\prime}\right)\end{array}$ & uce percyd & $150.00 \mathrm{Cyd}$ & 0 & \$o & $\$ 0$ & $\$ 0$ & $\begin{array}{r}357 \\
\$ 53,550\end{array}$ & $\$ 0$ & $\begin{array}{r}357 \\
\$ 53,550\end{array}$ \\
\hline $\begin{array}{l}\text { Subtotal } \\
\text { Sales Tax } \\
\text { Markups }\end{array}$ & $49.03 \%$ & & & $\begin{array}{l}\$ 0 \\
\$ 0 \\
\$ 0\end{array}$ & $\begin{array}{l}\$ 0 \\
\$ 0 \\
\$ 0\end{array}$ & $\begin{array}{l}\$ 0 \\
\$ 0 \\
\$ 0\end{array}$ & $\begin{array}{l}\$ 53,550 \\
\$ 0 \\
\$ 26,255\end{array}$ & $\begin{array}{l}\$ 0 \\
\$ 0 \\
\$ 0\end{array}$ & $\begin{array}{r}\$ 53,550 \\
\$ 0 \\
\$ 26,255\end{array}$ \\
\hline $\begin{array}{l}\text { Subtotal Estimate } \\
\text { Escalation } \\
\text { Management Reserve }\end{array}$ & & & & $\begin{array}{l}\$ 0 \\
\$ 0\end{array}$ & $\begin{array}{l}\$ 0 \\
\$ 0\end{array}$ & $\begin{array}{l}\$ 0 \\
\$ 0\end{array}$ & $\begin{array}{r}\$ 7,398 \\
\$ 30,521\end{array}$ & $\begin{array}{l}\$ 0 \\
\$ 0\end{array}$ & $\begin{array}{r}\$ 79,805 \\
\$ 7,398 \\
\$ 30,521\end{array}$ \\
\hline
\end{tabular}


Project Name: RTC Waste Heat Recovery

Project Location: ATR

Hstimate Number:7850

\section{Code Description Contractor}

Pump House Bullaing Foundation Excavation

Due to the number of utilitites located on the north side of Building TRA-670, $100 \%$ of the excavations will be performed by a vacuum excavator.

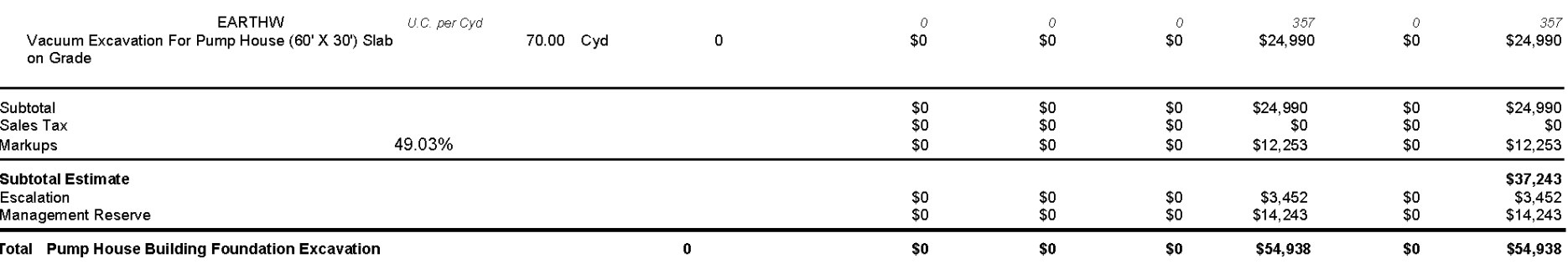

\section{Backfill Excavations}

Memo: RSMeans $312323.13(0100)$

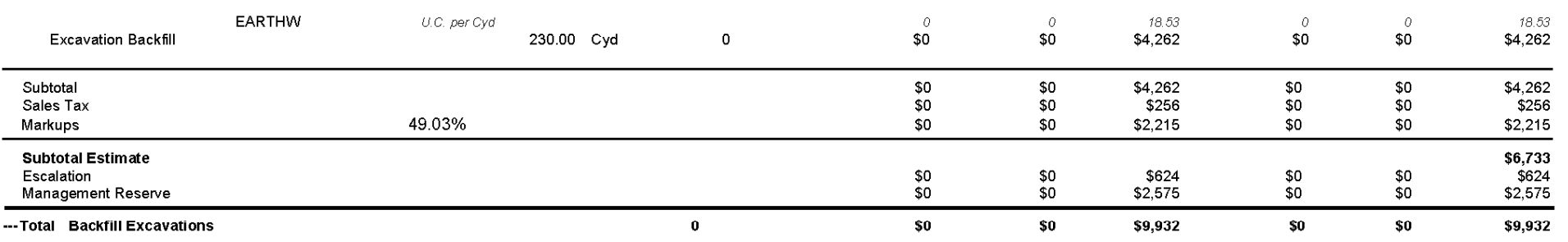

Building Concrete Footings

Heat Exchanger Footing
Memo: RSMeans 033053.40 This is only for the concrete and rebar installations.

BEA

11:08:15

Cost Estimating

Client: $\quad$ C. P. Ischay

Prepared By: A.W. Miller/S. N. Wasley

Subcontractor Other TOTAL 
Project Name: RTC Waste Heat Recovery

Project Location: ATR

Code Description

Bullaung Concrete Fooun

\begin{tabular}{|c|c|c|c|c|c|c|c|}
\hline Subtotal & & $\$ 0$ & $\$ 0$ & $\$ 0$ & $\$ 4,983$ & $\$ 0$ & $\$ 4,983$ \\
\hline $\begin{array}{l}\text { Sales Tax } \\
\text { Markups }\end{array}$ & $49.03 \%$ & $\$ 0$ & $\$ 0$ & $\begin{array}{l}\$ 0 \\
\$ 0\end{array}$ & $\$ \$ 0$ & 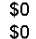 & $\begin{array}{r}\$ 0 \\
\$ 2,443 \\
\end{array}$ \\
\hline Subtotal Estimate & & & & & & & $\$ 7,426$ \\
\hline $\begin{array}{l}\text { Escalation } \\
\text { Management Reserve }\end{array}$ & & $\begin{array}{l}\$ 0 \\
\$ 0\end{array}-10$ & $\begin{array}{l}\$ 0 \\
\$ 0\end{array}-10$ & $\begin{array}{l}\$ 0 \\
\$ 0\end{array}$ & $\begin{array}{l}\$ 2,840 \\
\$ 640\end{array}$ & $\begin{array}{l}\$ 0 \\
\$ 0\end{array}-10$ & $\begin{array}{r}\$ \$, 888 \\
\$ 2,840\end{array}$ \\
\hline
\end{tabular}

\section{Building Concrete Foundation}

Memo: RSMeans 033053.40 This is only for the concrete and rebar installations

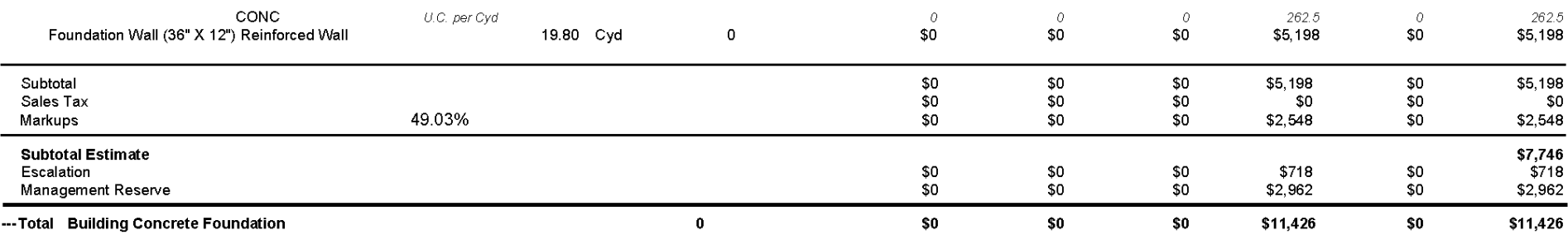

Bullana concrete slap

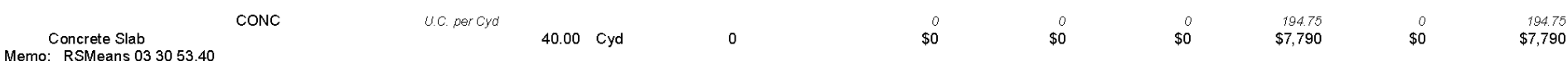

5/11/2012 11:08:15

\section{DETAIL ITEM REPORT}

Client: C.P.Ischay

Prepared By: A. W. Miller/S. N. Wasley

Subcontractor Other TOTAL

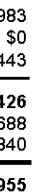

(n)

Memo: RSMeans 033053.40

(2)

(n)


Project Name: RTC Waste Heat Recovery

Project Location: ATR

Estimate Number: 7850

Code Description

Bullalng Concrete SIa

\begin{tabular}{|c|c|c|c|c|c|c|c|}
\hline $\begin{array}{l}\text { Subtotal } \\
\text { Sales Tax } \\
\text { Markups }\end{array}$ & $49.03 \%$ & $\begin{array}{l}\$ 0 \\
\$ 0 \\
\$ 0 \\
\end{array}$ & $\begin{array}{l}\$ 0 \\
\$ 0 \\
\$ 0 \\
\end{array}$ & $\begin{array}{l}\$ 0 \\
\$ 0 \\
\$ 0 \\
\end{array}$ & $\begin{array}{l}\$ 7,790 \\
\$ 0 \\
\$ 3,819 \\
\end{array}$ & $\begin{array}{l}\$ 0 \\
\$ 0 \\
\$ 0\end{array}$ & $\begin{array}{r}\$ 7,790 \\
\$ 0 \\
\$ 3,819 \\
\end{array}$ \\
\hline $\begin{array}{l}\text { Subtotal Estimate } \\
\text { Escalation } \\
\text { Management Reserve }\end{array}$ & & $\begin{array}{l}\$ 0 \\
\$ 0\end{array}$ & $\begin{array}{l}\$ 0 \\
\$ 0\end{array}$ & $\begin{array}{l}\$ 0 \\
\$ 0\end{array}$ & $\begin{array}{l}\$ 1,076 \\
\$ 4,440\end{array}$ & $\begin{array}{l}\$ 0 \\
\$ 0\end{array}$ & $\begin{array}{r}\$ 11,609 \\
\$ 1,076 \\
\$ 4,440\end{array}$ \\
\hline
\end{tabular}

\subsubsection{Heat Exchanger Building Exterior}

\begin{tabular}{|c|c|c|c|c|c|c|c|c|c|c|}
\hline $\begin{array}{l}\text { GEN } \\
\text { Heat Exchanger Building }\left(60^{\prime} \times 30^{\prime} \times 20^{\prime}\right) \text { Surface } \\
\text { Area } \\
\text { mo: RSMeans B2010 } 143\end{array}$ & Uc. per Sqft & $4,800.00$ & Sqft & 0 & $\$ 0$ & $\$ 0$ & $\$ 0$ & $\begin{array}{r}10.18 \\
\$ 48,864\end{array}$ & $\$ 0$ & $\begin{array}{r}10,18 \\
\$ 48,864\end{array}$ \\
\hline $\begin{array}{l}\text { ROOF } \\
\text { Roofing Materials and Labor } \\
\text { mo: RSMeans B3010 } 120\end{array}$ & uc. per sqitt & $1,800.00$ & Sqft & 0 & \$o & \$0 & \$o & $\begin{array}{r}3.25 \\
\$ 5,850\end{array}$ & \$o & $\begin{array}{r}3.25 \\
\$ 5,850\end{array}$ \\
\hline $\begin{array}{l}\text { Subtotal } \\
\text { Sales Tax } \\
\text { Markups }\end{array}$ & $37.18 \%$ & & & & $\begin{array}{l}\$ 0 \\
\$ 0 \\
\$ 0\end{array}$ & $\begin{array}{l}\$ 0 \\
\$ 0 \\
\$ 0\end{array}$ & $\begin{array}{l}\$ 0 \\
\$ 0 \\
\$ 0\end{array}$ & $\begin{array}{r}\$ 54,714 \\
\$ 0 \\
\$ 20,343\end{array}$ & $\begin{array}{l}\$ 0 \\
\$ 0 \\
\$ 0\end{array}$ & $\begin{array}{r}\$ 54,714 \\
\$ 0 \\
\$ 20,343\end{array}$ \\
\hline Total 3.1.3.3 Heat Exchanger Bullang Extenor & & & & & $\$ 0$ & $\$ 0$ & $\$ 0$ & $\$ 110, r 20$ & \$0 & $\$ 110, r 20$ \\
\hline
\end{tabular}

5.1.3.4 Heat Exchanger Building Interior

Interior Wall and Door Allowance

11:08:15

\section{DETAIL ITEM REPORT}

Client: C.P.Ischay

Prepared By. A.W.Miller/S. N. Wasley

Estimate Type: Class-5

Subcontractor Other TOTAL

\section{\$1 \\ $\begin{array}{r}1,609 \\ 4,440 \\ \hline\end{array}$}

-Total Building Concrete Slab

so


Project Name: RTC Waste Heat Recovery

Project Location: ATR

Estimate Number: 7850

Code Description $\quad$ contractor

5.1.3.4 Heat Exchanger Bullaing interior

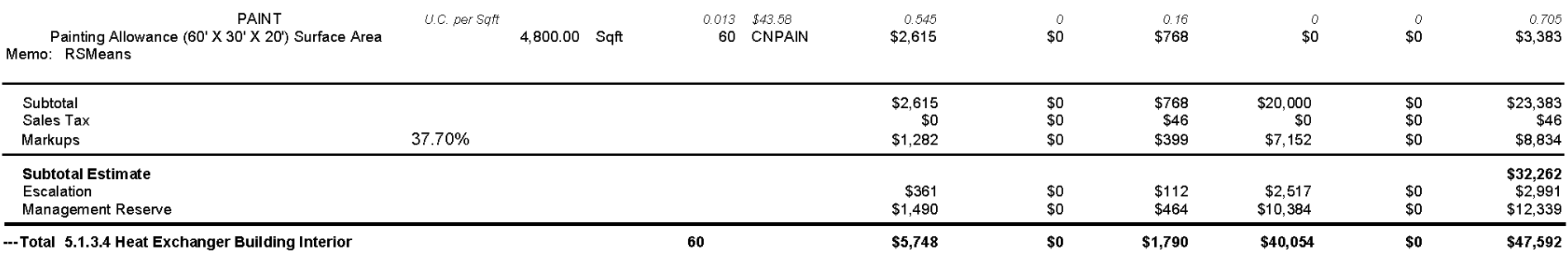

5.1.3.5 Heat Exchanger Building HVAC

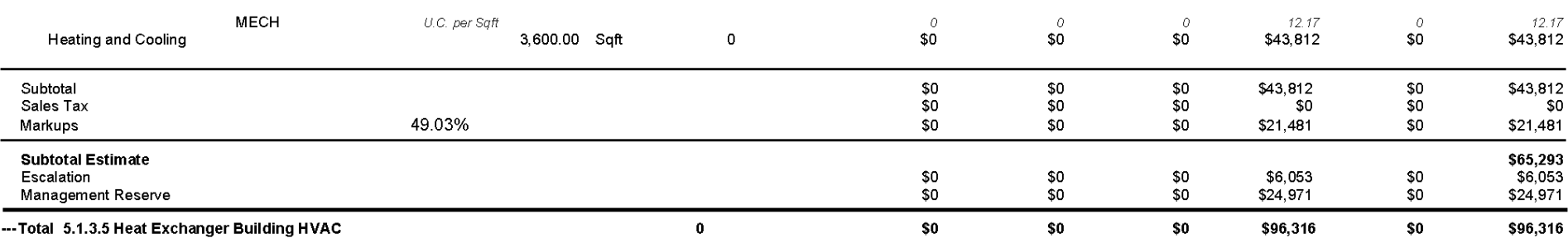

\section{Electrical Building Service Connection}

Memo: This is based on a 1000 amp 3 phase electrical service obtained from RSMeans. D5010
Service Connection
ELECT
U.C. per Allow
1.00 Allow $\quad 0$

11:08:15

DETAIL ITEM REPORT

Client: $\quad$ C. P. Ischay

Prepared By: A.W. Miller/S. N. Wasley

Estimate Type: Class-5

Qty UOM Hrs Resource Labor Equipment Material Subcontractor Other TOTAL

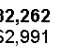
47,592

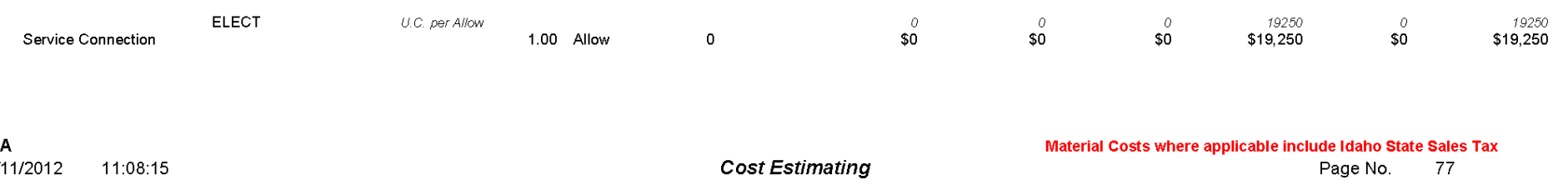


Project Name: RTC Waste Heat Recovery

Project Location: ATR

Code Description $\quad$ contractor EElectrical Bullaing Service Connection

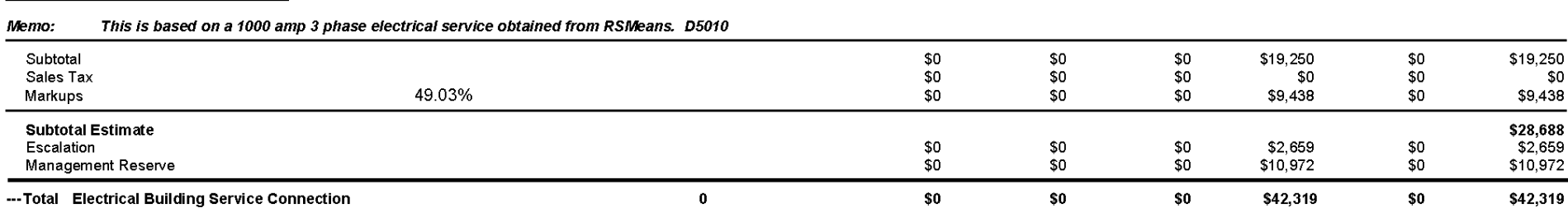

General Electrical Lighting and Outlets

Memo: Based on the square footage of the proposed building. RSMeans 5020

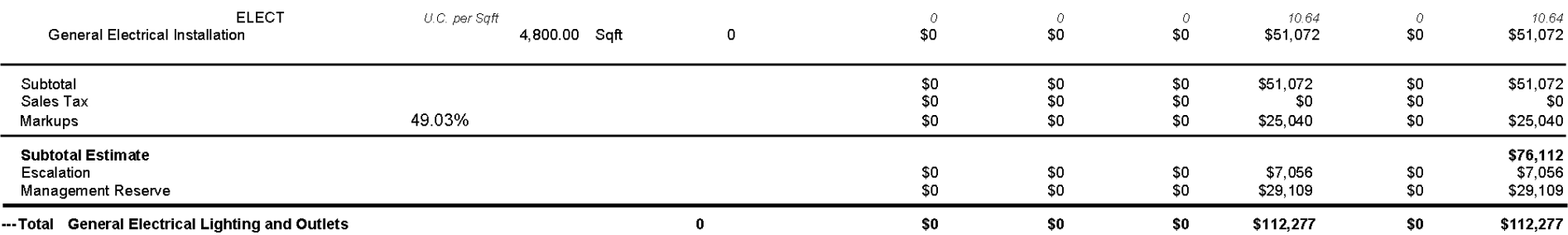

\section{Pump House Communication and Fire Alarm System}

Memo: Based on the square footage of the building. RSMeans

$\begin{array}{ccc}\text { ELECT } & \text { U.C. per Allow } 1.00 \text { Allow }\end{array}$

Memo: RSMeans D5030

$\$ 0$

$\begin{array}{r}13950 \\ \$ 0\end{array} \quad \$ 13,950$

$\$ 0$

Client: C. P. Ischay

Prepared By: A. W. Miller/S. N. Wasley

Subcontractor Other TOTAL $\begin{array}{r}\$ 0 \\ 438 \\ \hline\end{array}$ $\$ 28,688$
$\$ 2,659$
10,972 $\$ 10,972$ 
Project Name: RTC Waste Heat Recovery

Project Location: ATR

Code Description

Pump House Communication and Fire Alarm System

Memo: Based on the square footage of the building. RSMeans

\begin{tabular}{|c|c|c|c|c|c|c|c|}
\hline $\begin{array}{l}\text { Subtotal } \\
\text { Sales Tax } \\
\text { Markups }\end{array}$ & $49.03 \%$ & $\begin{array}{l}\$ 0 \\
\$ 0 \\
\$ 0\end{array}$ & $\begin{array}{l}\$ 0 \\
\$ 0 \\
\$ 0\end{array}$ & $\begin{array}{l}\$ 0 \\
\$ 0 \\
\$ 0\end{array}$ & $\begin{array}{r}\$ 13,950 \\
\$ 0 \\
\$ 6,840\end{array}$ & $\begin{array}{l}\$ 0 \\
\$ 0 \\
\$ 0\end{array}$ & $\begin{array}{r}\$ 13,950 \\
\$ 0 \\
\$ 6,840\end{array}$ \\
\hline $\begin{array}{l}\text { Subtotal Estimate } \\
\text { Escalation } \\
\text { Management Reserve }\end{array}$ & & $\begin{array}{l}\$ 0 \\
\$ 0\end{array}$ & $\begin{array}{l}\$ 0 \\
\$ 0\end{array}$ & $\begin{array}{l}\$ 0 \\
\$ 0\end{array}$ & $\begin{array}{l}\$ 1,927 \\
\$ 7,951\end{array}$ & $\begin{array}{l}\$ 0 \\
\$ 0\end{array}$ & $\begin{array}{r}\$ 20,790 \\
\$ 1,927 \\
\$ 7,951\end{array}$ \\
\hline
\end{tabular}

Pump \& Control Connections

\begin{tabular}{|c|c|c|c|c|c|c|c|c|c|c|c|}
\hline $\begin{array}{l}\text { ELECT } \\
\text { Pump Connection Allowance } \\
\text { Memo: Based on estimator judgment. }\end{array}$ & UC. perEa & 2.00 & & $\begin{array}{l}20 \\
40\end{array}$ & $\begin{array}{l}\$ 50.06 \\
\text { CNELEC }\end{array}$ & $\begin{array}{r}1001.2 \\
\$ 2,002\end{array}$ & $\$$ & $\begin{array}{r}20000 \\
\$ 40,000\end{array}$ & $\begin{array}{l}0 \\
\$ 0\end{array}$ & \$o & $\begin{array}{r}21001.2 \\
\$ 42,002\end{array}$ \\
\hline $\begin{array}{l}\text { ELECT } \\
\text { Connect Electrical Control Valves ( } 500 \text { ' per Valve } \\
\times 10 \text { Each) }\end{array}$ & U.c. perEa & $5,000.00$ & Ea & $\begin{array}{l}0.12 \\
600\end{array}$ & $\begin{array}{l}\$ 50.06 \\
\text { CNELEC }\end{array}$ & $\begin{array}{r}6.007 \\
\$ 30,036\end{array}$ & $\$ 0$ & $\begin{array}{r}3.5 \\
\$ 17,500\end{array}$ & $\begin{array}{l}0 \\
\$ 0\end{array}$ & $\begin{array}{r}0 \\
\$ 0\end{array}$ & $\begin{array}{r}9.507 \\
\$ 47,536\end{array}$ \\
\hline $\begin{array}{l}\text { Subtotal } \\
\text { Sales Tax } \\
\text { Markups }\end{array}$ & $49.03 \%$ & & & & & $\begin{array}{l}\$ 32,038 \\
\$ 0 \\
\$ 15,708\end{array}$ & $\begin{array}{l}\$ 0 \\
\$ 0 \\
\$ 0\end{array}$ & $\begin{array}{r}\$ 57,500 \\
\$ 3,450 \\
\$ 29,884\end{array}$ & $\begin{array}{l}\$ 0 \\
\$ 0 \\
\$ 0\end{array}$ & $\begin{array}{l}\$ 0 \\
\$ 0 \\
\$ 0\end{array}$ & $\begin{array}{r}\$ 89,538 \\
\$ 3,450 \\
\$ 45,592\end{array}$ \\
\hline $\begin{array}{l}\text { Subtotal Estimate } \\
\text { Escalation } \\
\text { Management Reserve }\end{array}$ & & & & & & $\begin{array}{r}\$ 4,426 \\
\$ 18,260\end{array}$ & $\begin{array}{l}\$ 0 \\
\$ 0\end{array}$ & $\begin{array}{r}\$ 8,420 \\
\$ 34,739\end{array}$ & $\begin{array}{l}\$ 0 \\
\$ 0\end{array}$ & $\begin{array}{l}\$ 0 \\
\$ 0\end{array}$ & $\begin{array}{r}\$ 138,580 \\
\$ 12,846 \\
\$ 52,999\end{array}$ \\
\hline -Total Pump \& Control Connectıons & & & & & & $\$ / 0,433$ & \$o & 5135,993 & $\$ 0$ & $\$ 0$ & $\$ 204,426$ \\
\hline
\end{tabular}

Liahtning Protection

ELEC

Lightning Protection Allowance

1.00 Allow 0

BEA

11:08:15

Client: C.P.Ischay

Prepared By: A.W. Miller/S. N. Wasley

Estimate Type: Class-5

Qty UOM Hrs Resource Labor Equipment Material Subcontractor Other TOTAL \begin{tabular}{l}
950 \\
50 \\
840 \\
\hline
\end{tabular}

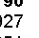

$\$ 7,951$ 
Project Name: RTC Waste Heat Recovery

Project Location: ATR

Code Description LIantning Protection

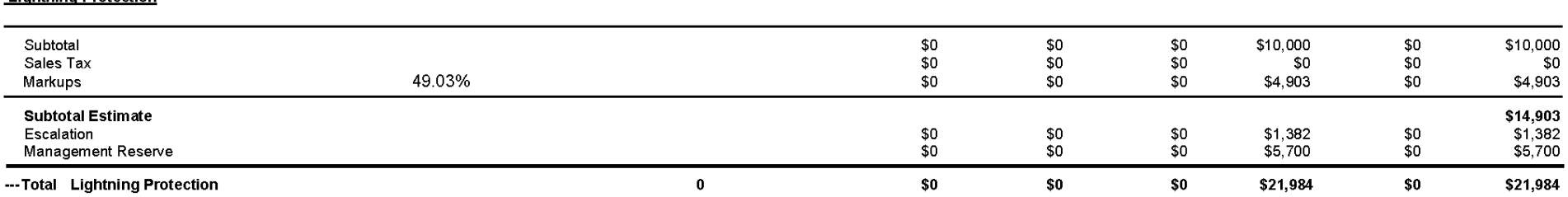

\subsubsection{Building Fire Suppression Svstem}

\begin{tabular}{|c|c|c|c|c|c|c|c|c|c|c|c|}
\hline \multirow{2}{*}{$\begin{array}{l}\text { Memo: } \quad \text { Pricing was provi } \\
\text { Fire Suppression Riser }\end{array}$} & \multirow{2}{*}{$\begin{array}{l}\text { ded by a } \\
\text { FIRE }\end{array}$} & \multirow[b]{2}{*}{ U.C. per Allow } & \multirow[b]{2}{*}{1.00} & \multirow[b]{2}{*}{ Allow } & \multirow[b]{2}{*}{0} & \multirow[b]{2}{*}{$\begin{array}{r}0 \\
\$ 0\end{array}$} & \multirow[b]{2}{*}{ \$o } & \multirow[b]{2}{*}{$\begin{array}{r}0 \\
\$ 0\end{array}$} & \multirow[b]{2}{*}{$\begin{array}{r}4726 \\
\$ 4,726\end{array}$} & \multirow[b]{2}{*}{ \$o } & \multirow[b]{2}{*}{$\begin{array}{r}4726 \\
\$ 4,726\end{array}$} \\
\hline & & & & & & & & & & & \\
\hline Fire Suppression System & FIRE & U.c. per Saft & $4,800.00$ & Sqft & 0 & $\$ 0$ & $\$$ & $\begin{array}{r}0 \\
\$ 0\end{array}$ & $\begin{array}{r}7,23 \\
\$ 34,704\end{array}$ & $\$ 0$ & $\begin{array}{r}7.23 \\
\$ 34,704\end{array}$ \\
\hline $\begin{array}{l}\text { Subtotal } \\
\text { Sales Tax } \\
\text { Markups }\end{array}$ & & $49.03 \%$ & & & & $\begin{array}{l}\$ 0 \\
\$ 0 \\
\$ 0\end{array}$ & $\begin{array}{l}\$ 0 \\
\$ 0 \\
\$ 0\end{array}$ & $\begin{array}{l}\$ 0 \\
\$ 0 \\
\$ 0\end{array}$ & $\begin{array}{r}\$ 39,430 \\
\$ 0 \\
\$ 19,332\end{array}$ & $\begin{array}{l}\$ 0 \\
\$ 0 \\
\$ 0\end{array}$ & $\begin{array}{r}\$ 39,430 \\
\$ 0 \\
\$ 19,332\end{array}$ \\
\hline $\begin{array}{l}\text { Subtotal Estimate } \\
\text { Escalation } \\
\text { Management Reserve }\end{array}$ & & & & & & $\begin{array}{l}\$ 0 \\
\$ 0\end{array}$ & $\begin{array}{l}\$ 0 \\
\$ 0\end{array}$ & $\begin{array}{l}\$ 0 \\
\$ 0\end{array}$ & $\begin{array}{r}\$ 5,447 \\
\$ 22,473\end{array}$ & $\begin{array}{l}\$ 0 \\
\$ 0\end{array}$ & $\begin{array}{r}\$ 58,762 \\
\$ 5,447 \\
\$ 22,473\end{array}$ \\
\hline
\end{tabular}

5.1.4 Waste Heat Recovery Me-in

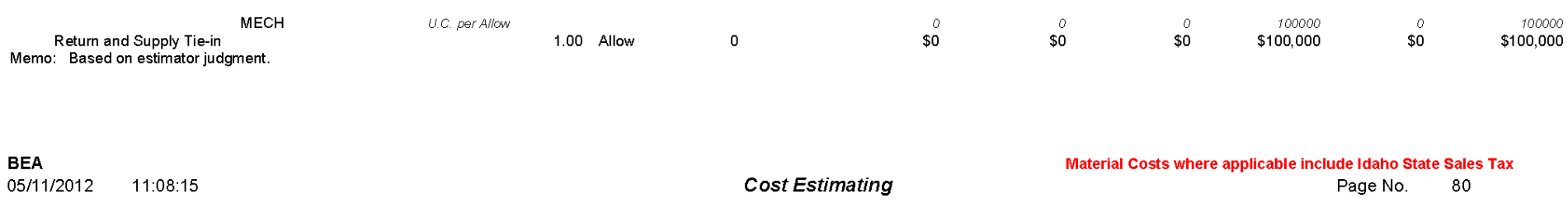


Project Name: RTC Waste Heat Recovery

Project Location: ATR

Estimate Number: 7850

Code Description

5.1.4 Waste Heat Recovery ne-11

\begin{tabular}{|c|c|c|c|c|c|c|c|}
\hline $\begin{array}{l}\text { Subtotal } \\
\text { Sales Tax } \\
\text { Markups }\end{array}$ & $49.03 \%$ & $\begin{array}{l}\$ 0 \\
\$ 0 \\
\$ 0\end{array}$ & $\begin{array}{l}\$ 0 \\
\$ 0 \\
\$ 0\end{array}$ & $\begin{array}{l}\$ 0 \\
\$ 0 \\
\$ 0\end{array}$ & $\begin{array}{r}\$ 100,000 \\
\$ 0 \\
\$ 49,030\end{array}$ & $\begin{array}{l}\$ 0 \\
\$ 0 \\
\$ 0\end{array}$ & $\begin{array}{r}\$ 100,000 \\
\$ 0 \\
\$ 49,030 \\
\end{array}$ \\
\hline $\begin{array}{l}\text { Subtotal Estimate } \\
\text { Escalation } \\
\text { Management Reserve }\end{array}$ & & $\begin{array}{l}\$ 0 \\
\$ 0\end{array}$ & $\begin{array}{l}\$ 0 \\
\$ 0\end{array}$ & $\begin{array}{l}\$ 0 \\
\$ 0\end{array}$ & $\begin{array}{l}\$ 13,815 \\
\$ 56,996\end{array}$ & $\begin{array}{l}\$ 0 \\
\$ 0\end{array}$ & $\begin{array}{r}\mathbf{\$ 1 4 9 , 0 3 0} \\
\$ 13,815 \\
\$ 56,996\end{array}$ \\
\hline
\end{tabular}

\subsubsection{Heat Exchanger}

Memo: RSMeans 235719.13

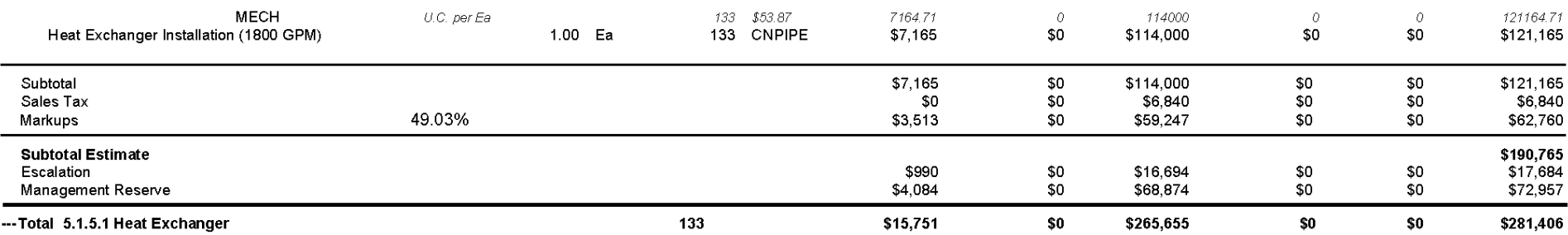

5.1.5.2 Heat Exchanger PIpıng

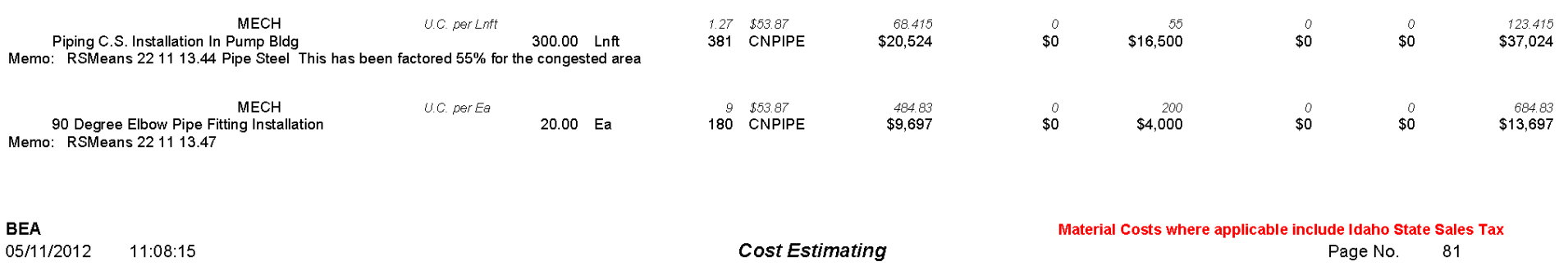

Client: $\quad$ c. P. Ischay

Prepared By: A. W. Miller/S. N. Wasle
Estimate Type: Class-5

Qty UOM Hrs Resource Labor Equipment Material Subcontractor Other TOTAL

sons

9,840 
Project Name: RTC Waste Heat Recovery

Project Location: ATR

\section{Code Description Contractor}

5.1.5.2 Heat Exchanger Plping

MECH

MECH
Flanged 125\# Gate Valve 6" thru 10"

$\begin{aligned} & \text { MECH } \\ & \text { Flanged 150\# Check Valve 10" }\end{aligned}$

$\underset{\sim}{\sim}$
45 Degree Elbow Pipe Fitting Installation

Memo: RSMeans 221113.47

\section{DETAIL ITEM REPORT}

Qty UOM

Hrs Resource Labor

Equipment

Material Subcontractor

Other

TOTAL

\begin{tabular}{|c|c|c|c|c|c|c|c|c|c|c|}
\hline U.C. perEa & 10.00 & $\mathrm{Ea}$ & $\begin{array}{r}9 \\
90\end{array}$ & $\begin{array}{l}\$ 53.87 \\
\text { CNPIPE }\end{array}$ & $\begin{array}{r}484.83 \\
\$ 4,848\end{array}$ & $\$ 0$ & $\begin{array}{r}163 \\
\$ 1,630\end{array}$ & $\begin{array}{l}0 \\
\$ 0\end{array}$ & $\$ 0$ & $\begin{array}{r}647.83 \\
\$ 6,478\end{array}$ \\
\hline UC. perEa & 6.00 & $\mathrm{Ea}$ & $\begin{array}{r}13.44 \\
81\end{array}$ & $\begin{array}{l}\text { \$53.87 } \\
\text { CNPIPE }\end{array}$ & $\begin{array}{l}724.013 \\
\$ 4,344\end{array}$ & \$0 & $\begin{array}{r}355 \\
\$ 2,130\end{array}$ & $\begin{array}{l}0 \\
\$ 0\end{array}$ & $\begin{array}{l}0 \\
\$ 0\end{array}$ & $\begin{array}{r}1079.013 \\
\$ 6,474\end{array}$ \\
\hline U. perEa & 16.00 & Ea & $\begin{array}{r}9.6 \\
154\end{array}$ & $\begin{array}{l}553.87 \\
\text { CNPIPE }\end{array}$ & $\begin{array}{l}517.152 \\
\$ 8,274\end{array}$ & $\$ 0$ & $\begin{array}{r}2925 \\
\$ 46,800\end{array}$ & $\begin{array}{r}0 \\
\$ 0\end{array}$ & $\$ 0$ & $\begin{array}{l}3442.152 \\
\$ 55,074\end{array}$ \\
\hline UC. perEa & 4.00 & $\mathrm{Ea}$ & $\begin{array}{r}10.9 \\
44\end{array}$ & $\begin{array}{l}\$ 53.87 \\
\text { CNPIPE }\end{array}$ & $\begin{array}{l}587,183 \\
\$ 2,349\end{array}$ & $\begin{array}{l}0 \\
\$ 0\end{array}$ & $\begin{array}{r}4225 \\
\$ 16,900\end{array}$ & $\begin{array}{r}0 \\
\$ 0\end{array}$ & $\$ 0$ & $\begin{array}{l}4812,183 \\
\$ 19,249\end{array}$ \\
\hline U.C. per Ea & 2.00 & $\mathrm{Ea}$ & $\begin{array}{r}6 \\
12\end{array}$ & $\begin{array}{l}\$ 53.87 \\
\text { CNPIPE }\end{array}$ & $\begin{array}{r}323.22 \\
\$ 646\end{array}$ & $\$ 0$ & $\begin{array}{r}2200 \\
\$ 4,400\end{array}$ & $\begin{array}{l}0 \\
\$ 0\end{array}$ & $\$ 0$ & $\begin{array}{l}\begin{array}{r}2523.22 \\
\$ 5,046\end{array} \\
\end{array}$ \\
\hline U.c. per Ea & 8.00 & $\mathrm{Ea}$ & $\begin{array}{l}3.2 \\
26\end{array}$ & $\begin{array}{l}\$ 53.87 \\
\text { CNPIPE }\end{array}$ & $\begin{array}{l}172,384 \\
\$ 1,379\end{array}$ & $\$ 0$ & $\begin{array}{r}58 \\
\$ 464\end{array}$ & $\begin{array}{l}0 \\
\$ 0\end{array}$ & $\$ 0$ & $\begin{array}{l}\begin{array}{l}230,384 \\
\$ 1,843\end{array} \\
\end{array}$ \\
\hline UC perEa & 40.00 & $\mathrm{Ea}$ & $\begin{array}{r}6 \\
240\end{array}$ & $\begin{array}{l}\$ 53.87 \\
\text { CNPIPE }\end{array}$ & $\begin{array}{r}32322 \\
\$ 12,929\end{array}$ & $\$$ & $\begin{array}{r}143 \\
\$ 5,720\end{array}$ & $\begin{array}{r}0 \\
\text { \$o }\end{array}$ & $\$ 0$ & $\begin{array}{r}46,22 \\
\$ 18,649\end{array}$ \\
\hline U.C. perEa & 8.00 & $\mathrm{Ea}$ & $\begin{array}{r}2.48 \\
20\end{array}$ & $\begin{array}{l}\$ 53.87 \\
\text { CNPIPE }\end{array}$ & $\begin{array}{l}133.598 \\
\$ 1,069\end{array}$ & $\$ 0$ & $\begin{array}{r}35.5 \\
\$ 284\end{array}$ & \$o & $\$ 0$ & $\begin{array}{l}169.098 \\
\$ 1,353\end{array}$ \\
\hline U. per Ea & 40.00 & Ea & $\begin{array}{r}2.48 \\
99\end{array}$ & $\begin{array}{l}\$ 53.87 \\
\text { CNPIPE }\end{array}$ & $\begin{array}{r}733.598 \\
\$ 5,344\end{array}$ & $\$ 0$ & $\begin{array}{r}35.5 \\
\$ 1,420\end{array}$ & $\$ 0$ & $\$ 0$ & $\begin{array}{r}169.098 \\
\$ 6,764\end{array}$ \\
\hline U.C. per Ea & 4.00 & $\mathrm{Ea}$ & $\begin{array}{r}6 \\
24\end{array}$ & $\begin{array}{l}\text { \$53.87 } \\
\text { CNPIPE }\end{array}$ & $\begin{array}{r}323.22 \\
\$ 1,293\end{array}$ & $\$ 0$ & $\begin{array}{r}700 \\
\$ 2,800\end{array}$ & $\begin{array}{l}0 \\
\$ 0\end{array}$ & $\$ 0$ & $\begin{array}{l}1023,22 \\
\$ 4,093\end{array}$ \\
\hline U. per Lnit & 300.00 & Lnft & $\begin{array}{r}0.4 \\
120\end{array}$ & $\begin{array}{l}\text { \$53.87 } \\
\text { CNPIPE }\end{array}$ & $\begin{array}{r}21.548 \\
\$ 6,464\end{array}$ & $\$ 0$ & $\begin{array}{r}10,15 \\
\$ 3,045\end{array}$ & $\begin{array}{r}0 \\
\$ 0\end{array}$ & $\$ 0$ & $\begin{array}{r}31.698 \\
\$ 9,509\end{array}$ \\
\hline
\end{tabular}

11:08:15

Client: C.P.Ischay

Prepared By: A.W. Miller/S. N. Wasle

other TOTAL

MECH
Flanged 150 Actuated Control Valves 4"

MECH
Slip-on Flanges 150\# 4" thru 6"

MECH
Slip-on Flanges 150\# 8" thru 10"

Gasket and Bolt Set 4" thru $6^{4}$
Memo: RSMeans 22111347

MECH
Gasket and Bolt Set 8" thru 10"
Memo: RSMeans 221113.47

$$
\begin{aligned}
& \text { Flex Couplings } 6 \text { "thru 10" } \\
& \text { PECH } \\
& \text { Piping Insulation Installation }
\end{aligned}
$$

4783

0.013
.474

442.152
55,074

19,249

046

843

466.22
18,649

1,353

764

31.698
9,509 
Project Name: RTC Waste Heat Recovery

Project Location: ATR

Estimate Number:7850

Code Description $\quad$ contractor

5.1.5.2 Heat Exchanger Piping

DETAIL ITEM REPORT

lient: C.P.Ischay

Prepared By: A. W. Miller/S. N. Wasley
Estimate Type: Class-5

Qty UOM Hrs Resource Labor Equipment Material subcontractor Other TOTAL

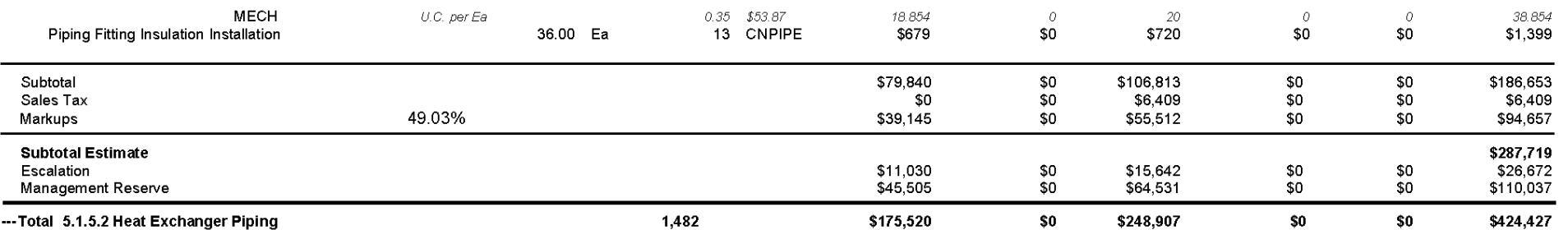

5.1.5.3 Heat Exchanger Pumps \& Misc

\begin{tabular}{|c|c|c|c|c|c|c|c|c|c|c|c|}
\hline $\begin{array}{l}\text { Heat Exchanger Pumps (Prinary } \\
\text { Memo: RSMeans } 2321.23 .13 \text { (5620) }\end{array}$ & UC. per Ea & 2.00 & $\mathrm{Ea}$ & $\begin{array}{l}20 \\
40\end{array}$ & $\begin{array}{l}\text { s5.86 } \\
\text { CNPPE }\end{array}$ & $\begin{array}{r}1077.4 \\
\$ 2,155\end{array}$ & $\begin{array}{l}0 \\
\text { so }\end{array}$ & $\begin{array}{r}\$ 3400 \\
\$ 26,800\end{array}$ & $\begin{array}{l}0 \\
\$ 0\end{array}$ & so & $\begin{array}{r}14477.4 \\
\$ 28,955\end{array}$ \\
\hline $\begin{array}{c}\text { MECH } \\
\text { Heat Exchanger Pumps (Secondary) } \\
\text { Memo: RSMeans 23 21.23.13 (5620) }\end{array}$ & U.c. perEa & 2.00 & Ea & 40 & $\begin{array}{l}\text { s5.867 } \\
\text { CNPPE }\end{array}$ & $\begin{array}{r}1077.4 \\
\$ 2,155\end{array}$ & $\$ 0$ & $\$ 26,800$ & $\$ 0$ & $\$ 0$ & $\begin{array}{r}14477.4 \\
\$ 28,955\end{array}$ \\
\hline Expansion Tank 300 Gal $M E C H$ & U. perEa & & $\mathrm{Ea}$ & $\begin{array}{l}10 \\
10\end{array}$ & $\begin{array}{l}553.87 \\
\text { CNPIPE }\end{array}$ & $\begin{array}{l}538.7 \\
\$ 539\end{array}$ & 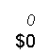 & $\begin{array}{r}8250 \\
\$ 8,250\end{array}$ & $\begin{array}{r}0 \\
\$ 0\end{array}$ & \$0 & $\begin{array}{r}8790.7 \\
\$ 8,789\end{array}$ \\
\hline
\end{tabular}

BEA

11:08:15 
Project Name: RTC Waste Heat Recovery

Project Location: ATR

Code Description contractor

5.1.5.3 Heat Exchanger Pumps \& INIsc

\begin{tabular}{|c|c|c|c|c|c|c|c|c|}
\hline Propylene Glycol Solution $\mathrm{MECH}$ & $\begin{array}{l}\text { U.C. per Gallons } \\
1,822.00 \text { Gallons }\end{array}$ & $\begin{array}{l}0.1 \quad \$ 53.87 \\
182 \quad \text { CNPIPE }\end{array}$ & $\begin{array}{r}5,387 \\
\$ 9,815\end{array}$ & $\stackrel{0}{\$ 0}$ & $\begin{array}{r}26.52 \\
\$ 48,319\end{array}$ & $\stackrel{0}{\$ 0}$ & $\stackrel{0}{\$ 0}$ & $\begin{array}{r}31.907 \\
\$ 58,135\end{array}$ \\
\hline Total 5.1.5.3 Heat Exchanger Pump & & & $\$ 32,236$ & so & $\$ 256,729$ & so & so & $\$ 288,965$ \\
\hline
\end{tabular}

5.2.1 Provide Safequard and Security Support (Plan Development)

Memo: Allowance is based on contact with the ATR Security SME, R. L. Bumham.

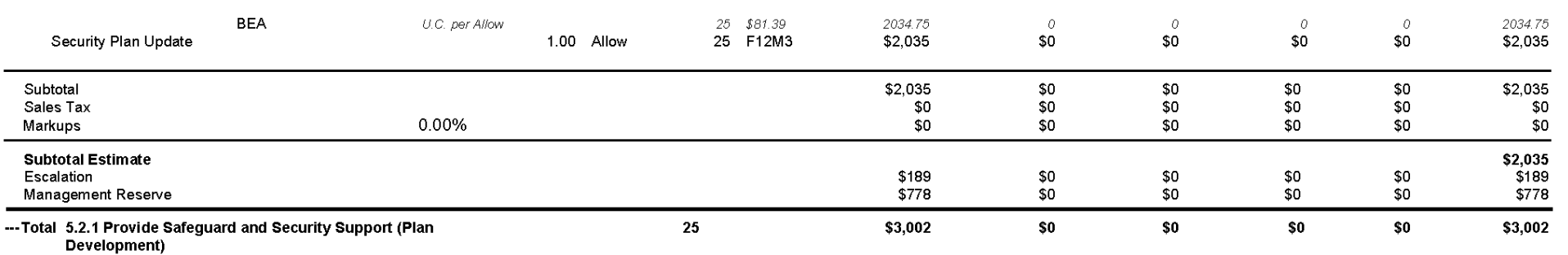

5.2.2Provide Operations Support for Outages

Memo: This actìnty is to provide operations support for rip out and installation
BEA Ua per Allow
BEA
U.C. per Allow

SYS MECHANIC

1.00 Allow

$\begin{array}{ll}40 & 568.47 \\ 40 & \text { U } 29 G B\end{array}$

2738.8
$\$ 2,739$

$\$ 0$

\$o

$\$ 0$

$\$ 0$

2738.8
$\$ 2,739$
11:08:15
Cost Estimating 
Project Name: RTC Waste Heat Recovery

Project Location: ATR

Primate Number: 7850

Code Description $\quad$ Contractor

5.2.2 Provide operations support ror outages

Memo: This actĩüy is to provide operations support for rip out and installation

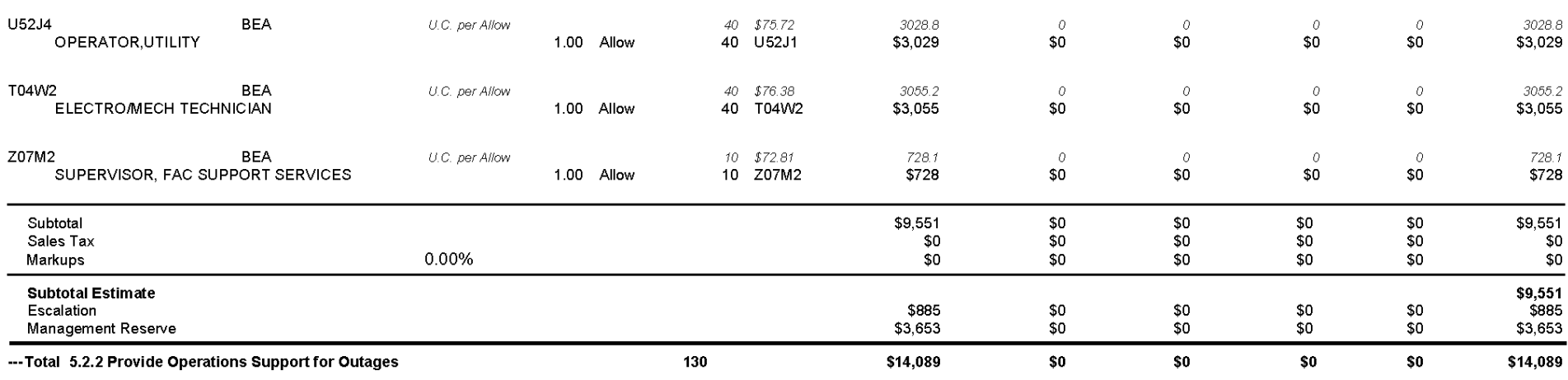

5.2.3 Provide Radcon Support During Sub-k Activities

Perthe team's direction radcon will be reenied during all subcontat activites.

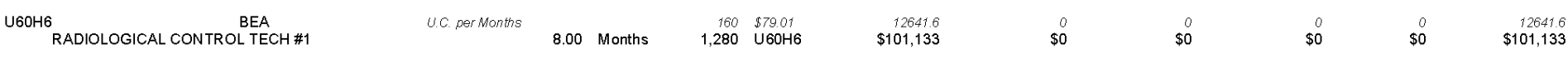

DETAIL ITEM REPORT

C. P.Ischay

Prepared By: A.W. Miller/S. N. Wasley

Estimate Type: Class-5
Equipment Material Subcontractor other TOTAL

(n)

(n)


Project Name: RTC Waste Heat Recovery

Project Location: ATR

Code Description

.2.3 Provide Radcon Support Dunng Sub-k Actuvilues

\begin{tabular}{|c|c|c|c|c|c|c|c|c|c|}
\hline $\begin{array}{l}\text { U60H6 } \\
\text { RADIOLOGICAL CONTROL TECH \#2 }\end{array}$ & U. per Months & 8.00 Months & $\begin{aligned} 160 & \$ 79.01 \\
1,280 & \cup 60 \mathrm{H} 6\end{aligned}$ & $\begin{array}{r}12641,6 \\
\$ 101,133\end{array}$ & $\begin{array}{r}0 \\
\$ 0\end{array}$ & \$o & $\begin{array}{r}0 \\
\text { \$o }\end{array}$ & $\begin{array}{r}0 \\
\$ 0\end{array}$ & $\begin{array}{r}12641.6 \\
\$ 101,133\end{array}$ \\
\hline rotal 5.2.3 $\mathrm{Pro}$ & & & & $\$ 298,371$ & $\$ 0$ & so & so & so & $\$ 298,371$ \\
\hline
\end{tabular}

\subsubsection{Provide for Security/Escorts/Guard Service}

Memo: This is based on 4 days per week, 10 hours per day during the subcontractor work scope.

\begin{tabular}{|c|c|c|c|c|c|c|c|c|c|c|}
\hline $\begin{array}{l}\text { UEGM } \\
1 \text { st SECURITY POLICE OFFICER II (SPO II) }\end{array}$ & U.C. per Months & 8.00 & Months & $\begin{aligned} 160 & \$ 53.52 \\
1,280 & \text { U96M }\end{aligned}$ & $\begin{array}{r}8563.2 \\
\$ 68,506\end{array}$ & $\$ 0$ & $\begin{array}{l}0 \\
\$ 0\end{array}$ & $\begin{array}{r}0 \\
\$ 0\end{array}$ & $\$ 0$ & $\begin{array}{r}8563.2 \\
\$ 68,506\end{array}$ \\
\hline $\begin{array}{l}\text { U96M } \\
\text { BEA } \\
2 \text { nd SECURITY POLICE OFFICER \| (SPO II) }\end{array}$ & U.C. per Months & 8.00 & Months & $\begin{aligned} 160 & \$ 53.52 \\
1,280 & \text { U96M } 4\end{aligned}$ & $\begin{array}{r}8563.2 \\
\$ 68,506\end{array}$ & $\stackrel{0}{\$ 0}$ & $\stackrel{0}{\$ 0}$ & $\$ 0$ & $\stackrel{0}{\$ 0}$ & $\begin{array}{r}8563.2 \\
\$ 68,506\end{array}$ \\
\hline $\begin{array}{l}\text { Subtotal } \\
\text { Sales Tax } \\
\text { Markups }\end{array}$ & $0.00 \%$ & & & & $\begin{array}{r}\$ 137,011 \\
\$ 0 \\
\$ 0\end{array}$ & $\begin{array}{l}\$ 0 \\
\$ 0 \\
\$ 0\end{array}$ & $\begin{array}{l}\$ 0 \\
\$ 0 \\
\$ 0\end{array}$ & $\begin{array}{l}\$ 0 \\
\$ 0 \\
\$ 0\end{array}$ & $\begin{array}{l}\$ 0 \\
\$ 0 \\
\$ 0\end{array}$ & $\begin{array}{r}\$ 137,011 \\
\$ 0 \\
\$ 0\end{array}$ \\
\hline $\begin{array}{l}\text { Subtotal Estimate } \\
\text { Escalation } \\
\text { Management Reserve }\end{array}$ & & & & & $\begin{array}{l}\$ 12,701 \\
\$ 52,399\end{array}$ & $\begin{array}{l}\$ 0 \\
\$ 0\end{array}$ & $\begin{array}{l}\$ 0 \\
\$ 0\end{array}$ & $\begin{array}{l}\$ 0 \\
\$ 0\end{array}$ & $\begin{array}{l}\$ 0 \\
\$ 0\end{array}$ & $\begin{array}{r}\$ 137,011 \\
\$ 12,701 \\
\$ 52,399\end{array}$ \\
\hline --Total 5.2.4 Provide for Security/Escorts/Gu & & & & & $\$ 202,111$ & \$o & $\$ 0$ & \$o & so & $\$ 202,111$ \\
\hline
\end{tabular}


Project Name: RTC Waste Heat Recovery

Project Location: ATR

\section{Code Description Contractor}

5.3 Provide For Prolect Specilic Tranning - Subcontracior
DETAIL ITEM REPORT

Client: C.P.Ischay

Prepared By: A. W. Miller/S. N. Wasley

Estimate Type: Class-5

\section{aterial Subcontractor other}

TOTAL

Memo: Training allowance is based on estimator judgment and review of the INL Training List it is assumed that the general type craft currently engaged with work at the INL will only require

\begin{tabular}{|c|c|c|c|c|c|c|c|c|c|c|}
\hline $\begin{array}{l}G \in N \\
\text { CARPENTERS - Subcontract Project Supervisor }\end{array}$ & U.c. per craft & $\begin{array}{l}\text { NM80 } \\
1.00 \stackrel{\text { Craft }}{ }\end{array}$ & $\begin{array}{l}10 \\
10\end{array}$ & $\begin{array}{l}\$ 49.04 \\
\text { CNCARPF }\end{array}$ & $\begin{array}{l}490.4 \\
\$ 490\end{array}$ & $\$ 0$ & $\begin{array}{l}0 \\
\$ 0\end{array}$ & $\begin{array}{l}0 \\
\$ 0\end{array}$ & $\begin{array}{l}0 \\
\$ 0\end{array}$ & $\begin{array}{l}490.4 \\
\$ 490\end{array}$ \\
\hline $\begin{array}{l}\text { GEN } \\
\text { CARPENTERS - CONCRETE - FOREMAN }\end{array}$ & U. per craft & $1.00 \stackrel{\text { NM80 }}{\text { Craft }}$ & $\begin{array}{l}10 \\
10\end{array}$ & $\begin{array}{l}\$ 49.35 \\
\text { CNCARPCF }\end{array}$ & $\begin{array}{l}493.5 \\
\$ 494\end{array}$ & $\$ 0$ & $\$ 0$ & $\begin{array}{l}0 \\
\$ 0\end{array}$ & $\$ 0$ & $\begin{array}{l}493.5 \\
\$ 494\end{array}$ \\
\hline CARPENTERS & U. per craft & $3.00 \stackrel{\mathrm{NM} 80}{\mathrm{Craft}}$ & $\begin{array}{r}10 \\
30\end{array}$ & $\begin{array}{l}\$ 46.92 \\
\text { CNCARP }\end{array}$ & $\begin{array}{r}469.2 \\
\$ 1,408\end{array}$ & $\$ 0$ & \$o & $\$ 0$ & $\$ 0$ & $\$ 41,408$ \\
\hline CEMENT MASONS & U. per craft & $3.00 \stackrel{\text { NM80 }}{\text { Craft }}$ & $\begin{array}{r}10 \\
30\end{array}$ & $\begin{array}{l}\$ 45.28 \\
\text { CNCEM }\end{array}$ & $\begin{array}{r}452.8 \\
\$ 1,358\end{array}$ & $\$ 0$ & $\$ 0$ & $\begin{array}{l}0 \\
\$ 0\end{array}$ & $\$ 0$ & $\$ 41,358$ \\
\hline IRONWORKERS & U.C. per Craft & $2.00 \stackrel{\mathrm{NM} 80}{\text { Craft }}$ & $\begin{array}{c}10 \\
20\end{array}$ & $\begin{array}{l}\$ 57.47 \\
\text { CNIRON }\end{array}$ & $\$ 1,149$ & $\$ 0$ & $\$ 0$ & $\$ 0$ & $\$ 0$ & $\$ 1,149$ \\
\hline $\begin{array}{l}\text { STEEL } \\
\text { IRONWORKERS - FOREMAN }\end{array}$ & U.c. per craft & $\begin{array}{l}\mathrm{NM80} \\
1.00 \stackrel{\text { Craft }}{ }\end{array}$ & $\begin{array}{c}10 \\
10\end{array}$ & $\begin{array}{l}\$ 58.32 \\
\text { CNIRONF }\end{array}$ & $\begin{array}{l}583.2 \\
\$ 583\end{array}$ & $\$ 0$ & $\$ 0$ & $\$ 0$ & $\$ 0$ & $\$ 58383$ \\
\hline LABORERS & U.C. per Craft & $4.00 \stackrel{\text { NM80 }}{\text { Craft }}$ & $\begin{array}{r}10 \\
40\end{array}$ & $\begin{array}{l}\$ 44.07 \\
\text { CNLAB }\end{array}$ & $\begin{array}{r}440.7 \\
\$ 1,760\end{array}$ & \$o & $\$ 0$ & $\$ 0$ & $\$ 0$ & $\$ 440.1$ \\
\hline LABORERS - GF & U.C. per craft & $1.00 \stackrel{\text { NM80 }}{\text { Craft }}$ & $\begin{array}{r}10 \\
10\end{array}$ & $\begin{array}{l}\$ 47.55 \\
\text { CNLABGF }\end{array}$ & $\begin{array}{l}475.5 \\
\$ 476\end{array}$ & $\$ 0$ & $\$ 0$ & $\$ 0$ & $\$ 0$ & $\begin{array}{l}475.5 \\
\$ 476\end{array}$ \\
\hline OPERATORS-CRANES GEN & U.C. per Craft & $1.00 \stackrel{\text { NM80 }}{\text { Craft }}$ & $\begin{array}{l}10 \\
10\end{array}$ & $\begin{array}{l}540.36 \\
\text { CNOPRCR }\end{array}$ & $\begin{array}{l}483.6 \\
\$ 484\end{array}$ & \$o & $\begin{array}{r}0 \\
\$ 0\end{array}$ & $\begin{array}{r}0 \\
\$ 0\end{array}$ & $\$ 0$ & $\begin{array}{l}483.6 \\
\$ 484\end{array}$ \\
\hline DPERATORS - Misc $\quad$ GEN & U.C. per Craft & $\begin{array}{l}\text { NM80 } \\
1.00 \text { Craft }\end{array}$ & $\begin{array}{l}10 \\
10\end{array}$ & $\begin{array}{l}\$ 46.82 \\
\text { CNOPRL1 }\end{array}$ & $\begin{array}{l}4682 \\
5468\end{array}$ & $\$ 0$ & $\$ 0$ & $\$ 0$ & so & $\$ 468$ \\
\hline
\end{tabular}


Project Name: RTC Waste Heat Recovery

Project Location: ATR

Pstimate Number: 7850

Code Description

5.3 Provide For Prolect Specinc traning - Subcontractor

\begin{tabular}{|c|c|c|c|c|c|c|c|c|c|c|}
\hline $\begin{array}{l}\text { Subtotal } \\
\text { Sales Tax } \\
\text { Markups }\end{array}$ & & $40.26 \%$ & & & $\begin{array}{r}\$ 9,126 \\
\$ 0 \\
\$ 3,674\end{array}$ & $\begin{array}{l}\$ 0 \\
\$ 0 \\
\$ 0\end{array}$ & $\begin{array}{l}\$ 0 \\
\$ 0 \\
\$ 0\end{array}$ & $\begin{array}{l}\$ 0 \\
\$ 0 \\
\$ 0\end{array}$ & $\begin{array}{l}\$ 0 \\
\$ 0 \\
\$ 0\end{array}$ & $\begin{array}{r}\$ 9,126 \\
\$ 0 \\
\$ 3,674\end{array}$ \\
\hline $\begin{array}{l}\text { Subtotal Estimate } \\
\text { Escalation } \\
\text { Management Reserve }\end{array}$ & & & & & $\begin{array}{l}\$ 1,186 \\
\$ 4,895\end{array}$ & $\begin{array}{l}\$ 0 \\
\$ 0\end{array}$ & $\begin{array}{l}\$ 0 \\
\$ 0\end{array}$ & $\$ 0$ & $\begin{array}{l}\$ 0 \\
\$ 0\end{array}$ & $\begin{array}{r}\$ 12,799 \\
\$ 1,186 \\
\$ 4,895\end{array}$ \\
\hline $\begin{array}{l}\text { Total } 5.3 \text { Provide Fo } \\
\text { Subcontractor }\end{array}$ & & & & & $\$ 18,880$ & \$0 & \$o & $\$ 0$ & $\$ 0$ & $\$ 18,880$ \\
\hline
\end{tabular}

DETAIL ITEM REPORT

C.P.Ischay

Prepared By: A.W.Miller/S. N. Wasley

Estimate Type: Class-5

Subcontractor Other TOTAL

Equipment

Tot s.3 Prowde For Project Specific Training

Qty UOM Hrs Resource Labor

\subsection{Provide for 10 CFR 851 Requirements}

Memo: This requirement is based on any subcontract exceeding 30 days in duration. This includes all fower tier subcontractors even when less than 30 days in duration. This is per $R$. Strong,
BEA Construction Management

Subcontractor Medical Allowances - See Below
Memo: Based on direction from D. Inskeep of the SWO BEA costs for 10 CFR 851 per covered employee are identified below.

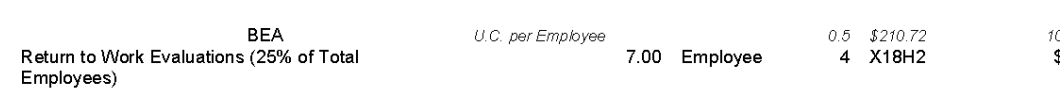

$\$ 0$

$\$ 0$

0.0
$\$ 0$

$\$ 0$

$\$ 0$

0.01
$\$ 0$

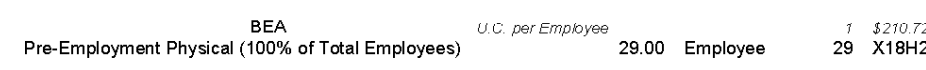

Exit Physical (5\% of Total Employees)

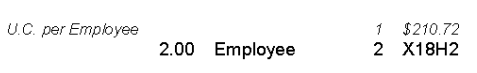

BEA
Employee Assistance Program Visit (10\% of Total
Employees)

$\begin{array}{rrrr}\text { U.C. per Emplioye日 } & & 1 & \$ 210.72 \\ 3.00 & \text { Employee } & 3 & \mathrm{X} 18 \mathrm{H} 2\end{array}$

105.36
$\$ 738$

so $\quad$ so

$\begin{array}{rrr}0 & 0 & 10536 \\ \$ 0 & \$ 0 & \$ 738\end{array}$

EA

BEA
05/11/2012 $\quad 11: 08: 15$

Cost Estimating 
Project Name: RTC Waste Heat Recovery

Project Location: ATR
DETAIL ITEM REPORT

Client: C.P.Ischay

Prepared By: A. W. Miller/S. N. Wasley

Estimate Type: Class-5

\section{Code Description Contractor \\ Qty UOM Hrs Resource Labor \\ Equipment \\ Material Subcontractor \\ Other \\ TOTAL} Memo: This requirement is based on any subcontract exceeding 30 days in duration. This includes all fower tier subcontractors even when less than 30 days in duration. This is per $R$. Strong,

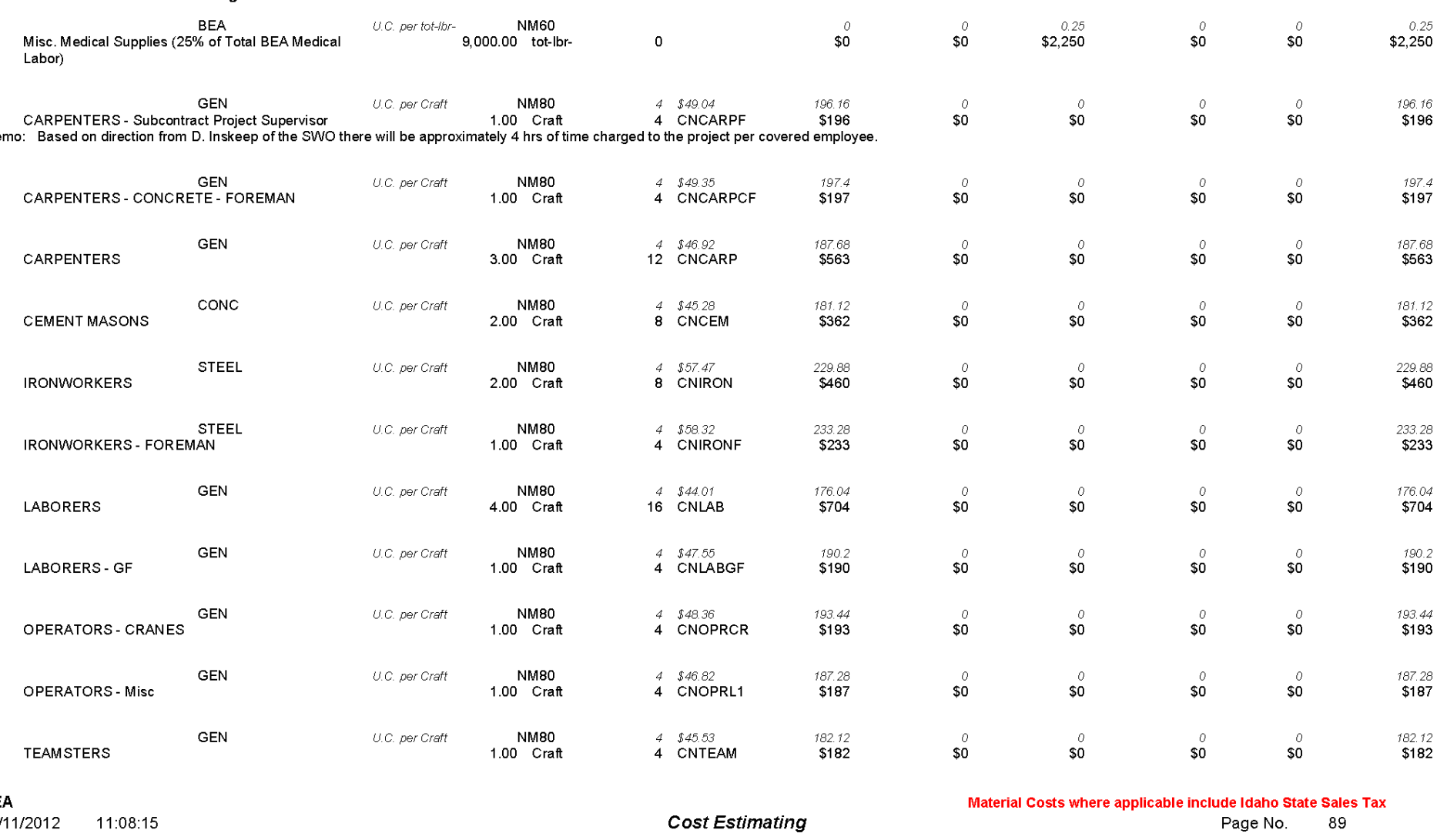

Cost Estimating

$$
\text { Page No. } \quad 89
$$


Project Name: RTC Waste Heat Recovery

Project Location: $A T R$

Code Description $\quad \underline{\text { contractor }}$

5.4 Provide for 10 CFR 851 Requirements

Memo: This requirement is based on any subcontract exceeding 30 days in duration. This includes all lower tier subcontractors even when less than 30 days in duration. This is per $R$. Strong,

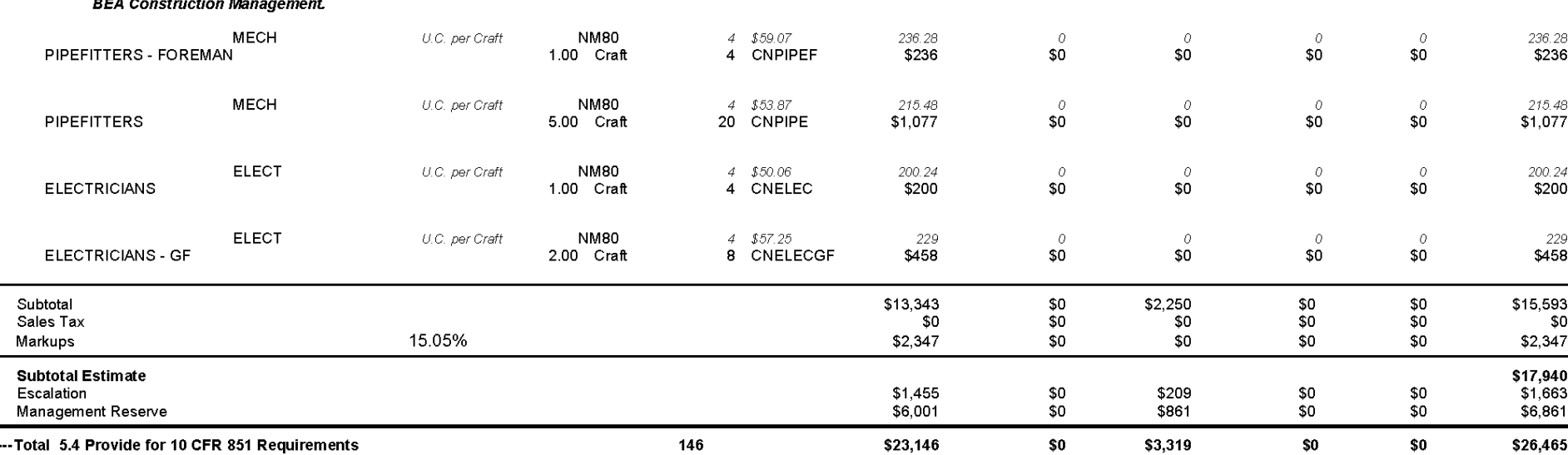

\subsubsection{Provide AE Field Support/oversight during Execution - Level of Effort (LOE)}

Memo: Based on estimator judgment and the project team to account for all engineering activities during the execution phase.

\begin{tabular}{|c|c|c|c|c|c|c|c|c|c|c|c|}
\hline $\begin{array}{l}\text { EE^ } \\
\text { Provide AE Field Support and Answer Civil/Struct } \\
\text { Questions }\end{array}$ & Uo. porwook & 32.00 & weeks & $256^{s}$ & $\begin{array}{l}8148.58 \\
\text { E04W1 }\end{array}$ & $\begin{array}{l}1199646 \\
\$ 38,036\end{array}$ & \$o & $\begin{array}{l}0 \\
\$ 0\end{array}$ & $\$$ & $\$ 0$ & $\begin{array}{r}1188,64 \\
\$ 38,036\end{array}$ \\
\hline $\begin{array}{l}\text { BEA } \\
\text { Provide AE Field Support and Answer Electrical }\end{array}$ & Uc. perweeks & 32.00 & weeks & $\begin{array}{r}10 \\
320\end{array}$ & $\begin{array}{l}\$ 118.73 \\
\text { E06W1 }\end{array}$ & $\begin{array}{r}1187.3 \\
\$ 37,994\end{array}$ & \$o & $\begin{array}{l}0 \\
\text { \$0 }\end{array}$ & $\begin{array}{r}0 \\
\text { so }\end{array}$ & $\begin{array}{r}0 \\
\text { so }\end{array}$ & $\begin{array}{r}1187.3 \\
\$ 37,994\end{array}$ \\
\hline
\end{tabular}


Project Name: RTC Waste Heat Recovery

Project Location: ATR

Code Description

5.5.1 Provide AE Fleld Supportioversignt aunna Execution - Level or Enort LLO
BEA
Provide AE Field Support and Answer Mechanica
U.c. perweoks
32.00 weeks
$\begin{array}{rl}15 & \$ 119.29 \\ 480 & \mathrm{E} 11 \mathrm{~W} 1\end{array}$
$\$ 57,259$

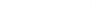

$\begin{array}{rrrrr}0 & 0 & 0 & 0 & 1789,35 \\ \$ 0 & \$ 0 & \$ 0 & \$ 0 & \$ 57,259\end{array}$

Subtotal

Markups

$0.00 \%$

$\$ 133,289$

289
$\$ 0$
$\$ 0$

Subtotal Estimat

Management Reserv

--Total 5.5.1 Provide AE Field Supportoversight during

\begin{tabular}{|c|c|c|c|c|c|}
\hline $\begin{array}{l}\$ 12,356 \\
\$ 50,976\end{array}$ & $\$ 00$ & $\$_{\$ 0}$ & 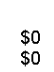 & $\begin{array}{l}\$ 0 \\
\$ 0\end{array}$ & $\begin{array}{r}\mathbf{\$ 1 3 3 , 2 8 9} \\
\$ 12,356 \\
\$ 50,976\end{array}$ \\
\hline & so & \$0 & $\$ 0$ & $\$ 0$ & \\
\hline
\end{tabular}

\subsubsection{Provide AE Support Oversight during Execution - Level of Effort (LOE)}

Memo: Based on estimator judgment to provide managmenet oversite to the engineering group.

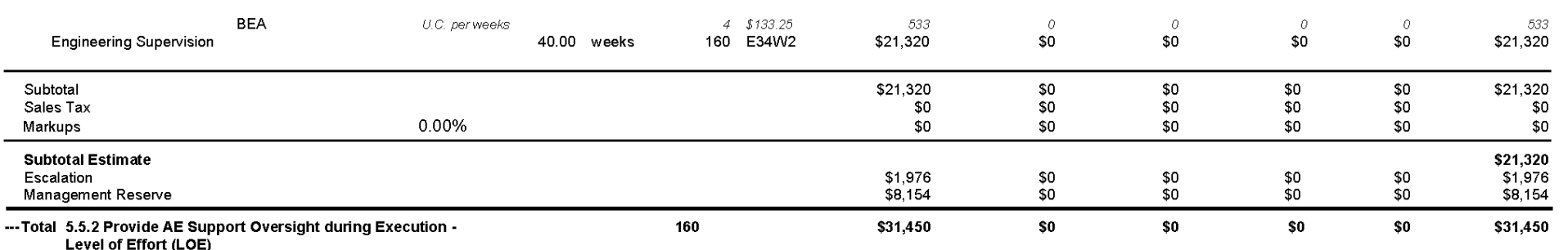


Project Name: RTC Waste Heat Recovery

Project Location: ATR

Estimate Number: 7850

Code Description $\quad$ Contractor

6.0 Environmental Assessment

Allowance is based on direction from the project team

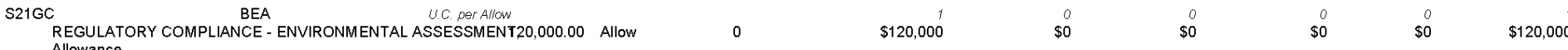

DETAIL ITEM REPORT

Client: C.P.Ischay

Estimate Type: Class-5

Qty UOM Hrs Resource Labor Equipment Material Subcontractor Other TOTAL
Prepared By: A.W.Miller/S. N. Wasley

\begin{tabular}{|c|c|c|c|c|c|c|c|}
\hline $\begin{array}{l}\text { Subtotal } \\
\text { Sales Tax } \\
\text { Markups }\end{array}$ & $0.00 \%$ & $\begin{array}{r}\$ 120,000 \\
\$ 0 \\
\$ 0 \\
\end{array}$ & $\begin{array}{l}\$ 0 \\
\$ 0 \\
\$ 0 \\
\end{array}$ & $\begin{array}{l}\$ 0 \\
\$ 0 \\
\$ 0 \\
\end{array}$ & $\begin{array}{l}\$ 0 \\
\$ 0 \\
\$ 0 \\
\end{array}$ & $\begin{array}{l}\$ 0 \\
\$ 0 \\
\$ 0 \\
\end{array}$ & $\begin{array}{r}\$ 120,000 \\
\$ 0 \\
\$ 0 \\
\end{array}$ \\
\hline $\begin{array}{l}\text { Subtotal Estimate } \\
\text { Escalation } \\
\text { Management Reserve }\end{array}$ & & $\begin{array}{r}\$ 6,672 \\
\$ 44,335\end{array}$ & $\begin{array}{l}\$ 0 \\
\$ 0\end{array}$ & $\begin{array}{l}\$ 0 \\
\$ 0\end{array}$ & $\begin{array}{l}\$ 0 \\
\$ 0\end{array}$ & $\begin{array}{l}\$ 0 \\
\$ 0\end{array}$ & $\begin{array}{r}\$ 120,000 \\
\$ 6,672 \\
\$ 44,335\end{array}$ \\
\hline
\end{tabular}

7.1 Preventative Maintenance (PM) Plan Development

Memo: Based on prior discussions with ATR Plant Engineering

\begin{tabular}{|c|c|c|c|c|c|c|c|c|c|c|c|}
\hline $\begin{array}{l}\text { EEA } \\
\text { Modify and Update Existing Preventative } \\
\text { Maintenance (PM's) Plans (Planner Support) }\end{array}$ & U.C. per flans & 12.00 & Plans & $\begin{array}{r}10 \\
120\end{array}$ & $\begin{array}{l}\$ 79.42 \\
\text { F10GB }\end{array}$ & $\begin{array}{r}794.2 \\
\$ 9,530\end{array}$ & $\begin{array}{l}0 \\
\$ 0\end{array}$ & $\begin{array}{r}25 \\
\$ 300\end{array}$ & $\begin{array}{c}0 \\
\$ 0\end{array}$ & $\$ 0$ & $\begin{array}{r}819.2 \\
\$ 9,830\end{array}$ \\
\hline $\begin{array}{l}\text { BEA } \\
\text { Modify and Update Existing Preventative } \\
\text { Maintenance (PM's) Plans (Engineer Support) }\end{array}$ & U. per plans & 12.00 & Plans & $\begin{array}{l}100 \\
120\end{array}$ & $\begin{array}{l}\$ 101.57 \\
E 48 W 1\end{array}$ & $\begin{array}{r}1015.7 \\
\$ 12,188\end{array}$ & \$o & $\begin{array}{r}25 \\
\$ 300\end{array}$ & \$o & so & $\begin{array}{r}1040.7 \\
\$ 12,488\end{array}$ \\
\hline $\begin{array}{l}\text { BEA } \\
\text { Develop New PMs for the Recovery System (Planner } \\
\text { Supvurl) }\end{array}$ & UC per Plans & 10.00 & Plans & $\begin{array}{r}25 \\
250\end{array}$ & $\begin{array}{l}\$ 79.42 \\
\text { F10GB }\end{array}$ & $\begin{array}{r}1985.5 \\
\$ 19,855\end{array}$ & $\begin{array}{l}0 \\
\$ 0\end{array}$ & $\begin{array}{r}25 \\
\$ 250\end{array}$ & $\begin{array}{r}0 \\
\$ 0\end{array}$ & \$o & \\
\hline
\end{tabular}


Project Name: RTC Waste Heat Recovery

Project Location: ATR

Estimate Number: 7850

\section{Code Description Contractor}

7.1 Preventatlve Wlaintenance (PV) Plan Development

Memo: $\quad$ Based on prior discussions with ATR Plant Engineering

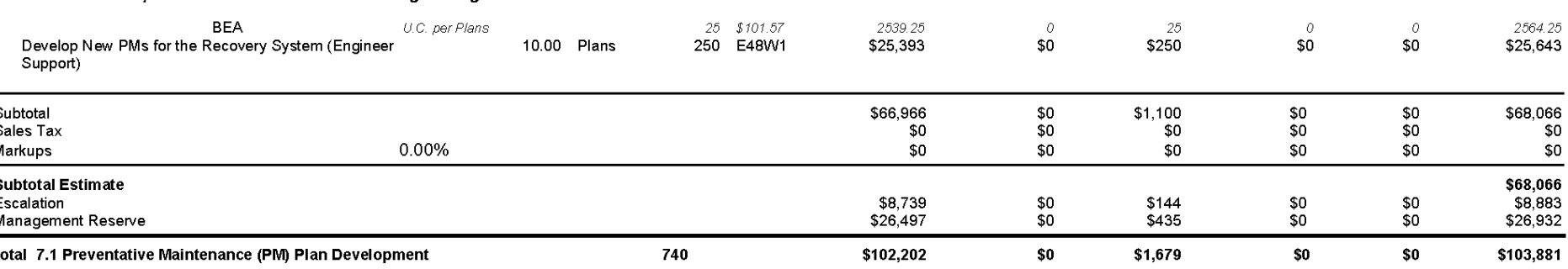

点

7.2 Develop Operating Procedures, Manuals \& Documents

Memo: $\quad$ Based on prior discussions with ATR Plant Engineering

\begin{tabular}{|c|c|c|c|c|c|c|c|c|c|c|}
\hline $\begin{array}{l}\text { BEA } \\
\text { Development of new Operating Procedures }\end{array}$ & U. per $E A$ & $\begin{array}{l}\mathrm{NM} 60 \\
6.00 \mathrm{EA}\end{array}$ & $\begin{array}{r}60 \\
360\end{array}$ & $\begin{array}{l}\$ 121.94 \\
\text { E32WW1 }\end{array}$ & $\begin{array}{r}7316.4 \\
\$ 43,898\end{array}$ & $\$ 0$ & $\begin{array}{r}25 \\
\$ 150\end{array}$ & $\begin{array}{r}0 \\
\$ 0\end{array}$ & $\$ 0$ & $\begin{array}{r}7341.4 \\
\$ 44,048\end{array}$ \\
\hline $\begin{array}{l}\text { BEA } \\
\text { Update \& Revise Current Operating Procedures - OMM }\end{array}$ & U. perEA & $12.00 \begin{array}{l}\mathrm{NM60} \\
\mathrm{EA}\end{array}$ & $\begin{array}{r}10 \\
120\end{array}$ & $\begin{array}{l}\$ 121.94 \\
\text { E32W1 }\end{array}$ & $\begin{array}{r}1219.4 \\
\$ 14,633\end{array}$ & $\$ 0$ & $\begin{array}{r}25 \\
\$ 300\end{array}$ & $\$ 0$ & $\$ 0$ & $\$ 1244,44$ \\
\hline $\begin{array}{l}\text { BEA } \\
\text { Update \& Revise Current Operating Procedures - DOP }\end{array}$ & U.C. per EA & $6.00 \stackrel{\mathrm{NM} 60}{\mathrm{EA}}$ & $\begin{array}{l}10 \\
60\end{array}$ & $\begin{array}{l}\$ 127.94 \\
\text { E32W1 }\end{array}$ & $\begin{array}{r}1219.4 \\
\$ 7,316\end{array}$ & $\$ 0$ & $\$ 150$ & $\$ 0$ & $\begin{array}{l}0 \\
\$ 0\end{array}$ & $\$ 7,466$ \\
\hline $\begin{array}{l}\text { BEA } \\
\text { Update \& Revise Current Operating Procedures - AOP }\end{array}$ & U.c. por EA & $8.00 \stackrel{\mathrm{NM60}}{\mathrm{EA}}$ & $\begin{array}{l}10 \\
80\end{array}$ & $\begin{array}{l}\$ 121.01 \\
\text { E32W1 }\end{array}$ & $\begin{array}{r}1210.1 \\
\$ 9,755\end{array}$ & $\$ 0$ & $\begin{array}{r}25 \\
\$ 200\end{array}$ & $\$ 0$ & $\$ 0$ & $\$ 9,955$ \\
\hline $\begin{array}{l}\text { BEA } \\
\text { Update \& Revise Current Operating Procedures - RP }\end{array}$ & UC. per $E A$ & $\begin{aligned} & \mathrm{NM60} \\
& 3.00 \stackrel{\mathrm{EA}}{ }\end{aligned}$ & $\begin{array}{l}10 \\
30\end{array}$ & $\begin{array}{l}\$ 121.94 \\
\text { E32W1 }\end{array}$ & $\begin{array}{r}1219.4 \\
\$ 3,658\end{array}$ & $\begin{array}{c}0 \\
\$ 0\end{array}$ & $\begin{array}{r}25 \\
\$ 75\end{array}$ & $\begin{array}{l}0 \\
\$ 0\end{array}$ & $\begin{array}{l}0 \\
\$ 0\end{array}$ & $\begin{array}{r}1244,4 \\
\$ 3,733\end{array}$ \\
\hline
\end{tabular}


Project Name: RTC Waste Heat Recovery

Project Location: ATR

Code Description

2 Develoo operating Procedures, Manuals \& Documents

Memo: Based on prior discussions with ATR Plant Engineering

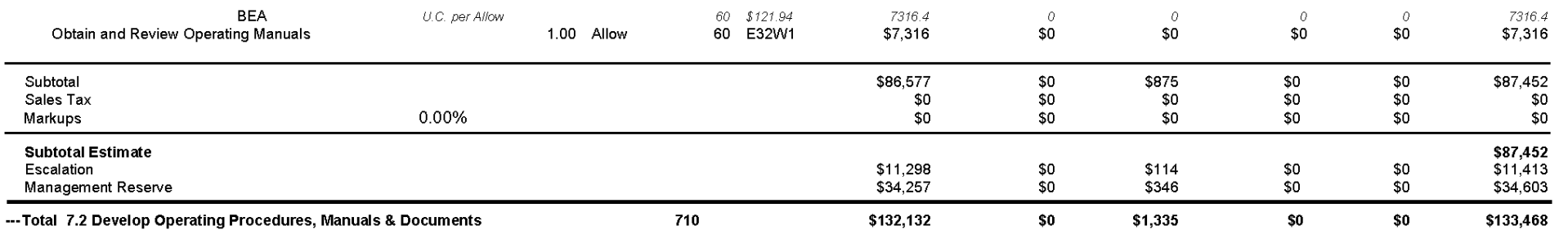

\subsection{Prepare Commissioning/so Test Plan}

Memo: Allowance is based on a group of SME's to develop the so test plan, including comment and review cycle.

E11W4 BEA

E27W4 OTHER ENGINEERING BEA

EESW
ELECTRICAL ENGINEERING

E32W1 ${ }_{\text {SYSTEM/SSC ENGINEER }}^{\text {BEA }}$

E48W4 OPERATIONS ENGINEER, GENERAL

EEA
E35W2
INSTRUMENTATION AND CONTROLS

uc. pertirs

$30.00 \mathrm{Hrs}$

$\begin{array}{rr}1 & \$ 119.29 \\ 30 & \text { E11W1 }\end{array}$

119.29
$\$ 3,579$

Client:

Prepared By: A. W. Miller/S. N. Wasley

Estimate Type: Class-5

Equipment Material Subcontractor Other TOTAL 
Project Name: RTC Waste Heat Recovery

Project Location: ATR

\section{Code Description contractor}

7.3 Prepare commissioninaso Test plan

Memo: Allowance is based on a group of SME's to develop the SO test plan, including comment and review cycle.

\begin{tabular}{|c|c|c|c|c|c|c|c|c|c|c|}
\hline $\begin{array}{l}\text { Z04WA } 4 \text { MANAGER, SCIIENG FUNCTION } \\
\text { MANAG }\end{array}$ & UC. perthrs & 15.00 & Hrs & $\begin{array}{rl}1 & \$ 173.21 \\
15 & \mathrm{Z} 04 \mathrm{~W} 4\end{array}$ & $\begin{array}{r}173.21 \\
\$ 2,598\end{array}$ & $\$ 0$ & $\begin{aligned} 0 \\
\$ 0\end{aligned}$ & $\begin{array}{l}0 \\
\$ 0\end{array}$ & $\$ 0$ & $\begin{array}{r}173.21 \\
\$ 2,598\end{array}$ \\
\hline $\begin{array}{l}\text { Z04W4 } \\
\text { MANAGER, SCIENG FUNCTION }\end{array}$ & UC. per Hrs & 15.00 & Hrs & $\begin{aligned} 1 & \$ 173.21 \\
15 & \text { Z04WU }\end{aligned}$ & $\begin{array}{r}\$ 73.21 \\
\$ 2,598\end{array}$ & $\$ 0$ & $\$ 0$ & $\begin{array}{r}0 \\
\$ 0\end{array}$ & $\stackrel{0}{\$ 0}$ & $\begin{array}{r}\$ 7321 \\
\$ 2,598\end{array}$ \\
\hline $\begin{array}{l}\text { Subtotal } \\
\text { Sales Tax } \\
\text { Markups }\end{array}$ & $0.00 \%$ & & & & $\begin{array}{r}\$ 28,890 \\
\$ 0 \\
\$ 0\end{array}$ & $\begin{array}{l}\$ 0 \\
\$ 0 \\
\$ 0\end{array}$ & $\begin{array}{l}\$ 0 \\
\$ 0 \\
\$ 0\end{array}$ & $\begin{array}{l}\$ 0 \\
\$ 0 \\
\$ 0\end{array}$ & $\begin{array}{l}\$ 0 \\
\$ 0 \\
\$ 0\end{array}$ & $\begin{array}{r}\$ 28,890 \\
\$ 0 \\
\$ 0\end{array}$ \\
\hline $\begin{array}{l}\text { Subtotal Estimate } \\
\text { Escalation } \\
\text { Management Reserve }\end{array}$ & & & & & $\begin{array}{r}\$ 3,770 \\
\$ 11,431\end{array}$ & $\begin{array}{l}\$ 0 \\
\$ 0\end{array}$ & $\begin{array}{l}\$ 0 \\
\$ 0\end{array}$ & $\begin{array}{l}\$ 0 \\
\$ 0\end{array}$ & $\begin{array}{l}\$ 0 \\
\$ 0\end{array}$ & $\begin{array}{l}\$ 28,890 \\
\$ 1,770 \\
\$ 11,431\end{array}$ \\
\hline ---Total 7.3 Prepare Commissioning/ & & & & & $\$ 44,091$ & $\$ 0$ & \$o & so & \$o & $\$ 44,091$ \\
\hline
\end{tabular}

\subsection{SAR Update}

Memo: Allowance only, at this time no information is available for what will be required for the SAR update to the existing facility SAR.

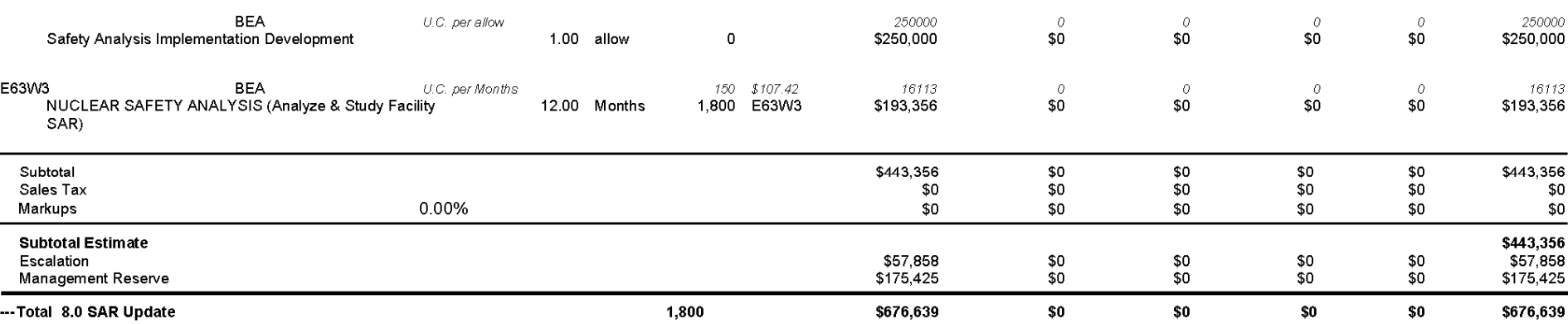

11:08:15 
Project Name: RTC Waste Heat Recovery

Project Location: ATR

\section{Code Description Contractor}

9.0 New System So Testing

Memo: Allowance is based on a group of SME's to perform the SO test This allowance was based on estimator's judgment

BEA
U52J1 UPERATOR,UTILITY (Craft 1)

U52J1 OPERATOR,UTILITY (Craft 2)

EO6W4
ELECTRICAL ENGINEERING

E34W2 2 BEA

EEA
E54W4
OPS SYSTEM ENGR, VITAL SAFETY SYSTEMS

uc. perwks

1.00 Wks

$\begin{array}{ll}40 & \$ 75.72 \\ 40 & \text { U52J1 }\end{array}$

3028.8
$\$ 3,029$

4c. perwts

$\begin{array}{lr}40 & \$ 75.72 \\ 40 & \mathrm{U} 52 \mathrm{~J} 1\end{array}$

$\begin{array}{ll}40 & \$ 118.73 \\ 40 & \text { E06W1 }\end{array}$

3028.8
$\$ 3,029$

U.C. perwhs 1.00 Wks

$\begin{array}{ll}40 & \$ 133.25 \\ 40 & \text { E34W2 }\end{array}$

$\$ 4749.2$

U.C. per WhS

1.00 Wks

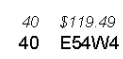

5330
$\$ 5,330$

U.C. per Whs 1.00 Wks

4779.6
$\$ 4,780$

$\begin{array}{ll}\text { U11GB } & \text { BEA } \\ \text { U11GB } & \text { BEA ICIAN (Craft 1) }\end{array}$

U11GB

U16.J1 FIT TER (Craft 1) BEA

U16.J1 FITTER (Craft 2) BEA

$40 \quad 56797$

1.00 Wks

$\begin{array}{ll}40 & \$ 67.97 \\ 40 & \text { U11GB }\end{array}$

2718.8
$\$ 2,719$

$\begin{array}{ll}40 & 567.97 \\ 40 & \mathrm{U} 11 \mathrm{~GB}\end{array}$

2718.8
$\$ 2,719$

$\begin{array}{ll}40 & \$ 67.21 \\ 40 & \mathrm{U} 16 \mathrm{~J} 1\end{array}$

$\begin{array}{ll}40 & 567.21 \\ 40 & \mathrm{U} 16 \mathrm{J1}\end{array}$

$\$ 2688.4$

2688.4
$\$ 2,688$

1.00 Wks

\begin{abstract}
40 visu
\end{abstract}

\section{Client: C. P. Ischay \\ Prepared By: A. W. Miller/S. N. Wasley \\ Estimate Type: Class-5 \\ Equipment Material Subcontractor Other TOTAL}

$\begin{array}{rrrrr}0 & 0 & 0 & 0 & 3028.8 \\ \$ 0 & \$ 0 & \$ 0 & \$ 0 & \$ 3,029 \\ 0 & 0 & 0 & 0 & 3028.8 \\ \$ 0 & \$ 0 & \$ 0 & \$ 0 & \$ 3,029 \\ 0 & 0 & 0 & 0 & 4749.2 \\ \$ 0 & \$ 0 & \$ 0 & \$ 0 & \$ 4,749 \\ 0 & 0 & 0 & 0 & 5330 \\ \$ 0 & \$ 0 & \$ 0 & \$ 0 & \$ 5,330 \\ 0 & 0 & 0 & 0 & 4779.6 \\ \$ 0 & \$ 0 & \$ 0 & \$ 0 & \$ 4,780 \\ 0 & 0 & 0 & 0 & 2718.8 \\ \$ 0 & \$ 0 & \$ 0 & \$ 0 & \$ 2,719 \\ 0 & 0 & 0 & 0 & 2718.8 \\ \$ 0 & \$ 0 & \$ 0 & \$ 0 & \$ 2,719 \\ 0 & 0 & 0 & 0 & 2688.4 \\ \$ 0 & \$ 0 & \$ 0 & \$ 0 & \$ 2,688 \\ 0 & & 0 & 0 & 2688.4 \\ \$ 0 & \$ 0 & \$ 0 & \$ 0 & \$ 2,688\end{array}$


Project Name: RTC Waste Heat Recovery

Project Location: ATR

Estimate Number: 7850

Code Description

9.0 New System So Testing

Memo: Allowance is based on a group of SME's to perform the SO test This allowance was based on estimator's juctong

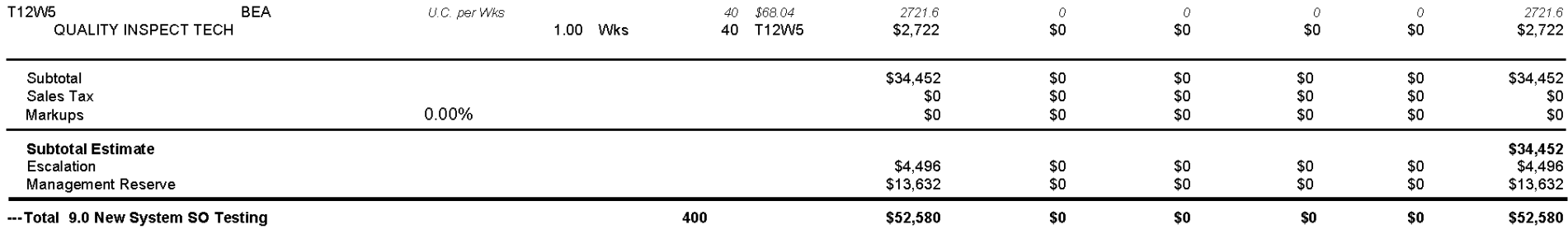

$10.1 \mathrm{MSA}$

remo:

Resources for developing the MSA plan are based on the estimator's judgment and concurred by the project team.

"Develop MSA Plan"* BEA

MANAGER, OPERATIONS ${ }^{\text {BEA }}$

PROJECT ENGINEER BEA

BEA

BEA

${ }^{*}$ Conduct MSA ${ }^{* *}$

(1)

MANAGER, SCIENG FUNCTION

MANAGER, FAC SUPPORT SERVICES

$$
\text { uc. per }
$$

uc. pertirs

$$
\text { UC. per Hrs }
$$$$
\text { u.c. per }
$$

U.c. per Whs

2.00 Wks

2.00 Wks BEA

05/11/2012 11:08:15

Client: C.P.Ischay

Prepared By: A. W. Miller/S. N. Wasley

Estimate Type: Class-5

Equipment Material Subcontractor Other TOTAL

\section{Qty UOM Hrs Resource Labor}

27210

\section{\begin{tabular}{l}
452 \\
$\$ 0$ \\
$\$ 0$ \\
\hline
\end{tabular}} \begin{tabular}{l}
$\$ 34,452$ \\
$\$ 4,496$ \\
$\$ 13632$ \\
\hline
\end{tabular} 52,580

DETAIL ITEM REPORT 


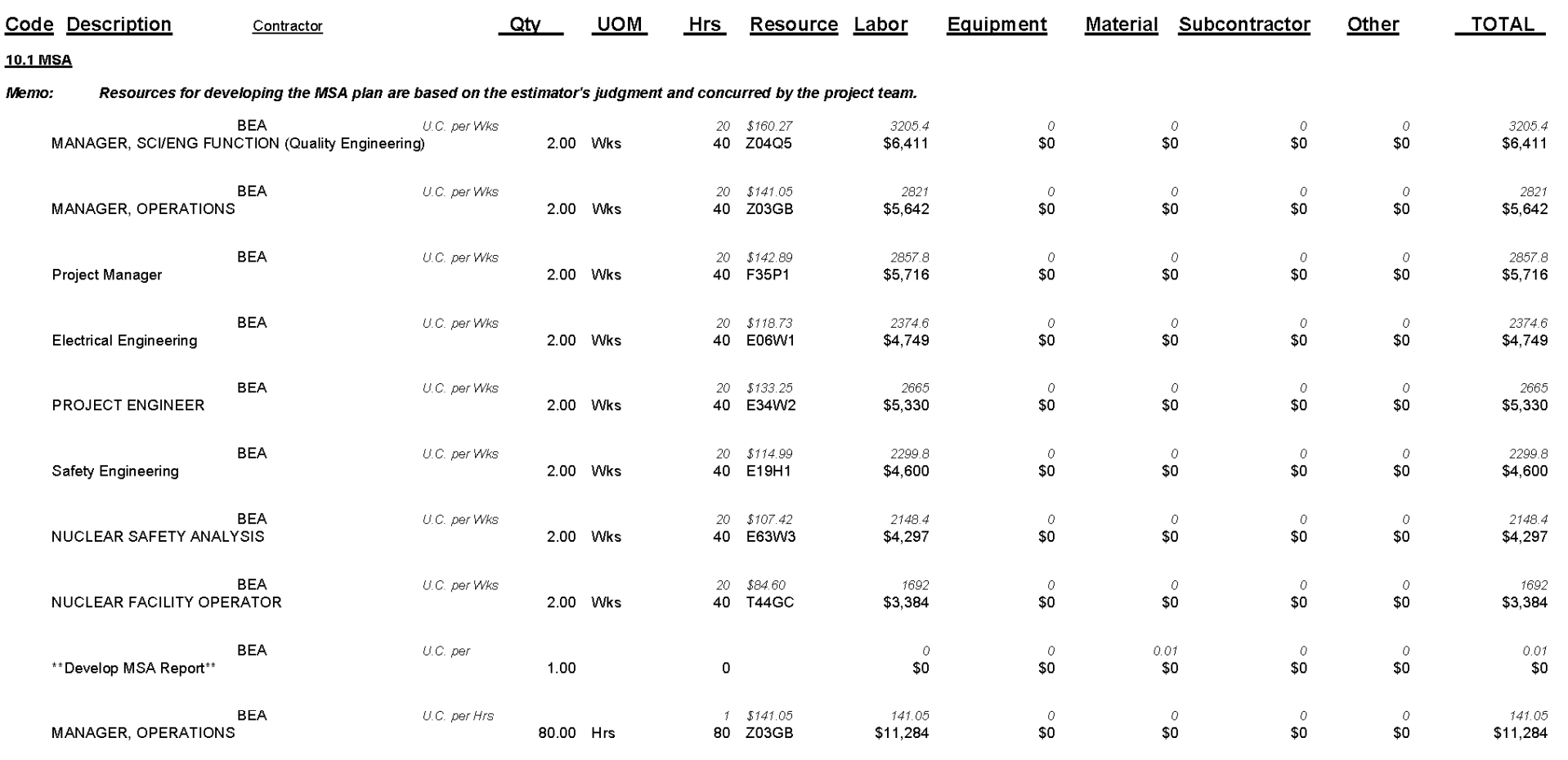

Project Name: RTC Waste Heat Recovery

Project Location: ATR

DETAIL ITEM REPORT

Prepared By: A. W. Miller/S. N. Wasley

Estimate Type: Class-5

$\bar{\infty}$
Client: $\quad$ C.P. Ischay $\begin{array}{rrr}1 & \$ 14 \\ 80 & 2036\end{array}$

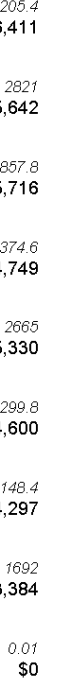

\section{1} $\$ 141,285$ 
Project Name: RTC Waste Heat Recovery

Project Location: ATR

Code Description

Contractor

10.1 MISA

Memo:

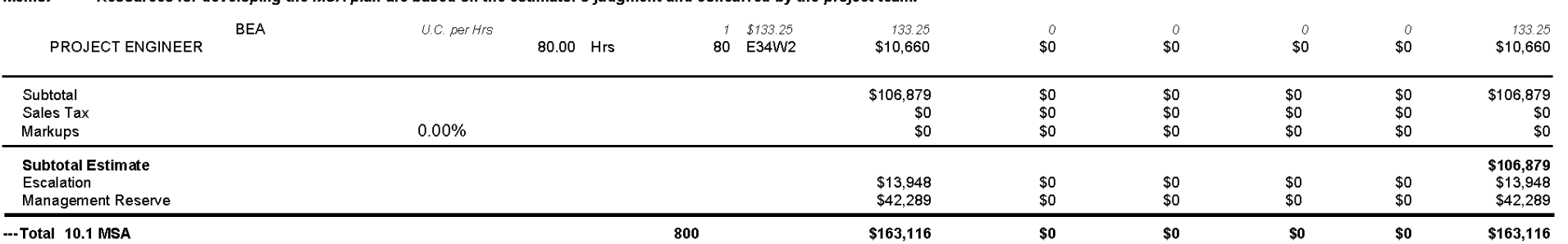

11.1.1 Provide PM Oversight - Level of Effort (LOE) during Transition/Closeout Phase

Memo: Based on estimator judgment including consideration for the duration of the heat recovery system closeout phase.

\begin{tabular}{|c|c|c|c|c|c|c|c|c|c|}
\hline $\begin{array}{l}\text { Provide PM Oversight BEA } \\
\& \text { Cost \& Schedule Monitoring during the Closeout }\end{array}$ & Uc. perwooks & 10.00 weeks & $\begin{array}{rl}10 & \$ 142.89 \\
100 & \mathrm{~F} 35 \mathrm{P} 1\end{array}$ & $\begin{array}{r}1428.9 \\
\$ 14,289\end{array}$ & $\$ 0$ & \$0 & $\$ 0$ & \$o & $\$ 1428.98$ \\
\hline $\begin{array}{l}\text { BEA } \\
\text { Procure Misc. Project Supplies, paper, signage, } \\
\text { office supplies, etc. - Closeout }\end{array}$ & Uc. per iot & $1.00 \begin{array}{c}\text { NM60 } \\
\text { lot }\end{array}$ & 0 & \$o & $\$ 0$ & $\$ 250$ & $\begin{array}{r}0 \\
\$ 0\end{array}$ & $\$ 0$ & $\$ 250$ \\
\hline $\begin{array}{l}\text { Subtotal } \\
\text { Sales Tax } \\
\text { Markups }\end{array}$ & $0.00 \%$ & & & $\begin{array}{r}\$ 14,289 \\
\$ 0 \\
\$ 0\end{array}$ & $\begin{array}{l}\$ 0 \\
\$ 0 \\
\$ 0\end{array}$ & $\begin{array}{r}\$ 250 \\
\$ 15 \\
\$ 0\end{array}$ & $\begin{array}{l}\$ 0 \\
\$ 0 \\
\$ 0\end{array}$ & $\begin{array}{l}\text { \$0 } \\
\text { \$o } \\
\text { \$0 }\end{array}$ & $\begin{array}{r}\$ 14,539 \\
\$ 15 \\
\$ 0\end{array}$ \\
\hline $\begin{array}{l}\text { Subtotal Estimate } \\
\text { Escalation } \\
\text { Management Reserve }\end{array}$ & & & & $\begin{array}{l}\$ 1,865 \\
\$ 5,654\end{array}$ & $\begin{array}{l}\$ 0 \\
\$ 0\end{array}$ & $\begin{array}{r}\$ 35 \\
\$ 105\end{array}$ & $\begin{array}{l}\$ 0 \\
\$ 0\end{array}$ & $\begin{array}{l}\$ 0 \\
\$ 0\end{array}$ & $\begin{array}{l}\$ 1,454 \\
\$ 1,899 \\
\$ 5,759\end{array}$ \\
\hline $\begin{array}{l}\text { otal 11.1.1 Provide PM Oversight - Level of Effo } \\
\text { during Transition/Closeout Phase }\end{array}$ & & & & $\$ 21,808$ & \$o & $\$ 404$ & $\$ 0$ & \$o & \\
\hline
\end{tabular}


Project Name: RTC Waste Heat Recovery

Project Location: ATR

Code Description

Contractor

11.1.2 Closeout Prolect Flles

Memo:

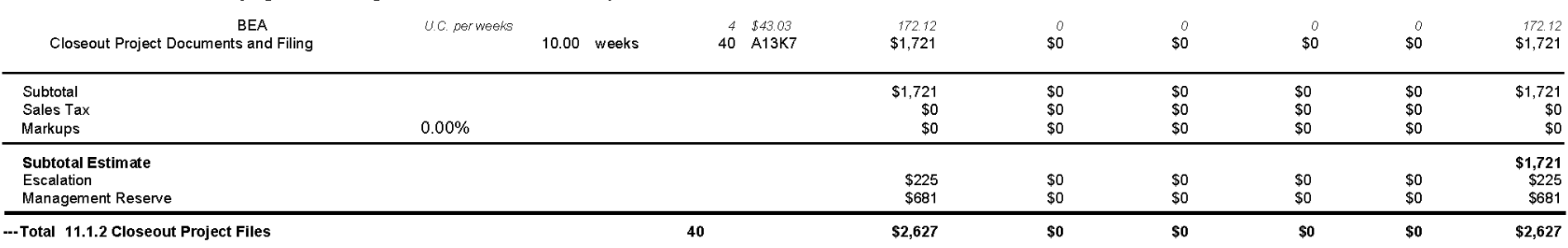

\section{DETAIL ITEM REPORT}

lient: C.P.Ischay

Prepared By: A.W.Miller/S. N. Wasley

Estimate Type: Class-5

rial Subcontractor Other TOTAL
40

11.2.1 Prepare Master Facility As-Built Drawings and Incorporate into EDMS

Memo: Estimator judgment and concurrence with the project team.

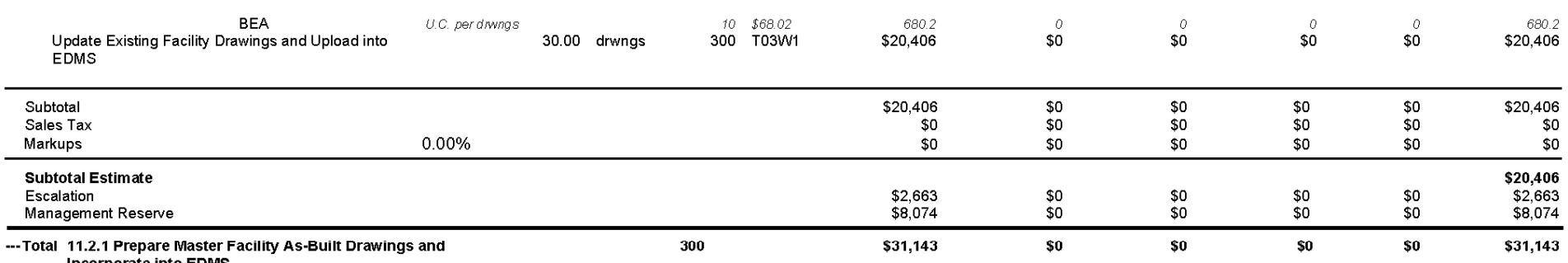

11.2.1 Prepare Master F
Incorporate into EDMS 
Project Name: RTC Waste Heat Recovery

Project Location: ATR

\section{Code Description Contractor}

11.2.2 Prepare Prolect As-Bullt Drawings and Incoporate into ED

Memo: Estimator judgment and concurrence with the project tea

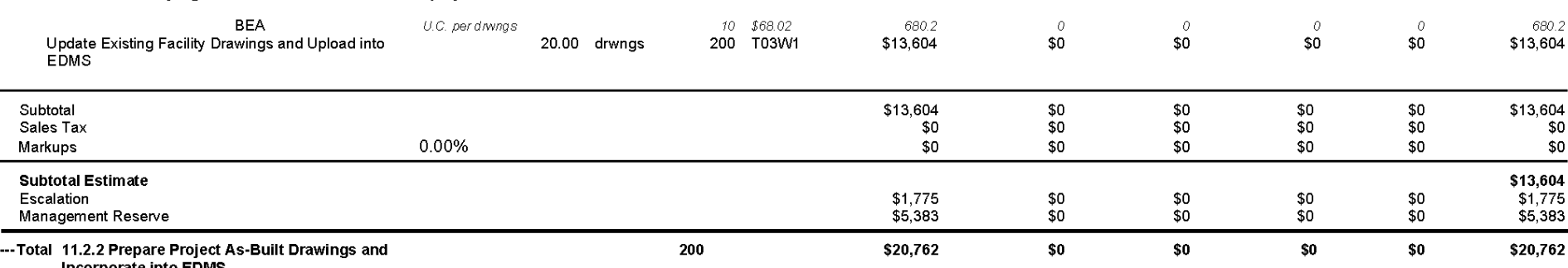

DETAIL ITEM REPORT

Client: C.P.Ischay

Prepared By: A. W. Miller/S. N. Wasley
Estimate Type: Class-5

Subcontractor Other TOTAL
Incorporate into EDMS

\subsubsection{Complete Closeout PM Checklist}

Memo: Estimator judgment and concurrence with the project team.

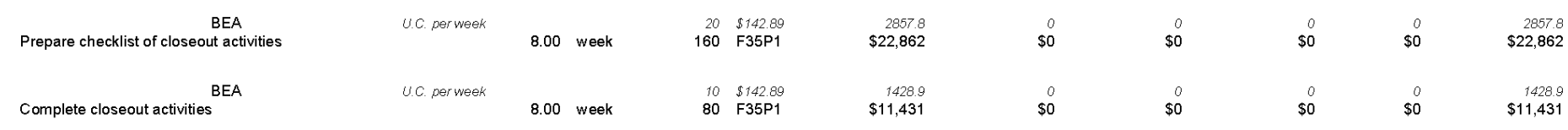

BEA

11:08:15 
Project Name: RTC Waste Heat Recovery

Project Location: ATR

Estimate Number: 7850

Code Description $\quad$ contractor 11.3.1.1 Complete Closeout PM CneckIIst

Memo: Estimator judgment and concurrence with the project team.

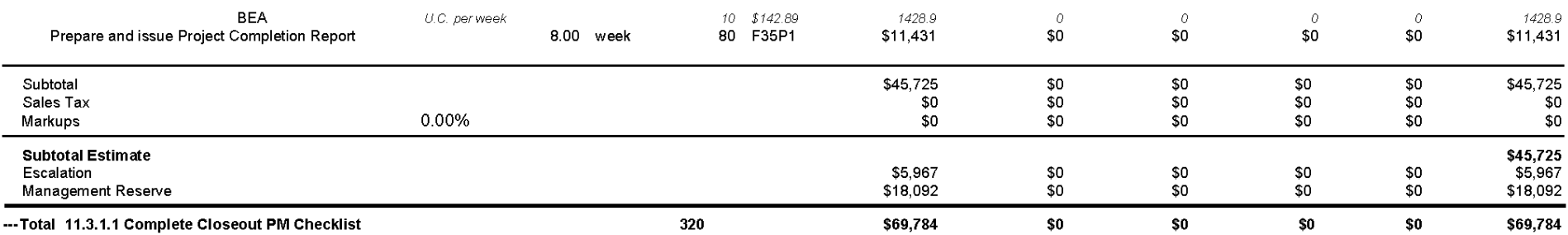

\subsubsection{Develop Lessons Learned}

Memo: Estimator judgment and concurrence with the project team.

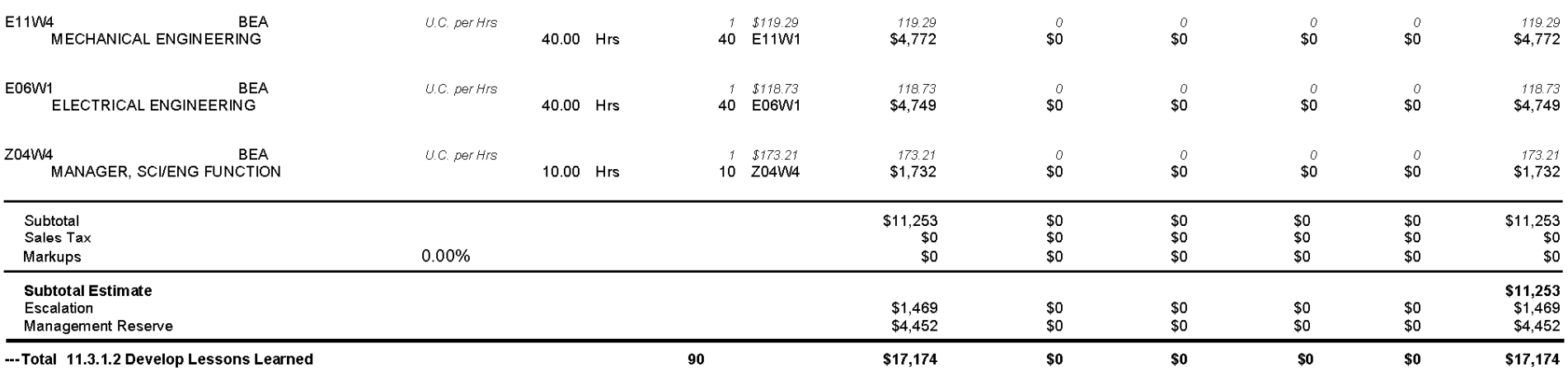

BEA

5/11/2012 11:08:15
DETAIL ITEM REPORT

Client: $\quad$ C. P. Ischay

Prepared By: A. W. Miller/S. N. Wasley

\section{Qty UOM Hrs Resource Labor Equipment Material Subcontractor Other TOTAL}

Material costs where applicable include Idaho State Sales Tax Page No. $\quad 102$ 
Project Name: RTC Waste Heat Recovery

Project Location: ATR

Estimate Number: 7850

Code Description contractor

11.3.1.3 Prepare Final Prolect Closeout Repont

Memo: Estimator judgmert and concurrencewith the poject

\begin{tabular}{|c|c|c|c|c|c|c|c|c|c|}
\hline $\begin{array}{l}\text { F35P1 } \\
\text { PROJECT MANAGER }\end{array}$ & Uc. perthrs & $40.00 \mathrm{Hrs}$ & $\begin{array}{rl}1 & \$ 142.89 \\
40 & \mathrm{~F} 35 \mathrm{P} 1\end{array}$ & $\begin{array}{r}142.89 \\
\$ 5,716\end{array}$ & $\begin{array}{l}0 \\
\$ 0\end{array}$ & \$o & \$o & \$o & $\begin{array}{r}142.89 \\
\$ 5,716\end{array}$ \\
\hline $\begin{array}{l}\text { Subtotal } \\
\text { Sales Tax } \\
\text { Markups }\end{array}$ & $0.00 \%$ & & & $\begin{array}{r}\$ 5,716 \\
\$ 0 \\
\$ 0\end{array}$ & $\begin{array}{l}\$ 0 \\
\$ 0 \\
\$ 0\end{array}$ & $\begin{array}{l}\$ 0 \\
\$ 0 \\
\$ 0\end{array}$ & $\begin{array}{l}\$ 0 \\
\$ 0 \\
\$ 0\end{array}$ & $\begin{array}{l}\$ 0 \\
\$ 0 \\
\$ 0\end{array}$ & $\begin{array}{r}\$ 5,716 \\
\$ 0 \\
\$ 0\end{array}$ \\
\hline $\begin{array}{l}\text { Subtotal Estimate } \\
\text { Escalation } \\
\text { Management Reserve }\end{array}$ & & & & $\begin{array}{r}\$ 746 \\
\$ 2,262\end{array}$ & $\begin{array}{l}\$ 0 \\
\$ 0\end{array}$ & $\begin{array}{l}\$ 0 \\
\$ 0\end{array}$ & $\begin{array}{l}\$ 0 \\
\$ 0\end{array}$ & $\begin{array}{l}\$ 0 \\
\$ 0\end{array}$ & $\begin{array}{r}\$ 5,716 \\
\$ 746 \\
\$ 2,262\end{array}$ \\
\hline Total 11.3.1.3 Prepare Fi & port & & & $\$ 8,723$ & $\$ 0$ & so & so & \$o & $\$ 8,723$ \\
\hline
\end{tabular}

$\bar{\omega}$

\subsubsection{Provide Operational Training}

Memo: Estimator judgment and concurence with the project team.

Develop Training Plan \& Written Test

BEA

U.C. per Allow

1.00

$\begin{array}{ll}40 & \$ 82.61 \\ 40 & \text { P37Y5 }\end{array}$

3304.4
$\$ 3,304$

$\begin{array}{ll}45 & 58261 \\ 45 & \text { P37Y5 }\end{array}$

3717,45
$\$ 3,717$

1.00 Allow

U.C. perfTE

4.00 FTE

$\begin{array}{ll}15 & \$ 67.97 \\ 60 & \text { U11GB }\end{array}$

$\begin{array}{rr}15 & \$ 100.78 \\ 120 & \text { F05GB }\end{array}$

$\$ 019.55$

$\$ 13,174$
UC por ETE

0.C. portte $8.00 \mathrm{FTE}$

BEA
Client: C.P.Ischay

Prepared By: A. W. Miller/S. N. Wasley
Estimate Type: Class-5

Equipment Material Subcontractor Other TOTAL

\begin{tabular}{|c|c|c|c|c|c|c|c|c|c|c|c|c|}
\hline $\mathrm{U}_{\text {ELECTRICIAN }}$ & BEA & U.C. perfte & 4.00 & FTE & $\begin{array}{l}15 \\
60\end{array}$ & $\begin{array}{l}567.97 \\
\text { U11GB }\end{array}$ & $\begin{array}{l}1019.55 \\
\$ 4,078\end{array}$ & $\begin{array}{l}0 \\
\$ 0\end{array}$ & $\begin{array}{c}0 \\
\$ 0\end{array}$ & $\begin{array}{r}0 \\
\$ 0\end{array}$ & $\$ 0$ & $\begin{array}{l}1079,55 \\
\$ 4,078\end{array}$ \\
\hline
\end{tabular}

BEA

5/11/2012 11:08:15 
Project Name: RTC Waste Heat Recovery

Project Location: ATR

Code Description contractor

11.4.1 Provlde Operational Training

Memo: Estimator judgment and concurrence with the project team.

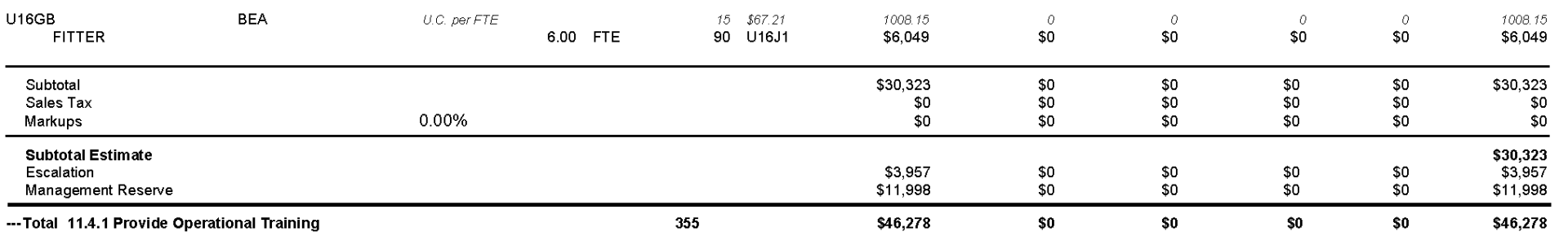

11.4.2 Provide Start-Up Coordination, Materials, \& Supplies

Memo: Estimator judgment and concurence with the project team.

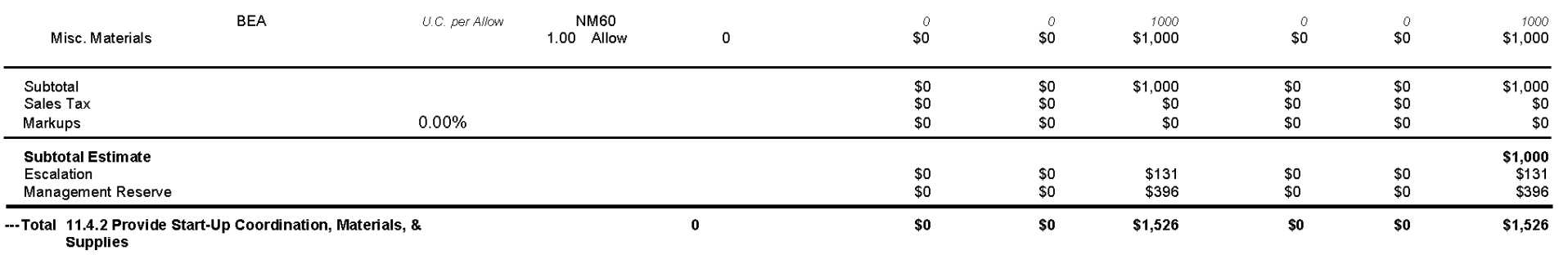


Project Name: RTC Waste Heat Recovery

Project Location: ATR

Pstimate Number: 7850

Code Description

11.4.3 Provide Spares

\section{Memo: Estimator judgment and concurrence with the project team}

BEA U. per Allow

Spare Parts Allowance for New Heat Recovery System . per A/low $1.00 \mathrm{NM60}$

nce only for spare parts.

Incorporate New Spares into Warehouse

U.C. per Allow

1.00 Allow

Subtotal
Sales Tax

Sales Tax
Markups

Subtotal Estimate

Escalation
Management Reserve

---Total 11.4.3 Provide Spares

DETAIL ITEM REPORT

lient: C. P. Ischay

Prepared By: A. W. Miller/S. N. Wasley

Estimate Type: Class-5

\section{aty UOM Hrs Resource Labor Equipment Material Subcontractor other TOTAL}

12.1 System Maintenance (5 Years)

Memo: Estimated allowance is based on approximately $2 \%$ per year of the initial system instaflation costs. This allowance is to maimain the heat recovery system including system cleaning for corrosion, glycol solution changeout, and equipment repairs

\begin{tabular}{|c|c|c|c|c|c|c|c|c|c|c|}
\hline $\begin{array}{c}\text { BEA } \\
\text { Heat Recovery System Maintenance (Year 1) }\end{array}$ & UC. per Allow & 1.00 & Allow & $\begin{array}{r}300 \\
300\end{array}$ & $\begin{array}{l}\$ 68.47 \\
\mathrm{U} 29 \mathrm{~GB}\end{array}$ & $\begin{array}{r}20541 \\
\$ 20,541\end{array}$ & $\begin{aligned} 0 \\
\$ 0\end{aligned}$ & $\begin{array}{r}50000 \\
\$ 50,000\end{array}$ & $\begin{array}{r}0 \\
\$ 0\end{array}$ & $\$ 0$ \\
\hline $\begin{array}{l}\text { BEA } \\
\text { Heat Recovery System Maintenance (Year 2) }\end{array}$ & U.C. per Allow & 1.00 & Allow & $\begin{array}{r}300 \\
300\end{array}$ & $\begin{array}{l}\$ 68.47 \\
\text { U29GB }\end{array}$ & $\begin{array}{r}20541 \\
\$ 20,541\end{array}$ & $\stackrel{0}{\$ 0}$ & $\begin{array}{r}50000 \\
\$ 50,000\end{array}$ & $\begin{array}{r}0 \\
\$ 0\end{array}$ & $\$ 0$ \\
\hline $\begin{array}{c}\text { BEA } \\
\text { Heat Recovery System Maintenance (Year 3) }\end{array}$ & UC. per Allow & 1.00 & Allow & $\begin{array}{r}300 \\
300\end{array}$ & $\begin{array}{l}\$ 68.47 \\
\cup 29 \mathrm{~GB}\end{array}$ & $\begin{array}{r}20541 \\
\$ 20,541\end{array}$ & $\$ 0$ & $\begin{array}{r}50000 \\
\$ 50,000\end{array}$ & $\begin{array}{r}0 \\
\$ 0\end{array}$ & $\$ 0$ \\
\hline Heat Recovery System Maintenance (Year 4) & UC. per Allow & 1.00 & Allow & $\begin{array}{r}300 \\
300\end{array}$ & $\begin{array}{l}\$ 68.47 \\
\cup 29 G B\end{array}$ & $\begin{array}{r}20541 \\
\$ 20,541\end{array}$ & $\$ 0$ & $\begin{array}{r}50000 \\
\$ 50,000\end{array}$ & $\begin{array}{r}0 \\
\$ 0\end{array}$ & \$o \\
\hline
\end{tabular}


Project Name: RTC Waste Heat Recovery

Project Location: ATR

Pstimate Number:7850

Code Description

Code Description

Intenance (5 Years)

Estimated allowance is based on approximately $2 \%$ per year of

\begin{tabular}{|c|c|c|c|c|c|c|c|c|c|}
\hline Heat Recovery System Maintenance (Year 5) & U.C. per Allow & 1.00 Allow & $\begin{array}{ll}300 & \$ 68.47 \\
300 & \mathrm{U} 29 \mathrm{~GB}\end{array}$ & $\begin{array}{r}20547 \\
\$ 20,541\end{array}$ & \$0 & $\begin{array}{r}50000 \\
\$ 50,000\end{array}$ & $\begin{array}{l}0 \\
\$ 0\end{array}$ & $\begin{array}{l}0 \\
\$ 0\end{array}$ & $\begin{array}{r}70541 \\
\$ 70,541\end{array}$ \\
\hline $\begin{array}{l}\text { Subtotal } \\
\text { Sales Tax }\end{array}$ & & & & $\begin{array}{r}\$ 102,705 \\
\$ 0\end{array}$ & $\begin{array}{l}\$ 0 \\
\$ 0\end{array}$ & $\begin{array}{r}\$ 250,000 \\
\$ 15,000\end{array}$ & $\begin{array}{l}\$ 0 \\
\$ 0\end{array}$ & $\begin{array}{l}\$ 0 \\
\$ 0\end{array}$ & $\begin{array}{r}\$ 352,705 \\
\$ 15,000\end{array}$ \\
\hline Markups & $0.00 \%$ & & & $\$ 0$ & $\$ 0$ & $\$ 0$ & $\$ 0$ & $\$ 0$ & $\$ 0$ \\
\hline Total 12.1 System Maintenanc & & & & $\$ 156,746$ & \$o & $\$ 404,436$ & so & $\$ 0$ & $\$ 561,182$ \\
\hline
\end{tabular}

\subsection{System Operating Costs (5 Years)}

Memo: Estimated allowance is based on estimator's judgment of the approximate number of identified yearly reactor outages when the system would be shut down and started back up. The identified unit hours would cover system operators, procedure records, and report development.

\begin{tabular}{|c|c|c|c|c|c|c|c|c|c|c|c|}
\hline $\begin{array}{l}\text { Hea } \\
\text { 1) }\end{array}$ & U.c. per Shut Dwn & 6.00 & Shut Dwn & $\begin{array}{r}20 \\
120\end{array}$ & $\begin{array}{l}\$ 68.47 \\
\cup 29 G B\end{array}$ & $\begin{array}{r}1369.4 \\
\$ 8,216\end{array}$ & $\$ 0$ & \$0 & $\begin{array}{l}0 \\
\$ 0\end{array}$ & $\$ 0$ & $\begin{array}{r}\$ 369.4 \\
\$ 8,216\end{array}$ \\
\hline $\begin{array}{l}\text { Hea } \\
\text { Heat System Shut Down for Reactor Shut Down (rear }\end{array}$ & uc. per shut own & 6.00 & Shut Dwn & $\begin{array}{r}20 \\
120\end{array}$ & $\begin{array}{l}\$ 68.47 \\
\cup 296 \mathrm{~B}\end{array}$ & $\begin{array}{r}1369.4 \\
\$ 8,216\end{array}$ & $\$ 0$ & $\$ 0$ & $\$ 0$ & $\$ 0$ & $\begin{array}{r}1369.4 \\
\$ 8,216\end{array}$ \\
\hline $\begin{array}{l}\text { BEA } \\
\text { Heat System Shut Down for Reactor Shut Down (Year } \\
\text { 3) }\end{array}$ & U.c. per Shut Dwn & 6.00 & Shut Dwח & $\begin{array}{r}20 \\
120\end{array}$ & $\begin{array}{l}\$ 68.47 \\
\cup 296 \mathrm{~B}\end{array}$ & $\begin{array}{r}1369.4 \\
\$ 8,216\end{array}$ & $\begin{array}{r}0 \\
\$ 0\end{array}$ & $\begin{array}{l}0 \\
\$ 0\end{array}$ & $\begin{array}{r}0 \\
\text { \$o }\end{array}$ & \$0 & $\begin{array}{r}1369.4 \\
\$ 8,216\end{array}$ \\
\hline $\begin{array}{l}\text { BEA } \\
\text { Heat System Shut Down for Reactor Shut Down (Year } \\
\text { 4) }\end{array}$ & 40. per Shut Dwn & 6.00 & Shut Dwn & $\begin{array}{r}20 \\
120\end{array}$ & $\begin{array}{l}\$ 68.47 \\
\cup 296 \mathrm{~B}\end{array}$ & $\begin{array}{r}1369.4 \\
\$ 8,216\end{array}$ & $\$ 0$ & $\$ 0$ & $\begin{array}{l}0 \\
\$ 0\end{array}$ & \$o & $\begin{array}{r}1369.4 \\
\$ 8,216\end{array}$ \\
\hline $\begin{array}{l}\text { BEA } \\
\text { Heat System Shut Down for Reactor Shut Down (Year } \\
\text { 5) }\end{array}$ & U.c. per Shut Dwn & 6.00 & Shut Dwn & $\begin{array}{r}20 \\
120\end{array}$ & $\begin{array}{l}\$ 60.47 \\
\text { U29GB }\end{array}$ & $\begin{array}{r}1369.4 \\
\$ 8,216\end{array}$ & $\$$ & $\$ 0$ & $\begin{array}{l}0 \\
\$ 0\end{array}$ & $\$ 0$ & $\begin{array}{r}1369.4 \\
\$ 8,216\end{array}$ \\
\hline $11: 08: 15$ & & & & & $\cos t E S$ & & & wh & incl & 0. & \\
\hline
\end{tabular}

DETAIL ITEM REPORT

Client: C.P.Ischay

Prepared By: A.W.Miller/S. N. Wasley

\section{Qty UOM Hrs Resource Labor Equipment Material Subcontractor Other TOTAL}

stimate Type: Class-5

61,182 
Project Name: RTC Waste Heat Recovery

Project Location: ATR

Code Description Contractor

12.2 Svstem operatina cosis 15 rears

Memo:

stimated allowance is based on estimator's judgment of the approximate number of identified yearly reactor outages when the system would be shut down and started back up. The

\begin{tabular}{|c|c|c|c|c|c|c|c|c|c|c|c|}
\hline $\begin{array}{l}\text { BEA } \\
\text { Heat System Start-up for Reactor Start-up (Year 1) }\end{array}$ & u.c. per shut own & 6.00 & Shut Dwn & $\begin{array}{r}20 \\
120\end{array}$ & $\begin{array}{l}\frac{968.47}{U} \\
\cup 296 \mathrm{~B}\end{array}$ & $\begin{array}{r}1369.4 \\
\$ 8,216\end{array}$ & $\$$ & $\begin{aligned} 0 \\
\$ 0\end{aligned}$ & $\begin{array}{l}0 \\
\$ 0\end{array}$ & $\$ 0$ & $\begin{array}{r}1369,4 \\
\$ 8,216\end{array}$ \\
\hline $\begin{array}{c}\text { BEA } \\
\text { Heat System Start-up for Reactor Start-up (Year 2) }\end{array}$ & U.C. per Shut Dwn & 6.00 & Shut Dwn & ${ }_{120}^{20}$ & $\begin{array}{l}\$ 68.47 \\
\text { U29GB }\end{array}$ & $\begin{array}{r}1369.4 \\
\$ 8,216\end{array}$ & $\$ 0$ & $\$ 0$ & $\$ 0$ & $\$ 0$ & $\$ 8,216$ \\
\hline Heat System Start-up for Reactor Start-up (Year 3) & U.C. per Shut Dwn & 6.00 & Shut Dwn & $\begin{array}{r}200 \\
120\end{array}$ & $\begin{array}{l}\$ 68.47 \\
\mathrm{U} 29 \mathrm{~GB}\end{array}$ & $\begin{array}{r}1369.4 \\
\$ 8,216\end{array}$ & \$o & \$o & $\$ 0$ & $\$ 0$ & $\$ 8,216$ \\
\hline $\begin{array}{c}\text { BEA } \\
\text { Heat System Start-up for Reactor Start-up (Year 4) }\end{array}$ & U.C per Shut Dwn & 6.00 & Shut Dwn & 120 & $\begin{array}{l}560.47 \\
\mathrm{U} 29 \mathrm{~GB}\end{array}$ & $\begin{array}{r}1389.4 \\
\$ 8,216\end{array}$ & \$o & $\$ 0$ & $\$ 0$ & $\$ 0$ & $\$ 8,216$ \\
\hline $\begin{array}{l}\text { BEA } \\
\text { Heat System Start-up for Reactor Start-up (Year 5) }\end{array}$ & U.C. per Shut Dwn & 6.00 & Shut Dwn & 120 & 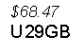 & $\begin{array}{l}1369.4 \\
\$ 8,216\end{array}$ & \$0 & $\$ 0$ & $\$ 0$ & $\$ 0$ & $\$ 8,216$ \\
\hline $\begin{array}{l}\text { total } \\
\text { s Tax } \\
\text { sups }\end{array}$ & $0.00 \%$ & & & & & $\begin{array}{r}\$ 82,164 \\
\$ 0 \\
\$ 0\end{array}$ & $\begin{array}{l}\$ 0 \\
\$ 0 \\
\$ 0\end{array}$ & $\begin{array}{l}\$ 0 \\
\$ 0 \\
\$ 0\end{array}$ & $\begin{array}{l}\$ 0 \\
\$ 0 \\
\$ 0\end{array}$ & $\begin{array}{l}\$ 0 \\
\$ 0 \\
\$ 0\end{array}$ & $\begin{array}{r}\$ 82,164 \\
\$ 0 \\
\$ 0\end{array}$ \\
\hline $\begin{array}{l}\text { total Estimate } \\
\text { alation } \\
\text { lagement Reserve }\end{array}$ & & & & & & $\begin{array}{l}\$ 10,722 \\
\$ 32,510\end{array}$ & 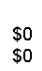 & $\begin{array}{l}\$ 0 \\
\$ 0\end{array}$ & $\begin{array}{l}\$ 0 \\
\$ 0\end{array}$ & $\$ 0$ & $\begin{array}{l}\$ 82,164 \\
\$ 10,722 \\
\$ 32,510\end{array}$ \\
\hline 12.2 System Operating costs (5 & & & & & & $\$ 125,397$ & so & so & so & \$0 & $\$ 125,397$ \\
\hline
\end{tabular}

\begin{tabular}{|c|c|c|c|c|c|c|}
\hline $\begin{array}{l}\text { Subtotal RTC Waste Heat Recovery - INDIRECT } \\
\text { Sales Tax } \\
\text { Markups }\end{array}$ & $\begin{array}{r}\$ 3,828,740 \\
\$ 0 \\
\$ 327,725\end{array}$ & $\begin{array}{r}\$ 174,671 \\
\$ 0 \\
\$ 81,023\end{array}$ & $\begin{array}{r}\$ 1,036,951 \\
\$ 58,859 \\
\$ 379,734\end{array}$ & $\begin{array}{r}\$ 448,739 \\
\$ 0 \\
\$ 210,878\end{array}$ & $\begin{array}{l}\$ 0 \\
\$ 0 \\
\$ 0\end{array}$ & $\begin{array}{r}\$ 5,489,101 \\
\$ 58,859 \\
\$ 999,361\end{array}$ \\
\hline $\begin{array}{l}\text { Subtotal Estimate } \\
\text { Escalation } \\
\text { Management Reserve }\end{array}$ & $\begin{array}{r}\$ 389,813 \\
\$ 1,591,197\end{array}$ & $\begin{array}{l}\$ 23,703 \\
\$ 97,789 \\
\end{array}$ & $\begin{array}{l}\$ 148,794 \\
\$ 568,518\end{array}$ & $\begin{array}{r}\$ 61,146 \\
\$ 252,267\end{array}$ & $\begin{array}{l}\$ 0 \\
\$ 0\end{array}$ & $\begin{array}{r}\$ 6,547,320 \\
\$ 623,456 \\
\$ 2,509,772 \\
\end{array}$ \\
\hline Total RTC Waste Heat Recovery - INDIRECT & $\$ 6,137,475$ & $\$ 377,186$ & $\$ 2,192,856$ & $\$ 973,030$ & $\$ 0$ & $\$ 9,680,547$ \\
\hline
\end{tabular}

11:08:1

\section{DETAIL ITEM REPORT}

Client: C.P.Ischay

Prepared By. A.W.Miler/S. N. Wasley

\section{Qty UOM Hrs Resource Labor Equipment Material Subcontractor Other TOTAL}

216 216 216 